\title{
DESIGN, IMPLEMENTATION, AND TEST OF A MICRO FORCE DISPLACEMENT SYSTEM
}

\author{
A Thesis \\ presented to \\ the Faculty of California Polytechnic State University, \\ San Luis Obispo
}

\author{
In Partial Fulfillment \\ of the Requirements for the Degree \\ Master of Science in Engineering
}

by

Evan Derek Cate

June 2014 
(C) 2014

Evan Derek Cate

ALL RIGHTS RESERVED 
TITLE:

AUTHOR:

DATE SUBMITTED:

COMMITTEE CHAIR:

COMMITTEE MEMBER:

COMMITTEE MEMBER:
Design, Implementation, and Test of a Micro Force Displacement System

Evan Derek Cate

June 2014

Richard Savage, $\mathrm{PhD}$

Professor of Biomedical Engineering

David Clague, $\mathrm{PhD}$

Associate Professor of Biomedical Engineering

Linda Vanasupa, $\mathrm{PhD}$

Professor of Materials Engineering 


\begin{abstract}
Design, Implementation, and Test of a Micro Force Displacement System
\end{abstract}

Evan Derek Cate

The design and implementation of a micro-force displacement system was completed to test the force-displacement characteristics of square silicon diaphragms with side lengths of $4 \mathrm{~mm}, 5 \mathrm{~mm}$, and $7 \mathrm{~mm}$ with a thickness of $10 \mathrm{um}$. The system utilizes a World Precision Instruments Fort $10 \mathrm{~g}$ force transducer attached to a World Precession Instruments TBM4M amplifier. A Keithley 2400 source meter provided data acquisition of the force component of the system. A micro prober tip was utilized as the testing probe attached to the force transducer with a tip radius of 5um. The displacement of samples was measured using a Newport M433 linear stage driven by a Newport ESP300 motion controller (force readings at constant displacement intervals). An additional 3 linear stages were used to provide $X$ and $Y$-axis positioning of samples beneath the probe tip. The system components were mounted to an optical bench to provide stability during testing. C\# was used to deliver the code to the individual components of the system. In addition the software provides a graphic user interface for future users that includes a calibration utility (both $X / Y$ and force calibration), live force-displacement graph, motion control, and a live video feed for sample alignment. Calibration of the force transducer was accomplished using an Adam Equipment PGW153e precision balance to assign force values to the voltage data produced from the transducer. Displacement calibration involved the use of a microscope calibration micrometer. The system was characterized with an equipment variability of $\pm 1.02 \mathrm{mg}$ at $1.75 \mathrm{um}$, and $\pm 1.86 \mathrm{mg}$ at $3.5 \mathrm{um}$ with the ability to characterize samples with stiffness less than $279 \mathrm{mg} / \mathrm{um}$. The displacement resolution of the system was determined to be $35 \mathrm{~nm}$ per step of the linear stages. The diaphragms created to test the machine were fabricated from 10um thick device layer SOI wafers. An etch consisting of $38 \mathrm{~g} / \mathrm{l}$ silicic acid, $7 \mathrm{~g} / \mathrm{l}$ ammonium persulfate, and $5 \%$ TMAH was used to reduce the formation of hillocks, and provide a consistent etch rate. A Gage R\&R study was performed on the fabricated diaphragms, indicating that the deflection produced by the $4 \mathrm{~mm}, 5 \mathrm{~mm}$, and $7 \mathrm{~mm}$ diaphragms was resolvable by the machine. A model was developed to correlate theoretical results to the observed measured values.

Keywords: AFM, Micro, Force, Displacement, System, MEMS, Diaphragm, Cantilever, Transducer, SOI 


\section{ACKNOWLEDGMENTS}

I would like to thank my advisor, Dr. Richard Savage for the opportunity to work on this project, his support, advice, and help obtaining funding, Dr. Gary Hughes for his guidance and knowledge in the world of equipment testing and statistics, Kevin Schapansky for his expertise in the software portion of this project. Finally, I would like to thank my family for their financial and emotional support through my college career. 


\section{TABLE OF CONTENTS}

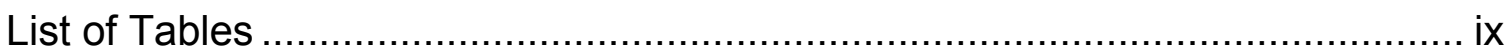

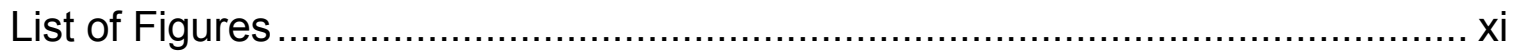

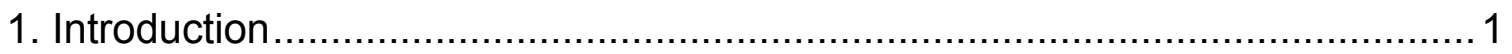

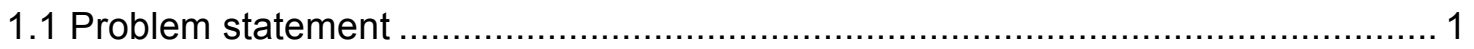

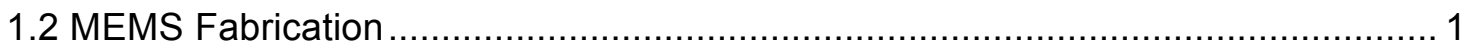

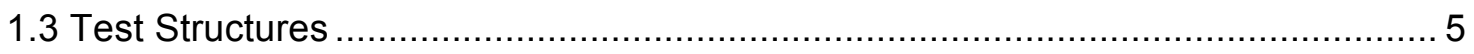

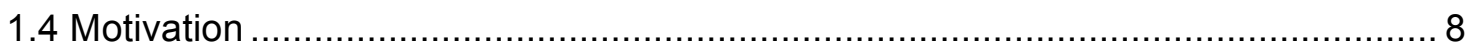

1.5 Force Displacement Systems Currently Available ……………..................... 9

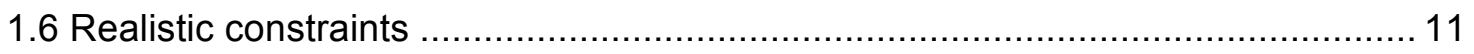

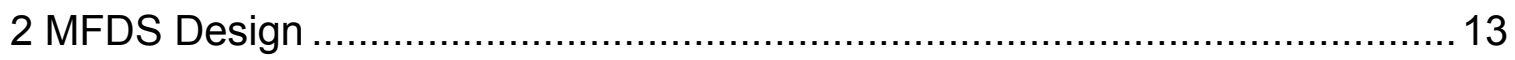

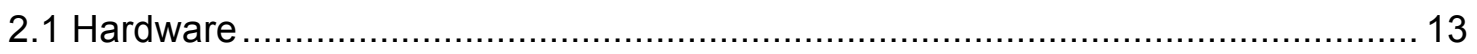

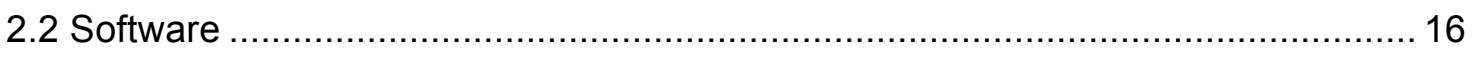

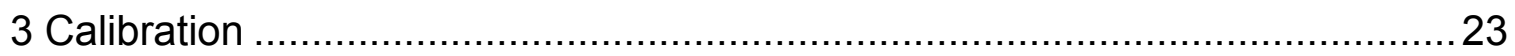

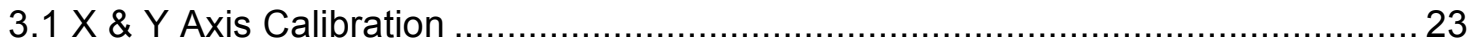

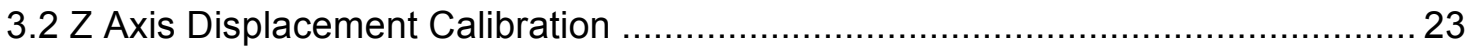

3.3 Systemic Displacement Characterization ........................................................ 24

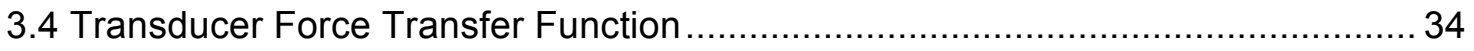

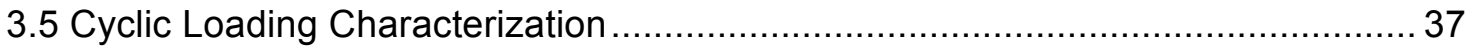

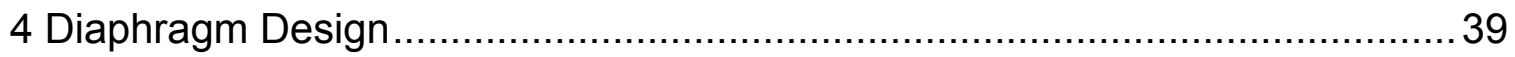

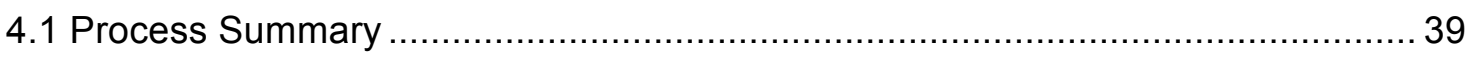

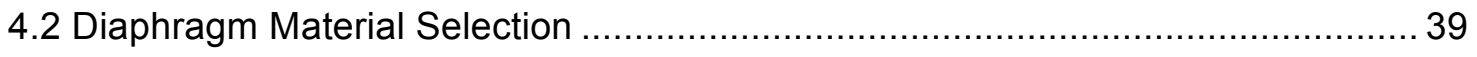

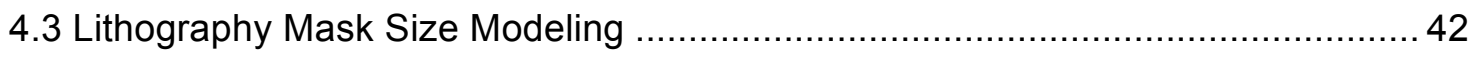




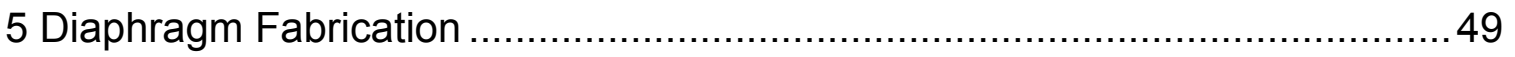

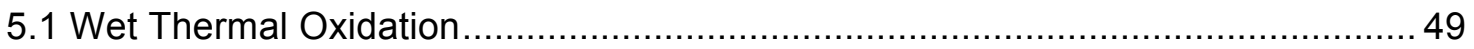

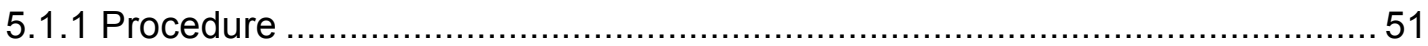

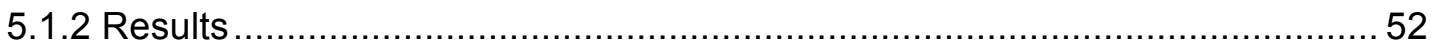

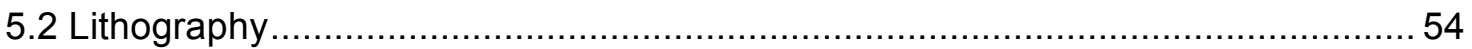

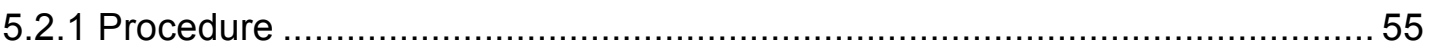

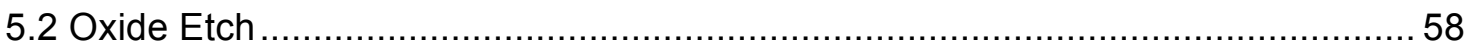

5.2.1 BOE Etch Rate Determination ............................................................... 59

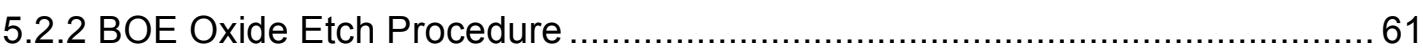

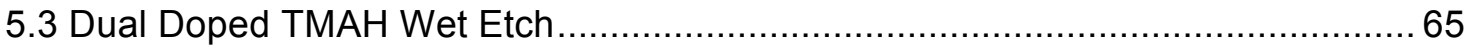

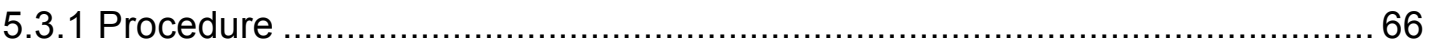

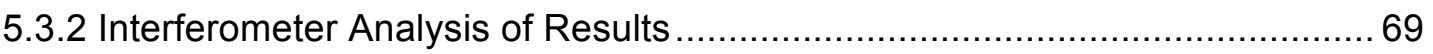

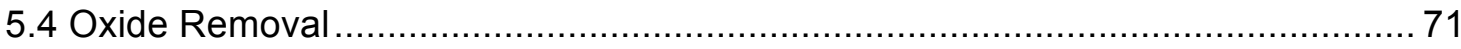

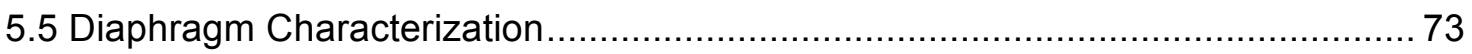

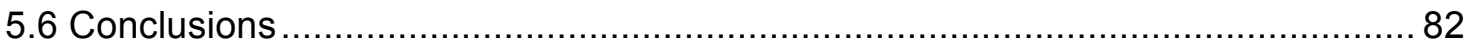

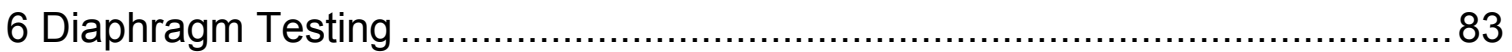

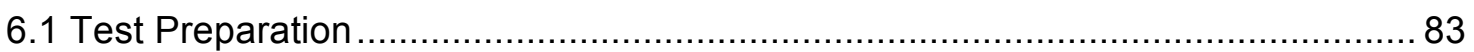

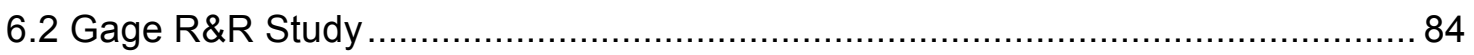

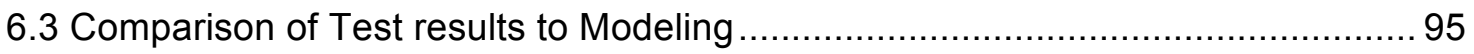

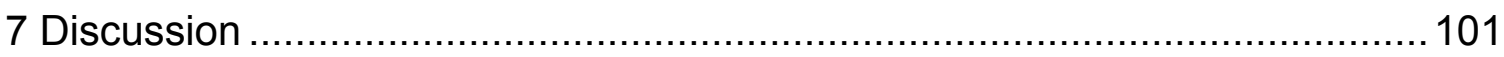

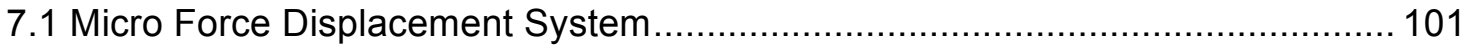

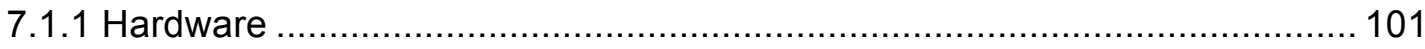

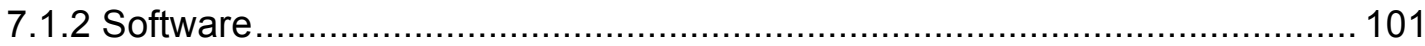

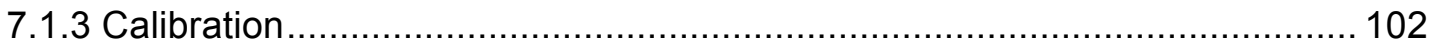

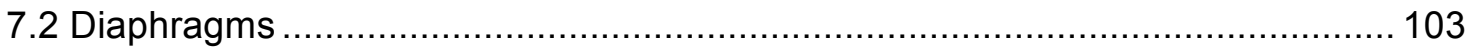




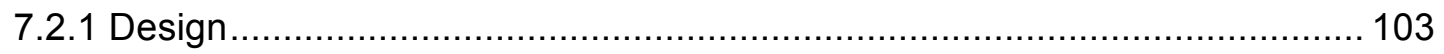

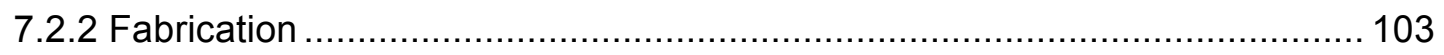

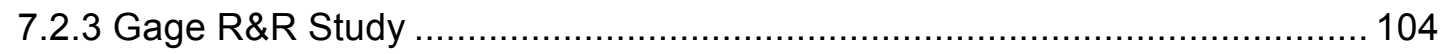

7.2.4 Comparison of Test Results to Modeling ............................................... 105

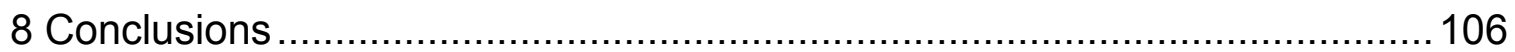

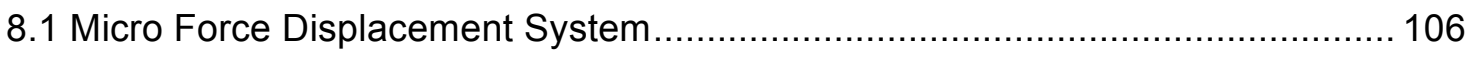

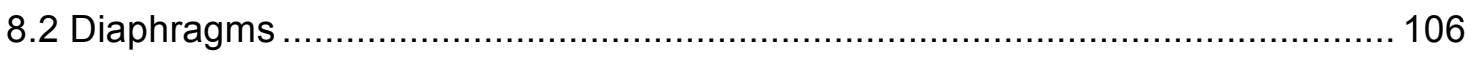

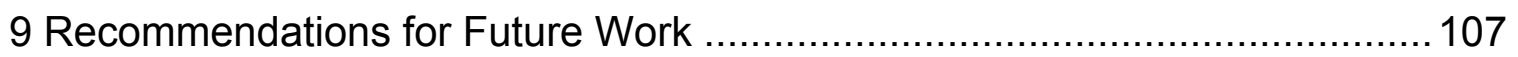

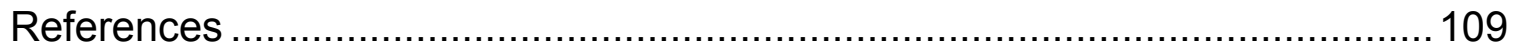

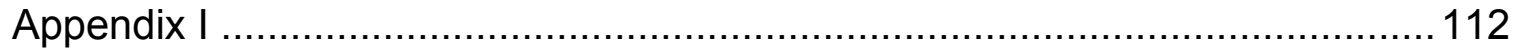

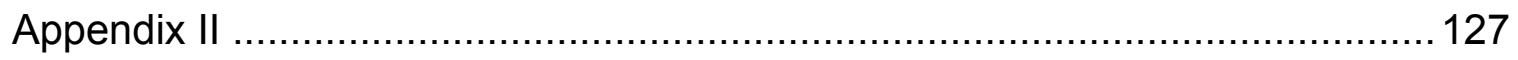

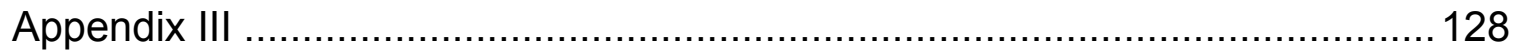

Standard Operating Procedures for Micro Force Displacement Machine ................. 128

Theory …

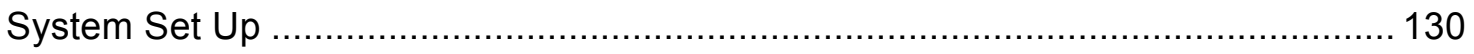

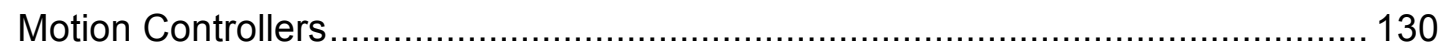

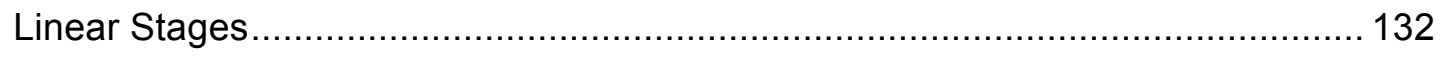

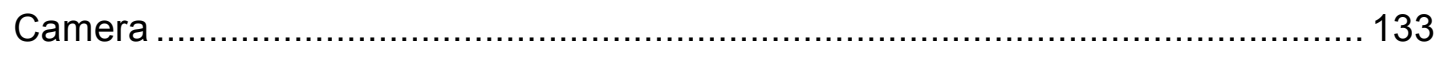

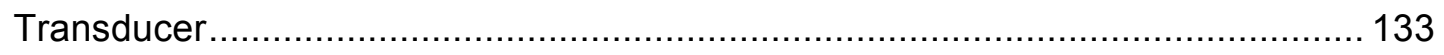

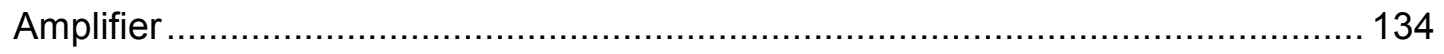

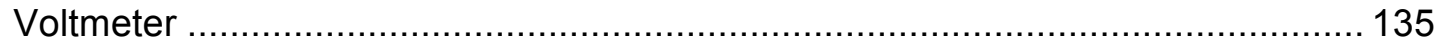

Testing

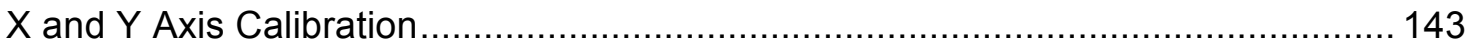

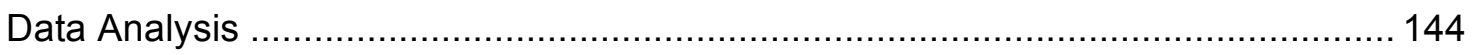




\section{LIST OF TABLES}

Table 1: Past Force Displacement Systems. ${ }^{2,13}$

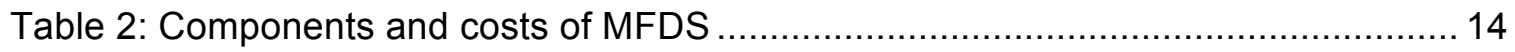

Table 3: RS-232 Commands for Individual Hardware Components ........................... 17

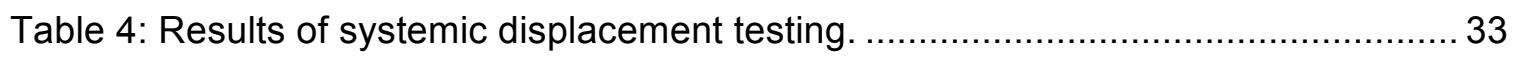

Table 5: Transfer function applied to systemic displacement slopes.......................... 37

Table 6: Diaphragm K values for A/B ratio of 1:1(Square Diaphragms) ${ }^{16} \ldots \ldots \ldots \ldots \ldots \ldots . \ldots 4$

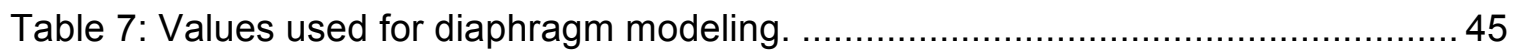

Table 8: Equivalent system spring constant produced from different sample sizes. ...... 46

Table 9: Comparison of Theoretical and Measured Results at Select Displacement

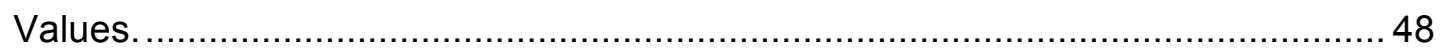

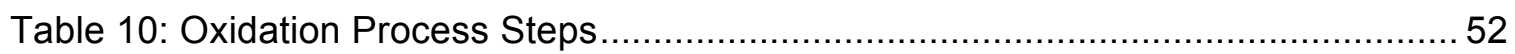

Table 11: Thickness of oxide grown on SOI process wafers.................................... 53

Table 12: Spin coating parameters for the application of positive photoresist...............56

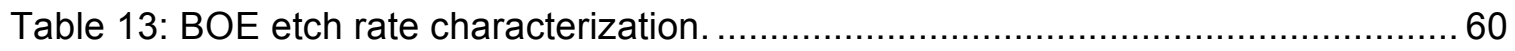

Table 14: Dual doped TMAH etch times for SOI process wafers. ............................... 68

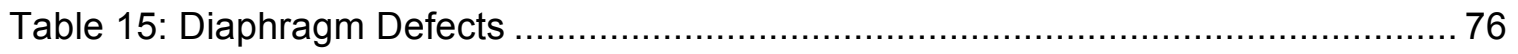


Table 16: $\mathrm{X}$ and $\mathrm{Y}$ lengths of diaphragms from both process wafers.

Table 17: Gage R\&R testing information for process wafer 1 and $2 \ldots \ldots \ldots \ldots \ldots \ldots \ldots \ldots \ldots . \ldots \ldots$

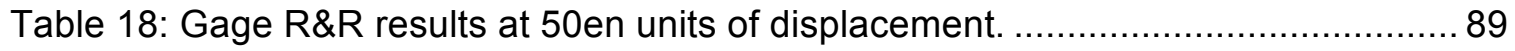

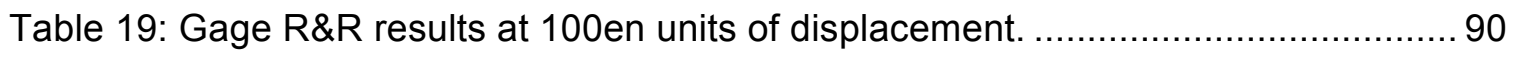

Table 20: Ev and Pv values for test values of 50 and 100en units of displacement...... 90

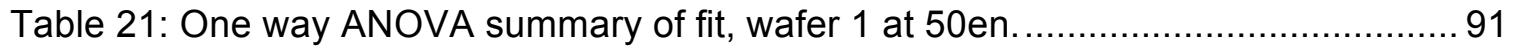

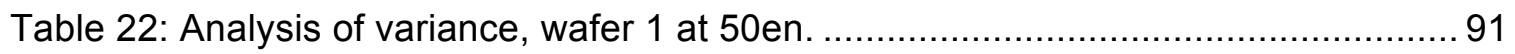

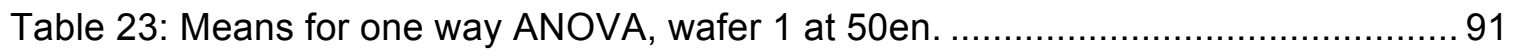

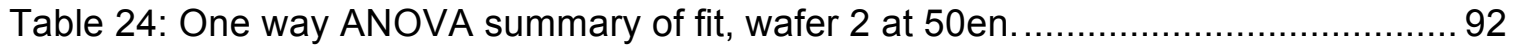

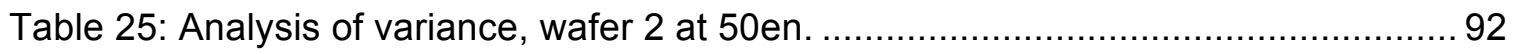

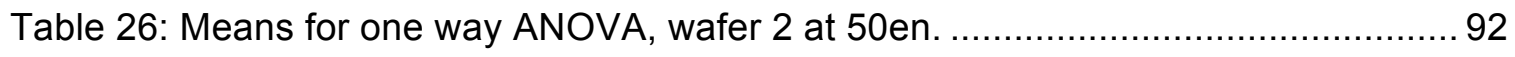

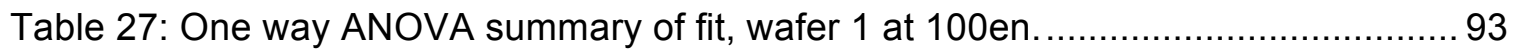

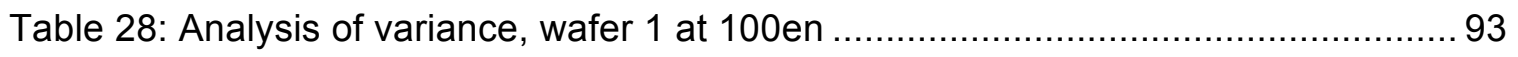

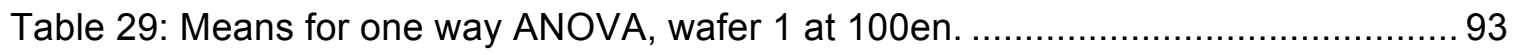

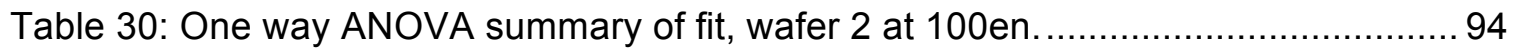

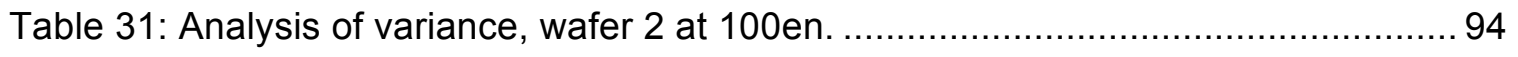

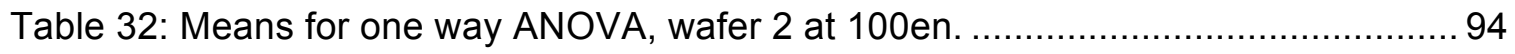

Table 33: Force Values at Select Displacement Values......................................... 95 


\section{LIST OF FIGURES}

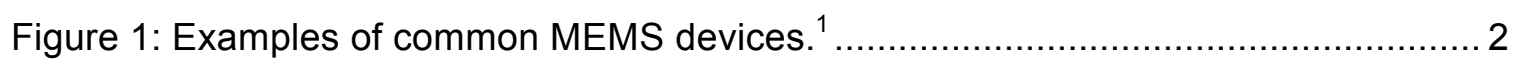

Figure 2: Example of a photolithography process used to create MEMS devices. ${ }^{4} \ldots \ldots \ldots . . . .3$

Figure 3: Example of bulk micromachining to create through holes, membranes, and v-

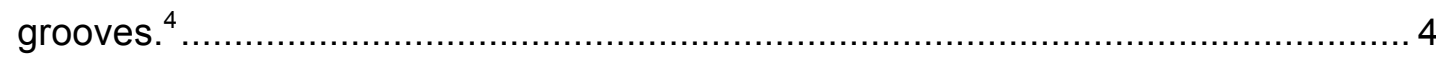

Figure 4: Example of surface micromachining to create a MEMS cantilever. ${ }^{4} \ldots \ldots \ldots \ldots \ldots 5$

Figure 5: Cross section of capacitive MEMS microphone. The capacitance between the top and bottom plate allow for the calculation of sound pressures. ${ }^{10} \ldots \ldots \ldots \ldots \ldots \ldots . . .6$

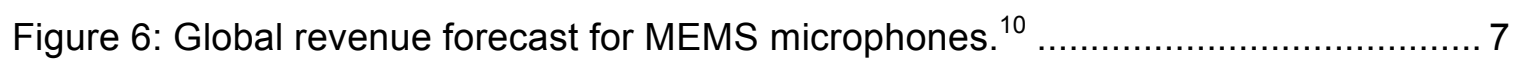

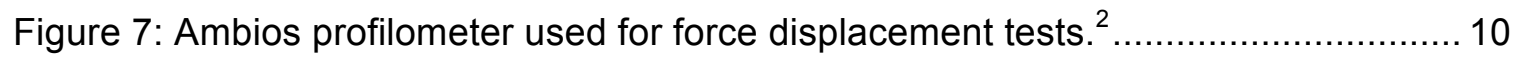

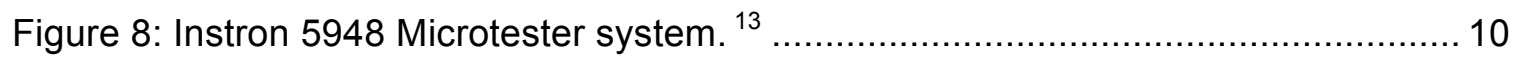

Figure 9: System block diagram of hardware design. Note: color-coding of blocks to

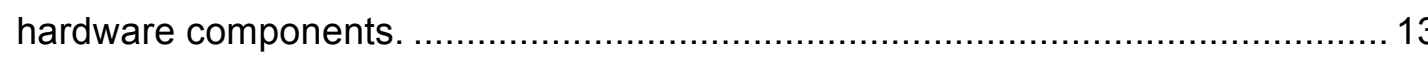

Figure 10: Transducer Subassembly: 1) Transducer body 2) Vented \#2-56 screw 3) Micro-prober tip. Interchangeable micro-prober tips allow future users the ability to tailor the system to their needs 
Figure 11: Rapid prototyped transducer loading fixture. This fixture allowed for transducer stability during tip loading which ensured the transducer did not exceed the specified load limit

Figure 12: Software GUI overview. See Appendix II for a larger view of the GUI 17

Figure 13: Software camera integration and image processing. 18

Figure 14: Software motion control of $X Y$ and $Z$ axes.

Figure 15: Software testing parameters as well as live transducer force and voltage readings. 20

Figure 16: Software live force displacement graph 20

Figure 17: Software calibration tools 21

Figure 18: Example of output CSV file. 22

Figure 19: Three major sources of displacement within the MFDS. 25

Figure 20: Consolidating individual displacements of MFDS components to one systemic displacement through the use of a rigid glass slide. 26

Figure 21: Plot of 59 runs of cyclic systemic displacement tests. The red lines represent the stage moving into the glass slide, while the blue lines represent the stage moving up from the glass slide. 28 
Figure 22: Six sigma confidence bounds of systemic displacement produced from 87 samples. The green line represents +3 sigma while the red line represents -3 sigma for each displacement unit. The black line represents the average systemic displacement slope. The blue region contains individual data points collected during each test run

Figure 23: Systemic displacement at low-end range of displacement. The green line represents the +3 sigma upper confidence interval, the red line represents the -3 sigma lower confidence interval, with the black line representing the average. The blue circles represent the individual data points from each test run.... 30

Figure 24: Histogram of voltage at 15en units of displacement. The data appears to be normally distributed. 30

Figure 25: Systemic displacement at middle range of displacement. The green line represents the +3 sigma upper confidence interval, the red line represents the -3 sigma lower confidence interval, with the black line representing the average. The blue circles represent the individual data points from each test run. 31

Figure 26: Histogram of voltage at 200en units of displacement. The data appears to be normally distributed 
Figure 27: Systemic displacement at high-end range of displacement. The green line represents the +3 sigma upper confidence interval, the red line represents the -3 sigma lower confidence interval, with the black line representing the average. The blue circles represent the individual data points from each test run.

Figure 28: Histogram of voltage at 330en units of displacement. The data appears to be normally distributed

Figure 29: Transfer function from 0 to $150 \mathrm{mg}$ of force. The test was run 3 times to collect more data on the transfer function

Figure 30: Linear regression produced from 3 test runs of the transducer transfer function.

Figure 31: Plot of residuals from transducer transfer function showing that the individual data points are normally distributed about the linear regression (although the residuals are large due to vibrations and the accuracy of the analytical balance). 36

Figure 32: Cyclic loading of transducer to characterize the warm up time of the system. 38

Figure 33: Wafer with aluminum layer sputtered on the backside of the wafer to create 1 um aluminum diaphragms. The dual doped TMAH solution has etched through the aluminum layer into the silicon, destroying the diaphragm material. .... 41 
Figure 34: Wafer with aluminum layer sputtered on the backside of the wafer creating 1 um aluminum diaphragms. The middle row of the diaphragms shown coincided with the etchant level in the etching chamber.

Figure 35: As received SOI process wafer with 400um handle side, 10um device side, and 6um buried oxide layer.

Figure 36: Location of different size diaphragms on photolithography mask used to create diaphragms. Red squares represent $4 \mathrm{~mm}$ diaphragms, green squares represent $5 \mathrm{~mm}$ diaphragms, and blue squares represent $7 \mathrm{~mm}$ diaphragms.

Figure 37: Force-displacement plot of 4,5 , and $7 \mathrm{~mm}$ diaphragms of $10 \mathrm{um}$ thickness from theoretical linear model.

Figure 38: Maximum stress at center of diaphragm for 4,5 , and $7 \mathrm{~mm}$ diaphragms of 10um thickness

Figure 39: Theoretical result of thermal oxidation processing.

Figure 40: Location of oxide growth measurements on handle side of SOI process wafers

Figure 41: SOI process wafer with positive photoresist applied to the handle side

of the wafer. 54

Figure 42: SOI process wafer with positive photoresist applied and patterned on the handle side of the wafer. 54

Figure 43: Cee 200X Spin Coater used for the application of positive photo resist. 55 
Figure 44: Hot plate used for soft and hard baking of wafers after the application of positive photo resist.

Figure 45: Lithography mask used to create 4,5 , and $7 \mathrm{~mm}$ diaphragms 57

Figure 46: Optical microscopy showing the diaphragm part of the lithography mask

as well as the photoresist protecting the oxide around each diaphragm. 58

Figure 47: Single sided BOE etch of SOI process wafer to selectively remove the thermally grown oxide on the device side of the SOI wafer.

Figure 48: Testing of BOE etch rate. 60

Figure 49: Single Sided etching apparatus used for BOE oxide etching. 61

Figure 50: Torque pattern to ensure no BOE leaked past the O-ring of the single sided etching apparatus. 62

Figure 51: BOE etch of oxide layer on handle side of SOI process wafer.

Diaphragms are visible as light purple squares, and positive photo resist is visible as the surrounding green areas. 62

Figure 52: Optical microscopy showing a close up of a diaphragm and break lines.

Photoresist is still masking the wafer.

Figure 53: Optical microscopy showing the diaphragm and break lines of the wafer after the positive photoresist had been removed from the wafer. 64 
Figure 54: SOI Process wafer after single sided BOE etch. The handle side of the SOI process wafer is shown. Grey regions represent bare silicon, while purple regions represent oxide. The second SOI process wafer (shown) had slight misalignment of the lithography mask making 3 of the diaphragms unusable (visible on the top and right side of the wafer). 64

Figure 55: Removal of positive photo resist from handle side of the SOI process wafer 65

Figure 56: A dual doped TMAH solution was used to selectively remove silicon from the handle layer of the SOI process wafers 66

Figure 57: Dual doped TMAH etching condenser chamber. 67

Figure 58: Visual inspection of vertically mounted SOI process wafer during dual doped TMAH etch. The buried oxide layer was visible once the handle side silicon had been fully removed. 68

Figure 59: Diaphragms after dual doped TMAH etch, warpage is visible due to the difference in film thickness of the buried and thermal oxide. 69

Figure 60: 3 dimensional plot generated from Bruker interferometer showing the warpage from the buried and thermal oxide layers. 70

Figure 61: Interferometer data from y direction of $7 \mathrm{~mm}$ diaphragm before the removal of the burried and themal oxide layers. The maximum warpage of this diaphragm was $67 \mathrm{um}$ 70 
Figure 62: Removal of thermally grown oxide on the handle side and device side of the wafer as well as the buried oxide beneath the etched silicon completed the wafer processing.......

Figure 63: 3 dimensional plot generated from Bruker interferometer showing the no warpage after the removal of the buried and thermal oxide layers. 72

Figure 64: Interferometer cross sectional scan data after buried and thermal oxides had been removed from the wafer. Curves in the data were caused by the stitching of many scans to create the overall size. The warpage is approximately $250 \mathrm{~nm}$, which is partially accountable through the weight of the diaphragm itself.

Figure 65: Example of a pinhole found on many of the diaphragms. 74

Figure 66: Diaphragm locations, $4 \mathrm{~mm}$ (red), 5mm (green), and $7 \mathrm{~mm}$ (blue)

diaphragms. 75

Figure 67: Pin hole location nomenclature. 75

Figure 68: Bar chart of location of defects based on region. 77

Figure 69: SEM cross-section of process wafer 2, the diaphragm thickness measured was $9.05 \mathrm{um}$. The image was taken at $15 \mathrm{kV}, 450 \mathrm{X}$ magnification, a working distance of $11 \mathrm{~mm}$, and a spot size of 20 . 78 
Figure 70: SEM cross-section from process wafer 2 showing the sidewall angle created from the dual doped TMAH etch. The relative thickness of the 10um diaphragm as compared to the 400 um handle layer of the SOI wafer is shown.

The image was taken at $15 \mathrm{kV}, 85 \mathrm{X}$ magnification, a working distance of $10 \mathrm{~mm}$, and a spot size of 35 79

Figure 71: SEM image showing etching non-uniformity on the sidewalls of process wafer 2, the yellow arrow points to the diaphragm, while the red arrows point to the sidewalls of the diaphragms. The image was taken at $30 \mathrm{kV}$, 140X magnification, a working distance of $21 \mathrm{~mm}$, and a spot size of 77 . 80

Figure 72: SEM image of process wafer 1 showing a close up of the diaphragm to handle layer interface of the SOI wafer. The arrow points to the buried oxide. The image was taken at $5 \mathrm{kV}, 430 \mathrm{X}$ magnification, a working distance of $12 \mathrm{~mm}$, and a spot size of 35 81

Figure 73: SEM image showing cross section of process wafer 1 . The image was taken at $5 \mathrm{kV}, 160 \mathrm{X}$ magnification, a working distance of $12 \mathrm{~mm}$, and a spot size of 20. 
Figure 74: SEM image from process wafer 1 showing undercutting from the dual doped TMAH solution under the oxide mask on the top side of the wafer. The image was taken at $5 \mathrm{kV}, 1900 \mathrm{X}$ magnification, a working distance of $12 \mathrm{~mm}$, and a spot size of 20 82

Figure 75: Breaking of the second process wafer to individually mount diaphragms. .... 84

Figure 76: Individually mounted diaphragm from the second process wafer.

Double-sided tape holds the diaphragm to a microscope slide. The diaphragm shown is number 6 , a $5 \mathrm{~mm}$ square diaphragm 84

Figure 77: Placement of test diaphragm beneath probe tip during Gage R\&R study..... 86

Figure 78: Amplifier used in Gage R\&R study, note the Output Level is currently below zero volts as indicated by the "Low" red LED. 86

Figure 79: Gage R\&R results from both process wafers and 3 different diaphragm sizes. 88

Figure 80: Gage R\&R results for process wafer 1. 88

Figure 81: Gage R\&R results for process wafer 2. 89

Figure 82: One way analysis of voltage by diaphragm size for process wafer 1 at 50 en units of displacement 91

Figure 83: One way analysis of voltage by diaphragm size for process wafer 2 at 50 en. 
Figure 84: One way analysis of voltage by diaphragm size for process wafer 1

at 100 en

Figure 85: One way analysis of voltage by diaphragm size for process wafer 2

at $100 \mathrm{en}$

Figure 86: Process wafer 1 Gage R\&R results as compared to theoretical model. 96

Figure 87: Process wafer 2 results from Gage R\&R study as compared to

theoretical model 96

Figure 88: Process wafer 1 gage R\&R results as compared to theoretical model

with correction formula.

98

Figure 89: Process wafer 2 gage R\&R results as compared to theoretical model

with correction formula. 99

Figure 90: Process wafer 2 gage R\&R results as compared to theoretical model

with correction formula and linear shift. 100

Figure 91: Transducer sub-assembly showing 1) transducer body, 2) vented screw, and 3) Micro-prober tip. 129

Figure 92: Configuration of reading delay and step size for testing 129

Figure 93: Block diagram of MFDS 130

Figure 94: ESP 300 Motion Controllers, with (1) on the top and (2) on the bottom..... 131 
Figure 95: Motion controller set up. From left to right: RS-232 input, Axis 2 input,

Axis 1 input, Power cord.

Figure 96: RS-232 to USB converters plugged into the computer. The blue USB

cable closest to the camera is for motion controller 1, while the blue USB

cable further from the camera is for motion controller 2.

132

Figure 97: Transducer assembly. 1) Transducer body 2) vented vacuum screw 3)

transducer tip.

133

Figure 98:Front of the World Precision Instruments TBM4M amplifier, note that

the Bridge Single Ended is switched to 'Diff' to remove noise from the output.

The plug in the front of the amplifier is the output from the transducer.

134

Figure 99: Back of World Precision Instruments TBM4M amplifier. The power switch

and power cable are shown. The BMC cable plugged into Output 1 will go to

the voltmeter. 135

Figure 100: Converting the BMC cable to banana cables. 135

Figure 101: Cables from amplifier plug into the front of the voltmeter. 136

Figure 102: Back of voltmeter, with RS-232 output (left) and power cable (right). 136

Figure 103: Location of Keithley 2400 input to computer. The Silver cable is the voltmeter input on the front of the computer. 137

Figure 104: Turning on motion controller 1 138 
Figure 105: Turning on motion controller 2

Figure 106: Turning on the Keithley 2400 voltmeter

Figure 107: Turning on the amplifier, the switch is top left as viewed from the

front of the amplifier.

Figure 108: Adjusting amplifier to achieve OV output as indicated by both the low and high LEDs lit 140

Figure 109: Video Capture Device selection, with DMK 72AUC02 selected as

the device name. 141

Figure 110: Initial plotting of output data from testing. 144

Figure 111: Data after transformations to create the proper units. 147 


\section{INTRODUCTION}

\subsection{PROBLEM STATEMENT}

The Materials Engineering Department of Cal Poly does not currently posses the ability to analyze the mechanical properties of MEMS devices created within the Microfabrication Lab. Previous projects have used a surface profilometer for mechanical testing with limited success at the price of profilometer damage. The profilometer uses a fixed mass (the mass of the profilometer tip and arm) to apply force to the silicon cantilever MEMS, while a photodiode array measures the angular displacement of a mirror used in calculating the linear displacement of the profilometer tip. The profilometer tip and arm apply a $0.25 \mathrm{mN}$ force to the cantilever, with a profilometer resolution of 20nm. A micro force displacement system (MFDS) needs to be designed to perform a force displacement test on devices such as 10um thick silicon diaphragms, silicon cantilevers, or other such devices of this scale. The system must be robust enough to stand up to years of use, as well as be easily and economically repairable by students in the future. The system should improve upon the profilometer technique of testing, with a force equal or less than $0.25 \mathrm{mN}$, with a resolution approaching $20 \mathrm{~nm}$.

\subsection{MEMS FABRICATION}

MicroElectroMechanical Systems (MEMS) are used in many industries for a wide array of applications. These devices are most often created from silicon, and utilize micro fabrication techniques to achieve device sizes on the micron scale. Some common uses for MEMS include pressure sensors, flow sensors, accelerometers, and micromirror arrays. Most of these MEMS devices rely on the physical actuation of silicon structures; for example accelerometers use the deflection of a cantilever beam to measure changes in acceleration. Common uses of these devices include monitoring tire pressure using the deflection of a diaphragm to quantify pressure change, monitoring 
chemical reaction rates by tracking the change in flow of thermal mass in a flow sensor, monitoring the deflection of a silicon cantilever to quantify acceleration, or producing inkjet nozzles with via holes (Figure 1).

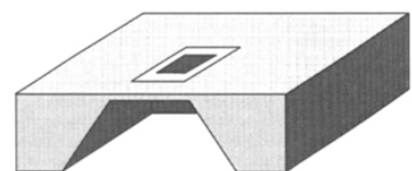

Pressure Sensors

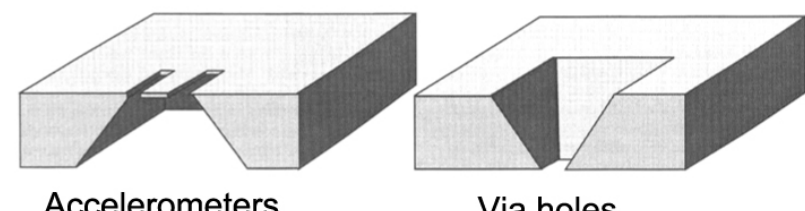

Figure 1: Examples of common MEMS devices. ${ }^{1}$

MEMS heavily utilize photolithography, surface micromachining, and bulk micromachining to form devices in a batch process, meaning hundreds or thousands of devices can be produced at once. ${ }^{2}$ Photolithography is a process by which selective etching of a substrate can be accomplished (Figure 2). The first step is the application of a photoresist, which "after exposure to UV light and subsequent developing, remains on the substrate" ${ }^{3}$. Using a mask, the UV exposure can be contained to certain areas of the photoresist creating a pattern once the photoresist has been developed. After step (d) in Figure 2, steps (e) and (g) represent one process, while (f) and (h) represent a different process. Steps (e) and (g) are used for the deposition of materials onto the substrate through the use of physical vapor deposition (PVD), chemical vapor deposition (CVD), and spin coating (among other processes). Steps (f) and (h) are used to selectively etch the substrate, in the case of silicon, an example of a common etchant is a $\mathrm{HNO}_{3} / \mathrm{HF}$ solution. ${ }^{3}$ 

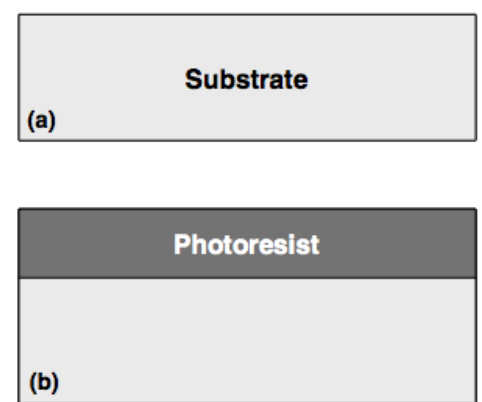

(b)
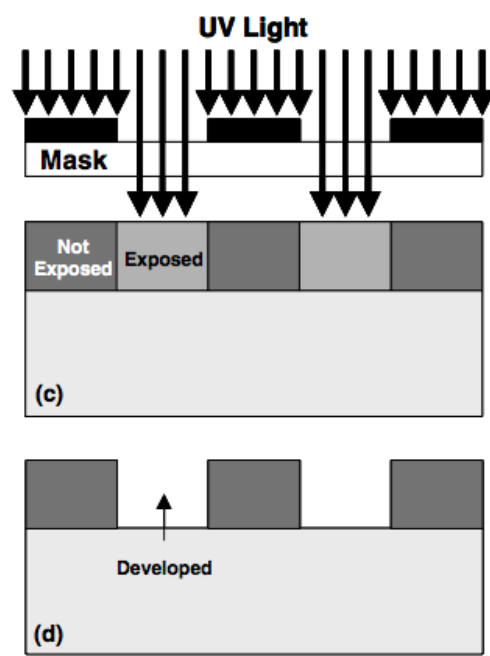
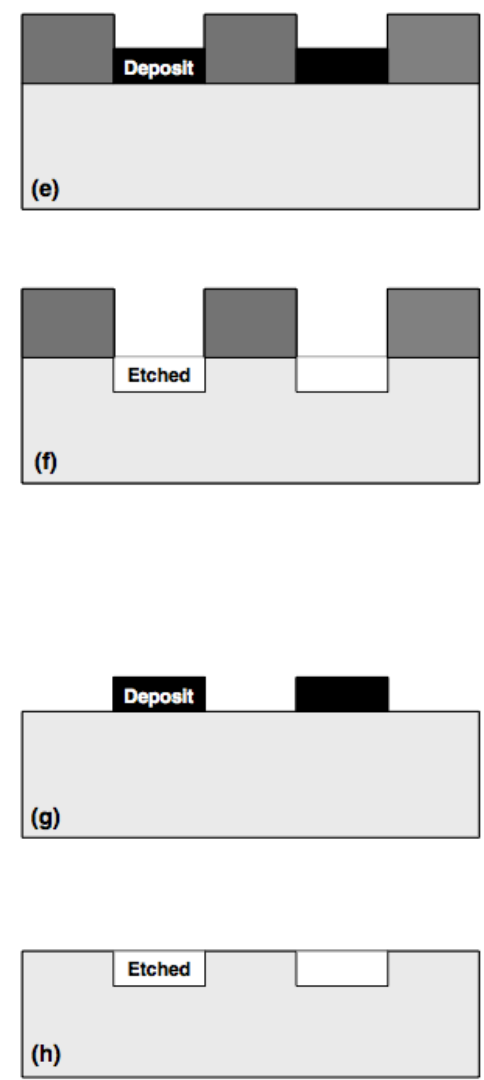

Figure 2: Example of a photolithography process used to create MEMS devices. ${ }^{4}$

As seen in step (h) of Figure 2, etchants can be used to selectively remove the substrate. Bulk micromachining can take this process further by utilizing the crystallographic planes of single crystalline silicon. Through the use of anisotropic etchants such as potassium hydroxide $(\mathrm{KOH})$ or tetra-methyl ammonium hydroxide (TMAH), a sidewall angle of $54.74^{\circ}$ can be achieved, allowing for structures such as through holes, membranes (for diaphragms), and v-grooves to be created (Figure 3 ). ${ }^{5}$ 

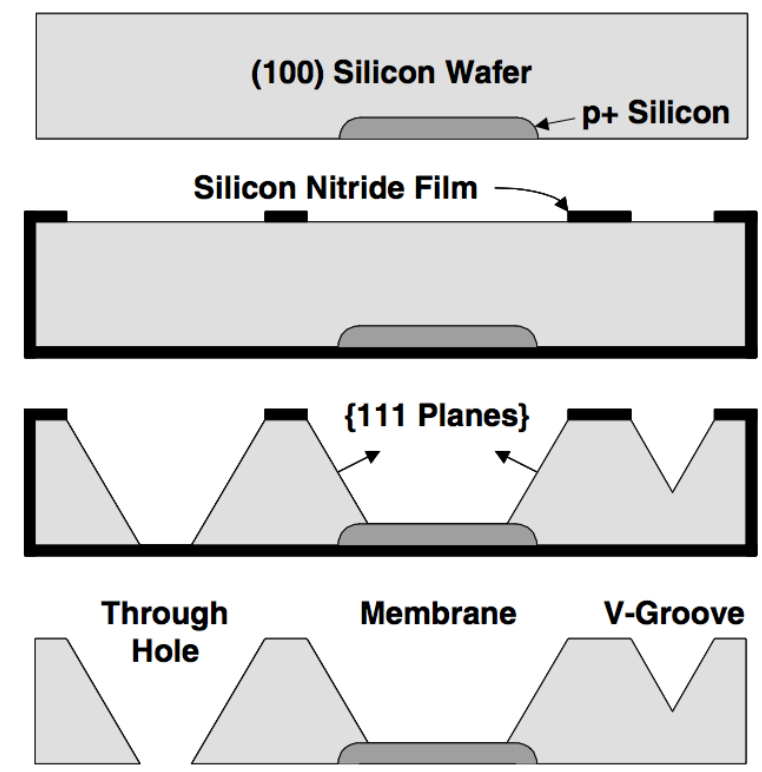

Figure 3: Example of bulk micromachining to create through holes, membranes, and v-grooves. ${ }^{4}$

Another important MEMS processing technique is surface micromachining, in which films for structural elements are deposited onto the surface of the substrate. Advantages of surface micromachining include higher yields (less waste due to set sidewall angle), and both lateral and vertical movement of components. ${ }^{5}$ Figure 4 demonstrates the creation of a cantilever for potential use as an accelerometer. First a sacrificial layer is deposited onto the substrate, next a structural layer (deposited using low pressure chemical vapor deposition (LPCVD) in the case of polysilicon) is deposited, and finally the sacrificial layer is removed from beneath the structural layer, leaving a freestanding structure. ${ }^{5}$ 


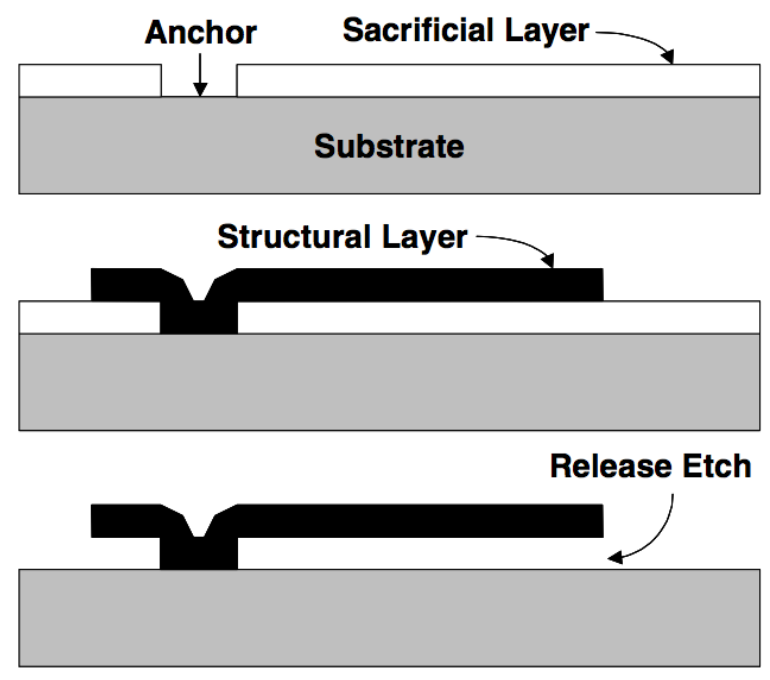

Figure 4: Example of surface micromachining to create a MEMS cantilever. ${ }^{4}$

Through the combination of these various techniques, a wide array of MEMS devices can be created. In a production setting, the device yield is high due to excellent process control and elevated cleanroom standards. Within the Microfabrication Lab at Cal Poly, more students lead to lower cleanroom standards and less process control. Subsequently, wafers have lower device yields, and the need to test and inspect individual MEMS devices.

\subsection{TEST STRUCTURES}

With the MEMS devices created in the lab, the most realistic way to test the MFDS would be to create structures of a similar scale and mechanical properties as these other MEMS devices. Previous work in the lab focused on the creation of electrostatically actuated silicon diaphragms ${ }^{6}$, however the processing sequence created was not able to produce thin enough diaphragms to enable measurable electrostatic actuation. 
Silicon diaphragms can serve in a variety of roles, the most common of which is a pressure sensor. MEMS pressure sensors have applications in a variety of fields, including: medical (blood pressure monitoring, intrauterine pressure monitors, inhalers, kidney dialysis, drug infusion pumps, etc. $)^{7}$, automotive (tire pressure sensors, oil pressure sensors, fuel tank vapor pressure, transmission fluid pressure, etc. $)^{8}$, and consumer electronics (microphones, speakers) ${ }^{9}$ MEMS microphones have the advantage of higher signal to noise ratios than that of conventional microphones, allowing for a broader dynamic sound range in consumer electronics (Figure 5).

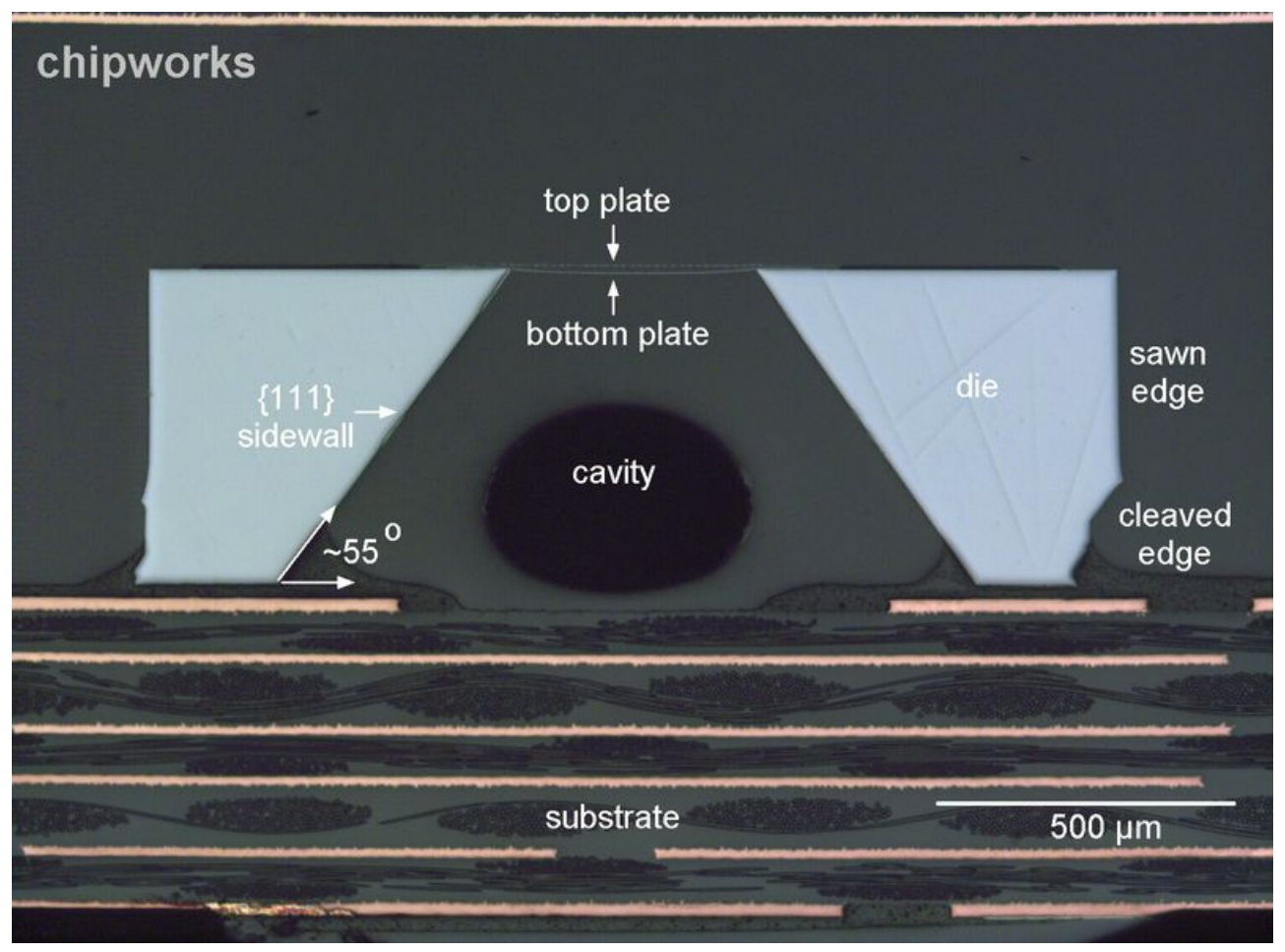

Figure 5: Cross section of capacitive MEMS microphone. The capacitance between the top and bottom plate allow for the calculation of sound pressures. ${ }^{10}$

With the popularity of cellphones and other consumer devices rising, the demand will only increase, leading to higher demand of high performance MEMS microphones (Figure 6). 


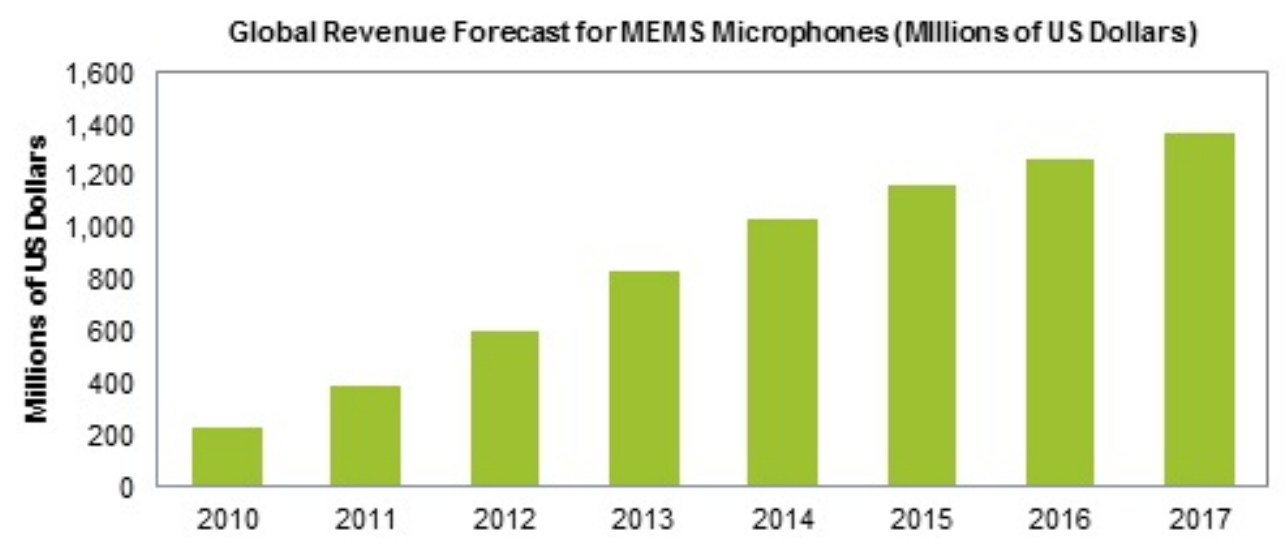

Source: IHS, March 2014

Figure 6: Global revenue forecast for MEMS microphones. ${ }^{10}$

The silicon diaphragm is one of the enabling technologies allowing for the creation of MEMS microphones. The popularity of these devices validates their use as test structures for this project, with the potential to use the processing steps from this thesis as the foundation for future projects to continue on to full MEMS microphone fabrication.

Square diaphragms with side lengths of 4,5 , and $7 \mathrm{~mm}$ were chosen to serve as test structures for the MFDS. The test structures would serve to compare theoretical results with observed results, as well as provide samples to perform a gage study to determine the resolution of the MFDS. 


\subsection{MOTIVATION}

Testing of final packaged devices (packaging refers to the material added to the MEMS device to protect it from environmental as well as provide electrical contact) is important to calibrate the devices prior to shipping to customers. Take an accelerometer for example, mechanical testing in the form of known accelerations are applied to the final device. The output of the accelerometer is then calibrated to the known acceleration to ensure proper function. ${ }^{11}$ These tests are easily performed on packaged devices.

Packaging and testing of all the final devices produced in the Microfabrication Lab would be prohibitively expensive and tedious process due to the additional equipment and labor required. A low device yield equates to throwing away many of the final packages produced due to bad MEMS devices within the packaging. Quantifying the physical properties of MEMS devices prior to packaging allows selective packaging of only properly functioning MEMS devices produced in the Microfabrication Lab. In addition, testing prior to final packaging allows the user to validate processing steps in addition to comparing the physical testing results to theoretical models.

Validating processing steps helps to ensure that proper etch rates are being observed for specific steps of processing. New etchants, old etchants, or etchants at different temperatures all produce different etch rates. If an etch rate is too slow, devices could be thicker than originally thought, and vice versa for faster etch rates. Quantifying this change through the observation of mechanical properties (deflection of a diaphragm etc.) allows the user to correct the processing step before moving to the subsequent step in processing. 
Often theoretical models are not accurate representations of MEMS devices due to thin film stresses introduced during processing (such as stresses introduced from high temperature deposition of materials and consequent cooling to room temperature). ${ }^{12}$ Physical testing of MEMS devices in the lab allows for a more accurate characterization of actual devices created. Identifying the differences between theoretical models and physical results can help to narrow the differences between the two, creating more accurate models in the future as well as a more complete understanding of material properties such as Young's Modulus.

\subsection{ForCE DisplaCEMENT SYSTEMS CURRENTLY AVAILABLE}

Current methods of inspecting mechanical properties of individual MEMS devices exist commercially, including an Ambios Profilometer in the Microfabrication Lab, as well as purpose built systems from companies like Instron (Table 1). On campus an Ambios Profilometer was used to apply a fixed force (the mass of the profilometer tip and arm) to MEMS devices to observe the displacement produced (Figure 7 ). ${ }^{2}$ The downside of this system is that if the force applied by the profilometer exceeds the capacity of the device, the device would break without data being collected. In addition the fixed force only gives one data point, not multiple force and displacement measurements, making the creation of a force displacement graph impossible. The profilometer was not designed to carry out force displacement measurements; as such the risk of damaging the profilometer is higher than that of a dedicated Microtester system.

Table 1: Past Force Displacement Systems. ${ }^{2,13}$

\begin{tabular}{|l|l|l|}
\hline System & Force Resolution & Displacement Resolution \\
\hline Ambios Profilometer & $0.28 \mathrm{mN}(2.85 \mathrm{mg})$ & $20 \mathrm{~nm}$ \\
\hline Instron 5948 Microtester & $<20 \mathrm{mN}(200 \mathrm{mg})$ & $20 \mathrm{~nm}$ \\
\hline
\end{tabular}




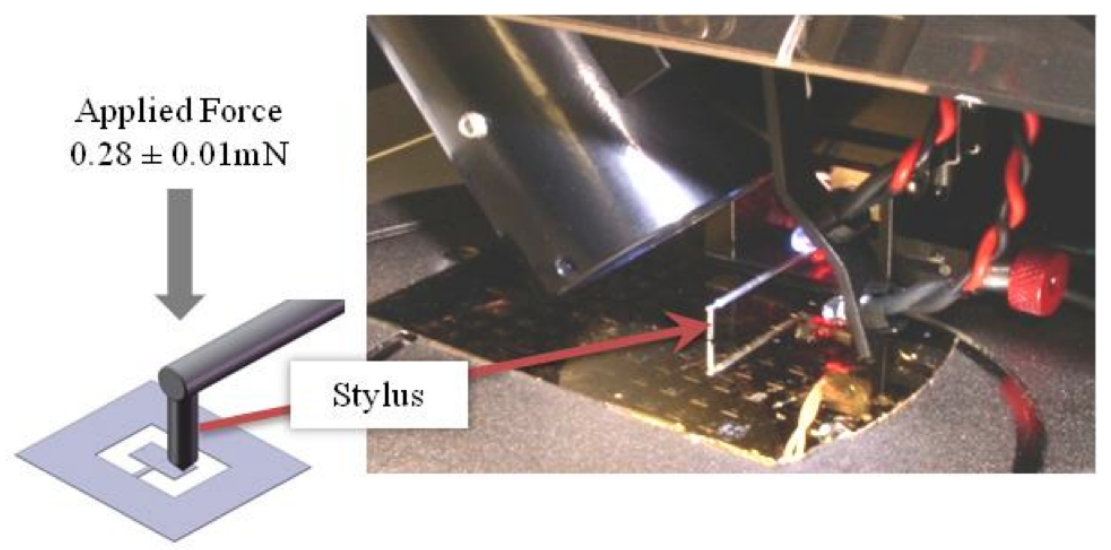

Figure 7: Ambios profilometer used for force displacement tests. ${ }^{2}$

The commercial side of force displacement testing at this scale is the Instron 5948 Microtester (Figure 8), which has a poor force resolution (Table 1), but comparable displacement resolution to the Ambios profilometer. One main issue with the Instron system is the mounting hardware involved to test a device. Wafer level devices would require a special mounting bracket to be tested.

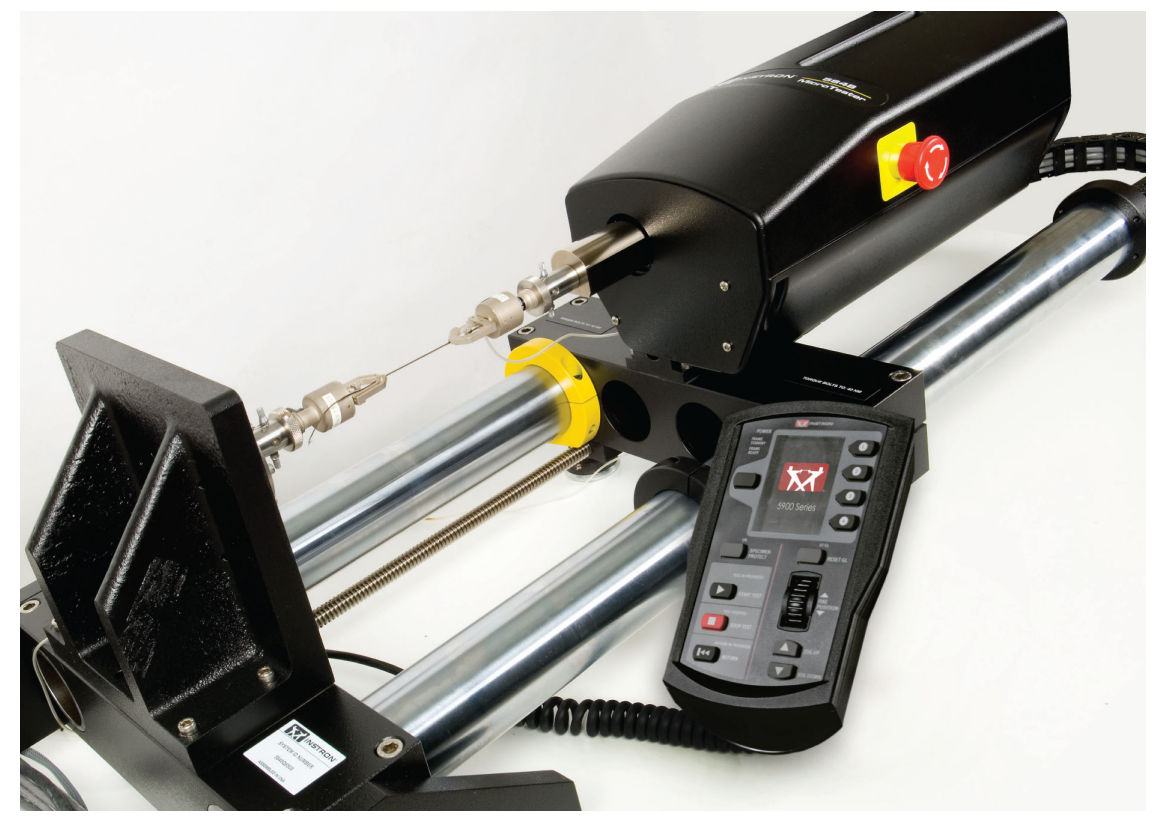

Figure 8: Instron 5948 Microtester system. ${ }^{13}$ 
Both of these systems are capable of recording force displacement data for MEMS devices created in the Microfabrication Lab, however a new system specifically designed for the devices created on campus would make it easier for students to collect data from MEMS devices.

\subsection{REALISTIC CONSTRAINTS}

Manufacturability:

One disadvantage of commercial force-displacement measurement systems such as the Instron 5948 is maintenance and consumables costs. Testing specimen fixtures as well as additional jigs represent supplementary income for companies like Instron, which for an educational institution signifies extra budget allocations for testing. Manufacturability of the MFDS was a chief concern, especially in the current budget crisis. The MFDS was designed to use mostly off the shelf components and standard fasteners, with the exception of two parts (both easily manufactured on campus). Additional testing specimen fixtures for the MFDS can be created using the rapid prototyping machine in the Materials Engineering Department, further reducing the cost of system.

\section{Economics:}

Testing MEMS devices during production allows the Materials Engineering Department and students to observe mechanical properties at key steps in production. If a certain process is not producing the desired effects, the MEMS devices can be reprocessed, reducing the probability of non-functioning devices being produced. Reducing the total number of devices produced for a given project saves the Materials Engineering Department money on process consumables including sputtering targets, photolithography supplies, and etchants among other supplies. Due to the hazardous 
nature of transporting some of these supplies, the cost of microfabrication can be extremely expensive, thus the economic benefit of producing the MFDS outweighs the capital cost of the system. 


\section{MFDS DESIGN}

\subsection{HARDWARE}

The MFDS incorporated both new equipment, and equipment used in other past projects in the Materials Engineering Microfabrication Lab. A systems block diagram was used to map system components (Figure 9).
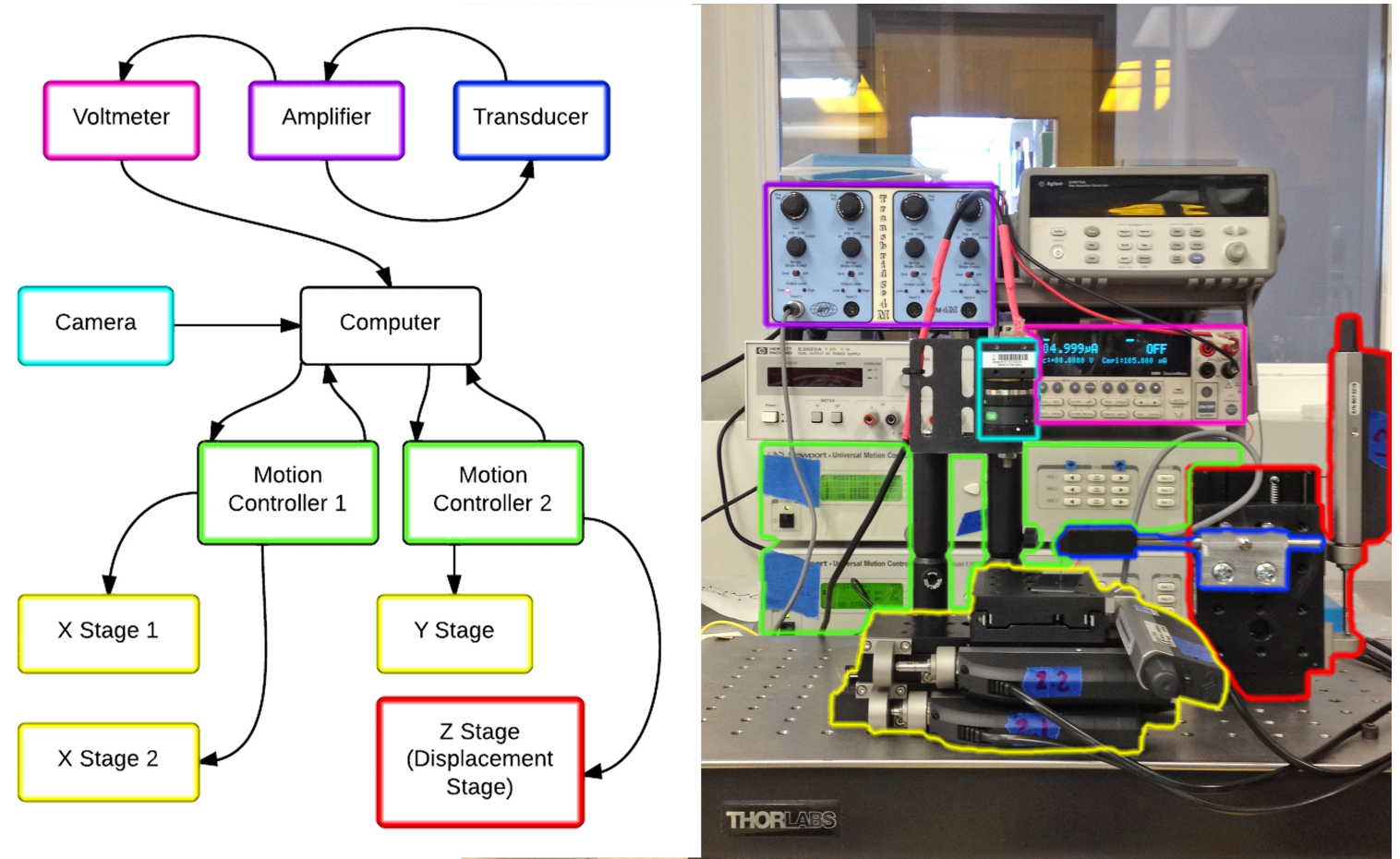

Figure 9: System block diagram of hardware design. Note: color-coding of blocks to hardware components.

A breakdown of components and associated costs was compiled (Table 2), using the costs to purchase a new component in the case of used equipment. The total approximant cost of the components for the system is $\$ 21,078$ for both the new equipment, and equipment already present in the Microfabrication Lab (used). 
Table 2: Components and costs of MFDS

\begin{tabular}{|l|l|l|l|}
\hline System Component & Actual Component & New/Used & Total Cost \\
\hline Computer & Dell & New & $\sim \$ 500$ \\
\hline Camera & $\begin{array}{l}\text { The Imaging Source DMK } \\
\text { 72AUC02 camera with M0814- } \\
\text { MP2 lens }\end{array}$ & New & $\$ 668$ \\
\hline Motion Controllers (2) & ESP300 & Used & $\sim \$ 5,800$ \\
\hline X Stages (2) & Newport M443 \& LTA-HS & Used & $\sim \$ 3,800$ \\
\hline Y Stage & Newport M443 \& LTA-HS & Used & $\sim \$ 1,900$ \\
\hline Z Stage & Newport M443 \& LTA-HS & Used & $\sim \$ 1,900$ \\
\hline Voltmeter & Keithley 2400 & Used & $\sim \$ 4,300$ \\
\hline Amplifier & $\begin{array}{l}\text { World Precision Instruments } \\
\text { TBM4M }\end{array}$ & New & $\$ 1,895$ \\
\hline Transducer & $\begin{array}{l}\text { World Precision Instruments } \\
\text { FORT10G }\end{array}$ & New & $\$ 315$ \\
\hline & & Total & $\sim \$ 21,078$ \\
\hline
\end{tabular}

The transducer and amplifier were the two most important new items to the system, allowing for the acquisition and processing of force observed during testing. Multiple options were considered to measure the force of the system, among those considered was using a constant weight while looking at displacement with capacitance, an AFM style transducer probe, a piezoelectric bending actuator, and a semiconductor strain gauge force transducer. Of these options, the semiconductor strain gauge force transducer was chosen for its cheap relative cost, accuracy, and resolution.

The transducer subassembly of the MFDS consisted of three parts; the transducer body, a vented \#2-56 screw, and a micro-prober tip (Figure 10). The utilization of a vented screw to connect the transducer to the micro-prober tip allowed future users to interchange tips based on the application during testing. The micro- 
prober tip was epoxied into the vented screw, which was then attached to the transducer body.

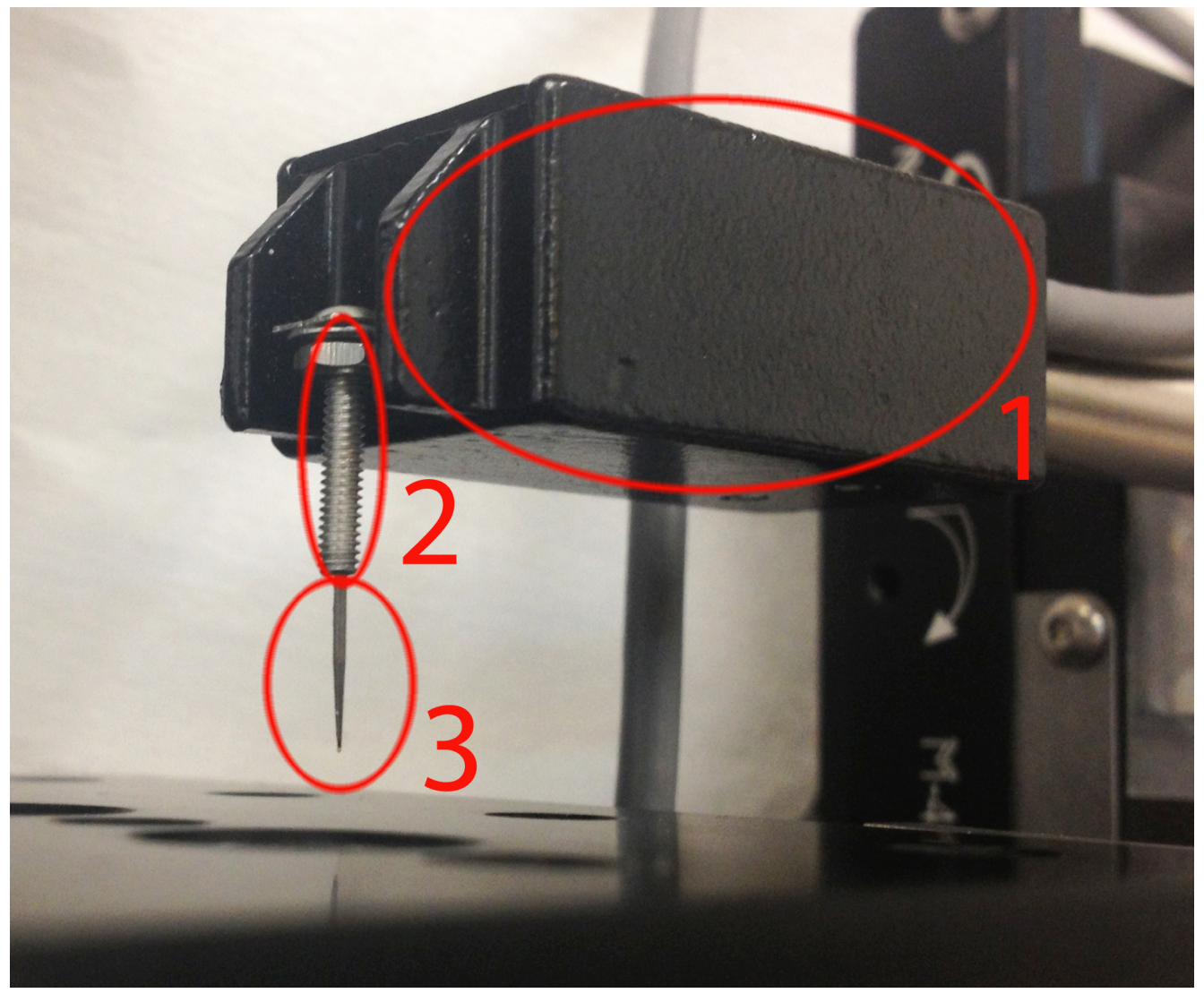

Figure 10: Transducer Subassembly: 1) Transducer body 2) Vented \#2-56 screw 3) Micro-prober tip. Interchangeable micro-prober tips allow future users the ability to tailor the system to their needs.

To load the micro-prober tip attached to the vented screw, a rapid prototyped ABS fixture was created to hold the transducer in place to ensure that the load limit of the transducer was not exceeded during attachment (Figure 11). The vertical post seen in Figure 11 mated with the bottom of the transducer cantilever, lending extra support to remain below the $20 \mathrm{~g}$ limit of the transducer. 


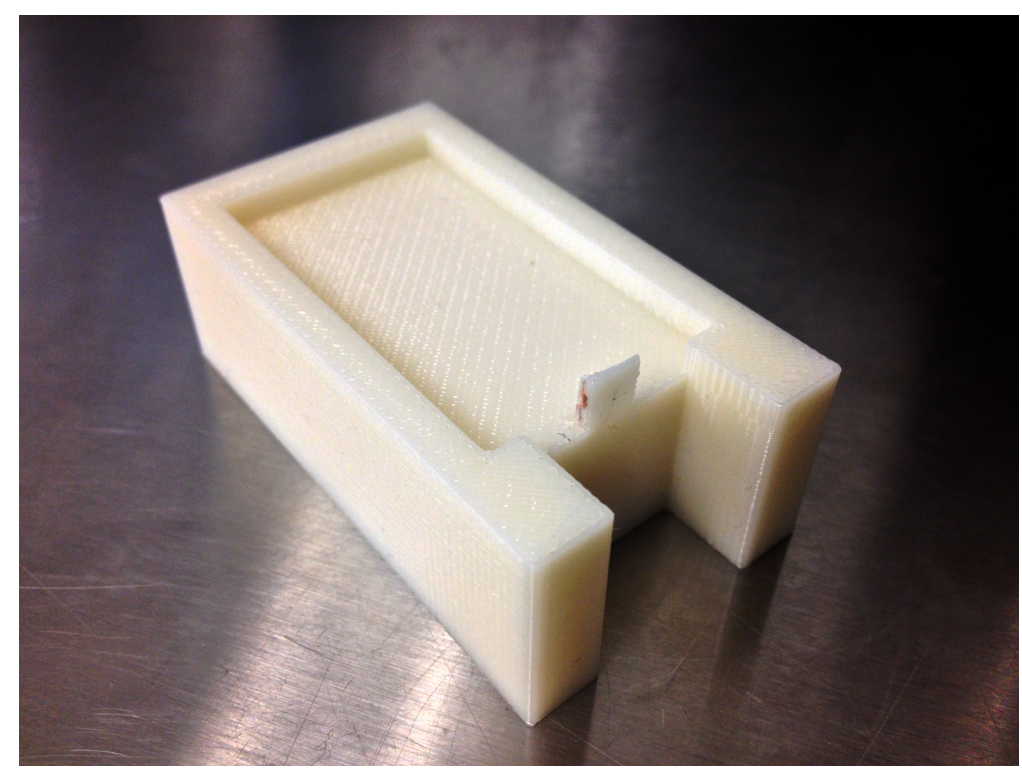

Figure 11: Rapid prototyped transducer loading fixture. This fixture allowed for transducer stability during tip loading which ensured the transducer did not exceed the specified load limit.

\subsection{SOFTWARE}

The software for this project was created with the help of Kevin Schapansky (CSC) who converted the functional requirements of the software into an integrated system. Initially LabView was chosen for the development environment for the project. However, due to compatibility issues with certain components (namely the ESP300 Motion Controllers), LabView could not be utilized. C\# provided the best alternative, with the capability to create a user-friendly graphic user interface (GUI). LabView provided built in functions available to the user, however in C\# individual RS-232 commands were sent to each of the hardware components. One component exception to communicating through RS-232 was the camera, which had a C\# library of functions available which allowed higher-level component integration into the GUI.

The RS-232 commands utilized in the MFDS were unique to each component, with software written for the motion controllers, voltmeter, and analytical balance. 
Examples of RS-232 commands and their functions are shown in Table 3. For a complete list of commands used, reference Appendix 1.

Table 3: RS-232 Commands for Individual Hardware Components

\begin{tabular}{|l|l|l|}
\hline Component & Machine Code & Function \\
\hline ESP 300 Motion Controller & 1PR10 & $\begin{array}{l}\text { Move axis 1 10 positive units } \\
\text { relative to the current position }\end{array}$ \\
\hline Keithley 2400 & :READ? & Read voltage \\
\hline Analytical Balance & !KP & Read balance \\
\hline
\end{tabular}

After the integration of the components into the software, a GUI was created to serve as an interface for the end user (Figure 12). The interface allows full control of the system components as well as testing parameters.
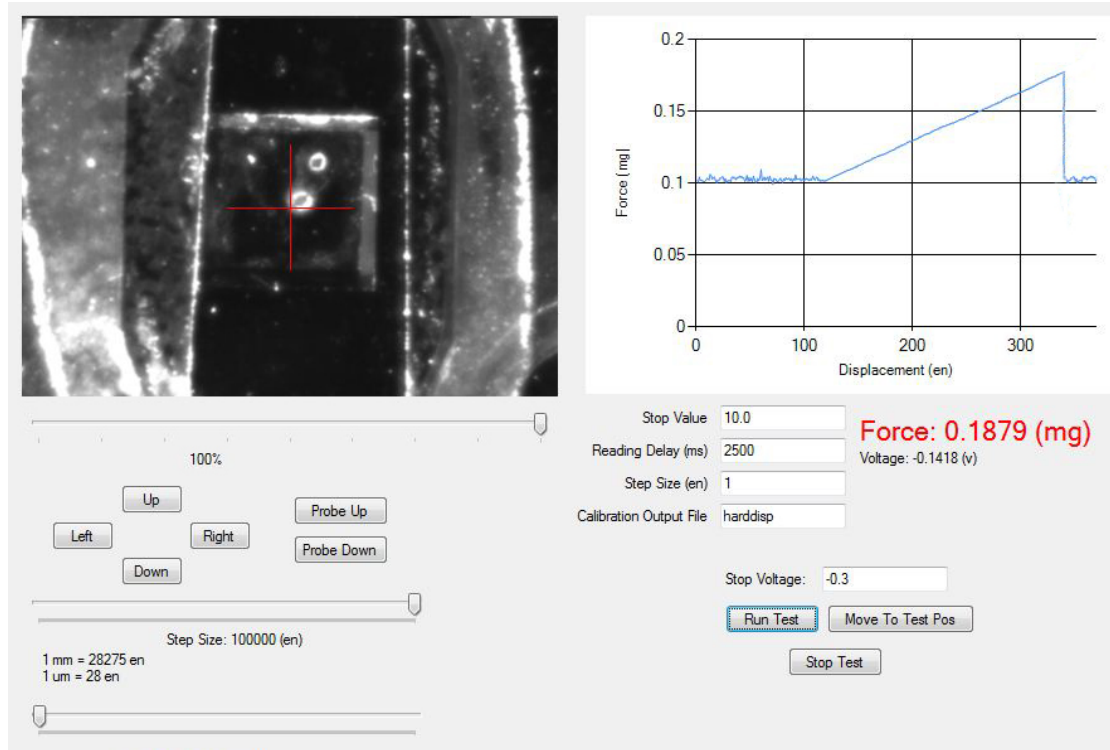

— Forcevs. Displacement

Vertical Step Size: 96 (en)

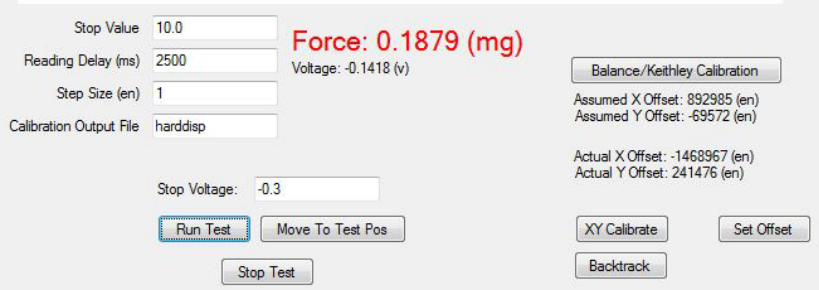

Figure 12: Software GUI overview. See Appendix II for a larger view of the GUI

The software can be broken down into individual components and the respective functions of each. The first section of the software is the image from the camera and camera controls (Figure 13). The camera view allows the user to see the sample being 
tested as well as zoom in or out on the sample using the slider beneath the image. The red crosshairs visible in the center of the camera view represent the portion of the sample on which the test will take place. The slider provides a reading of the zoom level of the camera; Figure 13 shows the current zoom level at $100 \%$.

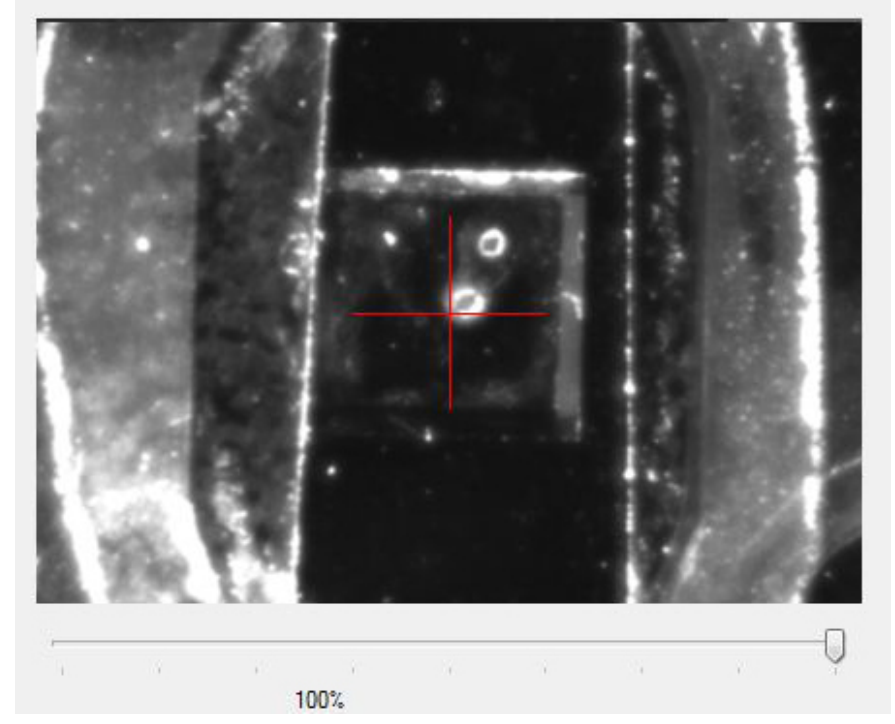

Figure 13: Software camera integration and image processing.

Beneath the imaging control is the motion control section of the GUI (Figure 14). This section allows the user to control the 4 total stages of the system. The two X-axis stages are controlled using the "Left" and "Right" buttons as well as the top slider labeled "Step Size". The Y-axis stage is controlled using the "Up" and "Down" buttons as well as the "Step Size" slider. The Z-axis stage is controlled using the "Probe Up" and "Probe Down" buttons as well as the "Vertical Step Size" slider. It should be noted that the "Step Size" slider controls the motion step size for both the $\mathrm{X}$ and $\mathrm{Y}$-axis stages. Encoder units (en) were chosen as the displacement step sizes, which each represent approximately $35 \mathrm{~nm}$ of displacement per step. 


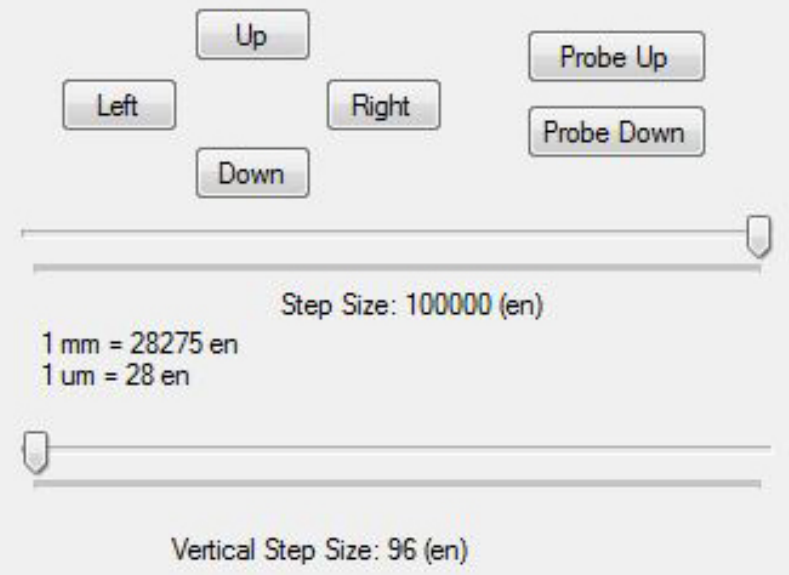

Figure 14: Software motion control of $X Y$ and $Z$ axes.

The testing is initiated through the software and allows the user to input various test parameters designed to protect the test samples as well as the transducer of the MFDS. The testing parameters include the stopping voltage on the transducer, reading delay, and step size (Figure 15). In addition the testing parameter section allows the user to define the output filename and location. The live force and voltage the transducer is experiencing is displayed as a preventative measure against breaking the transducer. The "Move to Test Pos" button utilizes the offset found in Figure 17 to move the sample from under the camera cross hairs to a calibrated position under the transducer tip. The offset is user definable and consists of $X \& Y$ axis units of movement that can change based on the setup of the system. Should the force on the transducer approach the force limit, the test can be stopped utilizing the "Stop Test" button. The transducer force limit is 10 grams, however the user should not exceed 5 grams of force during testing. The Keithley 2400 was later confirmed not to be the limiting factor of force sensing, as it was capable of testing $\pm 11 \mathrm{~g}$ at full amplification. 


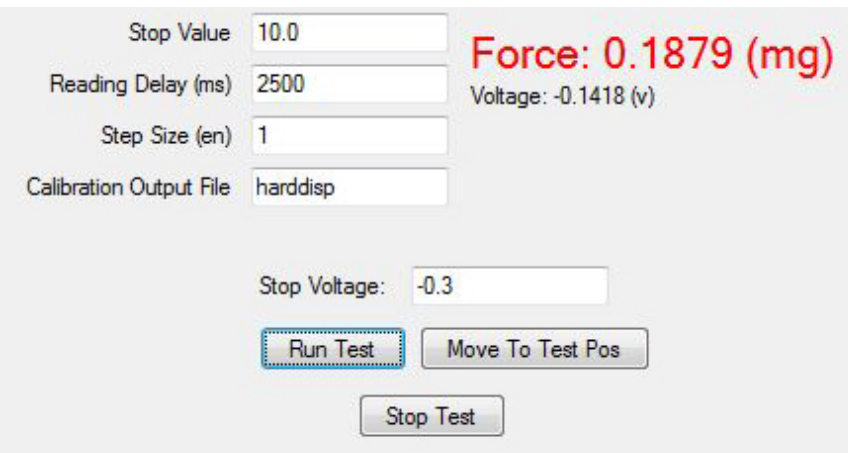

Figure 15: Software testing parameters as well as live transducer force and voltage readings.

Once the test has been initiated, a live graph displaying the force and displacement of the test is produced (Figure 16). The graph is not for data processing purposes (it does not take into account systemic displacement) but is instead used as a testing metric to ensure the transducer does not experience excessive force. In addition the graph is also used as a tool to stop tests once the sample has broken. In Figure 16 the sharp drop in force at approximately 350 en displacement units represents the loaded sample being broken. Once this behavior is observed in the graph, the user should stop the test to prevent damage to the transducer.

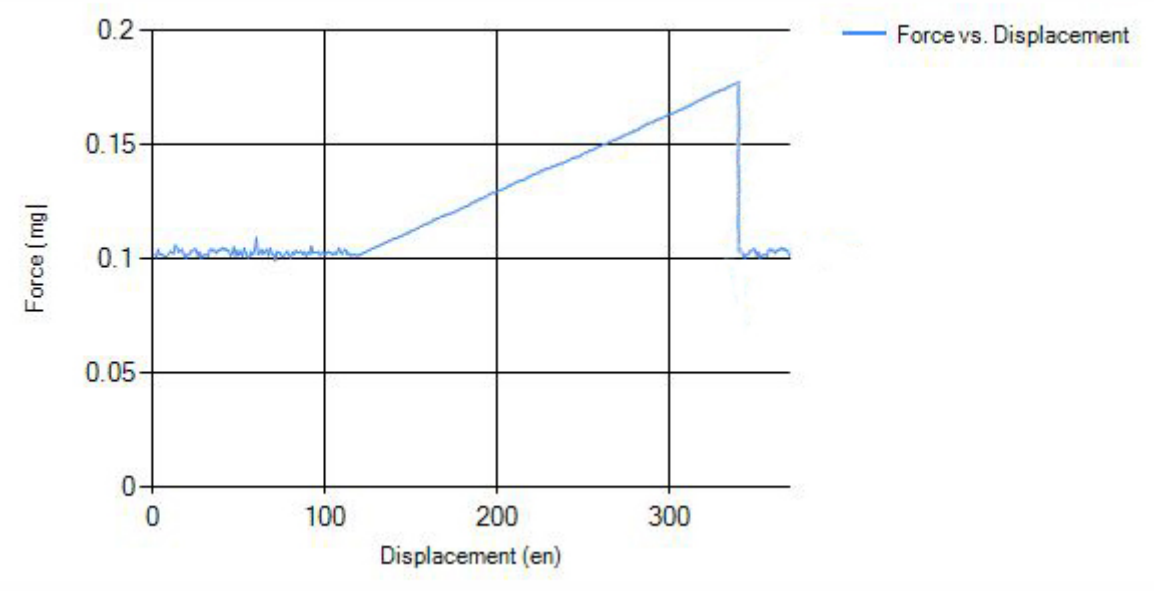

Figure 16: Software live force displacement graph 
Prior to any testing, the system must be calibrated in several ways. For a complete explanation of system calibration, see the CALIBRATION section. The calibration tools included in the software encompass calibration for the transducer, and the $\mathrm{X}$ and $\mathrm{Y}$-axis offset calibration (Figure 17). The "Balance/Keithley Calibration" allows the user to characterize the voltage and force produced when the transducer is brought into contact with an analytical balance. The "XY Calibrate" button allows the user to define the offset from the crosshairs on the camera to the actual tip of the transducer. The assumed $\mathrm{X}$ and $\mathrm{Y}$-Axes offset represents the current offset (in encoder units) that the system perceives is correct. Once the user has realigned the $\mathrm{X}$ and $\mathrm{Y}$-Axis (see $\mathrm{X}$ \& Y AXIS CALIBRATION section) the actual offset reflects any changes from the initial offset the system has generated. As visible in Figure 17 the actual and assumed offset for both the $\mathrm{X}$ and $\mathrm{Y}$-Axes are presented to the user for reference. The "Backtrack" button is the opposite of the "Move to Test Pos" button, which is used to move the sample from beneath the transducer tip to the cross hairs of the fiducial camera view. Using the "Backtrack" button, the point source used for calibration can be moved from the transducer to the camera (and vice versa) to ensure the $\mathrm{X} \& \mathrm{Y}$-Axis calibration was successful.

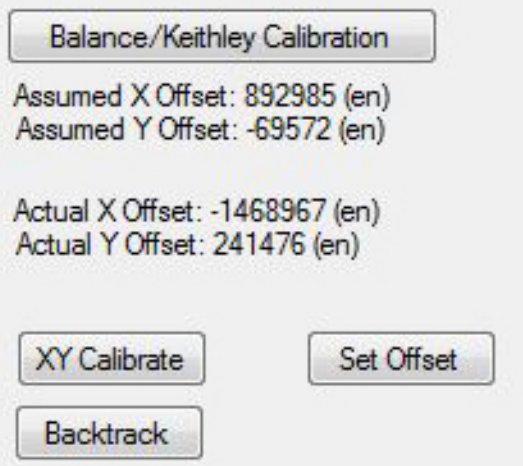

Figure 17: Software calibration tools 
Once a test is completed, the system outputs a comma separated value (CSV) file with a header indicating the date, time, and testing parameters of the test (Figure 18). The CSV file only outputs the displacement in encoder units (en) and the voltage observed, both of which must be converted to physical units using equations found through calibrating the $\mathrm{z}$ axis displacement, systemic displacement, and transducer force.

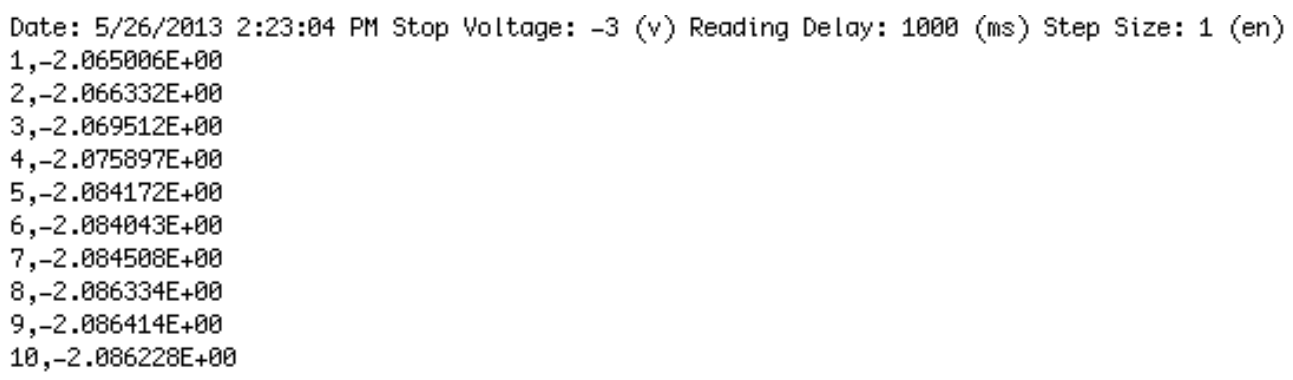

Figure 18: Example of output CSV file. 


\section{Calibration}

\subsection{X \& Y AXIS CALIBRATION}

In order for the user to utilize the cross hairs of the camera view to align samples, the offset from the center of the camera to the tip of the transducer must be known by the system. To calibrate the $X$ and $Y$ stages, a small amount of silicone grease was placed on the transducer tip. A glass microscope slide was then placed onto the testing specimen area. The tip of the transducer was brought into contact with the glass slide, and then lifted off. The "XY Calibrate" button was then selected. At this point the software used the assumed offset to bring the silicone grease "dot" close to the center of the camera view. The $X$ and $Y$ stages were then used to position the dot under the crosshairs of the camera. Once satisfied with the position, the "Set Offset" button was selected. Through the course of calibration, the software tracked the movements made by the user and added or subtracted the displacement values from the assumed offset to produce the actual offset. Once the calibration was complete, a sample could be placed into the MFDS and aligned using the crosshairs. The "Move To Test Pos" button could then be pressed to move the sample from the camera crosshairs to the transducer tip. Repeating this process multiple times insured that the $x$ and $y$ stages returned to the same position each time during calibration. If the transducer tip is replaced, or the system is dismantled and reassembled, the $X \& Y$ axis calibration must be performed to insure correct alignment.

\subsection{Z AXIS DISPLACEMENT CALIBRATION}

The resolution of the linear stages of the MFDS was specified as $35.367 \mathrm{~nm}$ from the manufacturer. To ensure that this displacement specification was correct, the $Z$ stage was calibrated using a reticle calibration stage micrometer, with 100um increments. The $Z$ stage was placed below the camera (in the same plane as the $X$ or $Y$ 
stages making movement parallel to the camera sensor surface), and then the stage was moved 28281 en units (equal to $1 \mathrm{~mm}$ or 10 steps of the reticle calibration stage micrometer). The stage aligned exactly with the $1 \mathrm{~mm}$ mark of the reticle micrometer, ensuring that the encoder units specified were correct. The displacement data was then used to convert the raw output encoder units to displacement in microns. For all future calculations, a translation of 28.3 encoder units per micron was used to convert the displacement in native units to microns. Future calibration could be accomplished through the placement of the stage under an optical microscope for a more accurate view of the microscope reticle micrometer, although the clearance of optical microscopes in the Microfabrication Lab did not support this form of testing.

\subsection{SYSTEMIC DISPLACEMENT CHARACTERIZATION}

The transducer used in the MFDS flexed as it created a voltage. When a sample is loaded into the MFDS, there are three main sources of displacement in the system: the transducer body, transducer tip, and the test sample. All three of the sources share

the same applied force, but differ in their individual displacements due to the spring constants of the individual sources of displacement (Figure 19). 


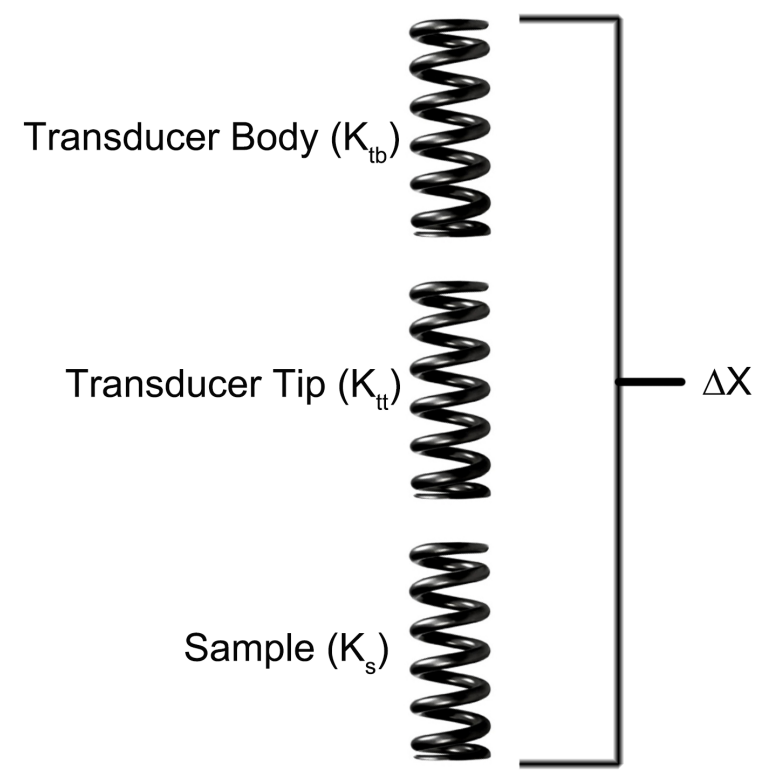

Figure 19: Three major sources of displacement within the MFDS.

Separating each spring from the system, the spring constants of the components can be found (Eq. 1). $\mathrm{K}_{\mathrm{tt}}$

$$
K_{t b}=\frac{F}{\Delta X_{t b}} \quad K_{t t}=\frac{F}{\Delta X_{t t}} \quad K_{s}=\frac{F}{\Delta X_{s}} \quad K_{\text {system }}=\frac{F}{\Delta X_{\text {total }}}
$$

Eq. 1

Flipping the equations and adding the components together, the individual spring constants can be related to the spring constant of the system (Eq. 2-Eq. 4).

$$
\begin{aligned}
& \frac{\Delta X_{t b}}{F}+\frac{\Delta X_{t t}}{F}+\frac{\Delta X_{s}}{F}=\frac{\Delta X_{\text {total }}}{F} \\
& \frac{\Delta X_{t b}+\Delta X_{t t}+\Delta X_{s}}{F}=\frac{\Delta X_{\text {total }}}{F}
\end{aligned}
$$

Eq. 3 


$$
\frac{1}{K_{t b}}+\frac{1}{K_{t t}}+\frac{1}{K_{s}}=\frac{1}{K_{\text {system }}}
$$

Eq. 4

To determine the displacement of the sample $\left(\Delta X_{s}\right)$, the displacement of the other parts must be characterized. The systemic displacement $\left(\Delta \mathrm{X}_{\mathrm{tb}}+\Delta \mathrm{X}_{\mathrm{tt}}\right)$ was determined by placing a glass side in the sample area. The spring constant of the glass slide was assumed to be infinite, leaving the observed displacement to be equal to the systemic displacement. The displacement produced within the transducer $\left(\Delta \mathrm{X}_{\mathrm{tt}}+\Delta \mathrm{X}_{\mathrm{tb}}\right)$ was characterized by applying a known voltage to a rigid glass slide to isolate the displacement associated with the transducer $\left(\Delta \mathrm{X}_{\mathrm{tt}}+\Delta \mathrm{X}_{\mathrm{tb}}\right)$.

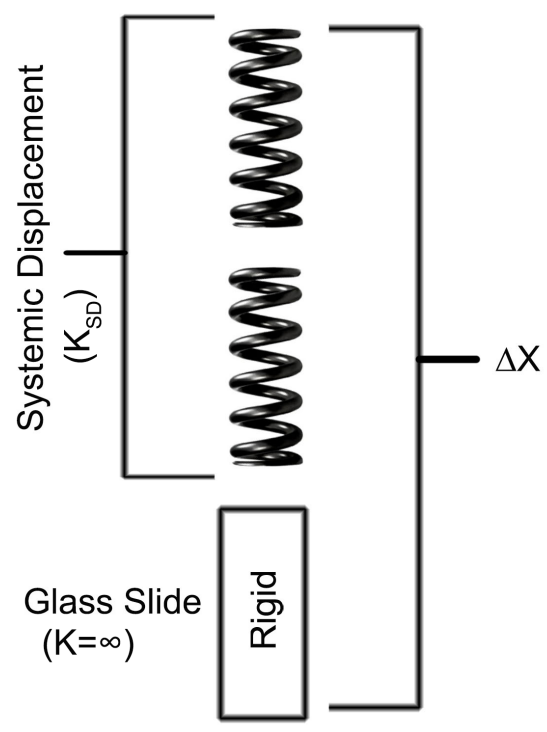

Figure 20: Consolidating individual displacements of MFDS components to one systemic displacement through the use of a rigid glass slide.

From Eq. 4, the components of the series of spring constants consolidates to two components, the systemic displacement (Eq. 5) and glass slide (Eq. 6). 


$$
\begin{gathered}
K_{S D}=K_{t b}+K_{t t} \\
\frac{1}{K_{S D}}+\frac{1}{K_{\text {glass slide }}}=\frac{1}{K_{\text {system }}}
\end{gathered}
$$

Eq. 6

Assuming that the spring constant of the glass slide is infinite, the glass slide term becomes zero, reducing the equation (Eq. 7).

$$
\frac{1}{K_{S D}}=\frac{1}{K_{\text {system }}}
$$

Eq. 7

The systemic displacement $\left(\Delta \mathrm{X}_{\text {total }}\right)$ was then characterized in the form of a relationship between a voltage value and the corresponding displacement value. Initial tests were performed to examine if there was a difference in systemic displacement between positive or negative Z-axis motion. 59 paired runs were performed, and the graphed data indicates that the system is stiffer with positive Z-axis motion (the transducer being lowered into the sample) as opposed to negative Z-axis motion (the transducer being raised from the surface of the sample)(Figure 21). Subsequent testing was only performed with the transducer driven into the sample, to reduce the error associated with the systemic displacement testing. 


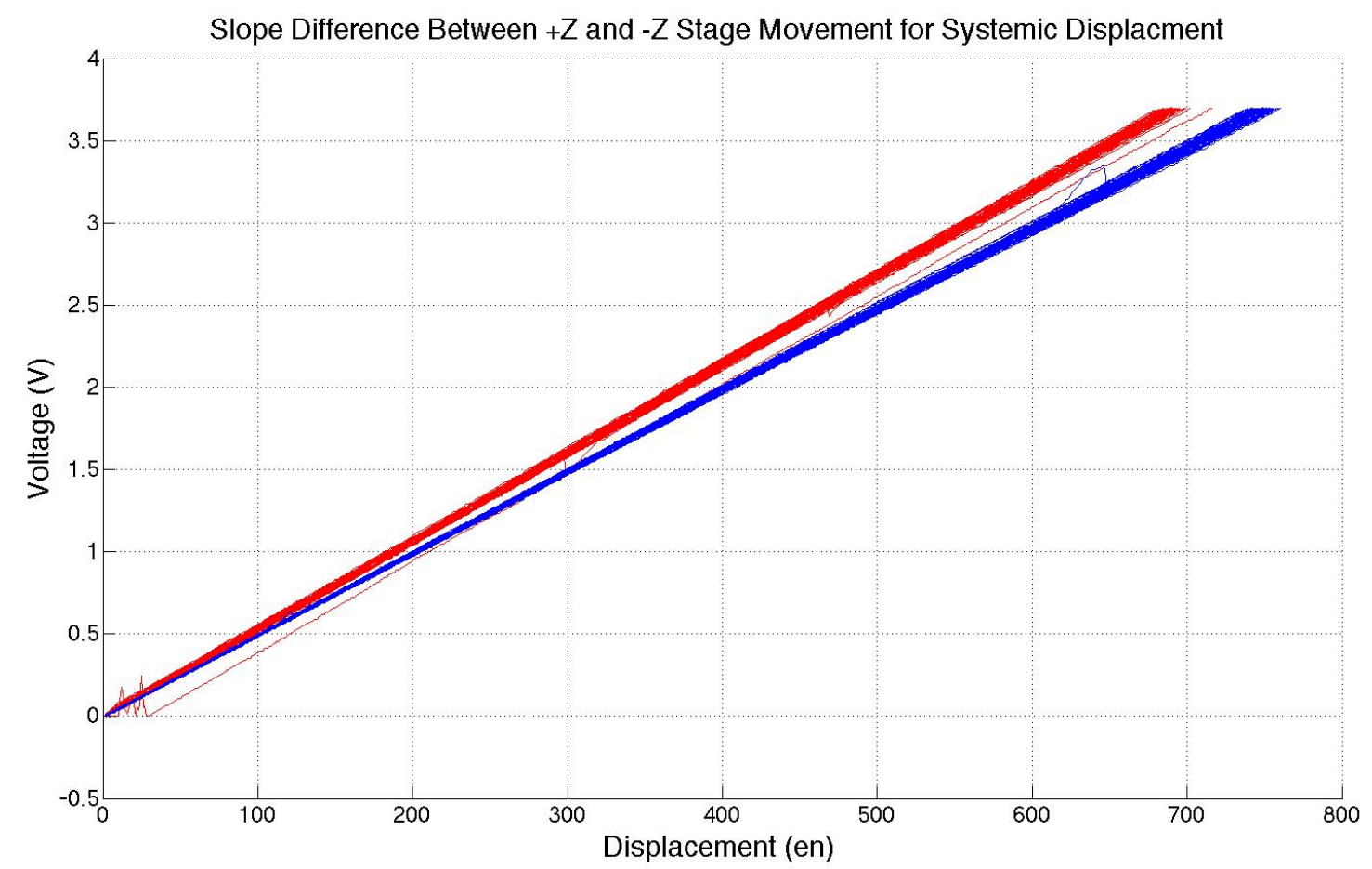

Figure 21: Plot of 59 runs of cyclic systemic displacement tests. The red lines represent the stage moving into the glass slide, while the blue lines represent the stage moving up from the glass slide.

Next the transducer was lowered onto a glass slide; 87 samples were collected with a $1000 \mathrm{~ms}$ delay between each step, with a step size of 1en (Figure 22). 


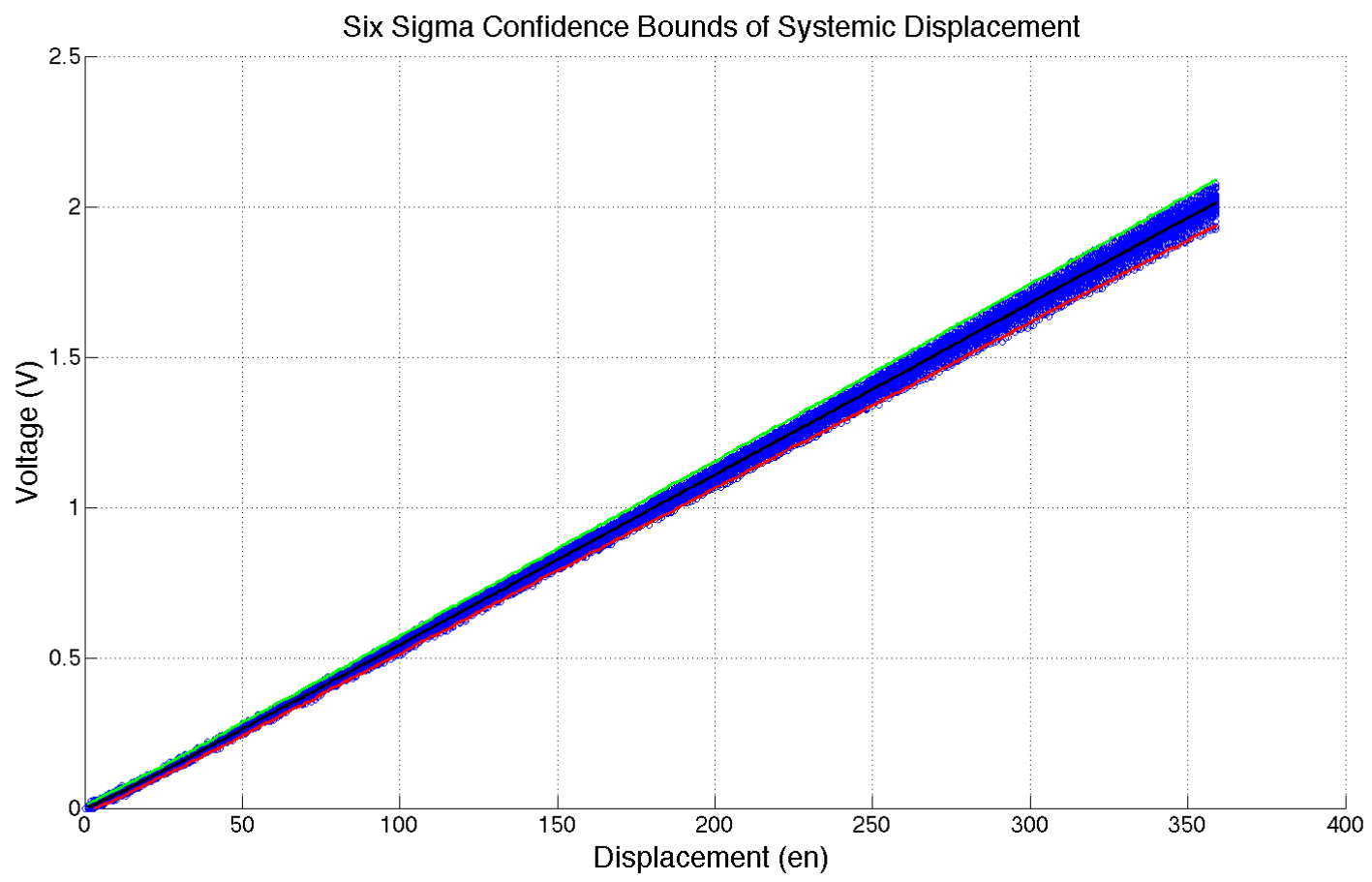

Figure 22: Six sigma confidence bounds of systemic displacement produced from 87 samples. The green line represents +3 sigma while the red line represents -3 sigma for each displacement unit. The black line represents the average systemic displacement slope. The blue region contains individual data points collected during each test run.

In order to use a standard deviation approach to determining the upper and lower confidence bounds of the systemic displacement spring constant, the data must have a normal distribution. The data was analyzed at 15,200 , and 330 en units of displacement, corresponding to the low, middle, and high end of the data range (Figure 23, Figure 25, and Figure 27). Histograms of the data were taken at 15, 220, and 330en units of displacement to determine if the data was normally distributed. Examining the data it was determined that a normal distribution was the most appropriate fit (Figure 24, Figure 26, and Figure 28). 


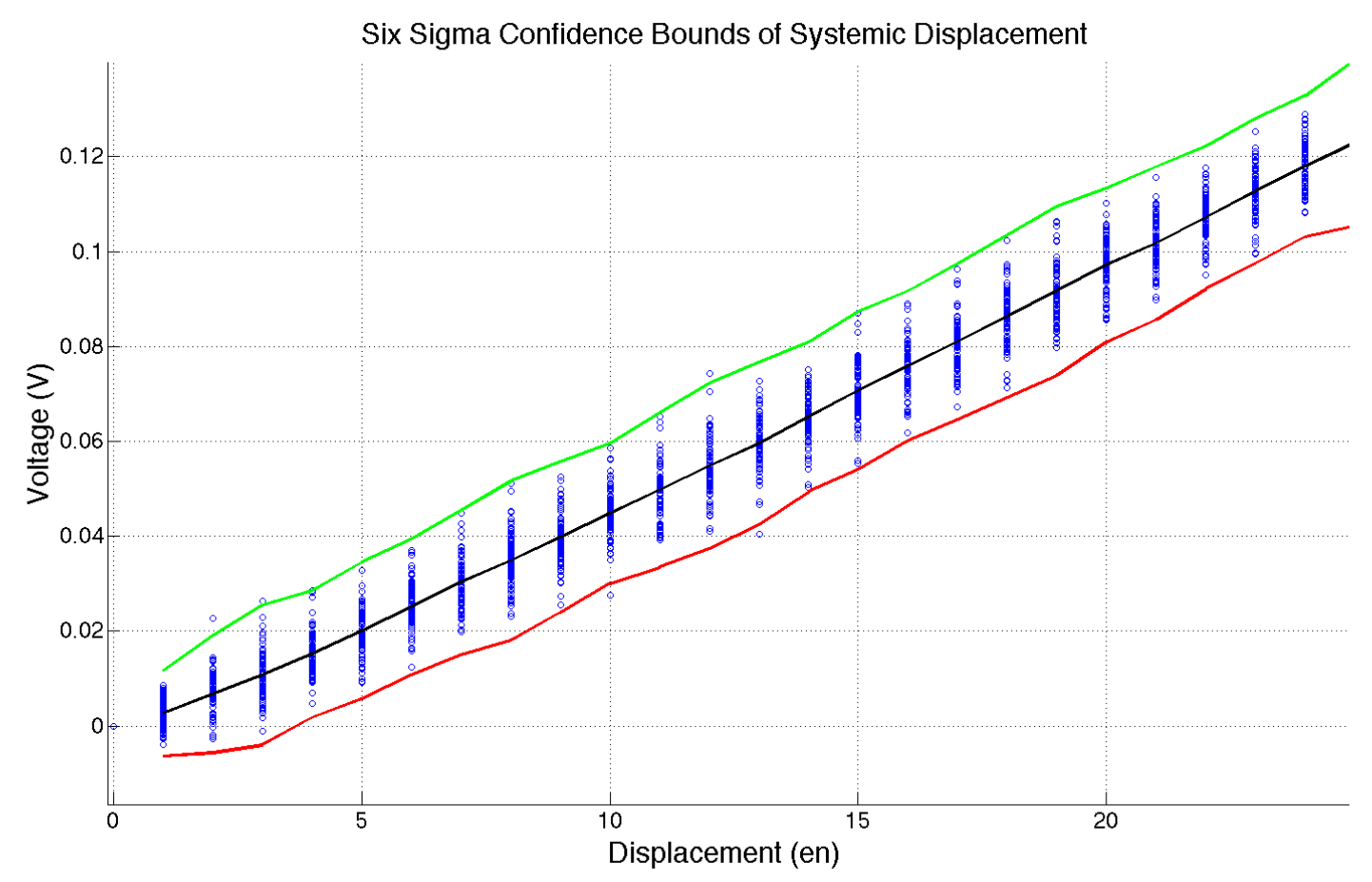

Figure 23: Systemic displacement at low-end range of displacement. The green line represents the +3 sigma upper confidence interval, the red line represents the -3 sigma lower confidence interval, with the black line representing the average. The blue circles represent the individual data points from each test run.

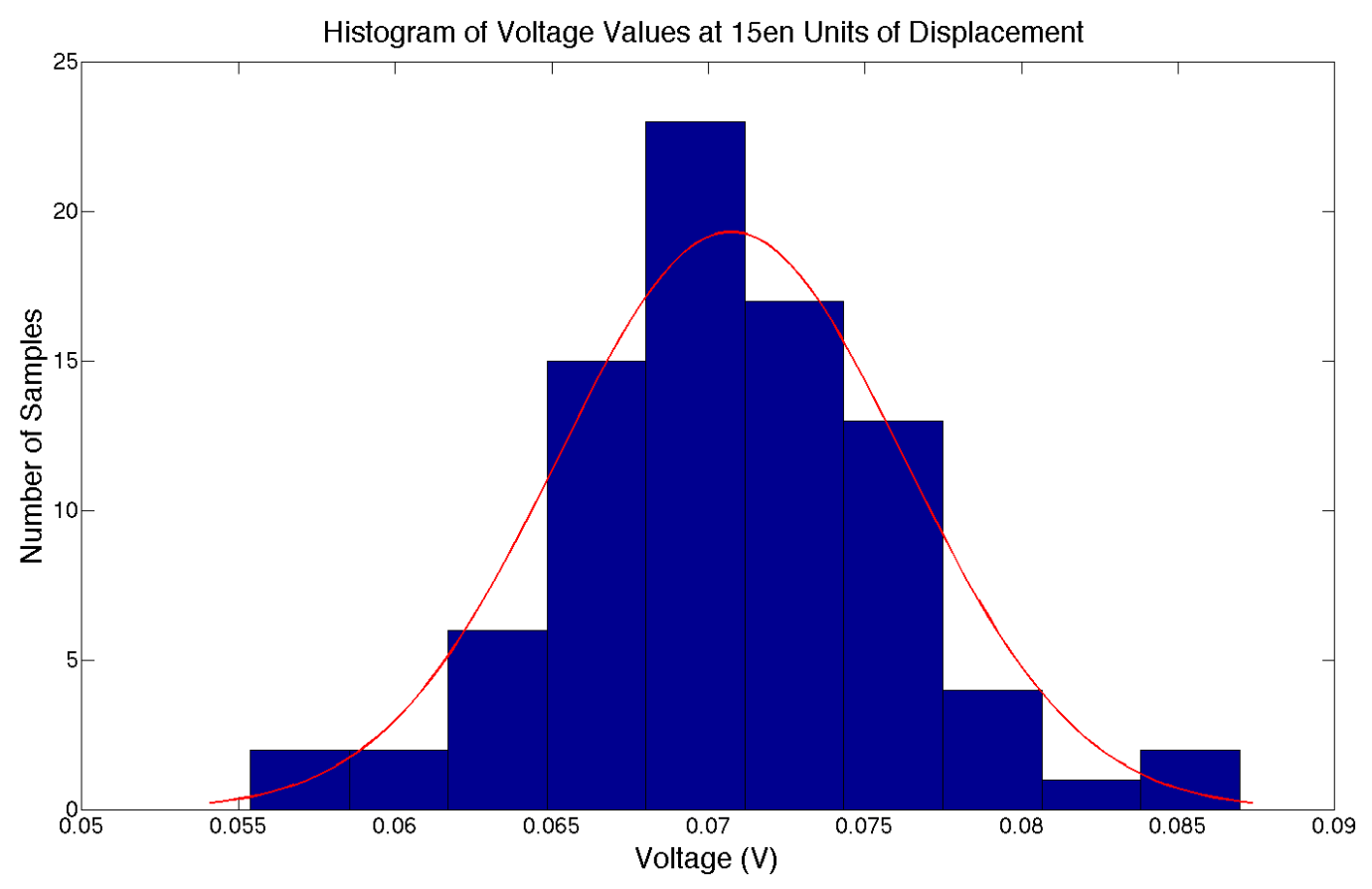

Figure 24: Histogram of voltage at 15en units of displacement. The data appears to be normally distributed. 


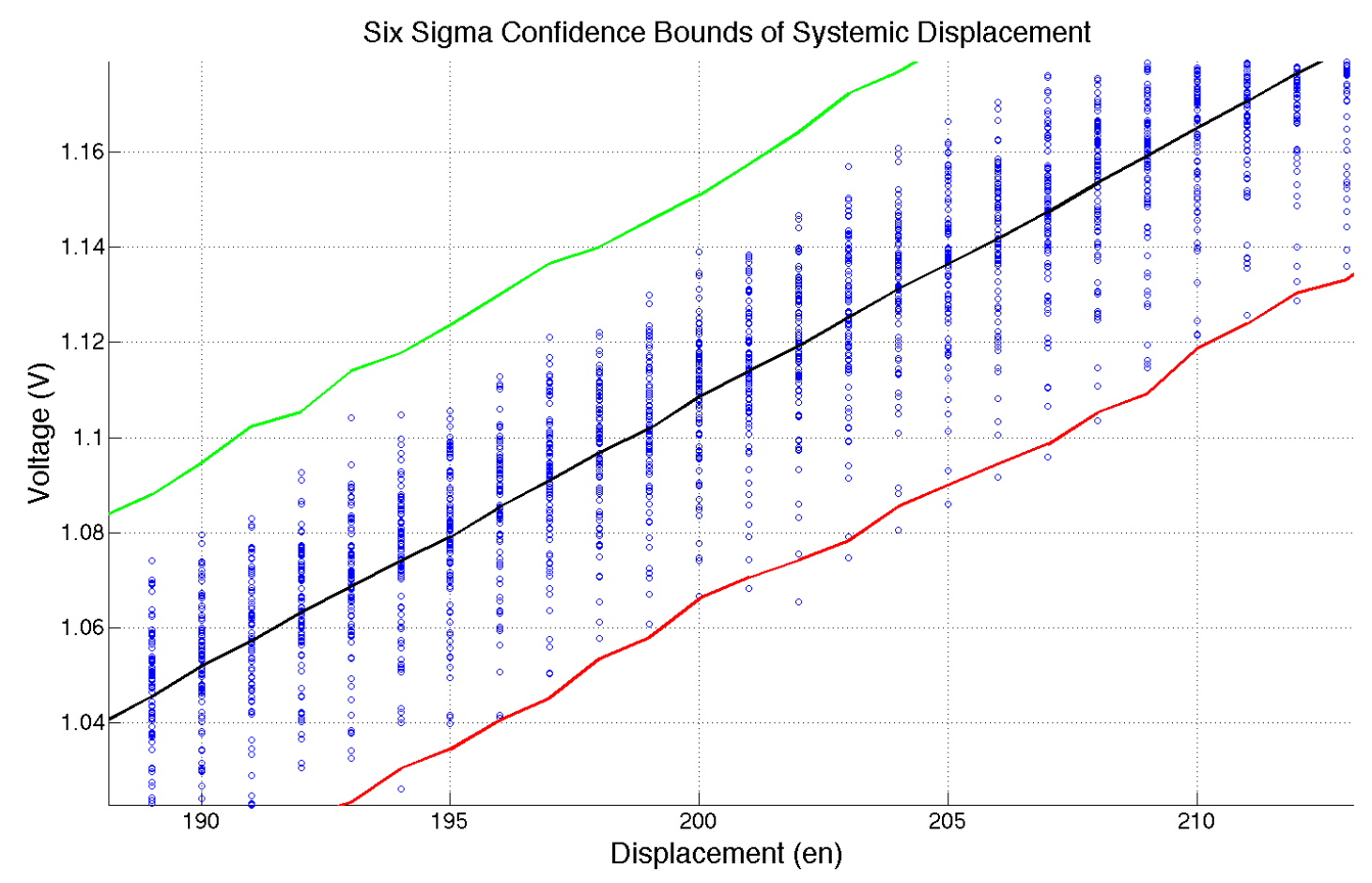

Figure 25: Systemic displacement at middle range of displacement. The green line represents the +3 sigma upper confidence interval, the red line represents the -3 sigma lower confidence interval, with the black line representing the average. The blue circles represent the individual data points from each test run.

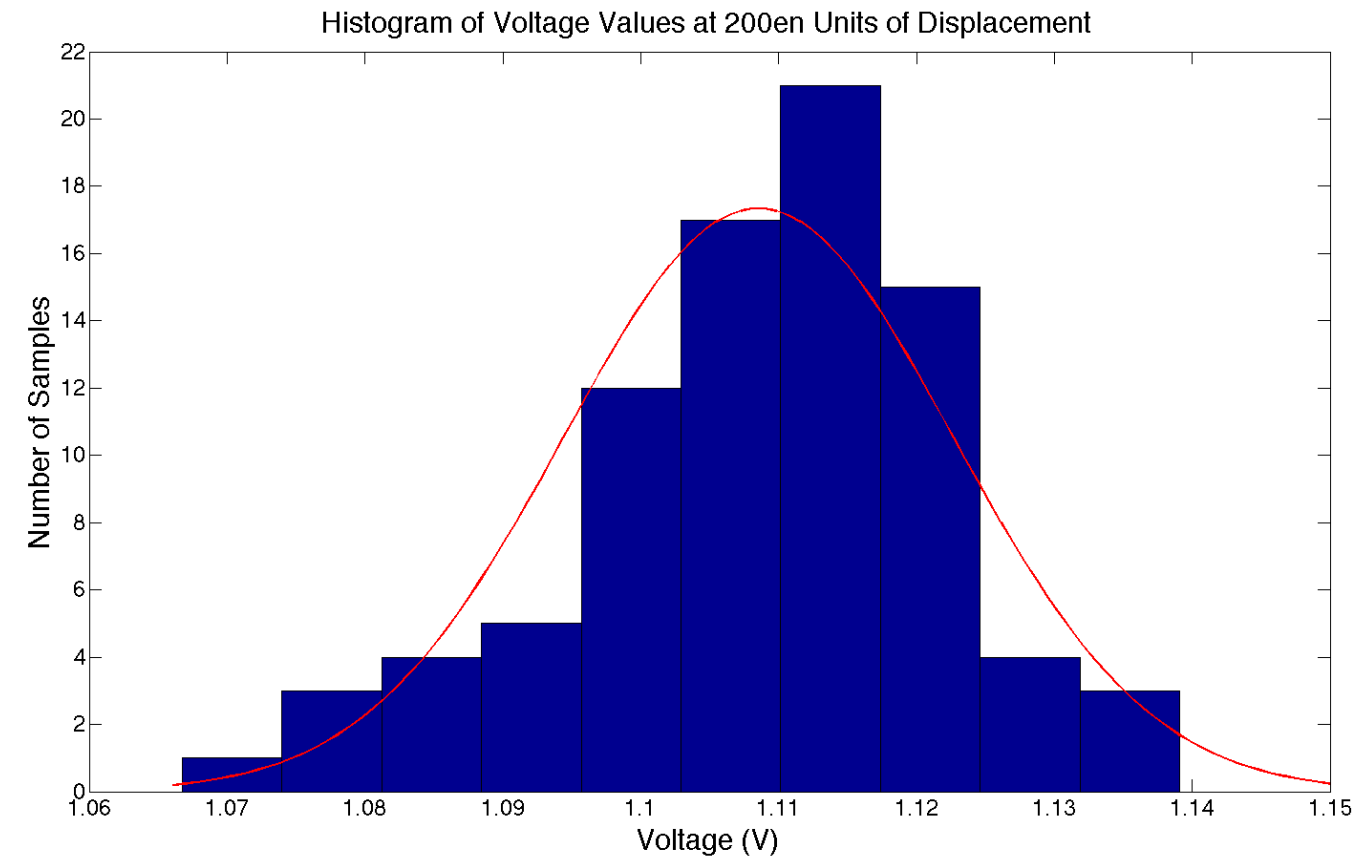

Figure 26: Histogram of voltage at 200en units of displacement. The data appears to be normally distributed. 


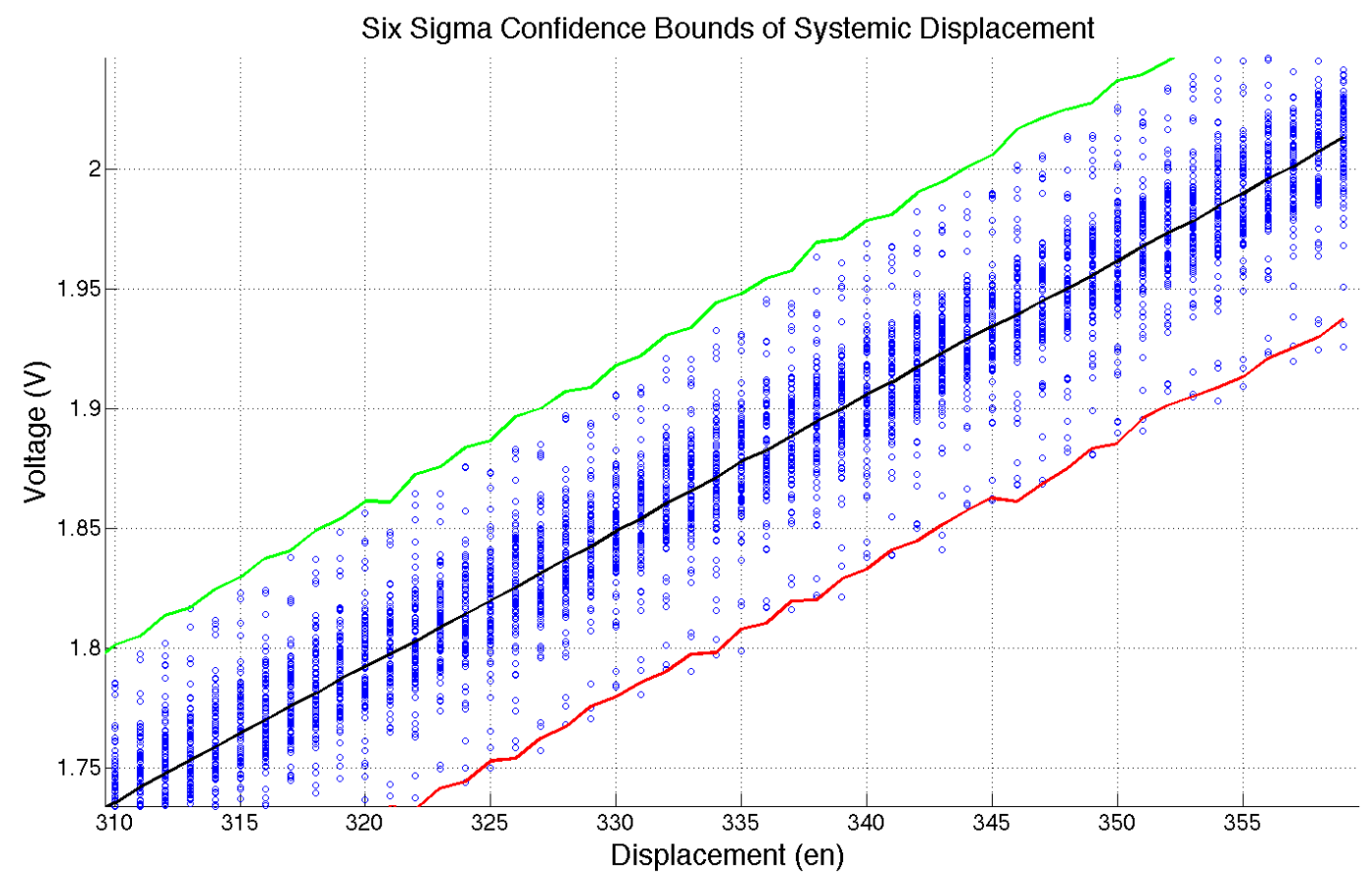

Figure 27: Systemic displacement at high-end range of displacement. The green line represents the +3 sigma upper confidence interval, the red line represents the -3 sigma lower confidence interval, with the black line representing the average. The blue circles represent the individual data points from each test run.

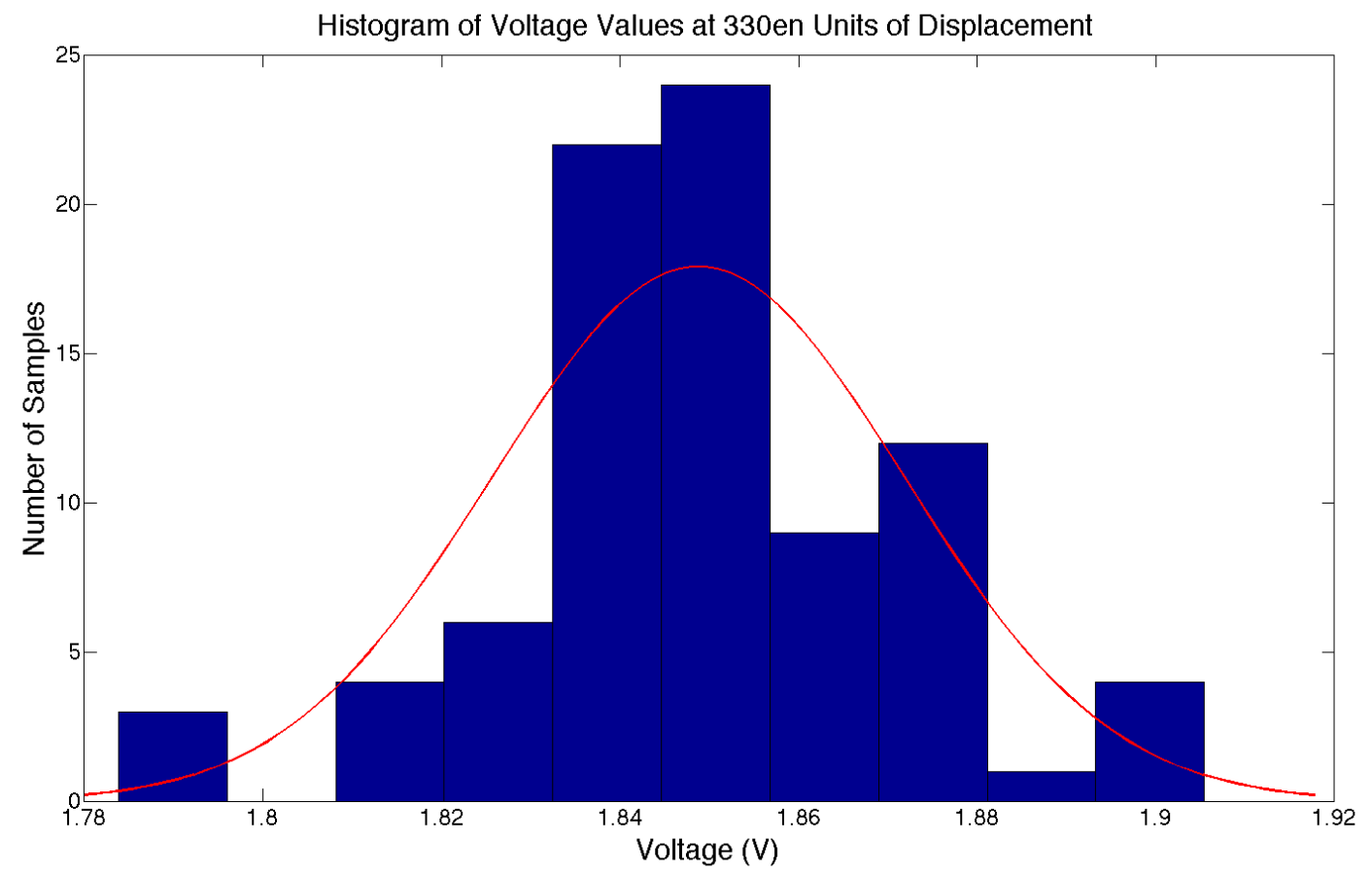

Figure 28: Histogram of voltage at 330en units of displacement. The data appears to be normally distributed. 
From the individual confidence interval taken at each displacement unit, a slope was created using a linear fit. The slopes can be better understood as an inverse, with units of en/ $\mathrm{V}$, meaning that on average, the system will displace an average of 176.75 en units per $1 \mathrm{~V}$ ouput of force. Using the z-axis calibration, the encoder units can be converted into microns using a value of 28.281 en/um (Table 4).

Table 4: Results of systemic displacement testing.

\begin{tabular}{|l|l|l|l|}
\hline & Slope (V/en) & Slope (en/V) & Slope (um/V) \\
\hline +3 Sigma (High End) & $5.8298 \mathrm{E}-3$ & 171.53 & 6.065 \\
\hline Average & $5.6577 \mathrm{E}-3$ & 176.75 & 6.249 \\
\hline -3 Sigma (Low End) & $5.4856 \mathrm{E}-3$ & 182.29 & 6.446 \\
\hline
\end{tabular}

Using the data from Table 4, it is possible to find the upper limit of stiffness that is resolvable in a test sample. Once the stiffness of the sample approached the lower limit of the transducer stiffness, it is no longer possible to differentiate between displacement produced from the sample or from the transducer. Processing the future data with the average slope of the systemic displacement, once the equivalent spring constant approaches the lower limit of the systemic displacement stiffness, the sample stiffness will no longer be resolvable. The equivalent spring constant of both the systemic displacement and sample can be found (Eq. 8)

$$
\frac{1}{K_{\text {sd }}}+\frac{1}{K_{\text {sample }}}=\frac{1}{K_{\text {system }}}
$$

Eq. 8

Taking the inverse of the equation and plugging in the average systemic displacement as well as the lower limit of systemic displacement stiffness for the equivalent spring constant (Eq. 9). 


$$
6.249 \frac{u m}{V}+K_{\text {sample }}^{-1}=6.446 \frac{u m}{V}
$$

Eq. 9

Solving the equation, the maximum theoretical stiffness of a sample can be found to be $5.07 \mathrm{~V} / \mathrm{um}$.

\subsection{TRANSDUCER FORCE TRANSFER FUNCTION}

The raw voltage output from the Keithley 2400 voltmeter must be translated into force to process the data from the MFDS. To accomplish the force calibration, the transducer was lowered onto an Adams Equipment PGW 153e analytical balance with a resolution of $1 \mathrm{mg}$. As the transducer displacement increases, voltage data from the transducer and force data from the analytical balance is collected. The relationship obtained from the force and voltage data allows the raw output voltage from the transducer to be translated into a force measurement.

The transducer was lowered onto the PGW 153e analytical balance 3 times (with 1en displacement steps and a time delay of $1000 \mathrm{~ms}$ between samples), to a force of 150 milligrams (Figure 29). The analytical balance (1 $\mathrm{mg}$ resolution) used in testing was not as sensitive as the transducer (sub-mg range). Thus one of the largest potential sources of error in the MFDS is the transfer function, converting voltage to force. With the combination of low accuracy, and high vibrations in the Microfabrication lab, the data from each of the 3 test runs was combined to produce a linear regression used as the transfer function (Figure 30). The residuals of the linear regression were plotted to ensure the regression was a good approximation of the data (Figure 31). 


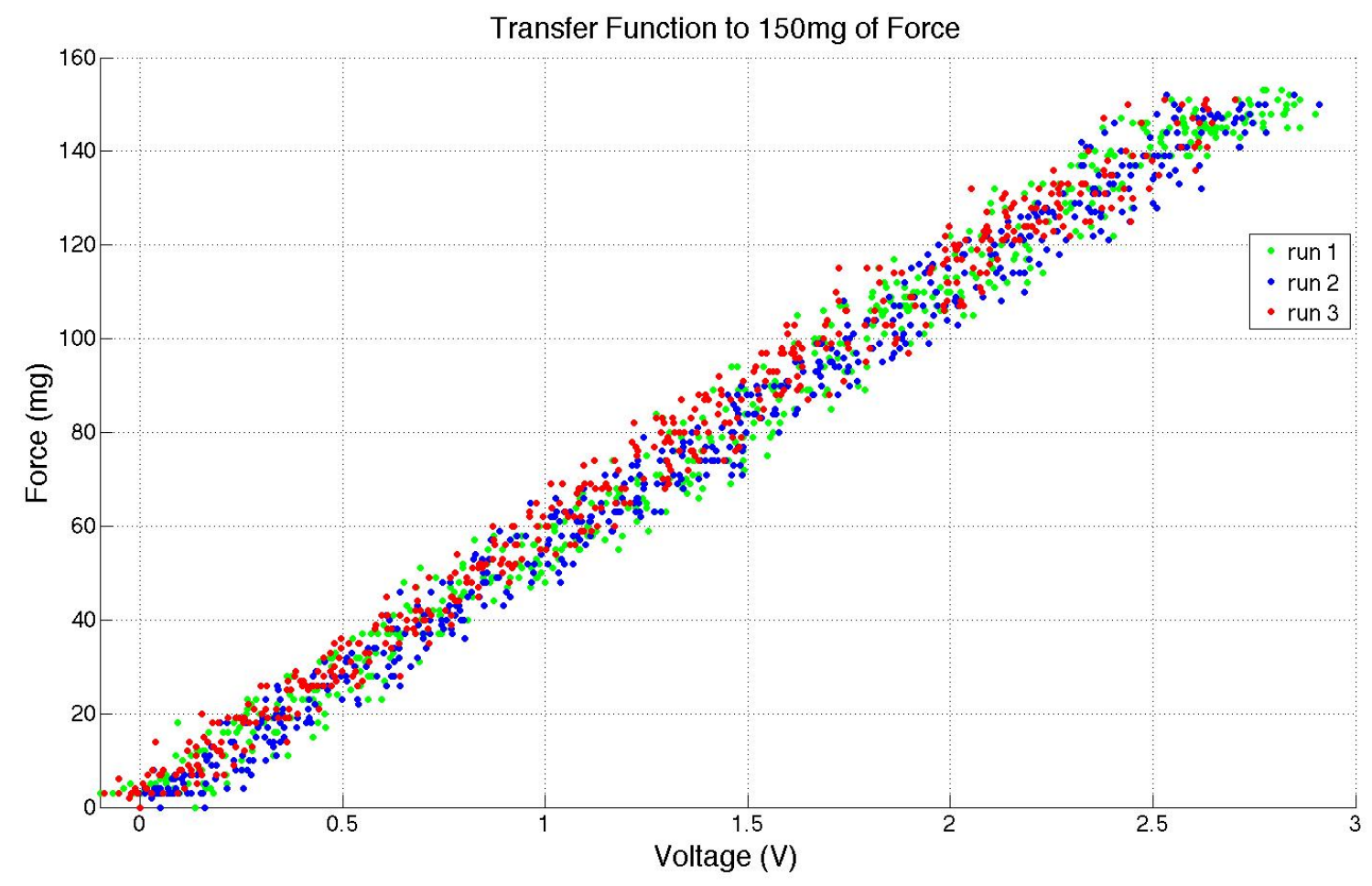

Figure 29: Transfer function from 0 to $150 \mathrm{mg}$ of force. The test was run 3 times to collect more data on the transfer function.

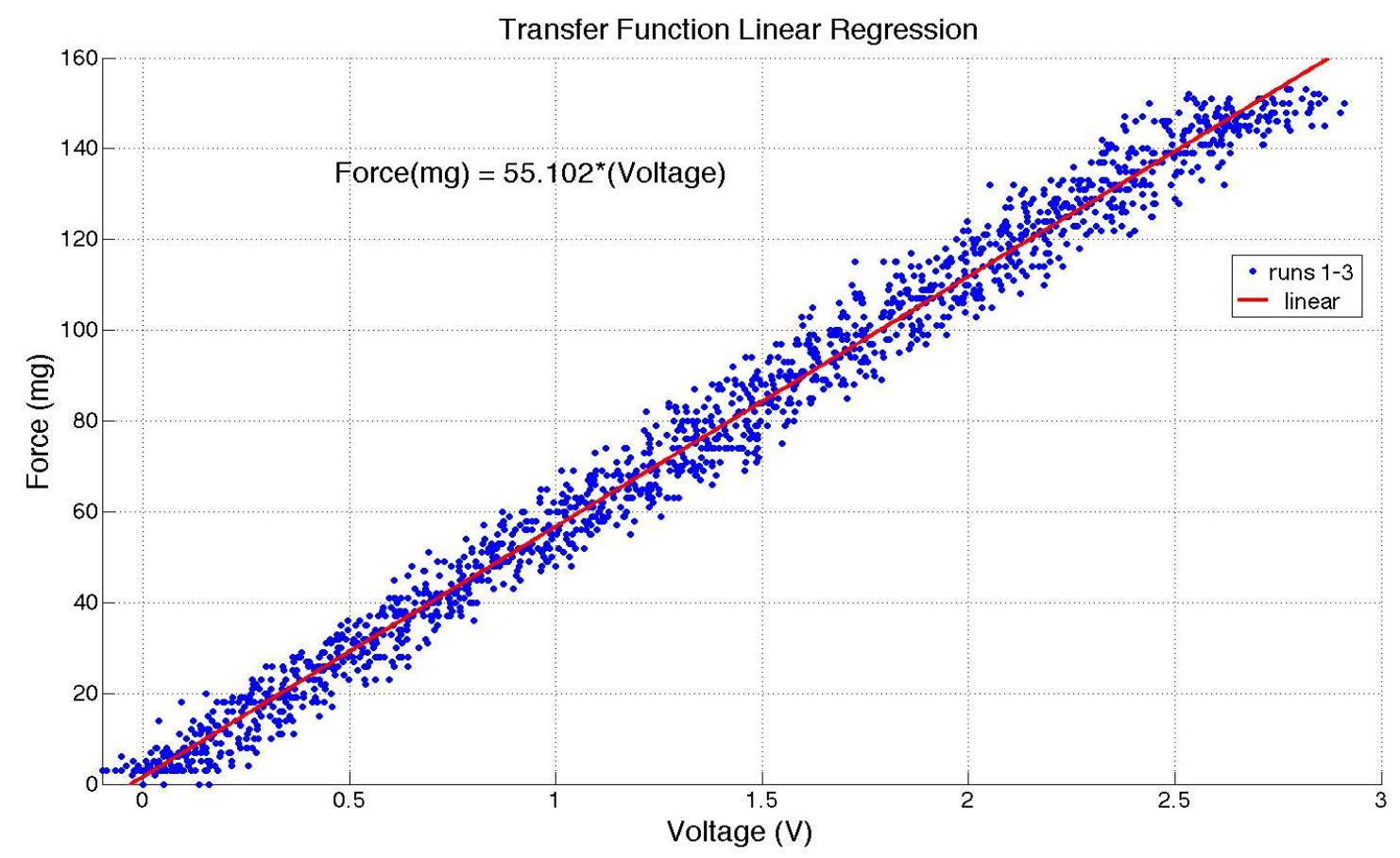

Figure 30: Linear regression produced from 3 test runs of the transducer transfer function. 


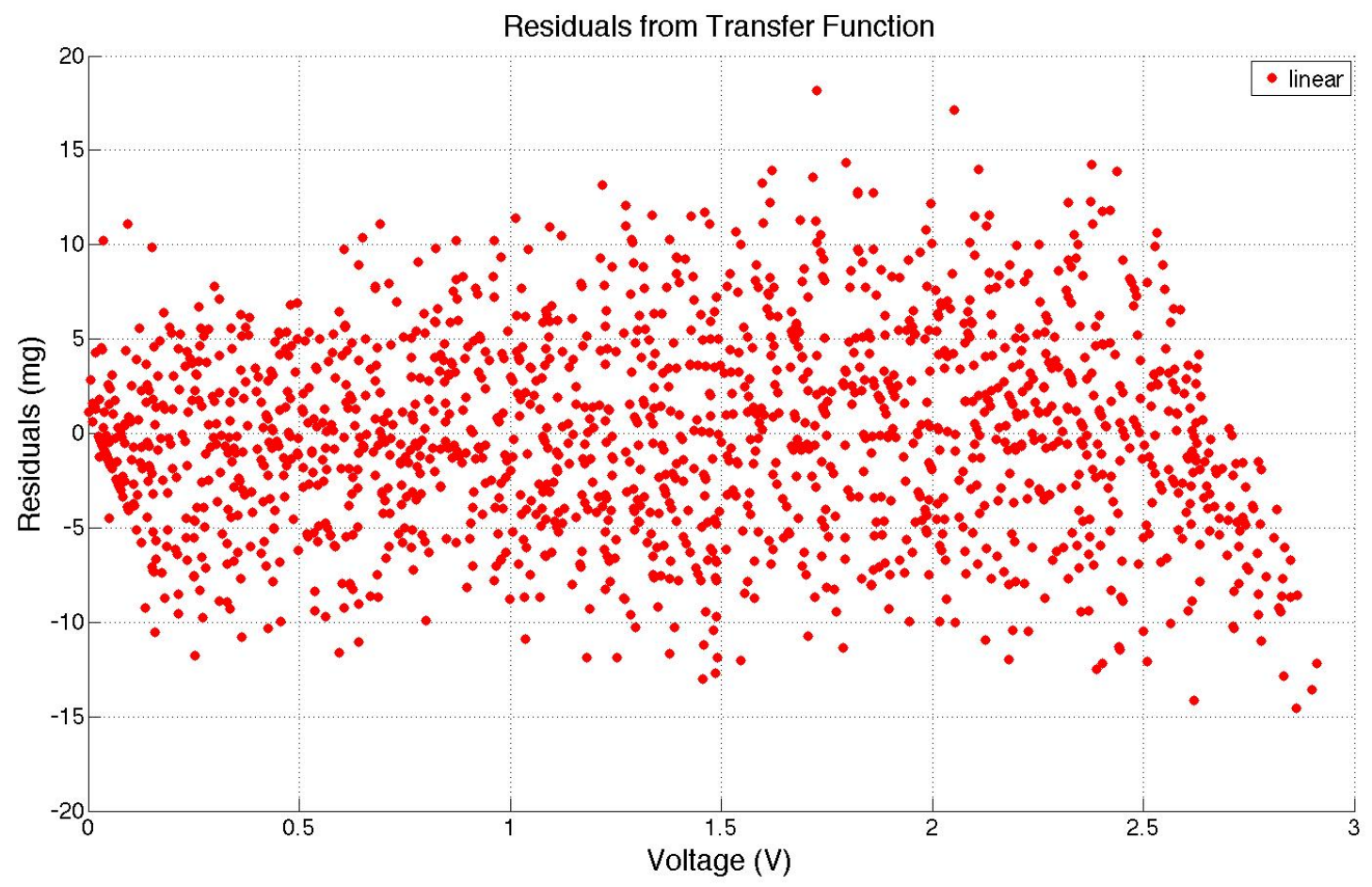

Figure 31: Plot of residuals from transducer transfer function showing that the individual data points are normally distributed about the linear regression (although the residuals are large due to vibrations and the accuracy of the analytical balance).

As is visible in the residuals, the force per given voltage can vary as much as $\pm 15 \mathrm{mg}$ due to the high levels of vibration in the lab and inaccuracies with the analytical balance. Due to the high level of variation present during transfer function testing (but not visible during device testing), it is unreasonable to assign error bars to the final data based on the precision of the transfer function. As such, further testing on the transfer function is necessary to achieve a higher machine precision in the future. Taking the average transfer function equation from Figure 30 , one volt produced from the transducer is equivalent to $55.102 \mathrm{mg}$ of force. Using the slopes from Table 4, the slopes from the systemic displacement can be converted into more concrete units of mg/um (Table 5). 
Table 5: Transfer function applied to systemic displacement slopes.

\begin{tabular}{|l|l|l|}
\hline & Slope (um/V) & Slope (mg/um) \\
\hline +3 Sigma (High End) & 6.065 & 9.084 \\
\hline Average & 6.249 & 8.816 \\
\hline -3 Sigma (Low End) & 6.446 & 8.548 \\
\hline
\end{tabular}

\subsection{CYCLIC LOADING CHARACTERIZATION}

An issue observed during calibration was drift between samples. Over time however, the drift subsided to negligible levels. To characterize the drift, the transducer was brought into contact with a glass microscope slide until the transducer registered approximately 2 volts $(\sim 100 \mathrm{mg})$. Next, data was collected once per second for approximately 25 minutes, finally the transducer was lifted off the glass microscope slide, left for 25 minutes, then the test was repeated. After 3 iterations, the data was collected and plotted (Figure 32). 


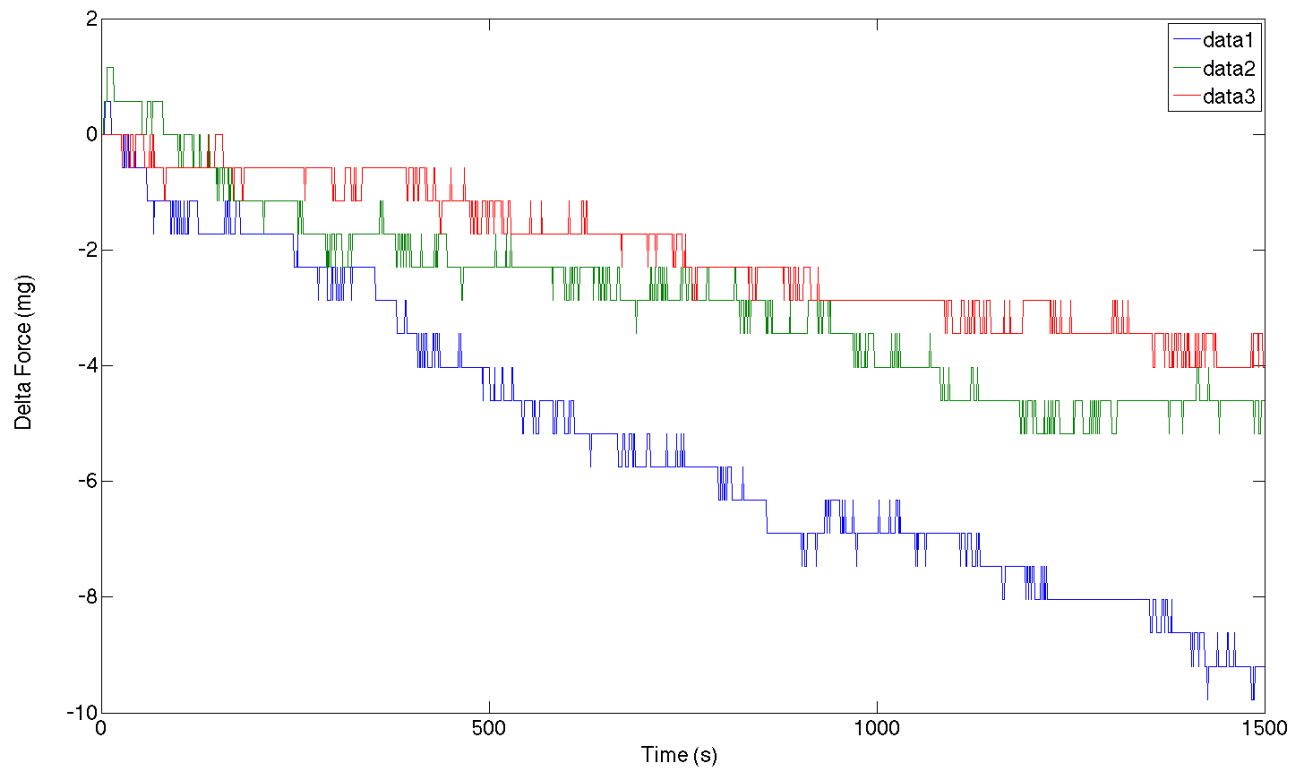

Figure 32: Cyclic loading of transducer to characterize the warm up time of the system.

The first sample (data 1) drops 2 milligrams of force in the first 60 seconds, suggesting that data collected when the system is first turned on could be skewed beyond the confidence of the force characterization previously performed. By the third sample (data 3 ) the change in force does not exceed $+/-0.5$ milligrams until after 7 minutes, and stays at zero for 10 seconds at the beginning of the test, allowing for accurate transducer measurements. From this data, the system should be allowed to warm up for at least 1.5 to 2 hours prior to testing MEMS devices. 


\section{DiAPHRAgM DESIGN}

\subsection{PROCESS SUMMARY}

A n-type Silicon-on-Insulator (SOI) wafer with a 10um thick device layer, 400um thick handle, and 6000 angstroms of buried oxide was selected as the device wafer to construct silicon diaphragms. The device layer of the SOI process wafers was used as the structural diaphragm material, making the diaphragms 10 um thick. The wafers were first thermally oxidized to create a mask for dual doped TMAH etching. Next, positive photoresist was applied and patterned. A BOE etch was used to pattern the diaphragm windows into the oxide layer. The positive photoresist was removed, and then the wafer was etched in a dual doped TMAH solution to remove the silicon of the handle side behind the device layer of the SOI process wafers. The buried oxide of the SOI process wafers served as an etch stop to prevent etching of the device layer of the SOI process wafers. Finally a BOE etch was used to remove the buried oxide as well as the thermal oxide, leaving silicon diaphragms with a side length of 4,5 , and $7 \mathrm{~mm}$.

\subsection{DIAPHRAGM MATERIAL SELECTION}

Several other diaphragm materials were considered, including SU-8, platted copper, and aluminum.

An SU-8 diaphragm would be composed of a silicon wafer with the diaphragm structure constructed from SU-8 polymer. Diaphragms of this nature would need to be constructed much thicker than metal or silicon diaphragms due to the low Young's Modulus of SU-8 $(4.02 \mathrm{Gpa})^{14}$ and diaphragms of this nature would not have many potential applications in the Microfabrication Lab. 
A second option that was explored was the use of copper platting to create a diaphragm. The largest hurdle of this method was the lack of processing equipment available to perform copper platting. In addition, creating a uniform platting thickness was a concern.

An aluminum diaphragm was an option, as the tools to sputter aluminum were available in the lab. In addition, the dual doped TMAH etch selected was supposedly passivizing to aluminum ${ }^{15}$. Tests were performed with a (100) n-type wafer with the same diaphragm pattern as the final process wafers (Figure 45). A 1 um thick layer of aluminum was sputtered on the back side of the oxide mask windows, creating aluminum diaphragms 1 um thick once the dual doped TMAH etch was completed. The test wafers were etched in a dual doped TMAH solution (Figure 33 and Figure 34) but the sputtered aluminum layer was clearly not passivated in the etch chamber. In addition, sputtered metals typically have high internal stresses ${ }^{5}$ leaving the possibility that the final diaphragms would have a large amount of warpage, making them unusable. 


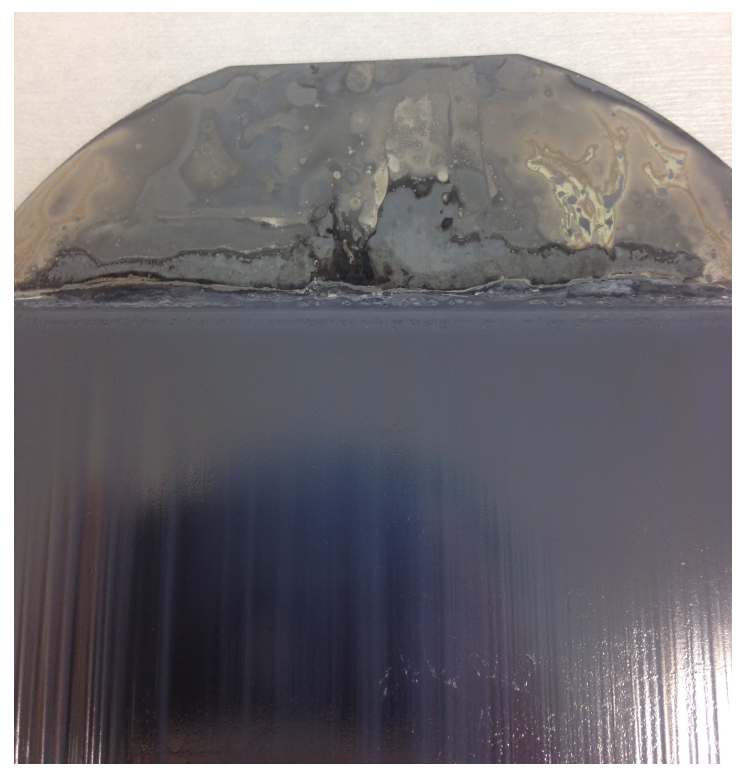

Figure 33: Wafer with aluminum layer sputtered on the backside of the wafer to create $1 \mathrm{um}$ aluminum diaphragms. The dual doped TMAH solution has etched through the aluminum layer into the silicon, destroying the diaphragm material.

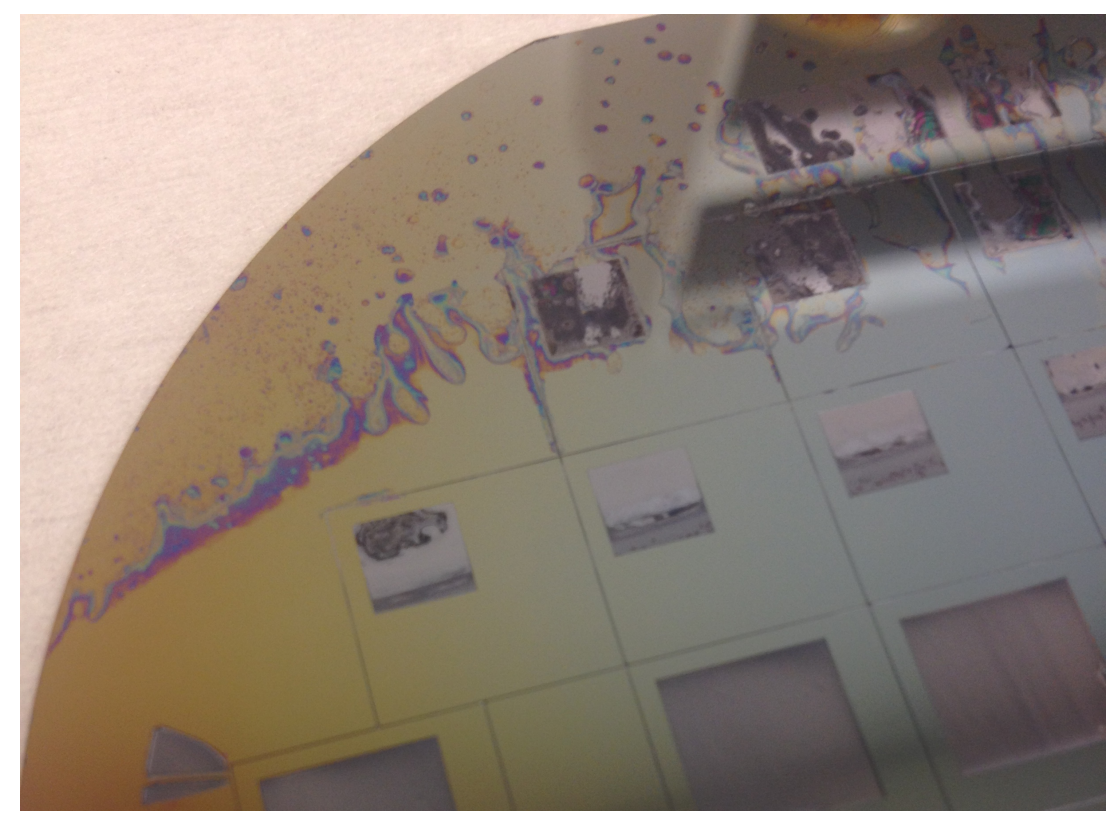

Figure 34: Wafer with aluminum layer sputtered on the backside of the wafer creating $1 \mathrm{um}$ aluminum diaphragms. The middle row of the diaphragms shown coincided with the etchant level in the etching chamber. 
Due to the various issues with other wafer/etchant combinations, a SOI wafer was chosen as the process wafers (Figure 35). The thickness of the diaphragms would be approximately the thickness of the device layer of the SOI process wafer, while the buried oxide would provide ample etch stop capabilities, reducing the time sensitivity of the dual doped TMAH etch.

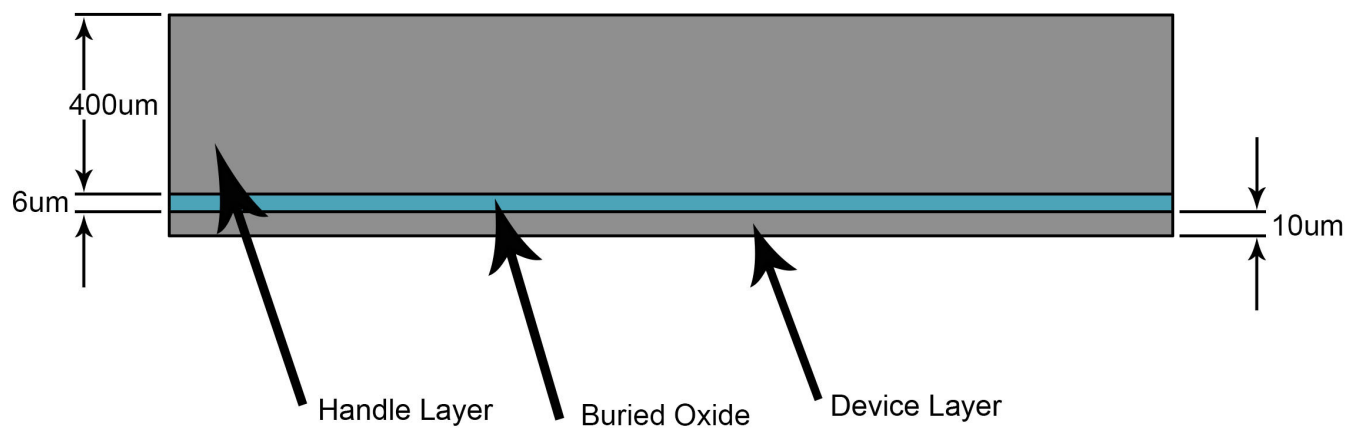

Figure 35: As received SOI process wafer with 400um handle side, 10um device side, and 6um buried oxide layer.

\subsection{LITHOGRAPHY MASK SIZE MODELING}

With a nominal diaphragm thickness of 10um, the side length size of the diaphragms was determined. To reduce cost, a diaphragm lithography mask already created in the lab (Figure 36) was modeled to assess the feasibility recycling the mask in place of ordering a new lithography mask. The lithography mask contained diaphragm mask windows of side length $4.6 \mathrm{~mm}, 5.6 \mathrm{~mm}$, and $7.6 \mathrm{~mm}$ to create diaphragms of side length $4 \mathrm{~mm}, 5 \mathrm{~mm}$, and $7 \mathrm{~mm}$ when considering the anisotropic etch which will narrow each side by about 300 um due to the $54.7^{\circ 23}$ anisotropic etch. 


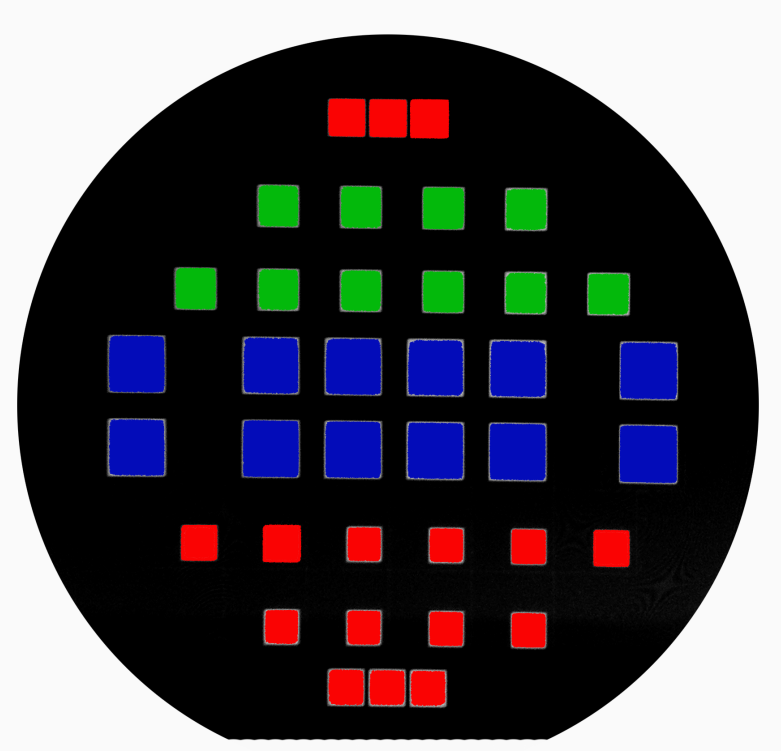

Figure 36: Location of different size diaphragms on photolithography mask used to create diaphragms. Red squares represent $4 \mathrm{~mm}$ diaphragms, green squares represent $5 \mathrm{~mm}$ diaphragms, and blue squares represent $7 \mathrm{~mm}$ diaphragms.

Three important equations were used to model the diaphragms, the maximum stress at the center of the diaphragm, the maximum stress at the middle of each edge, and the maximum displacement at the center of the diaphragm. ${ }^{16}$ The boundary conditions chosen for the diaphragms was clamped edge (approximating the fixed edges of the diaphragm when it met the edge of the sidewall). The loading condition was a concentrated load at the center of the diaphragm, with the probe tip radius ( $\sim 10$ um as measured through optical microscopy) small enough to approximate a point force while not being large enough to not create additional stress around the loading point. Some assumptions of these equations is that the diaphragm thickness is less than $10 \%$ of the side length of the diaphragm (actual: $0.25 \%$ ) and the deflection is less than half the plate thickness. ${ }^{16}$ 
Maximum displacement at center of diaphragm:

$$
y_{m}=k_{1} \frac{P b^{2}}{E t^{3}}
$$

Eq. $10^{16}$

Maximum stress at the center of the diaphragm:

$$
\sigma_{m c}=\frac{1.5 P}{\pi t^{2}}\left((1+v) \ln \left(\frac{2 b}{\pi e}\right)+k_{3}\right)
$$

Eq. $11^{16}$

Maximum stress middle of edge:

$$
\sigma_{m}=k_{2} \frac{P}{t^{2}}
$$

Eq. $12^{16}$

Table 6: Diaphragm $\mathrm{K}$ values for $\mathrm{A} / \mathrm{B}$ ratio of 1:1(Square Diaphragms) ${ }^{16}$

\begin{tabular}{|l|l|}
\hline A/B Ratio & $1: 1$ \\
\hline$k_{1}$ & 0.061 \\
\hline$k_{2}$ & 0.754 \\
\hline$k_{3}$ & -0.238 \\
\hline
\end{tabular}

Values (Table 7) were inserted into Eq. 10 from 0 to 10 um of displacement for $4 \mathrm{~mm}, 5 \mathrm{~mm}$, and $7 \mathrm{~mm}$ diaphragms (Figure 37 ). The Young's modulus was chosen for the (110) plane because it was perpendicular to the axis of testing. 
Table 7: Values used for diaphragm modeling.

\begin{tabular}{|l|l|}
\hline Variable & Value \\
\hline$t=$ thickness & $10 \mathrm{um}$ \\
\hline$b=$ side length & $4 \mathrm{~mm}, 5 \mathrm{~mm}, 7 \mathrm{~mm}$ \\
\hline$E=$ Youngs Modulus & $170 \mathrm{GPa}^{1 /}$ \\
\hline$e=$ Tip radius & $5 \mathrm{um}$ \\
\hline$v=$ Poisson's ratio & $0.23^{1 /}$ \\
\hline
\end{tabular}

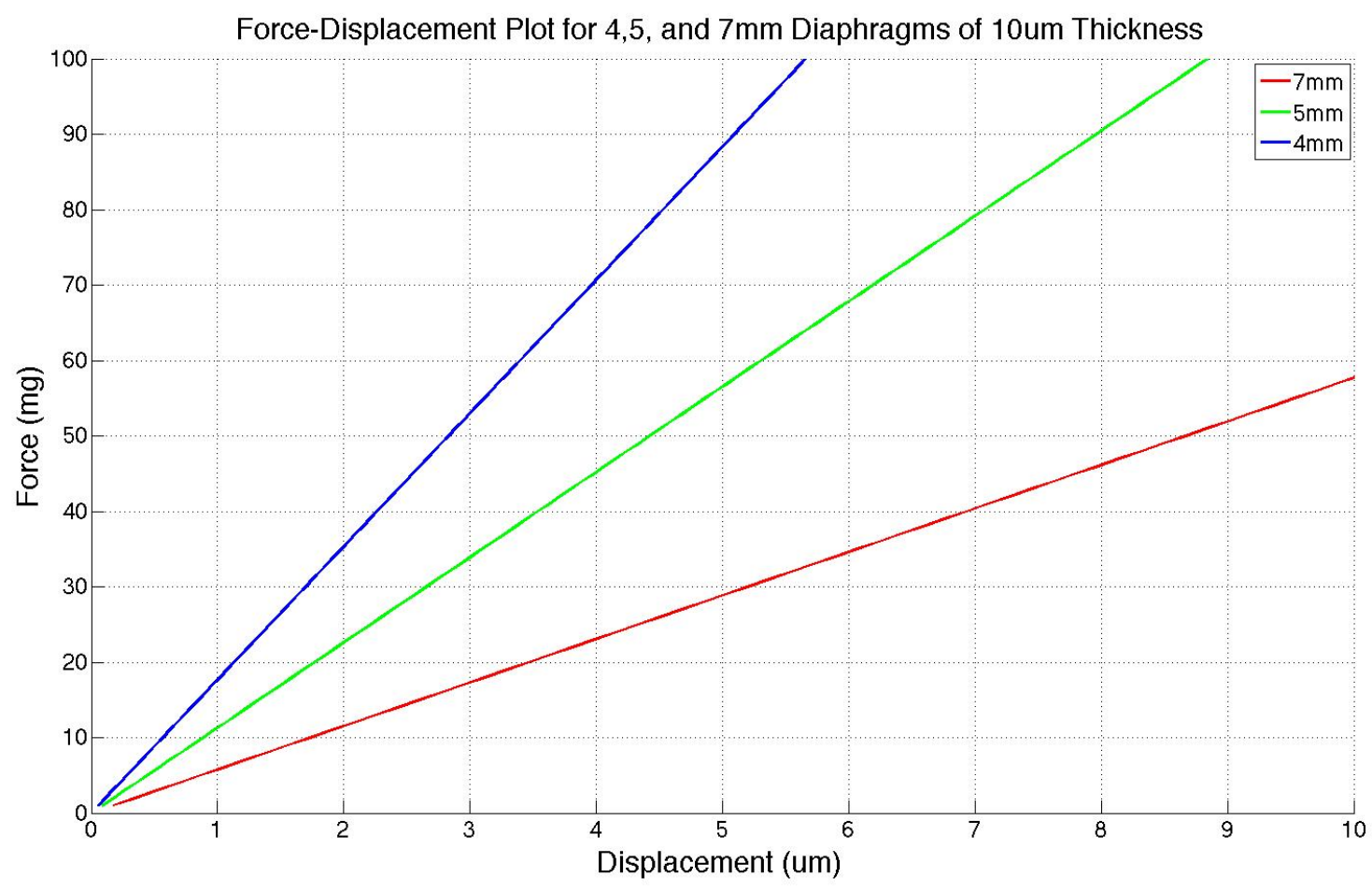

Figure 37: Force-displacement plot of 4, 5, and $7 \mathrm{~mm}$ diaphragms of $10 \mathrm{um}$ thickness from theoretical linear model. 
From Eq. 10, linear spring constants for each diaphragm could be determined (Table 8) which were then compared to the lower confidence interval of the systemic displacement of $8.548 \mathrm{mg} / \mathrm{um}$. All 3 diaphragm sizes are theoretically resolvable within the linear range of diaphragm displacement.

Table 8: Equivalent system spring constant produced from different sample sizes.

\begin{tabular}{|l|l|l|}
\hline $\begin{array}{l}\text { Diaphragm } \\
\text { Size }\end{array}$ & $\begin{array}{l}\text { Spring Constant } \\
\text { Diaphragm }\end{array}$ & $\begin{array}{l}\text { Resultant } \mathbf{K}_{\text {system }} \text { (based on systemic K of } \\
\mathbf{8 . 8 1 6} \mathrm{mg} / \mathrm{um})\end{array}$ \\
\hline $4 \mathrm{~mm}$ & $17.669 \mathrm{mg} / \mathrm{um}$ & $5.882 \mathrm{mg} / \mathrm{um}$ \\
\hline $5 \mathrm{~mm}$ & $11.308 \mathrm{mg} / \mathrm{um}$ & $4.954 \mathrm{mg} / \mathrm{um}$ \\
\hline $7 \mathrm{~mm}$ & $5.7695 \mathrm{mg} / \mathrm{um}$ & $3.484 \mathrm{mg} / \mathrm{um}$ \\
\hline
\end{tabular}

The maximum stress was modeled at the center and middle of the side length for each diaphragm size. The maximum stress was higher at the center of the diaphragm (due to the relatively small tip radius) for all 3 sizes. The maximum stresses produced were found to be well under the fracture strength for silicon $(4.82 \mathrm{GPa})^{18}$ 


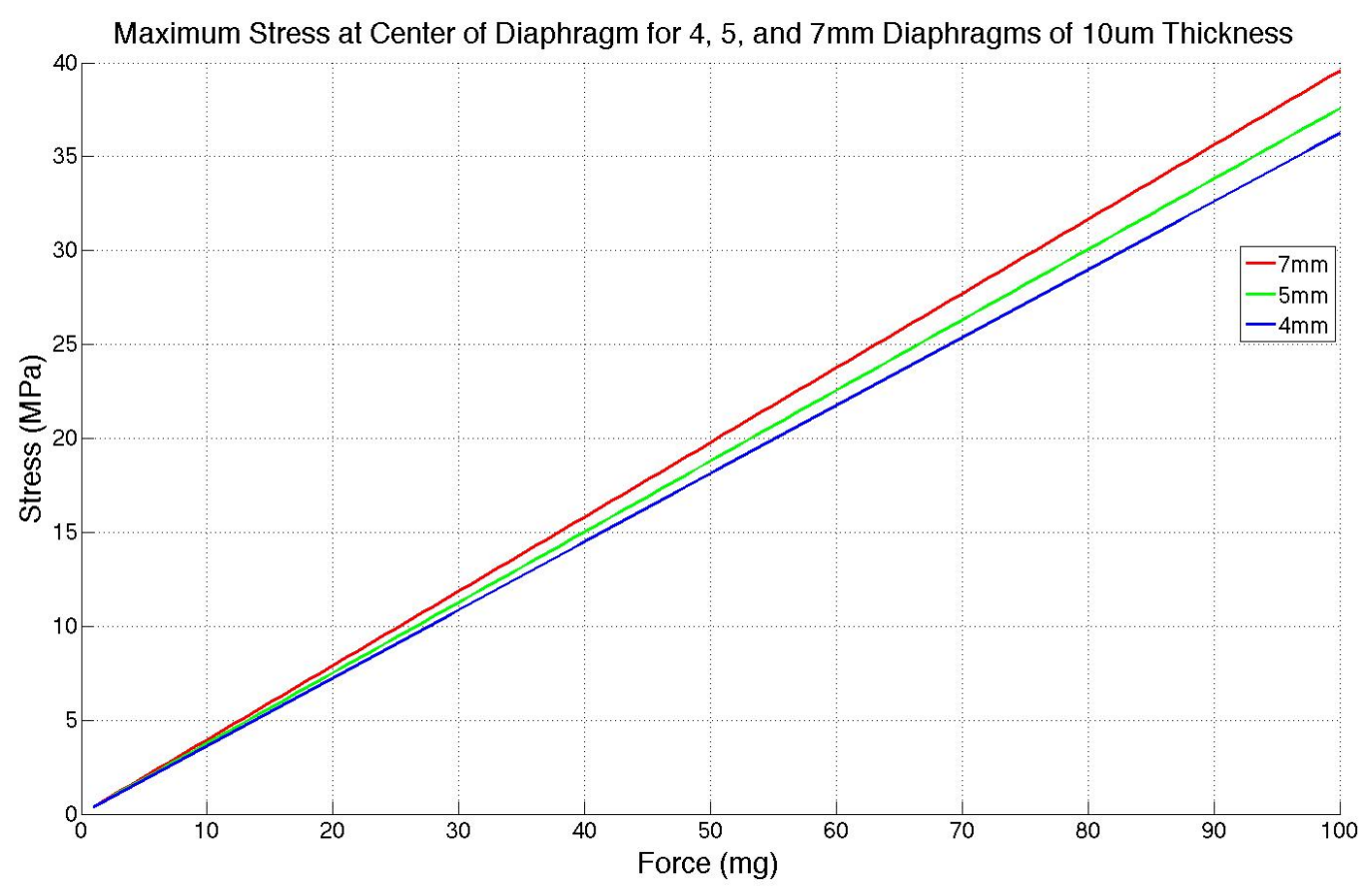

Figure 38: Maximum stress at center of diaphragm for 4,5 , and $7 \mathrm{~mm}$ diaphragms of $10 \mathrm{um}$ thickness.

From the results of the lithography mask size modeling, diaphragms of side length $4 \mathrm{~mm}, 5 \mathrm{~mm}$, and $7 \mathrm{~mm}$ were chosen to be appropriate as test structures. Later testing (Section 6) revealed the discrepancies between the calculated and average observed values for force and displacement at 1.75 and 3.5um (Table 9). 
Table 9: Comparison of Theoretical and Measured Results at Select Displacement Values.

\begin{tabular}{|l|l|l|}
\hline & \multicolumn{2}{|c|}{ Force at Select Displacement Values } \\
\hline Sample & $\begin{array}{l}\text { 1.75 um (\% change } \\
\text { from theoretical) }\end{array}$ & $\begin{array}{l}\text { 3.5 um (\% change } \\
\text { from theoretical }\end{array}$ \\
\hline Wafer 1, 4mm & $20.49 \mathrm{mg} \mathrm{(33 \% )}$ & $43.30 \mathrm{mg} \mathrm{(30 \% )}$ \\
\hline Wafer 1, 5mm & $15.75 \mathrm{mg} \mathrm{(20 \% )}$ & $33.16 \mathrm{mg} \mathrm{(16 \% )}$ \\
\hline Wafer 1,7mm & $10.78 \mathrm{mg} \mathrm{(7 \% )}$ & $22.04 \mathrm{mg} \mathrm{(9 \% )}$ \\
\hline Wafer 2, 4mm & $26.78 \mathrm{mg} \mathrm{(13 \% )}$ & $56.22 \mathrm{mg} \mathrm{(9 \% )}$ \\
\hline Wafer 2, 5mm & $22.17 \mathrm{mg} \mathrm{(12 \% )}$ & $46.31 \mathrm{mg} \mathrm{(17 \% )}$ \\
\hline Wafer 2, 7mm & $14.82 \mathrm{mg} \mathrm{(46 \% )}$ & $30.19 \mathrm{mg} \mathrm{(49 \% )}$ \\
\hline Theoretical 4mm & $30.92 \mathrm{mg}$ & $61.84 \mathrm{mg}$ \\
\hline Theoretical $\mathbf{5 m m}$ & $19.79 \mathrm{mg}$ & $39.58 \mathrm{mg}$ \\
\hline Theoretical 7mm & $10.09 \mathrm{mg}$ & $20.19 \mathrm{mg}$ \\
\hline
\end{tabular}




\section{DIAPHRAGM FABRICATION}

\subsection{WET THERMAL OXIDATION}

The process design called for approximately 6 hours of etch time, with the dual doped TMAH solution removing roughly 75 angstroms ${ }^{15}$ of silicon dioxide per hour, 450 angstroms of oxide were necessary to protect the silicon from etching. Because the buried oxide on the SOI wafer was 6000 angstroms, the oxide was designed to come close to the buried oxide thickness after etching and processing. A target thickness of 7700 angstroms was chosen so that the post oxide thickness would be within 2000 angstroms of the buried oxide, with ample thickness available if multiple BOE cleanings were necessary before lithography (Figure 39).

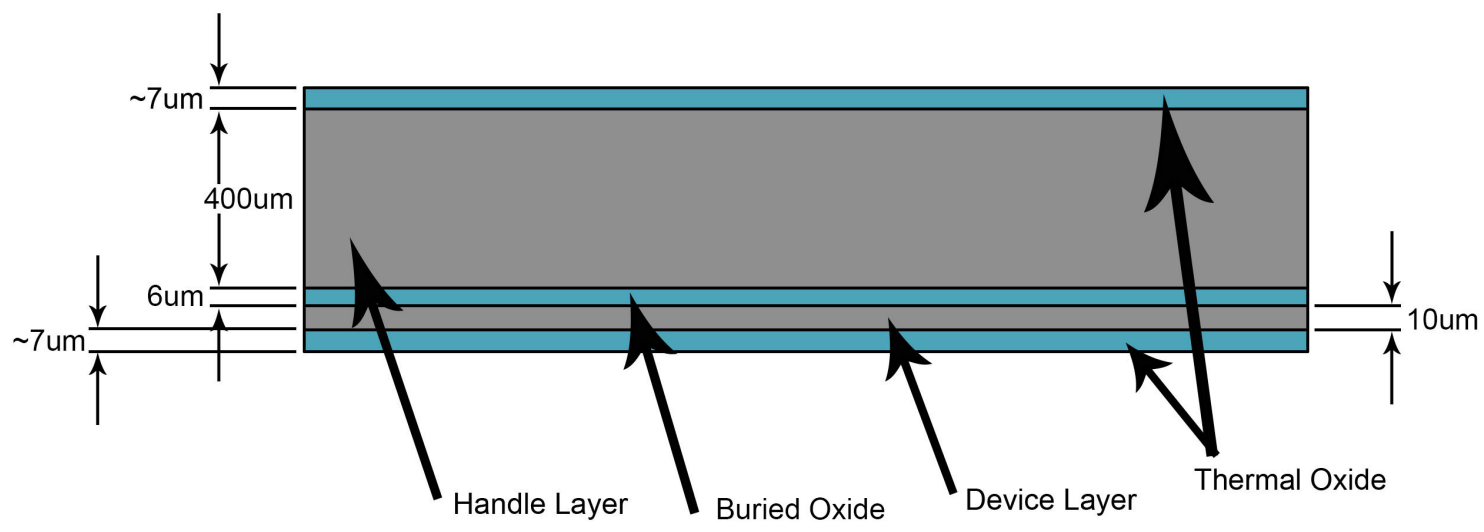

Figure 39: Theoretical result of thermal oxidation processing. 
The Deal Grove model was used to approximate the time needed to grow the film, as the time was sufficiently large, the linear term can be ignored, and the equation for film thickness is:

$$
\frac{x_{0}}{A / 2} \cong\left(\frac{t}{A^{2} / 4 B}\right)^{\frac{1}{2}}
$$

Eq. $13^{19}$

This equation can be further simplified to:

$$
x_{0}^{2} \cong B t
$$

Eq. $14^{19}$

With $\mathrm{x}_{0}$ the film thickness $(.77 \mathrm{um})$, B the parabolic rate coefficient $\left(\mathrm{um}^{2} / \mathrm{hr}\right)$, and $\mathrm{t}$ the time needed (hr). B was determined to be $.305 \mathrm{um}^{2} / \mathrm{hr}$ for a wet oxidation at $1050^{\circ} \mathrm{C}^{5}$. The oxidation time was determined to be 1.94 hours, which was rounded to 2 hours. In the process of thermal oxide growth, some of the silicon of the device layer was consumed. This consumption is proportional to the densities of the substrate and oxide:

$$
X_{s i}=X_{o x} \cdot \frac{N_{o x}}{N_{s i}}
$$

Eq. $15^{20}$

Where $X_{\mathrm{si}}$ is the amount of silicon consumed, $X_{\mathrm{ox}}$ is the thickness of the oxide layer, $\mathrm{N}_{\mathrm{ox}}$ is the molecular density of $\mathrm{SiO}_{2}$, and $\mathrm{N}_{\mathrm{si}}$ is the atomic density of silicon. Inserting these values into Eq. 15:

$$
X_{s i}=X_{o x} \cdot \frac{2.3 e 22 \frac{\text { molecules }}{\mathrm{cm}^{3}}}{5 e 22 \frac{\text { atoms }}{\mathrm{cm}^{3}}}=0.46 X_{o x}=0.46(.77 u m)=0.35 \mathrm{um}
$$

Eq. $16^{20}$ 
As shown in Eq. 16, approximately $350 \mathrm{~nm}$ of the device layer of the SOI wafer should be consumed in the oxidation process, reducing the theoretical thickness to 9.65um.

\subsubsection{PROCEDURE}

The two SOI process wafers along with two n-type "dummy wafers" were cleaned in a 9:1 ratio (sulfuric acid:hydrogen peroxide) Piranha solution at $70^{\circ} \mathrm{C}$ for 10 minutes to remove any organic contaminants. The wafers were subsequently cleaned in a BOE solution for 5 minutes to remove any oxidation from the surface of the wafers. Finally the wafers were spin rinsed and dried. The wafer boat was cleaned in the piranha solution to remove any organic contaminants. The wafers were loaded into the wafer boat, with the "dummy wafers" on the outside of the two SOI process wafers. The "dummy wafers" help to control the flow of gas, creating a more even oxide on the SOI process wafers. The furnace was preheated to $900^{\circ} \mathrm{C}$ (Table 10). Once temperature was reached, ultra-high purity (UHP) nitrogen $\left(\mathrm{N}_{2}\right)$ gas was introduced into the furnace at $6 \mathrm{~L} / \mathrm{min}$. The nitrogen helps to limit the oxide growth as the furnace is heated to the target temperature $\left(1050^{\circ} \mathrm{C}\right)$. With the gas flow on, the wafer boat was loaded into the furnace at approximately $1 \mathrm{~cm} / \mathrm{sec}$, slow enough to prevent thermal shock of the wafer boat or wafers. Once the wafer boat was loaded, the furnace temperature was increased to $1050^{\circ} \mathrm{C}$ with the UHP $\mathrm{N}_{2}$ running to prevent unwanted oxidation. With the $1050^{\circ} \mathrm{C}$ temperature reached, the UHP $\mathrm{N}_{2}$ was switched off. UHP oxygen $\left(\mathrm{O}_{2}\right)$ was first bubbled at $6 \mathrm{~L} / \mathrm{min}$ through a flask of boiling water, and then introduced to the furnace as to add wet water vapor for oxidation. The wet oxidation process was performed for two hours, at which point the UHP $\mathrm{O}_{2}$ was turned off, and the UHP $\mathrm{N}_{2}$ was reintroduced into the furnace to prevent unwanted oxidation as the furnace cooled down. With the UHP $N_{2}$ 
flowing, the furnace was shut off. Once the furnace dropped below $900^{\circ} \mathrm{C}$, the UHP $\mathrm{N}_{2}$ was shut off and the furnace was allowed to cool overnight to room temperature (RT).

Table 10: Oxidation Process Steps

\begin{tabular}{|l|l|l|}
\hline Step & Temperature & Gas (Flow Rate) \\
\hline Preheat & RT $-900^{\circ} \mathrm{C}$ & None \\
\hline Wafers Introduced & $900^{\circ} \mathrm{C}$ & UHP N $_{2}(6 \mathrm{~L} / \mathrm{min})$ \\
\hline Ramp up & $900^{\circ} \mathrm{C}-1050^{\circ} \mathrm{C}$ & $\mathrm{UHP} \mathrm{N}_{2}(6 \mathrm{~L} / \mathrm{min})$ \\
\hline Oxidation (2 hours) & $1050^{\circ} \mathrm{C}$ & Wet UHP O $\mathrm{O}_{2}(6 \mathrm{~L} / \mathrm{min})$ \\
\hline Cool Down & $1050^{\circ} \mathrm{C}-900^{\circ} \mathrm{C}$ & UHP N ${ }_{2}(6 \mathrm{~L} / \mathrm{min})$ \\
\hline Cool Down & $900^{\circ} \mathrm{C}-\mathrm{RT}$ & None \\
\hline
\end{tabular}

\subsubsection{RESULTS}

Once the wafers were cooled and removed from the furnace, a Filmetrics F-20 film thickness measurement system was used to measure the thickness of the grown oxide. The thickness of the handle side oxide thickness of the SOI process wafers was measured at various locations (Figure 40) to ensure the thickness would be sufficient for the dual doped TMAH etch (Table 11). The device side of the SOI process wafers was measured to be within 100 angstroms of the handle side of each wafer. 


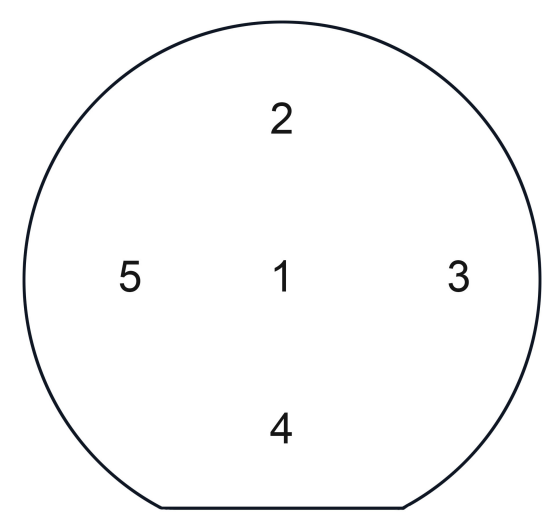

Figure 40: Location of oxide growth measurements on handle side of SOI process wafers.

Table 11: Thickness of oxide grown on SOI process wafers.

\begin{tabular}{|l|l|l|}
\hline Location & SOI Process Wafer \#1 & SOI Process Wafer \#2 \\
\hline 1 & $7150 \AA$ & $7267 \AA$ \\
\hline 2 & $7295 \AA$ & $7371 \AA$ \\
\hline 3 & $7155 \AA$ & $7368 \AA$ \\
\hline 4 & $7136 \AA$ & $7266 \AA$ \\
\hline 5 & $7253 \AA$ & $7290 \AA$ \\
\hline Average & $7198 \AA$ & $7312 \AA$ \\
\hline
\end{tabular}

The thickness of the two SOI process wafers was sufficient to continue onto the next step of processing, lithography. The wafers would have been reworked had the oxide been thinner or uneven. 


\subsection{LITHOGRAPHY}

The purpose of the lithography process was to create a patterned mask of positive photoresist to act as a mask for a subsequent oxide etch. First the wafer was prepared and the positive photoresist was spin coated onto the wafer (Figure 41). Next the positive photoresist was patterned and developed as to create square windows in the positive photoresist to act as a mask for subsequent oxide etching (Figure 42).

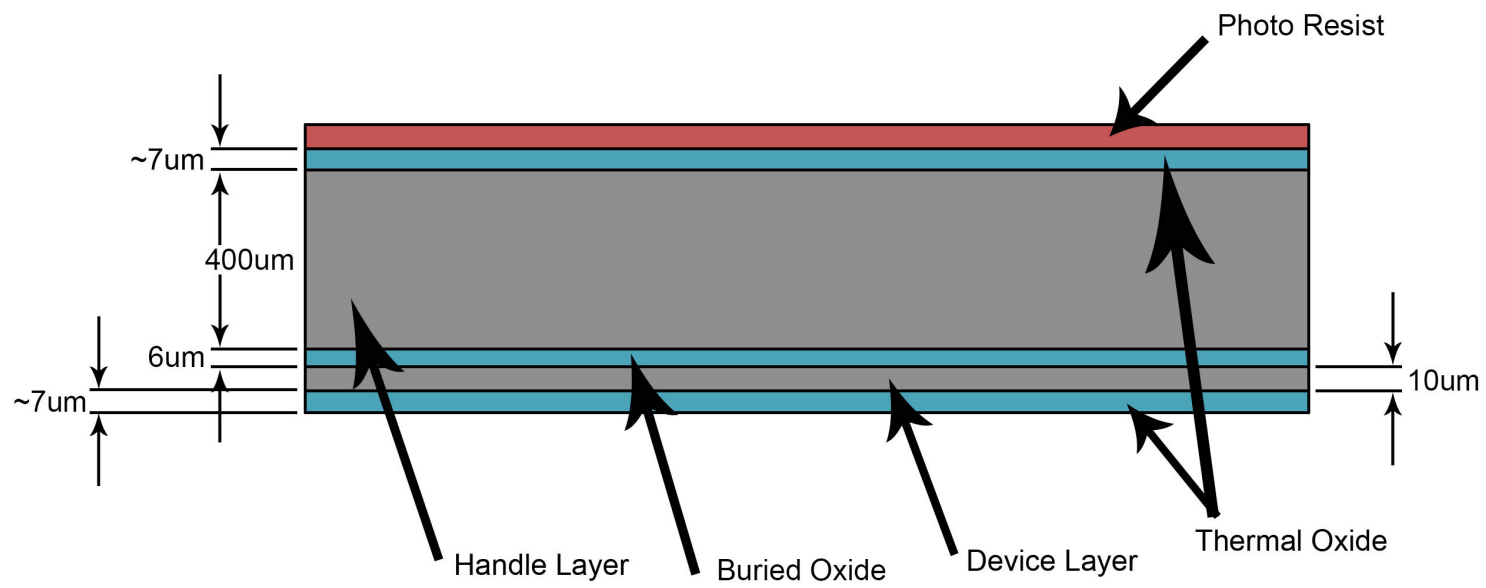

Figure 41: SOI process wafer with positive photoresist applied to the handle side of the wafer.

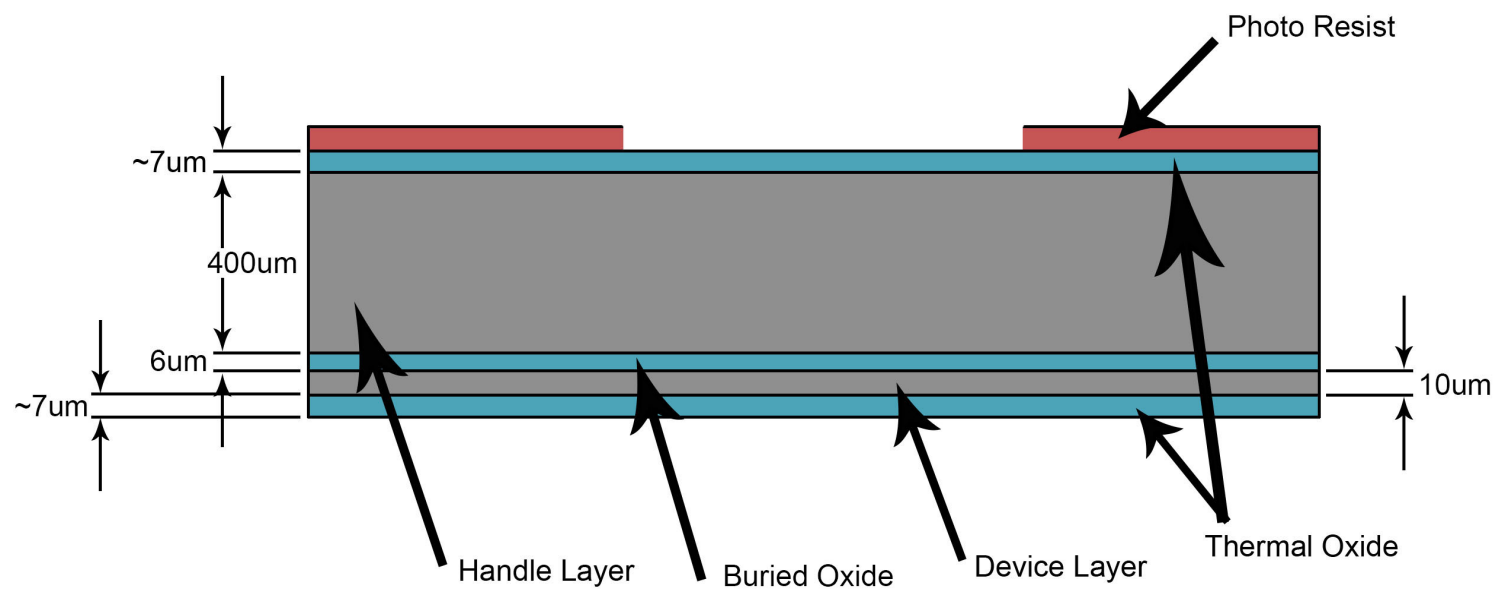

Figure 42: SOI process wafer with positive photoresist applied and patterned on the handle side of the wafer. 


\subsubsection{PROCEDURE}

The one SOI process wafer was first processed to ensure the success of the process before the other SOI process wafer was processed. One SOI process wafer with one "dummy wafer" was cleaned using a 9:1 Piranha solution for 10 minutes at $70^{\circ} \mathrm{C}$. Subsequently the wafers were dipped in BOE for 5 seconds, then spin rinsed and dried. Prior to spin coating, each wafer was placed on a hot plate at $150^{\circ} \mathrm{C}$ for 5 minutes to remove organic solvents left by the cleaning process. The wafer was placed in a Cee 200X spin coater (Figure 43), and centered using a centering chuck. $2.5 \mathrm{~mL}$ of Microchem MCC primer $80 / 20$ was applied to the wafer prior to the spin coating process to promote adhesion between the positive photoresist and the wafer. The spin coating process was initiated, then Microposit s1813 positive photo resist was applied to the wafer in step 4 of the spin coating process (Table 12).

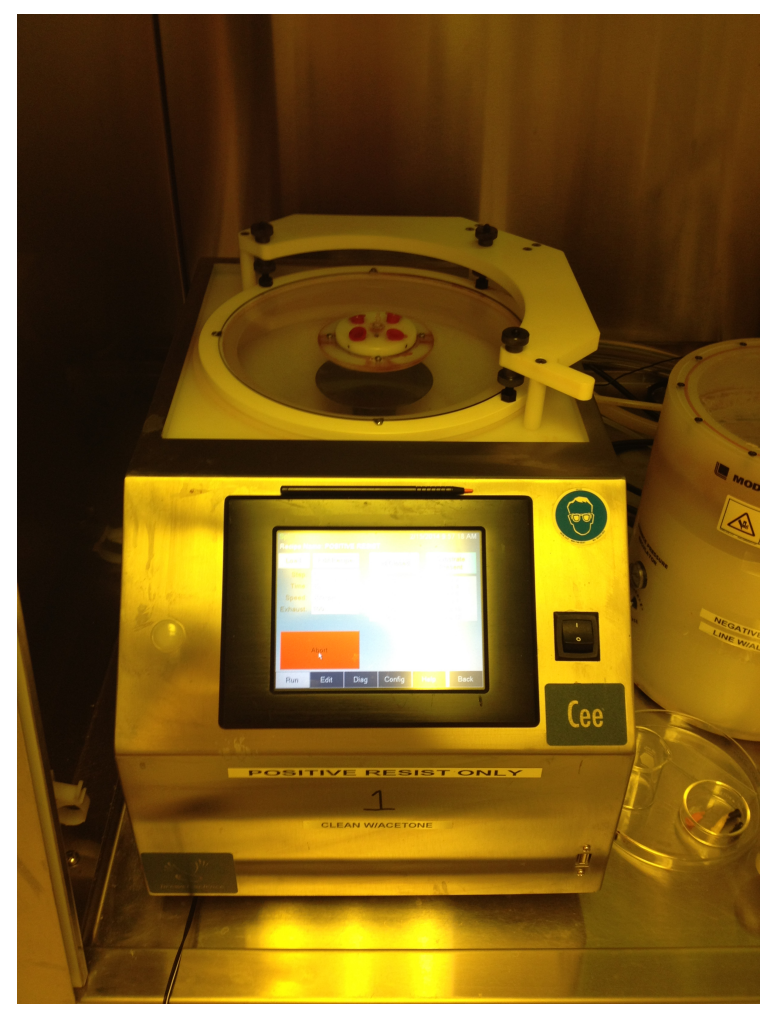

Figure 43: Cee 200X Spin Coater used for the application of positive photo resist. 
Table 12: Spin coating parameters for the application of positive photoresist

\begin{tabular}{|l|l|l|l|l|}
\hline Step \# & RPM & Ramp (RPM/s) & Time (s) & Purpose \\
\hline 1 & 300 & 2040 & 45 & Distribute primer to entire wafer surface \\
\hline 2 & 3000 & 2176 & 30 & Planarize primer to uniform thickness \\
\hline 3 & 100 & 100 & 30 & Slow down wafer for photoresist application \\
\hline 4 & 0 & 0 & 45 & Application of photoresist (wafer stationary) \\
\hline 5 & 500 & 250 & 30 & Distribute photoresist to entire wafer surface \\
\hline 6 & 4000 & 2176 & 30 & Planarize photoresist to uniform thickness \\
\hline 7 & 300 & 500 & 10 & Slow down wafer to stop \\
\hline
\end{tabular}

After the spin coating process, the wafer was carefully removed from the spin coater and placed on a hot plate at $100^{\circ} \mathrm{C}$ (to soft bake prior to the lithography process to drive off solvents and promote adhesion.

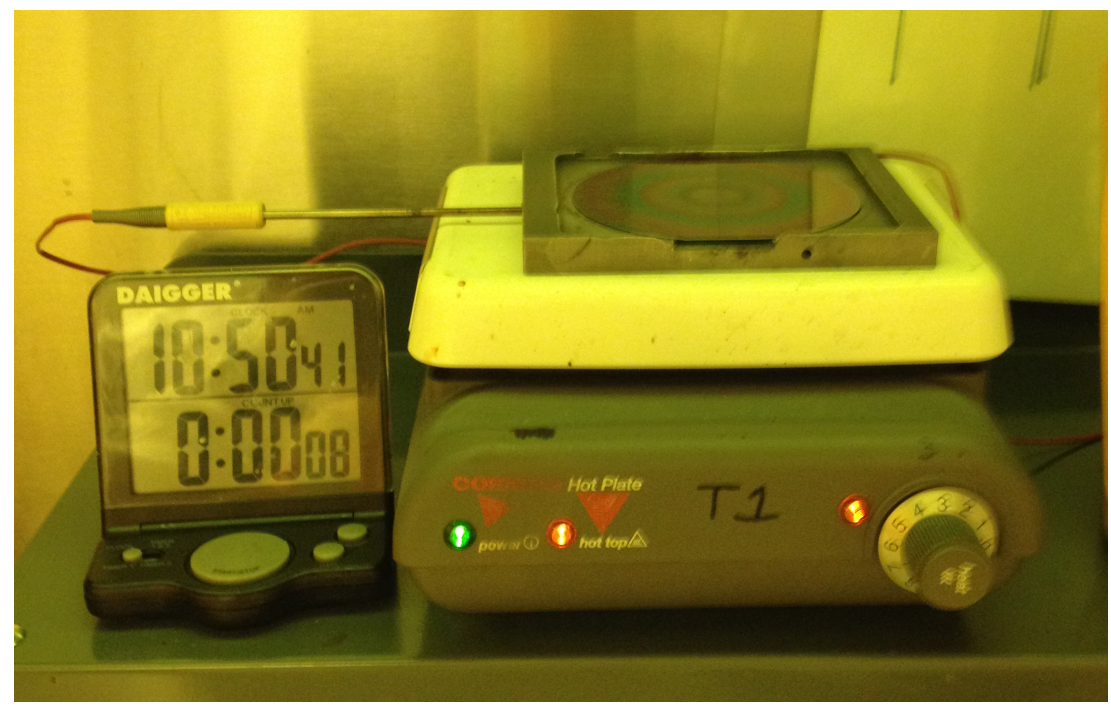

Figure 44: Hot plate used for soft and hard baking of wafers after the application of positive photo resist.

A lithography mask was found in the clean room with 4,5 , and $7 \mathrm{~mm}$ diaphragms (Figure 45). This mask also had break lines between the diaphragms to facilitate removal of individual diaphragms from the wafer. The break lines are visible in Figure 46 as lines of approximately 30um. When placed in the dual doped TMAH solution, the lines 
will etch down, leaving crystallographically oriented lines on which the wafer should preferentially break.

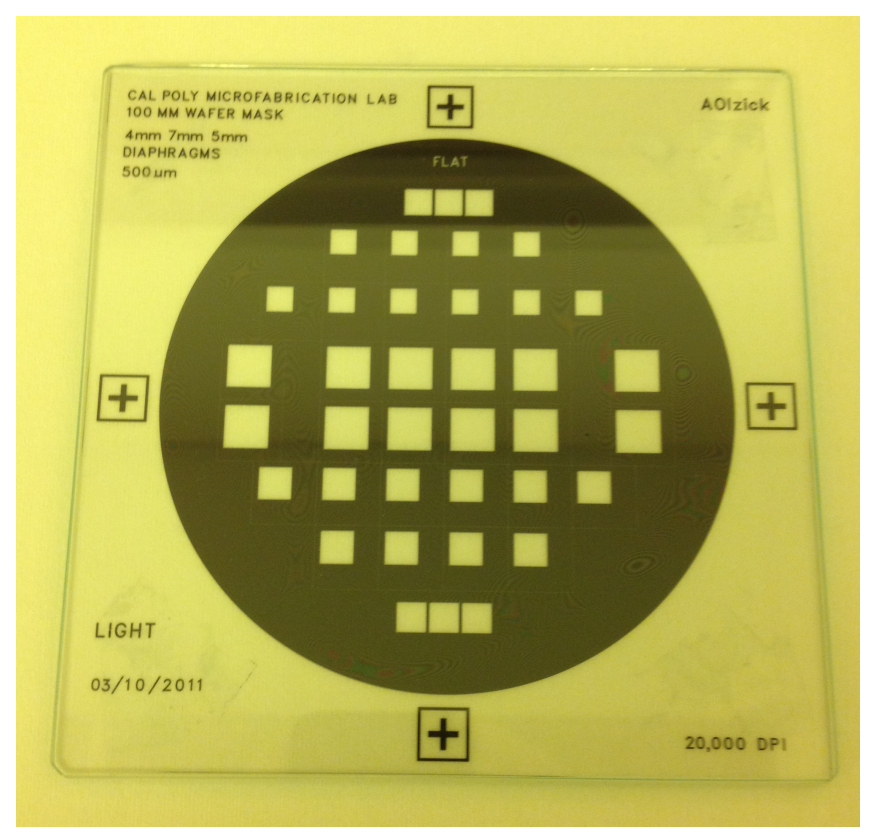

Figure 45: Lithography mask used to create 4, 5, and $7 \mathrm{~mm}$ diaphragms

The "dummy wafer" from each run of the lithography process was used to determine the light integral for each SOI process wafer. A Canon PLA-501FA photolithography machine was used to align and expose the wafers. Light integrals from 1.8 to 3.3 were testing by uncovering only a small portion of the mask for each exposure. For each processing run, a light integral of 2.8 (corresponding to an exposure dose of $50 \mathrm{~mJ} / \mathrm{cm}^{2}$ ) was chosen as the optimum exposure based on optical inspection of the "dummy wafer". Next the SOI process wafer was aligned and exposed using the light integral determined from the "dummy wafer". After the SOI process wafer was exposed, it was developed with Microposit MF CD-26 developer at room temperature for approximately 2 minutes to remove the soluble regions created during the exposure. After an optical inspection to ensure proper pattern transfer, the SOI process wafers 
were placed on a hot plate at $150^{\circ} \mathrm{C}$ to hard bake the wafer, removing latent solvents. The wafer was subsequently optically inspected before the oxide etch (Figure 46).

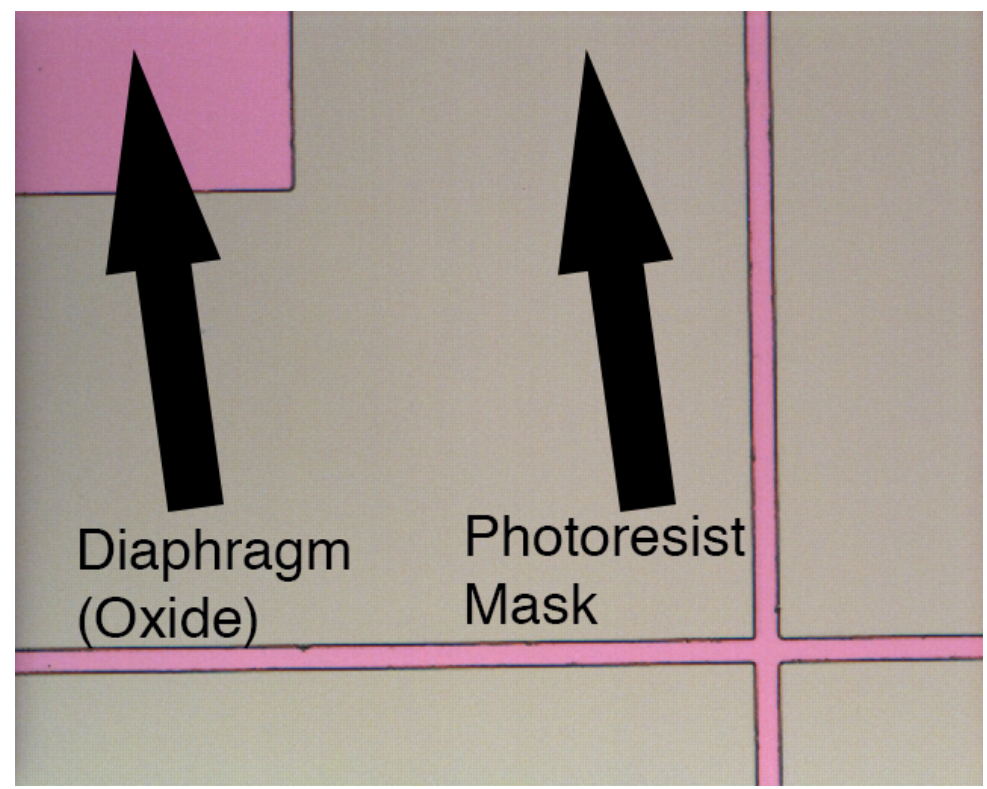

Figure 46: Optical microscopy showing the diaphragm part of the lithography mask as well as the photoresist protecting the oxide around each diaphragm.

\subsection{OXIDE ETCH}

The oxide etch allowed the previously grown thermal oxide to be selectively removed, exposing parts of the silicon handle layer to the dual doped TMAH etch (Figure 47). A single sided etch apparatus was used so as not to damage the thermal oxide covering the device layer of the SOI process wafer (which was not protected by photo resist). 


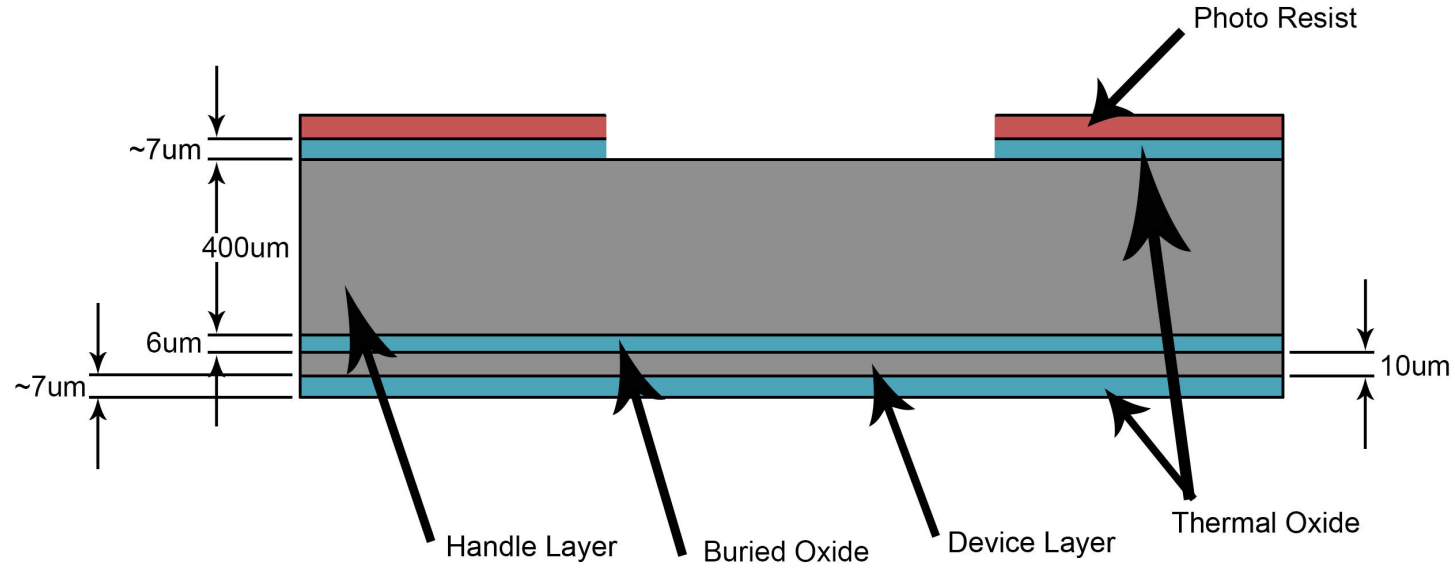

Figure 47: Single sided BOE etch of SOI process wafer to selectively remove the thermally grown oxide on the device side of the SOI wafer.

\subsubsection{BOE ETCH RATE DETERMINATION}

The BOE etch rate was tested by placing a "dummy wafer" which was oxidized with the SOI process wafers to an average oxide thickness of $7041 \AA$. The wafer was placed in a vertical wafer holder, then placed in a BOE bath (Figure 48). The wafer was lowered into the bath $1 \mathrm{~cm}$ every 2 minutes, creating different oxide thicknesses depending on the time in the BOE bath. The wafer was lowered 5 times for maximum etch time of 10 minutes. The Filmetrics F-20 film thickness measurement system was the used to measure the thickness of the oxide at each etch time (Table 13). 


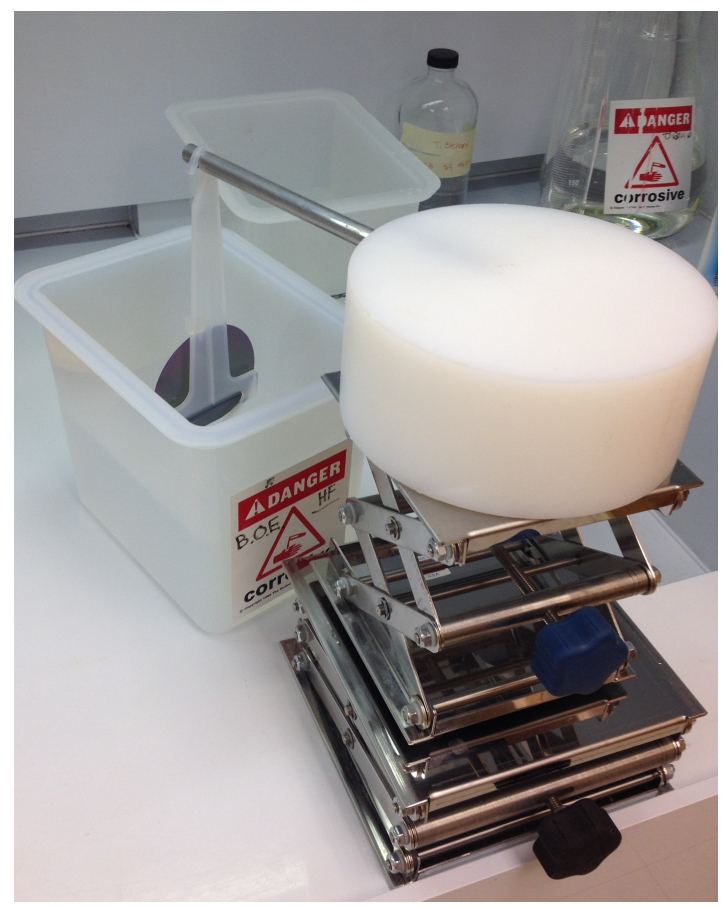

Figure 48: Testing of BOE etch rate.

Table 13: BOE etch rate characterization.

\begin{tabular}{|c|c|c|c|}
\hline Step & Oxide Thickness & $\begin{array}{c}\text { Total BOE Etch } \\
\text { Time (min) }\end{array}$ & $\begin{array}{c}\text { Average Oxide Etch } \\
\text { Rate (A/min) }\end{array}$ \\
\hline 0 (initial thickness) & $7041 \AA$ & 0 & 0 \\
\hline 1 & $4100 \AA$ & 2 & $1470 \AA$ \\
\hline 2 & $1996 \AA$ & 4 & $1052 \AA$ \\
\hline 3 & 0 & 6 & $998 \AA$ \\
\hline 4 & 0 & 8 & -- \\
\hline 5 & 0 & 10 & -- \\
\hline
\end{tabular}

The Observed BOE etch rate varied with time, however, it was higher than the specified etch rate of $800 \AA$. This may be due to the temperature in the lab, or residual BOE remaining on each layer of the test wafer. Because of the high chemical resistance of the positive photo resist oxide etch mask, an etch time of 12 minutes was chosen to ensure complete removal of the oxide layer. 


\subsubsection{BOE OXIDE ETCH PROCEDURE}

With the characterized etch rate, the SOI process wafers were loaded into the single sided etching apparatus (Figure 49) with the photo resist covered handle side of the SOI wafer facing up. The bolts of the apparatus were tightened (Figure 50) such that the force applied to the O-ring on the top side of the wafer was evenly distributed, ensuring no leakage of BOE onto the backside of the wafer. A pipet was used to apply a small amount of BOE $\sim 5 \mathrm{~mL}$ to the surface of the wafer. The wafer was etched (Figure 51) for 12 minutes, then quenched in DI water to stop the etching process.

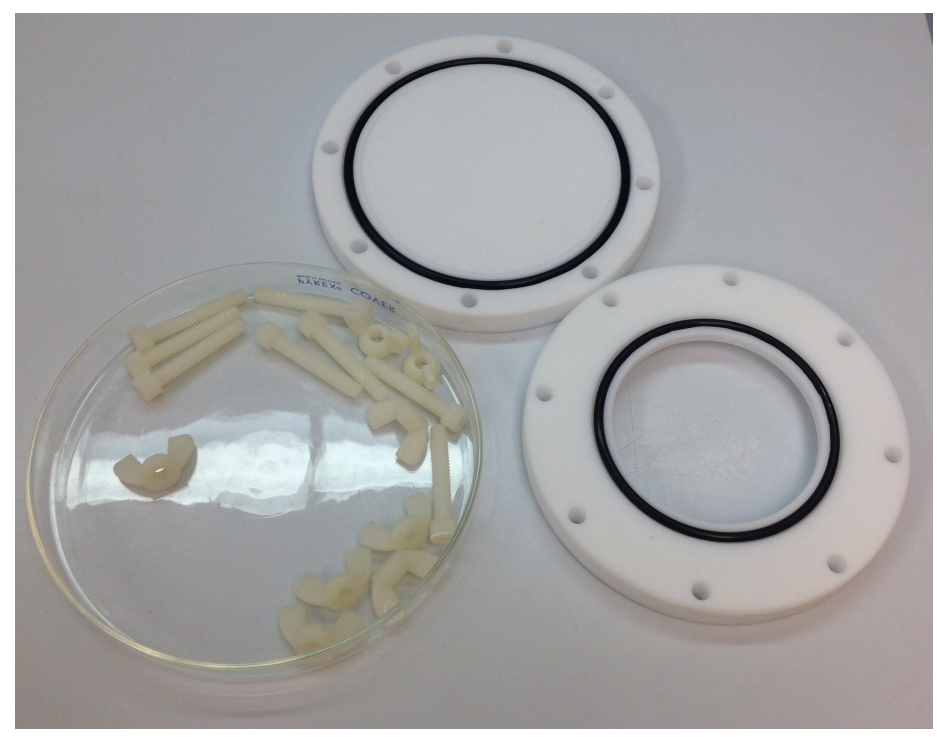

Figure 49: Single Sided etching apparatus used for BOE oxide etching. 


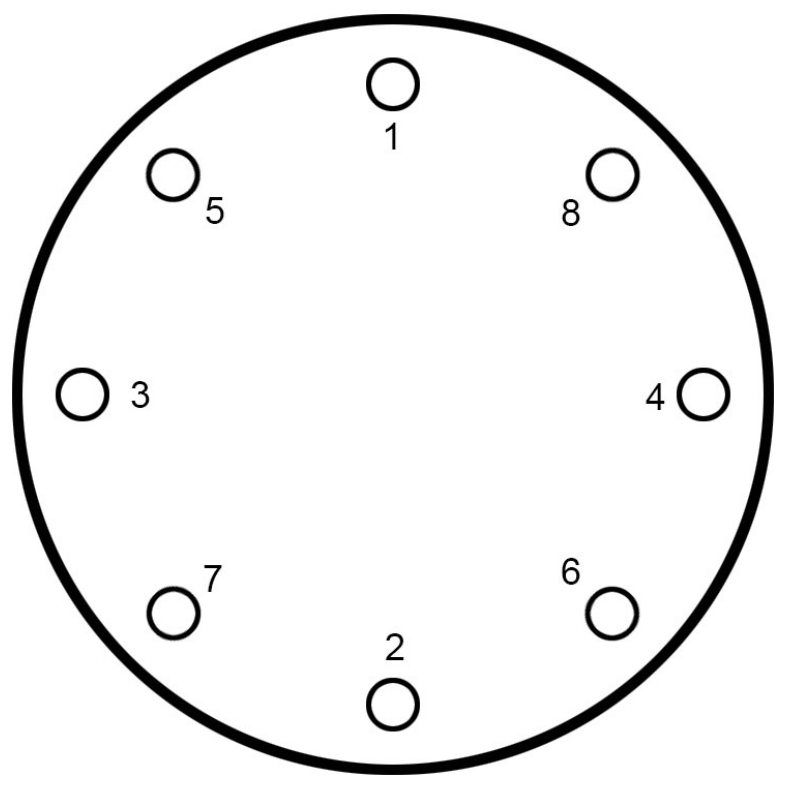

Figure 50: Torque pattern to ensure no BOE leaked past the O-ring of the single sided etching apparatus.

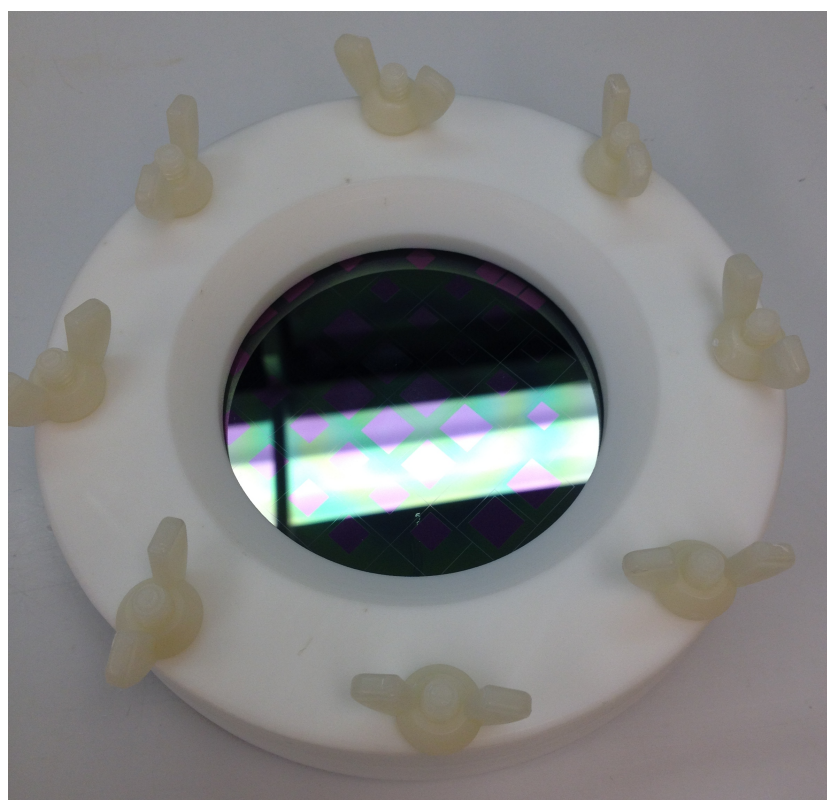

Figure 51: BOE etch of oxide layer on handle side of SOI process wafer. Diaphragms are visible as light purple squares, and positive photo resist is visible as the surrounding green areas.

After the SOI process wafers were removed from the single sided etching apparatus, the wafers were placed in Microposit remover 1165 at room temperature for 5 
minutes to remove the positive photo resist (Figure 52 and Figure 53). Subsequently the wafers were cleaned in a 9:1 Piranha solution, then spin rinsed and dried (Figure 54). The SOI process wafers were then placed in the Filmetrics F-20 thin film thickness measurement system to ensure that the entire oxide layer had been removed from the diaphragm windows in the positive photo resist mask. The results indicated the entire oxide layer had been selectively removed from the wafer (Figure 55).
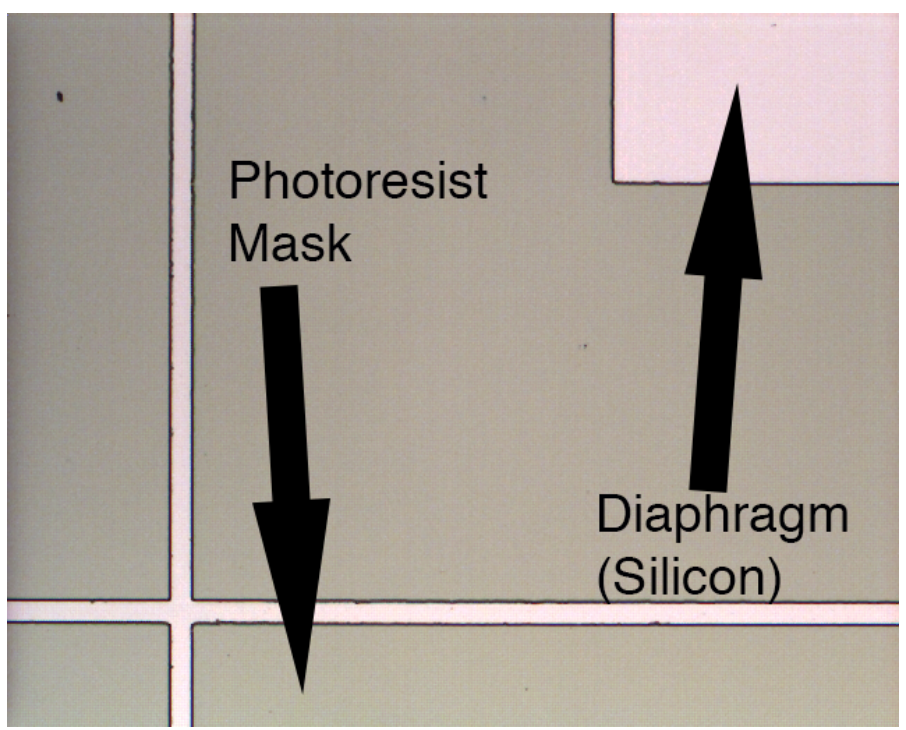

Figure 52: Optical microscopy showing a close up of a diaphragm and break lines. Photoresist is still masking the wafer. 

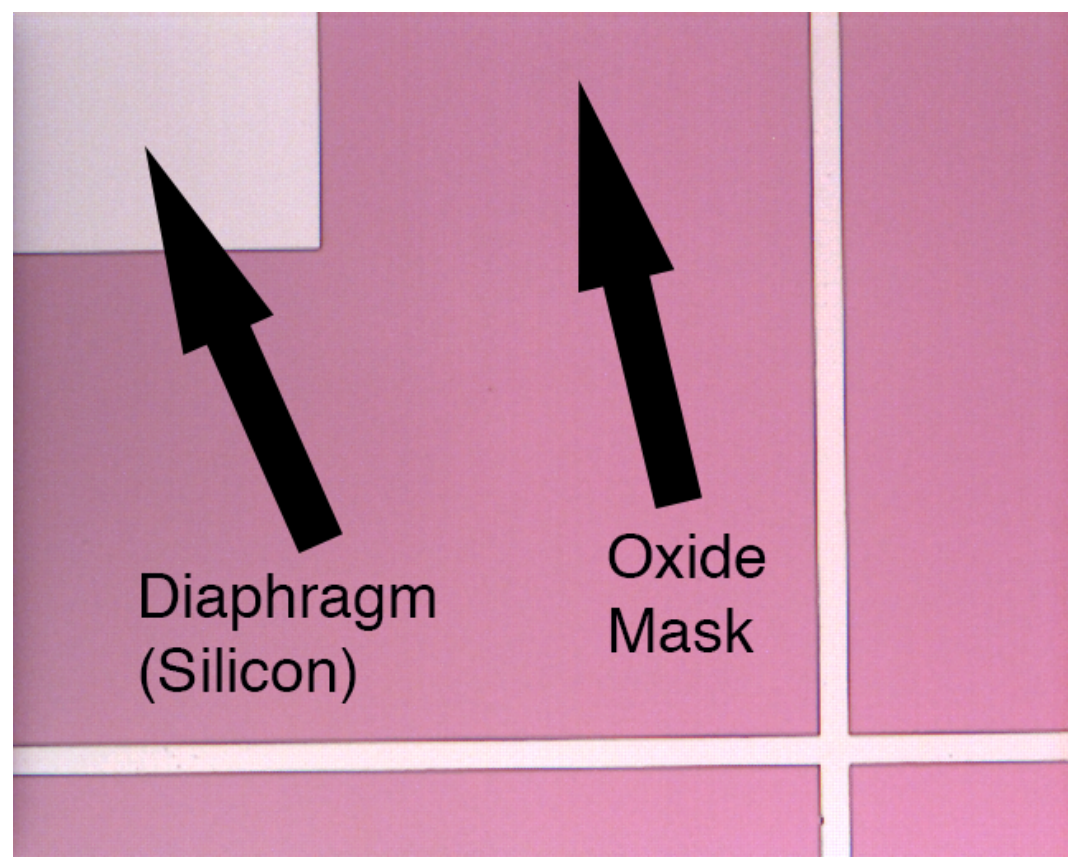

Figure 53: Optical microscopy showing the diaphragm and break lines of the wafer after the positive photoresist had been removed from the wafer.

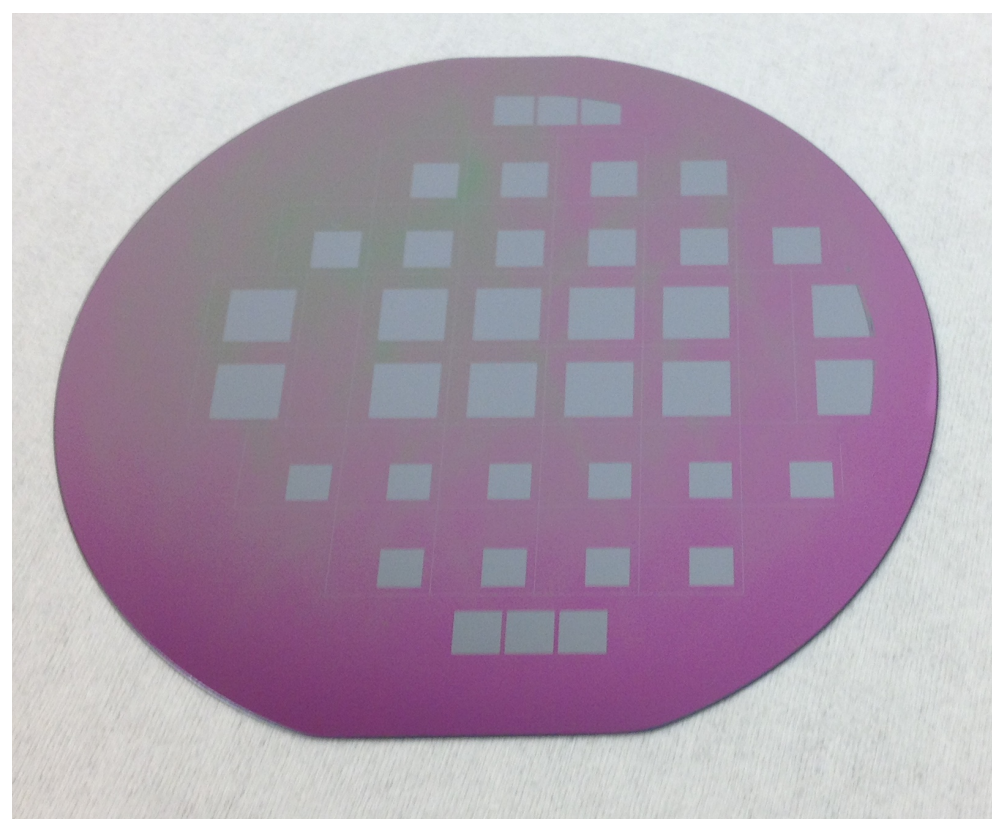

Figure 54: SOI Process wafer after single sided BOE etch. The handle side of the SOI process wafer is shown. Grey regions represent bare silicon, while purple regions represent oxide. The second SOI process wafer (shown) had slight misalignment of the lithography mask making 3 of the diaphragms unusable (visible on the top and right side of the wafer). 


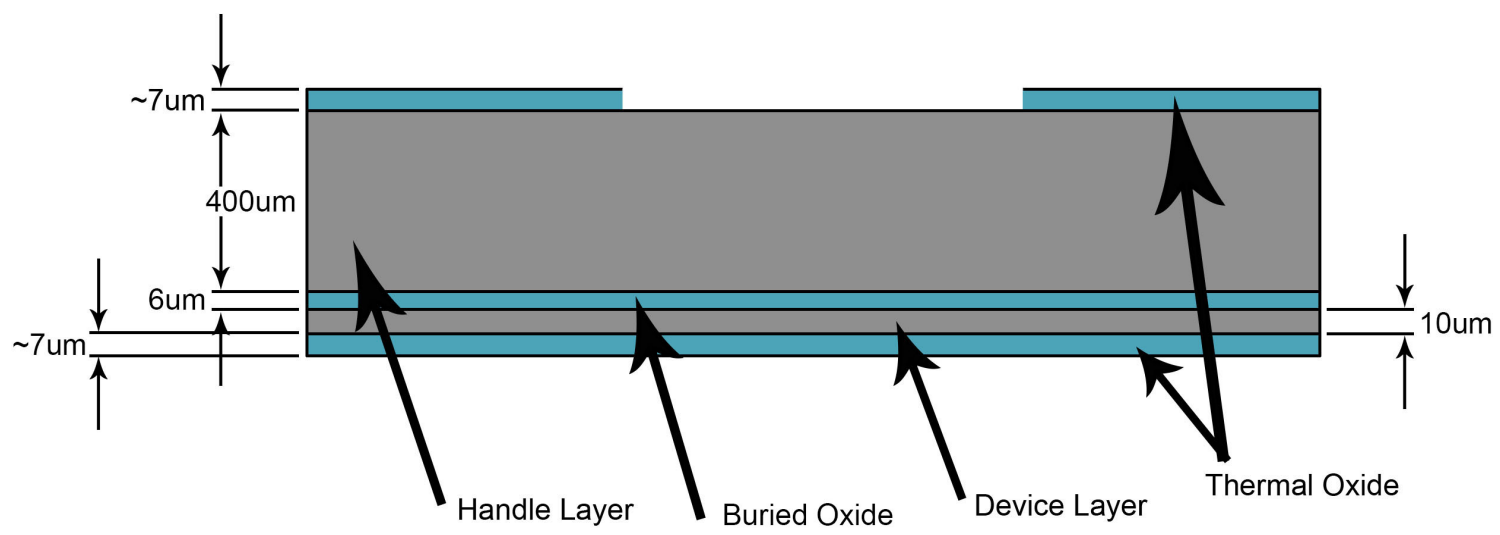

Figure 55: Removal of positive photo resist from handle side of the SOI process wafer.

\subsection{DUAL DOPED TMAH WET ETCH}

The purpose of the dual doped tetra-methyl ammonium hydroxide (TMAH) wet etch was to remove the exposed silicon from the handle layer of the SOI process wafers (Figure 56). TMAH is an anisotropic etch for micromachining silicon, which has high selectivity based on the crystallographic planes of the substrate. At $80^{\circ} \mathrm{C}$ a $5 \% \mathrm{TMAH}$ solution in water will exhibit etch ratios of 20:35:1 for the (100):(110):(111) crystal planes respectively. ${ }^{21}$ The major issue with TMAH is the formation of crystal hillocks on the (100) plane. This formation reduces the etch rate as well as increases the surface roughness. To reduce the formation of hillocks, and reduce the overall rate of the reaction (so as to make the etch more manageable) two additives were added to the TMAH solution (hence dual doped TMAH). Silicic acid was added to reduce the $\mathrm{pH}$ of the solution, reducing the reactivity of the etch, and making the etch rate more consistent for the duration of the immersion. Ammonium persulfate (AP) was added to improve the (100) etch smoothness, as well as remove etch products from the surface of the wafer. ${ }^{22}$ 
$5 \%$ TMAH was used due to its availability in the lab, with $38 \mathrm{~g} / /$ silicic acid, and $7 \mathrm{~g} / \mathrm{l}$ AP added. $^{15}$

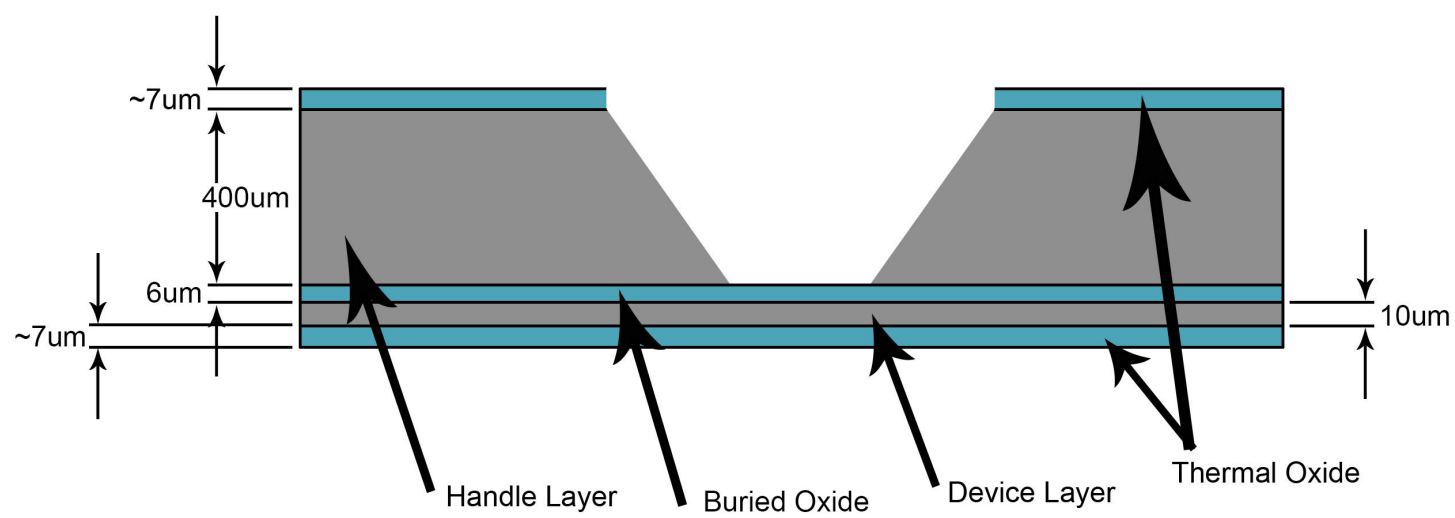

Figure 56: A dual doped TMAH solution was used to selectively remove silicon from the handle layer of the SOI process wafers.

\subsubsection{PROCEDURE}

The Etching solution was comprised of $2.5 \mathrm{~L}$ of $5 \%$ TMAH with $95 \mathrm{~g}$ silicic acid and $17.5 \mathrm{~g} \mathrm{AP}$. The TMAH was added to the beaker at room temperature along with the silicic acid. The solution was then heated with the heating blanket and hot plate to $75^{\circ} \mathrm{C}$ with the stir bar set at approximately 300RPM (hot plate setting of 8). Once at temperature, the AP was added to the solution. The remaining silicic acid and AP powder was completely dissolved after approximately 30 minutes at $75^{\circ} \mathrm{C}$. Due to the length of the etch, the process wafers were etched in two steps (to ensure the wafer did not remain in the solution for too long after reaching the buried oxide etch stop). Once the solution became clear, each process wafer was loaded into the etch chamber (Figure 57). 


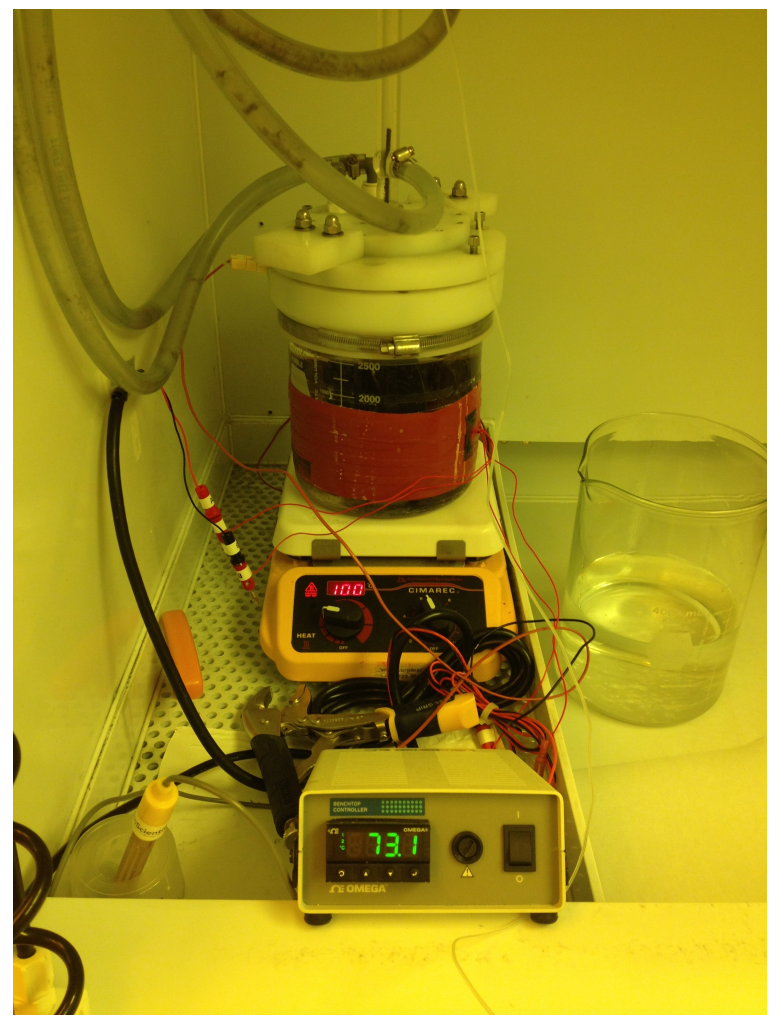

Figure 57: Dual doped TMAH etching condenser chamber.

The SOI process wafers were suspended in the solution using a Teflon single wafer holder. To ensure the removal of etchant products, as well as ensure constant contact of etchant with the surface of the wafer, the wafers were suspended vertically. The wafers were visually inspected through the beaker to determine the end of the etch process (Figure 58). 


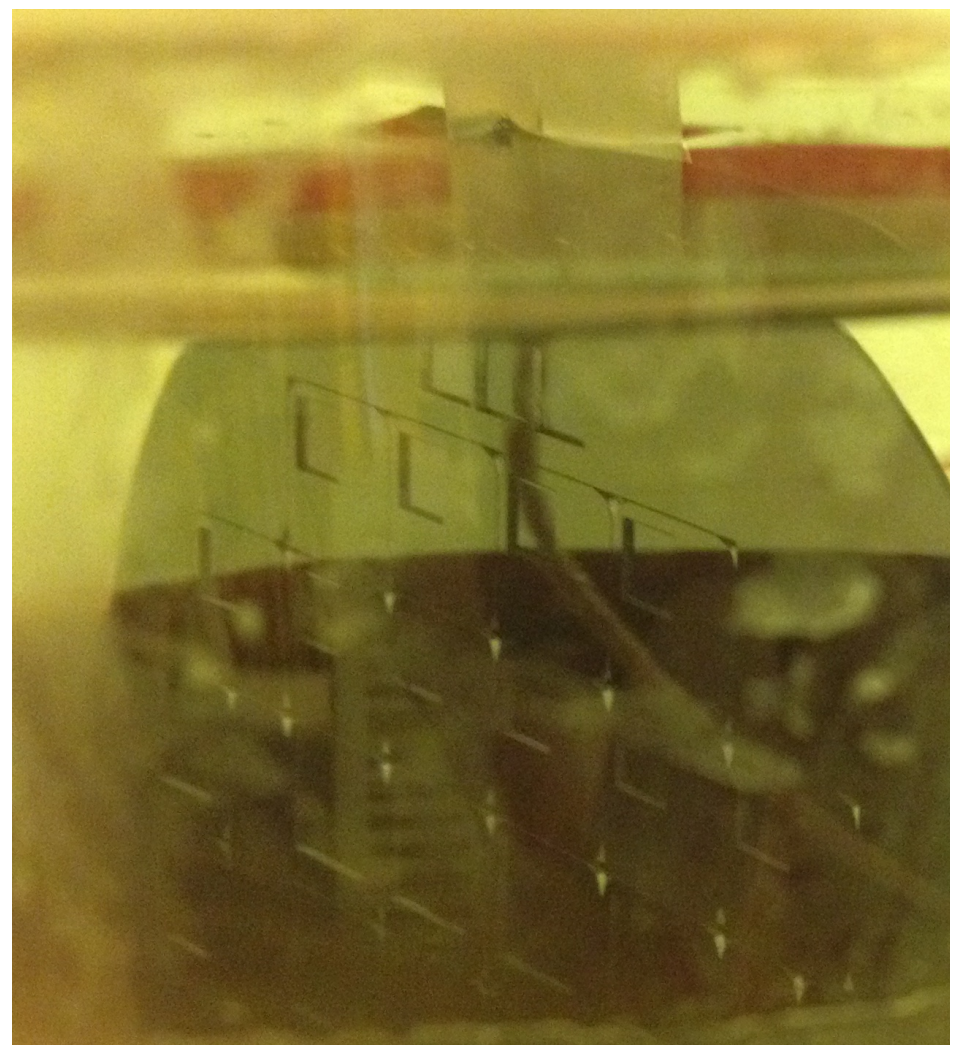

Figure 58: Visual inspection of vertically mounted SOI process wafer during dual doped TMAH etch. The buried oxide layer was visible once the handle side silicon had been fully removed.

Once the buried oxide layer had been reached, the wafers were gently removed from the etching chamber, and rinsed with DI water. Final etch times for process wafer 1 and 2 were 10 hours and 9 hours respectively (Table 14).

Table 14: Dual doped TMAH etch times for SOI process wafers.

\begin{tabular}{|l|l|l|}
\hline Time & SOI Process Wafer \#1 & SOI Process Wafer \#2 \\
\hline Etch\#1 & $\sim 8$ hours & $\sim 7$ hours \\
\hline Etch\#2 & $\sim 2$ hours & $\sim 2$ hours \\
\hline
\end{tabular}

After quenching the diaphragms in a beaker of DI water, warpage was visibly evident on the diaphragms due to stresses in the buried and thermal oxides (Figure 59). 


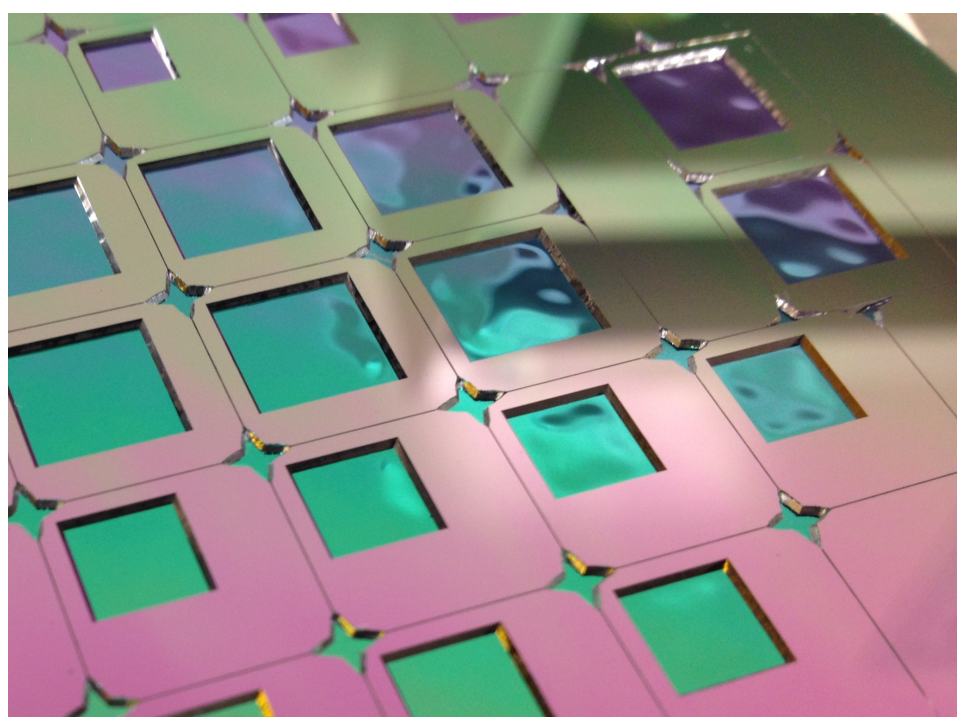

Figure 59: Diaphragms after dual doped TMAH etch, warpage is visible due to the difference in film thickness of the buried and thermal oxide.

The warpage was first measured using a profilometer, however due to the thickness of the diaphragms, the results were skewed by the profilometer tip deflecting the diaphragms to a greater extent than the warpage itself. The extent of the warpage was measured using a Bruker NPFLEX Interferometer (located in the Environmental Engineering Department at Cal Poly) for a passive approach to characterizing the warpage.

\subsubsection{INTERFEROMETER ANALYSIS OF RESULTS}

One $7 \mathrm{~mm}$ diaphragm from process wafer 2 was scanned using a Bruker interferrometer. Due to the size of the diaphragm, a 9 by 9 grid of scans was used to cover a $7.5 \mathrm{~mm}^{2}$ area. A 3 dimensional view was created showing the warpage of the diaphragm (Figure 60), and a cross section in the middle of the diaphragm was used to find the maximum warpage of 67um (Figure 61). 


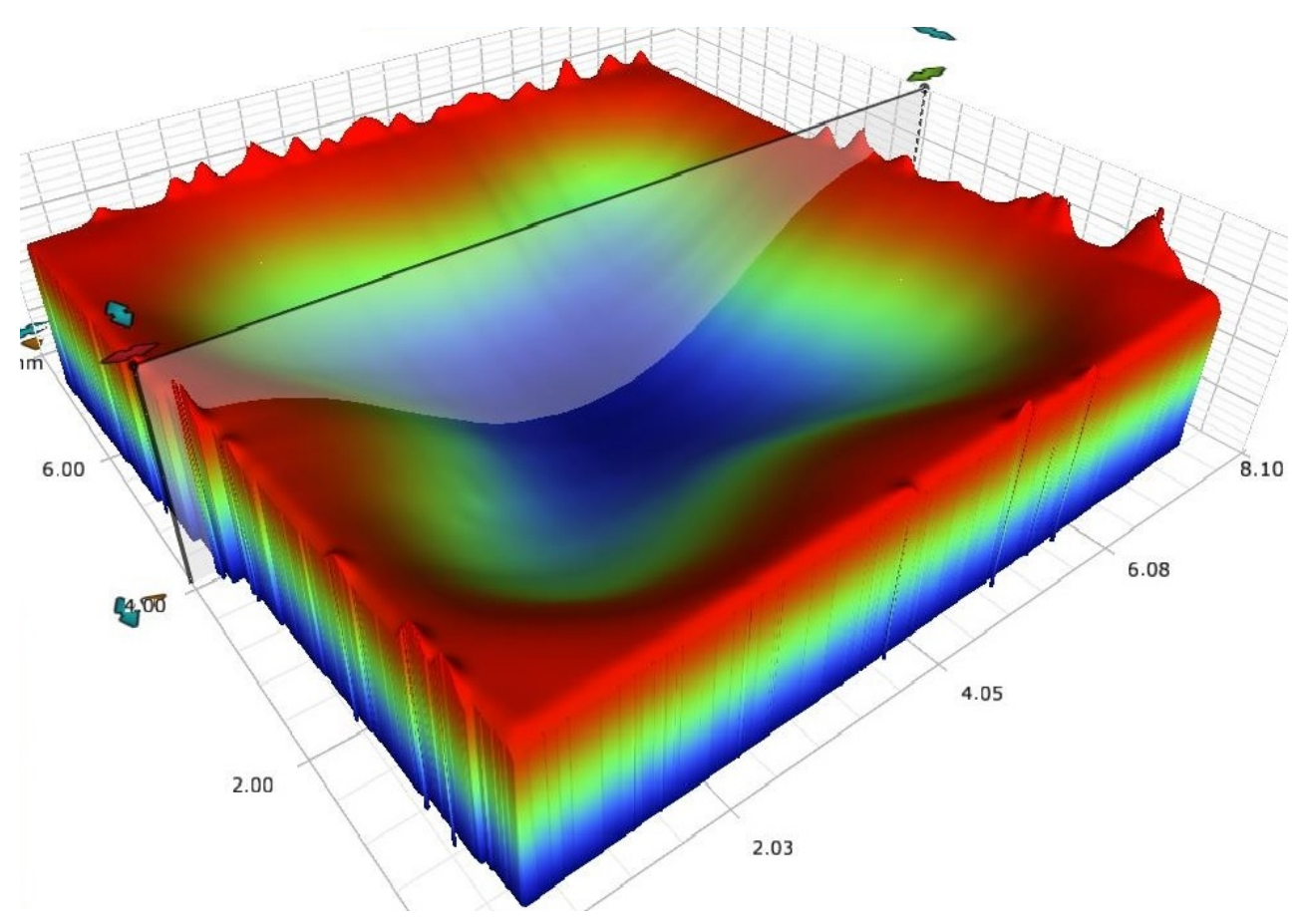

Figure 60: 3 dimensional plot generated from Bruker interferometer showing the warpage from the buried and thermal oxide layers.

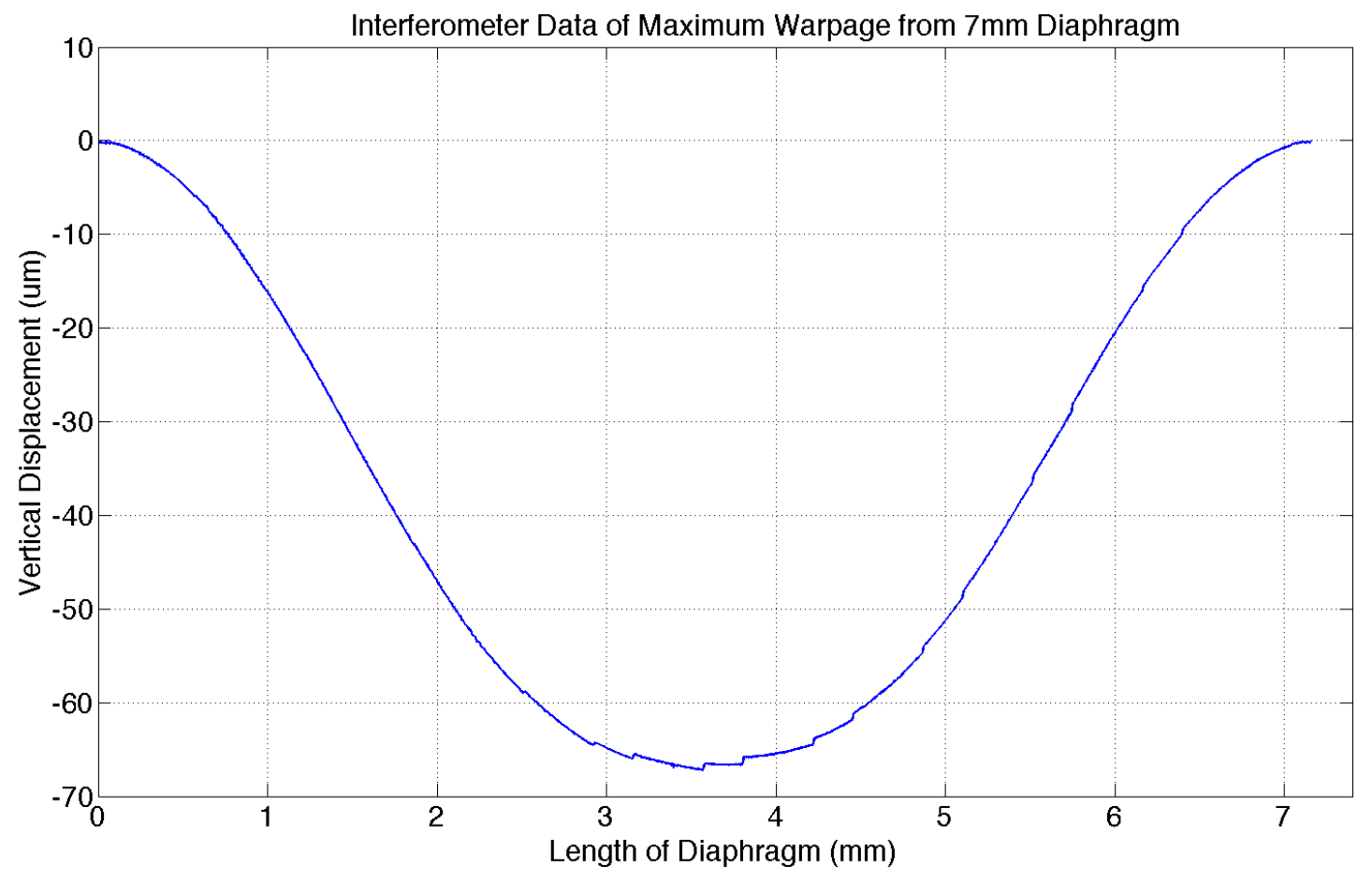

Figure 61: Interferometer data from y direction of $7 \mathrm{~mm}$ diaphragm before the removal of the burried and themal oxide layers. The maximum warpage of this diaphragm was 67 um. 
Using the center warpage from the interferometer results (67 um), and outputting the maximum stress at the center according to Eq. 11, the maximum differential stress induced by the films on the silicon was found to be $147 \mathrm{MPa}$, using a point force as opposed to a pressure $(51.8 \mathrm{MPa})^{16}$. Both of these values were found to be well below the fracture strength of silicon (4.82 GPa).

\subsection{OXIDE REMOVAL}

The wafers were etched in BOE to remove the thermally grown oxide as well as buried oxide (Figure 62). This process relieved the stress created by the film thickness differences in the buried and thermal oxide of the device side of the wafer, mitigating the visible warpage.

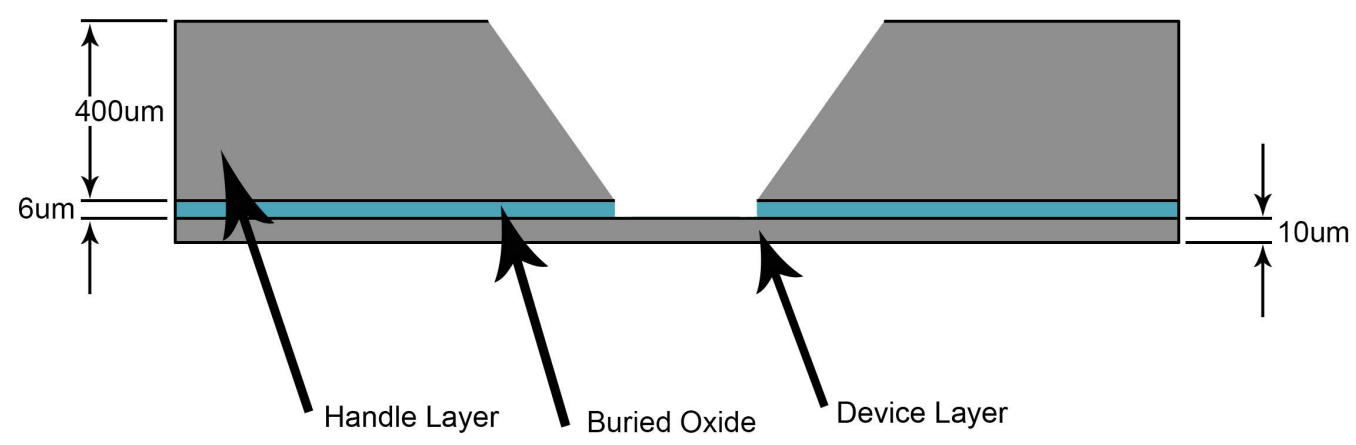

Figure 62: Removal of thermally grown oxide on the handle side and device side of the wafer as well as the buried oxide beneath the etched silicon completed the wafer processing.

The same $7 \mathrm{~mm}$ diaphragm from the second process wafer was again placed in the Bruker interferometer to measure the flatness of the diaphragm after the removal of the buried and thermal oxides. The same 3 dimensional view was created to show the relative warpage as compared to pre-oxide removal (Figure 63). The data suggests that 
the diaphragms only had about $250 \mathrm{~nm}$ of warpage as compared to 67 um prior to the removal of the oxide layers (Figure 64). The interferometer data exhibits artifacts from the stitching process, visible as the local maximums in Figure 64.

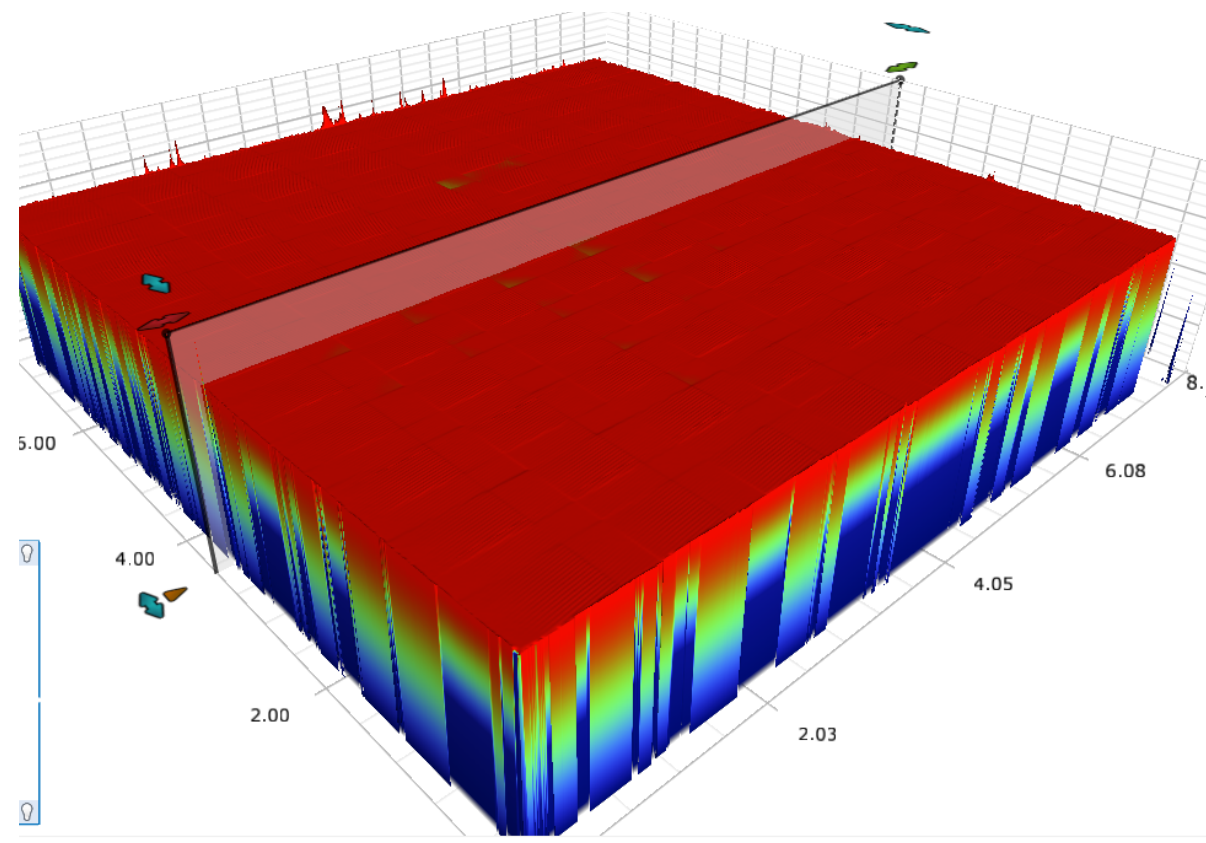

Figure 63: 3 dimensional plot generated from Bruker interferometer showing the no warpage after the removal of the buried and thermal oxide layers. 


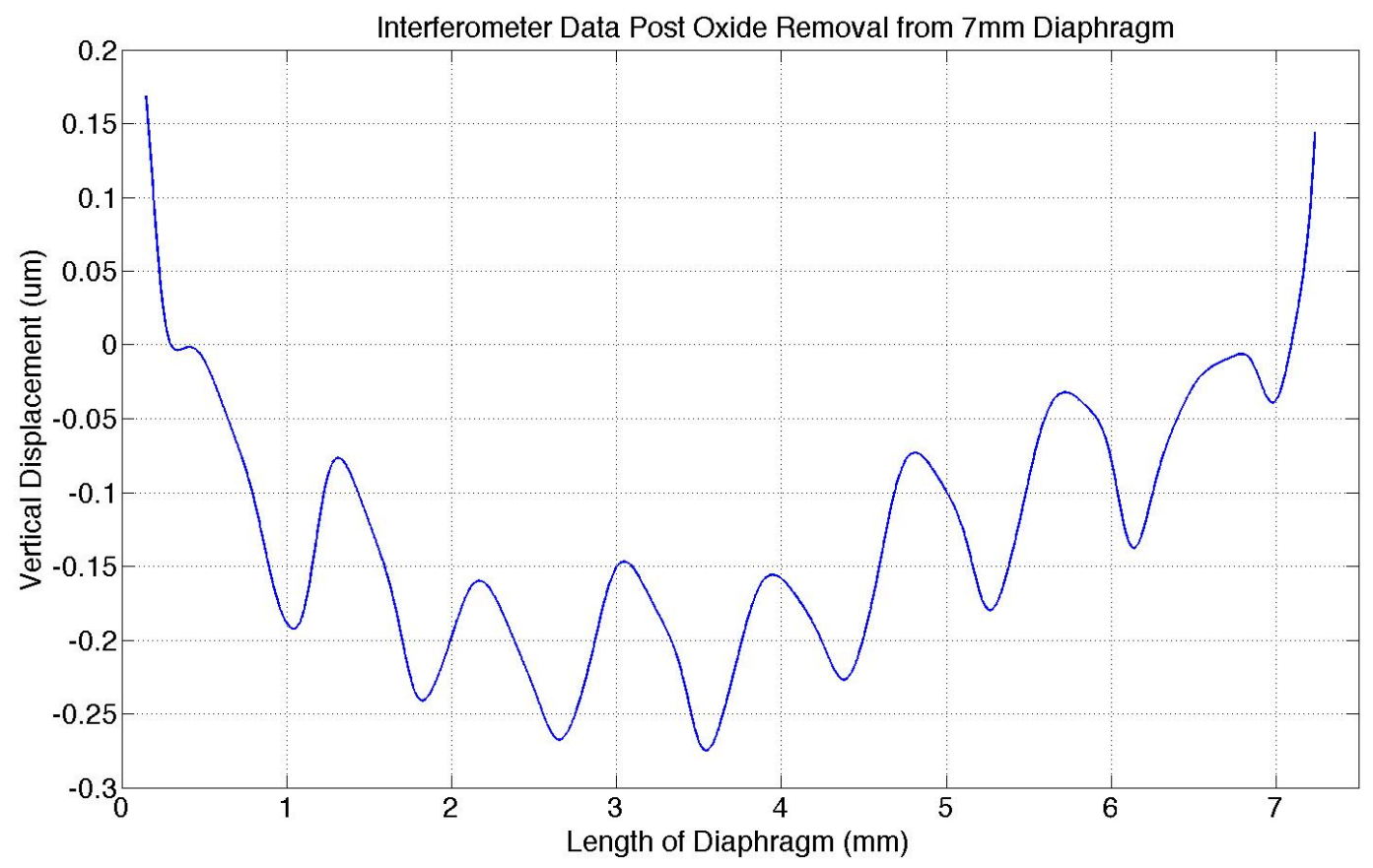

Figure 64: Interferometer cross sectional scan data after buried and thermal oxides had been removed from the wafer. Curves in the data were caused by the stitching of many scans to create the overall size. The warpage is approximately $250 \mathrm{~nm}$, which is partially accountable through the weight of the diaphragm itself.

\subsection{DIAPHRAGM CHARACTERIZATION}

Each diaphragm was visually analyzed using an optical microscope to search for any pin holes created by defects in the SOI buried oxide layer. Due to the anisotropic nature of the dual doped TMAH etch, the pin holes are square in shape and have a side length of approximately 30um (Figure 65). 

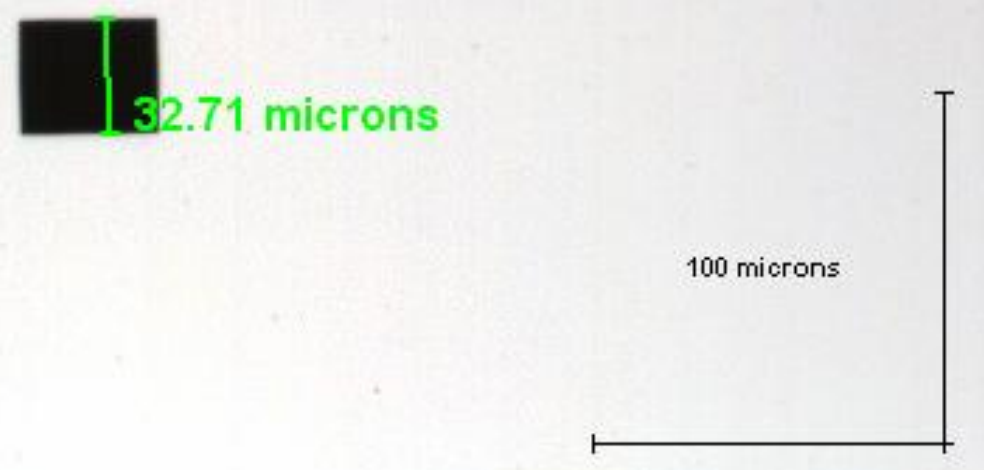

Figure 65: Example of a pinhole found on many of the diaphragms.

A naming scheme was created to designate each diaphragm on the SOI process wafers (Figure 66). The locations of the pin holes within the diaphragm were divided into three sections of the diaphragm (Figure 67). A list of all defects was created (Table 15). The number preceding the $\mathrm{X}$ represents the number of defects in each designated region. 


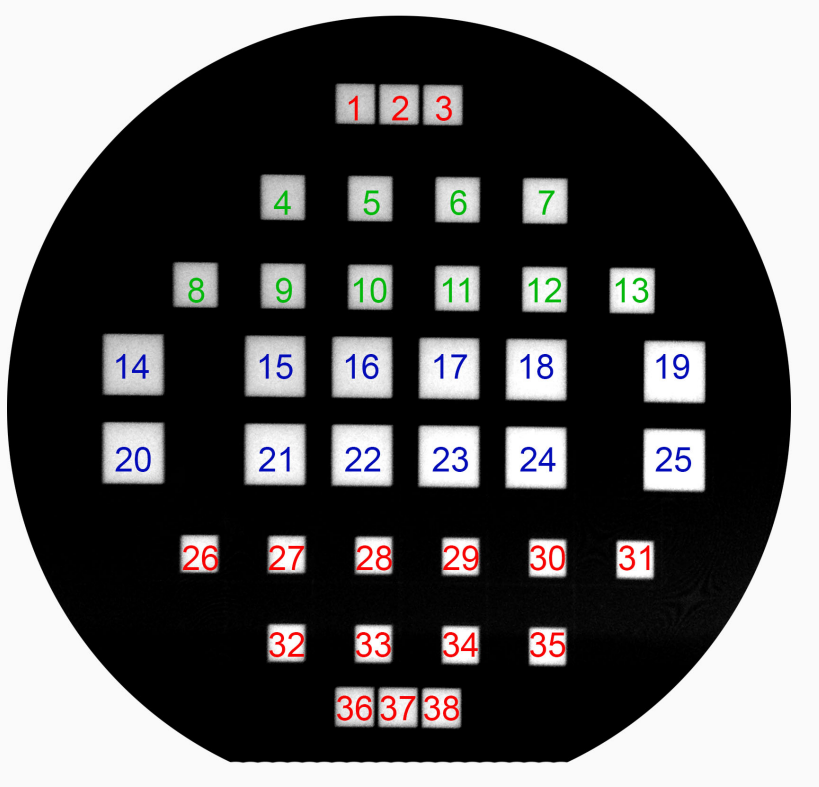

Figure 66: Diaphragm locations, $4 \mathrm{~mm}$ (red), $5 \mathrm{~mm}$ (green), and $7 \mathrm{~mm}$ (blue) diaphragms.

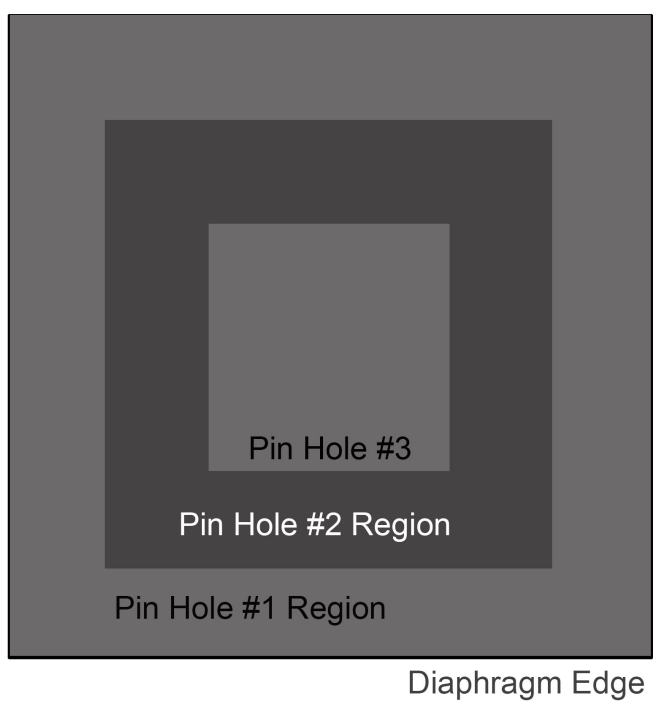

Figure 67: Pin hole location nomenclature. 
Table 15: Diaphragm Defects

\begin{tabular}{|c|c|c|}
\hline Location & SOI Process Wafer \#1 & SOI Process Wafer \#2 \\
\hline 1 & $1 \mathrm{XPH} 2$ & CLEAN \\
\hline 2 & $1 \mathrm{XPH} 1$ & CLEAN \\
\hline 3 & CLEAN & Incomplete* \\
\hline 4 & $2 \mathrm{XPH} 2$ & CLEAN \\
\hline 5 & 1XPH1,1X PH2 & CLEAN \\
\hline 6 & CLEAN & CLEAN \\
\hline 7 & CLEAN & $1 \mathrm{XPH} 1$ \\
\hline 8 & $1 \mathrm{XPH} 1$ & CLEAN \\
\hline 9 & $1 \mathrm{XPH} 1$ & $1 \mathrm{XPH} 1$ \\
\hline 10 & 4XPH1, 3XPH2 & $1 \mathrm{XPH} 1$ \\
\hline 11 & CLEAN & CLEAN \\
\hline 12 & CLEAN & 1XPH1 \\
\hline 13 & CLEAN & 1XPH2 \\
\hline 14 & $2 \mathrm{XPH} 3$ & 1XPH1, 1XPH3 \\
\hline 15 & CLEAN & $1 \mathrm{XPH} 2$ \\
\hline 16 & CLEAN & $1 \mathrm{XPH} 1$ \\
\hline 17 & $2 \mathrm{XPH} 1$ & CLEAN \\
\hline 18 & $1 \mathrm{XPH} 2,2 \mathrm{XPH} 3$ & $3 \mathrm{XPH} 1$ \\
\hline 19 & CLEAN & Incomplete ${ }^{*}$ \\
\hline 20 & $1 \mathrm{XPH} 1$ & CLEAN \\
\hline 21 & $2 \mathrm{XPH} 1$ & $1 \mathrm{XPH} 2$ \\
\hline 22 & 1XPH1 & $1 \mathrm{XPH} 1$ \\
\hline 23 & CLEAN & CLEAN \\
\hline 24 & CLEAN & CLEAN \\
\hline 25 & $2 \mathrm{XPH} 1,1 \mathrm{XPH} 3$ & Incomplete* \\
\hline 26 & CLEAN & CLEAN \\
\hline 27 & CLEAN & $1 \mathrm{XPH} 1$ \\
\hline 28 & $1 \mathrm{XPH} 1$ & 1XPH2 \\
\hline 29 & CLEAN & CLEAN \\
\hline 30 & CLEAN & CLEAN \\
\hline 31 & CLEAN & $1 \mathrm{XPH} 1$ \\
\hline 32 & SEM TESTING & SEM TESTING \\
\hline 33 & CLEAN & 1XPH1, 2XPH2 \\
\hline 34 & CLEAN & CLEAN \\
\hline 35 & CLEAN & CLEAN \\
\hline 36 & CLEAN & CLEAN \\
\hline 37 & CLEAN & CLEAN \\
\hline 38 & CLEAN & $1 \mathrm{XPH} 1$ \\
\hline
\end{tabular}

*Incomplete- Diaphragms not square due to lithography mask misalignment of the second process wafer. 
A bar chart of the data (Figure 68) was created to visualize the number of defects present in each region. Although it appears that the $\mathrm{PH} 1$ region contains more defects than $\mathrm{PH} 2$ and $\mathrm{PH} 3$, the $\mathrm{PH} 1$ region contains the most area, so it should contain the most randomly distributed defects. There does not seem to be a pattern of defects within or between the wafers.

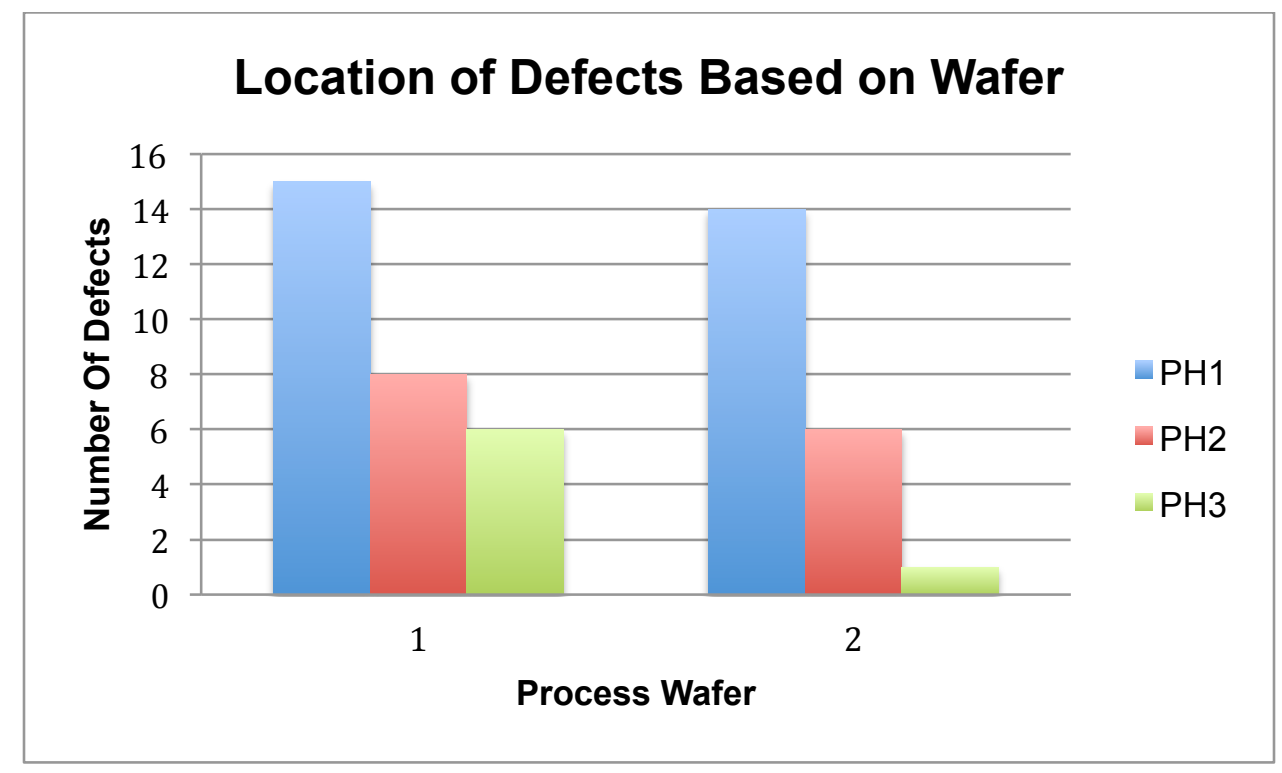

Figure 68: Bar chart of location of defects based on region.

The actual $\mathrm{X}$ and $\mathrm{Y}$ dimensions of the diaphragms were found by placing the diaphragms in the Ambios Profilometer, then scanning the diaphragms in the $\mathrm{X}$ and $\mathrm{Y}$ direction. 3 diaphragms of each size from each of the process wafers were measured. The wafers were visually aligned to be as straight as possible, however the minimum length of 3 samples was chosen to most closely represent the true side lengths of the diaphragms (Table 16). 
Table 16: $X$ and $Y$ lengths of diaphragms from both process wafers.

\begin{tabular}{|l|l|l|l|l|}
\hline $\begin{array}{l}\text { Process } \\
\text { Wafer } \\
\text { Number }\end{array}$ & $\begin{array}{l}\text { Diaphragm } \\
\text { Size }\end{array}$ & $\begin{array}{l}\text { Minimum X Length } \\
\mathbf{( m m})\end{array}$ & $\begin{array}{l}\text { Minimum Y } \\
\text { Length } \mathbf{( m m})\end{array}$ & $\begin{array}{l}\text { Percent Difference } \\
\text { from Nominal } \\
\text { Length }\end{array}$ \\
\hline 1 & 4 & 4.056 & 4.072 & $1.40 \%$ \\
\hline 1 & 5 & 5.003 & 5.040 & $0.06 \%$ \\
\hline 1 & 7 & 7.026 & 7.040 & $0.37 \%$ \\
\hline 2 & 4 & 4.139 & 4.110 & $2.75 \%$ \\
\hline 2 & 5 & 5.110 & 5.100 & $2.00 \%$ \\
\hline 2 & 7 & 7.113 & 7.050 & $0.71 \%$ \\
\hline
\end{tabular}

Cross sectional SEM images of the diaphragms were created by scoring along the middle of the handle layer, then breaking the diaphragms on a glass microscope slide. Several diaphragms were broken to achieve clean enough breaks for SEM imaging. Of most interest was the thickness of the diaphragms. Multiple images were analyzed for the diaphragm thickness (Figure 69) however, the average of the results indicated that the actual thickness was indistinguishable from the nominal thickness of 10um. Future modeling and calculations utilize the nominal thickness.

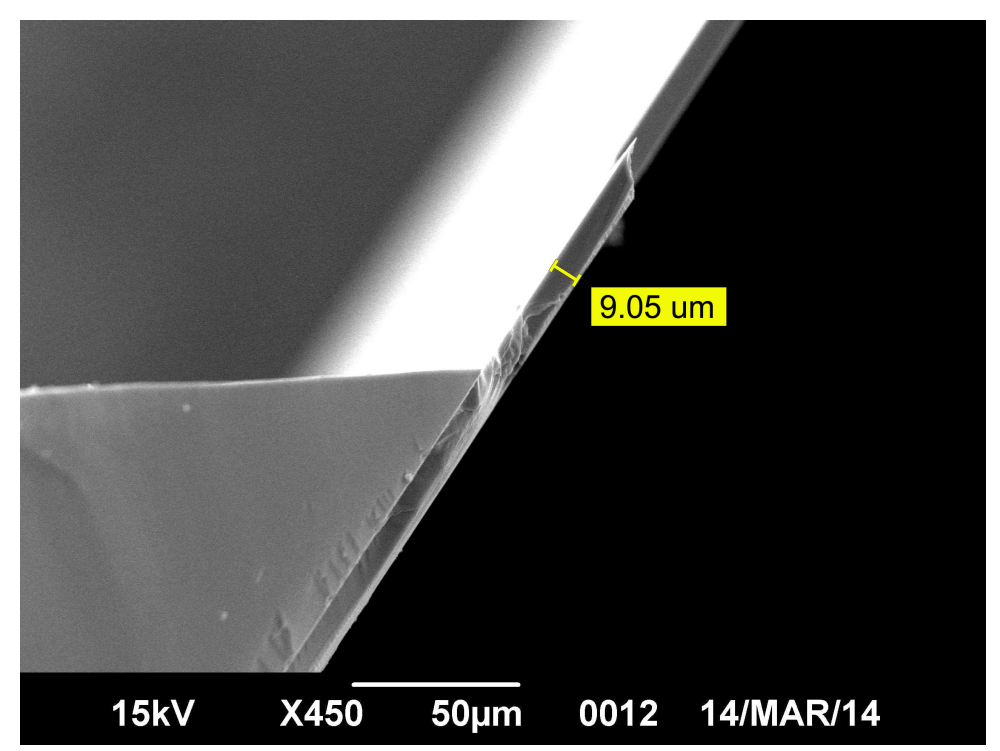

Figure 69: SEM cross-section of process wafer 2, the diaphragm thickness measured was 9.05 um. The image was taken at $15 \mathrm{kV}, 450 \mathrm{X}$ magnification, a working distance of $11 \mathrm{~mm}$, and a spot size of 20.

In addition to diaphragm thickness, the sidewall angle was measured to compare to the theoretical sidewall angle of $54.74^{\circ}{ }^{23}$ 


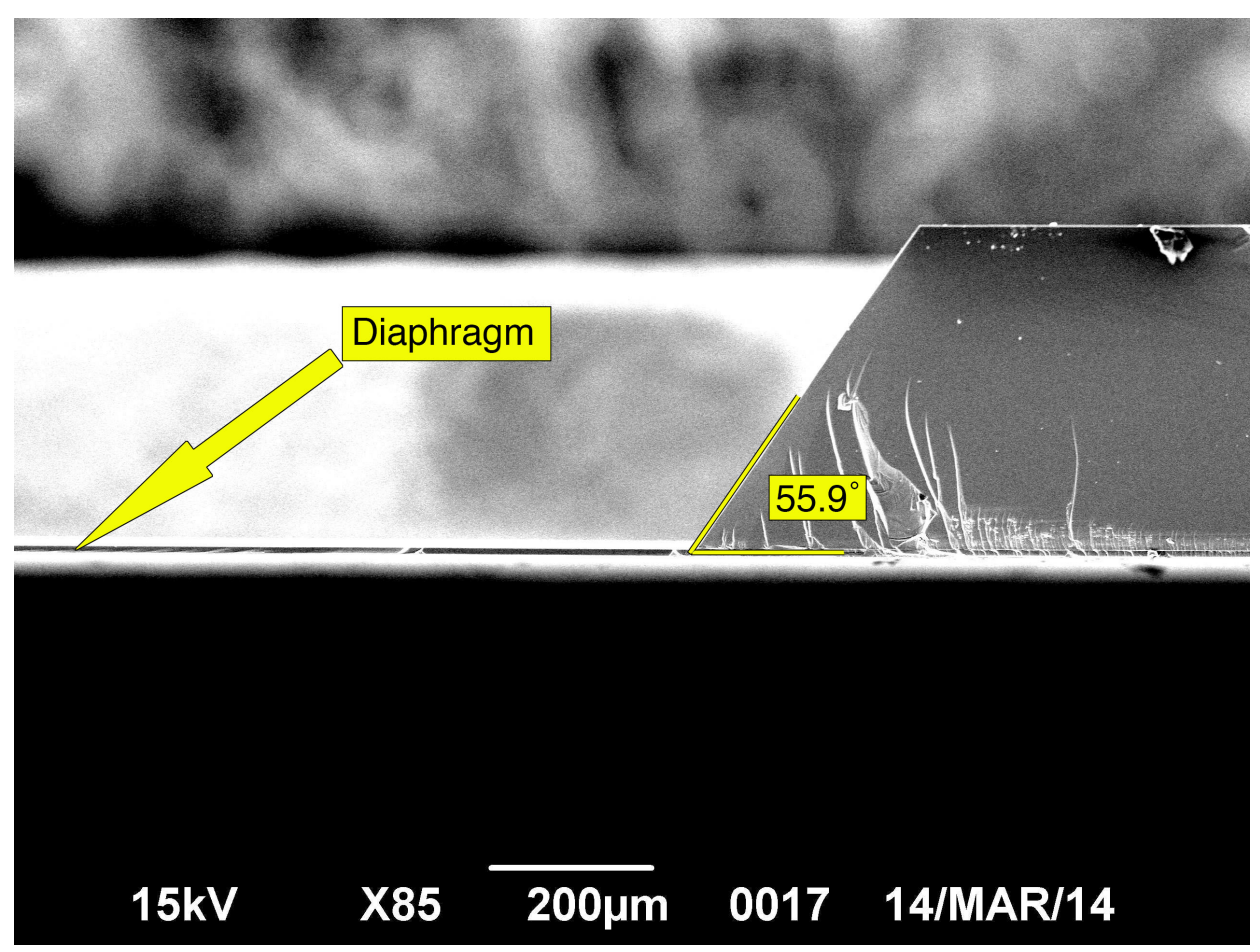

Figure 70: SEM cross-section from process wafer 2 showing the sidewall angle created from the dual doped TMAH etch. The relative thickness of the 10um diaphragm as compared to the 400um handle layer of the SOI wafer is shown. The image was taken at $15 \mathrm{kV}, 85 \mathrm{X}$ magnification, a working distance of $10 \mathrm{~mm}$, and a spot size of 35 .

The sidewall etch characteristics were examined and found to have some nonuniformity, however there did not seem to be any negative effects (Figure 71). 


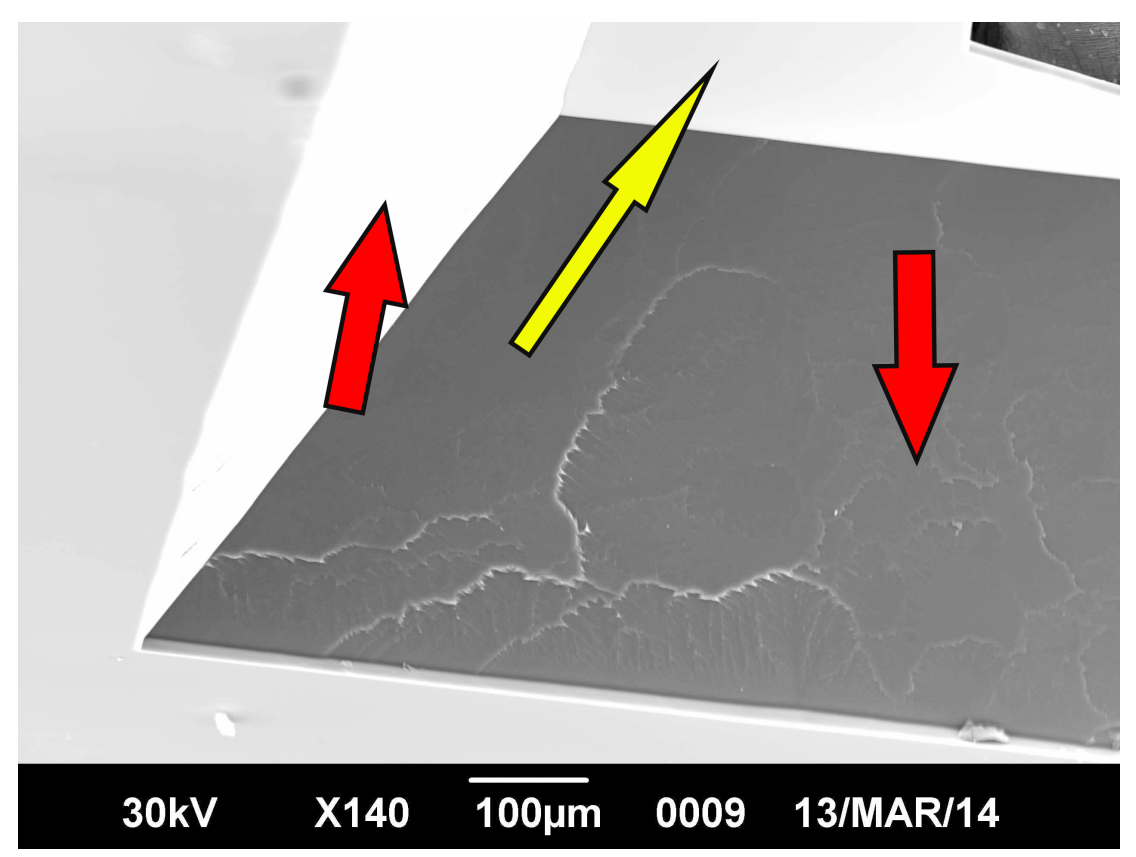

Figure 71: SEM image showing etching non-uniformity on the sidewalls of process wafer 2 , the yellow arrow points to the diaphragm, while the red arrows point to the sidewalls of the diaphragms. The image was taken at $30 \mathrm{kV}, 140 \mathrm{X}$ magnification, a working distance of $21 \mathrm{~mm}$, and a spot size of 77 .

SEM images were also taken from process wafer 1 to confirm the results found from process wafer 2 (Figure 72 to Figure 74). The undercut from the oxide mask was also visible from the process wafer 1 diaphragm imaged (Figure 74). 


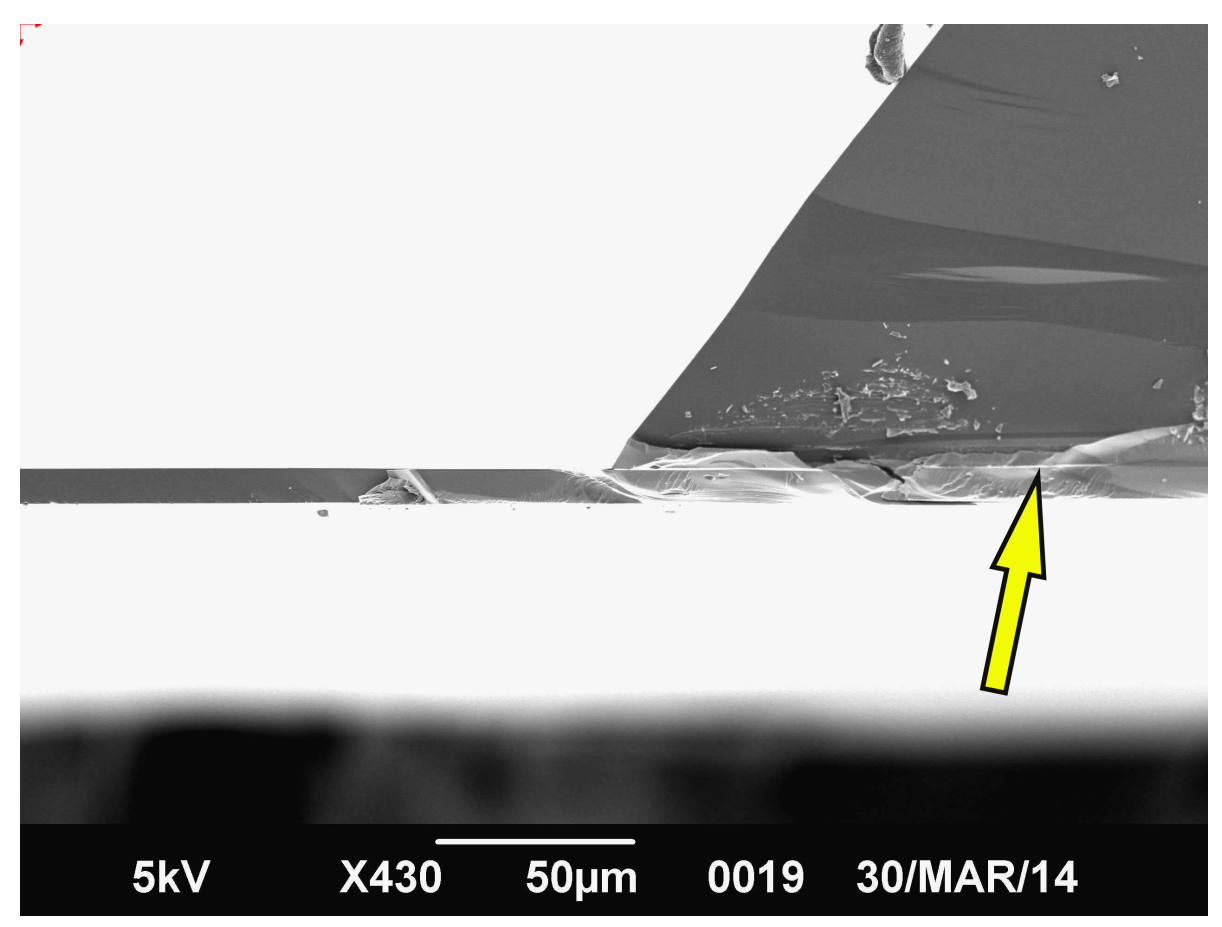

Figure 72: SEM image of process wafer 1 showing a close up of the diaphragm to handle layer interface of the SOI wafer. The arrow points to the buried oxide. The image was taken at $5 \mathrm{kV}$, $430 \mathrm{X}$ magnification, a working distance of $12 \mathrm{~mm}$, and a spot size of 35 .

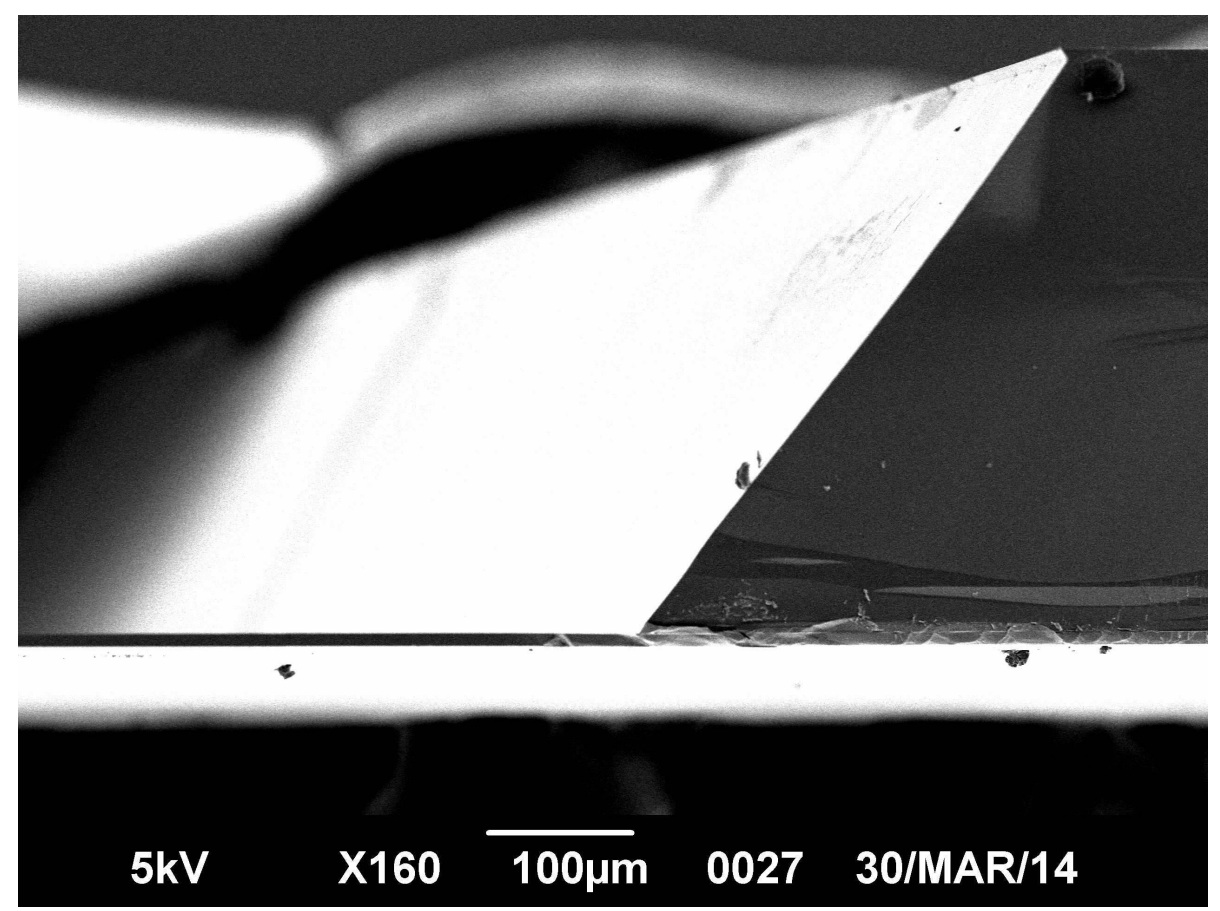

Figure 73: SEM image showing cross section of process wafer 1 . The image was taken at $5 \mathrm{kV}$, 160X magnification, a working distance of $12 \mathrm{~mm}$, and a spot size of 20 . 


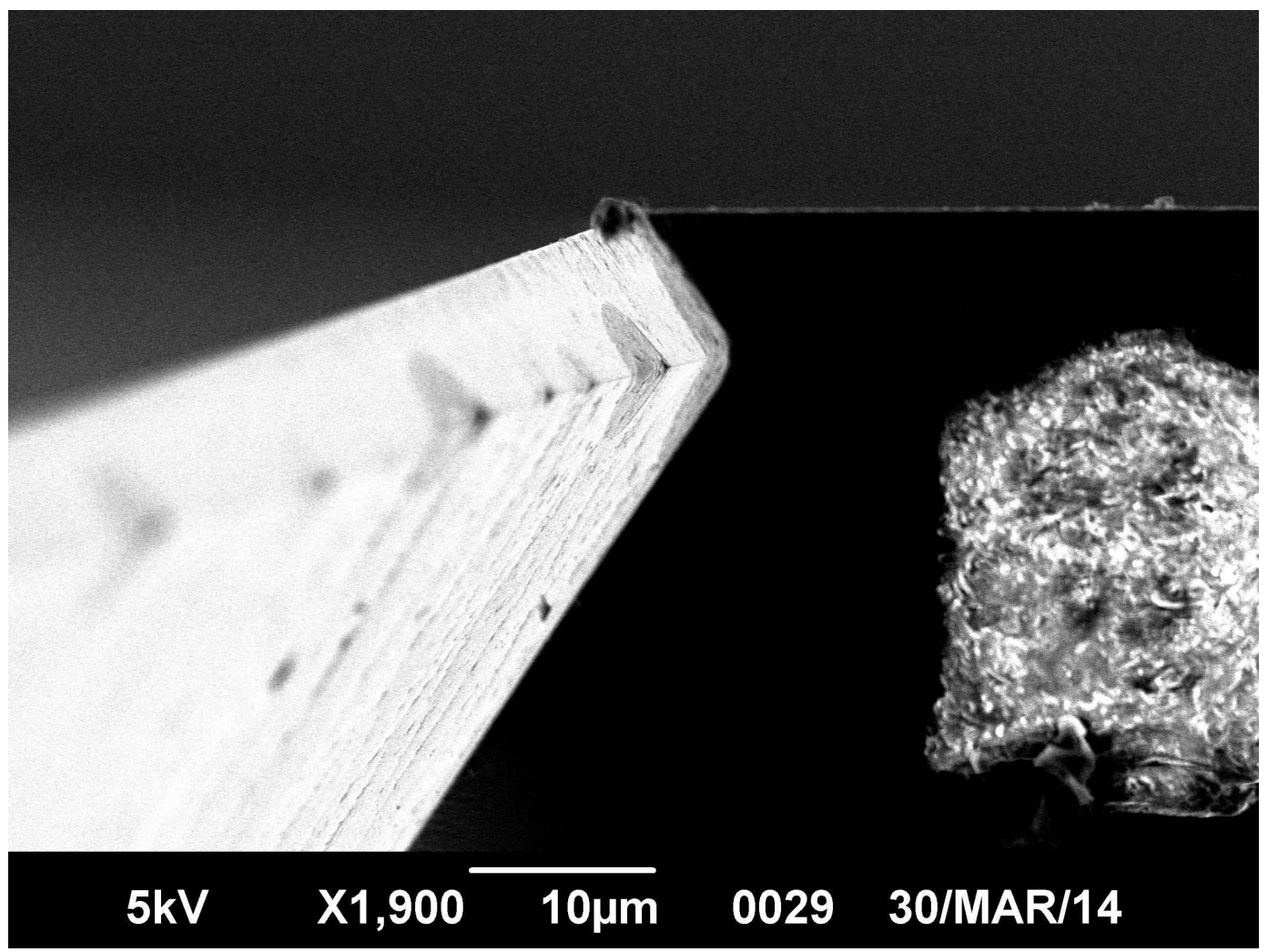

Figure 74: SEM image from process wafer 1 showing undercutting from the dual doped TMAH solution under the oxide mask on the top side of the wafer. The image was taken at $5 \mathrm{kV}, 1900 \mathrm{X}$ magnification, a working distance of $12 \mathrm{~mm}$, and a spot size of 20 .

\subsection{CONCLUSIONS}

Diaphragms of nominal side lengths of $4 \mathrm{~mm}, 5 \mathrm{~mm}$, and $7 \mathrm{~mm}$ were successfully created with a thickness of $10 \mathrm{um}$. Process wafer 1 had a yield of $61 \%$, and process wafer 2 had a yield of $50 \%$, although the pin holes found should not affect the mechanical properties of the diaphragms. The warpage of the largest $(7 \mathrm{~mm})$ diaphragms was shown to be less than $250 \mathrm{~nm}$ after the removal of the buried and thermal oxides. 


\section{DiAPHRAgM TESTING}

\subsection{TEST PREPARATION}

The first process wafer was mounted on a dummy wafer, with Kapton tape placed in between the wafers on the edges. The Kapton tape was measured with the profilometer to be 70um thick, leaving a 70um gap to allow for diaphragm deflection between the two wafers. The second process wafer broke during the final BOE etch, making it necessary to individually mount the diaphragms. 3 diaphragms of each size with no pin holes were selected from the second process wafer. The diaphragms were then broken from the wafer using a glass microscope slide and diamond scribe (Figure 75), and mounted with double sided tape onto glass microscope slides. The tape was placed along the edges of the individual diaphragms so as not to interact with the deflection of the diaphragms during testing. The specified height of the double-sided tape was 101um (3M 4496 Double Coated Polyethylene Foam Tape). The microscope slides were then labeled with the diaphragm size, wafer number, and diaphragm number (Figure 76). 


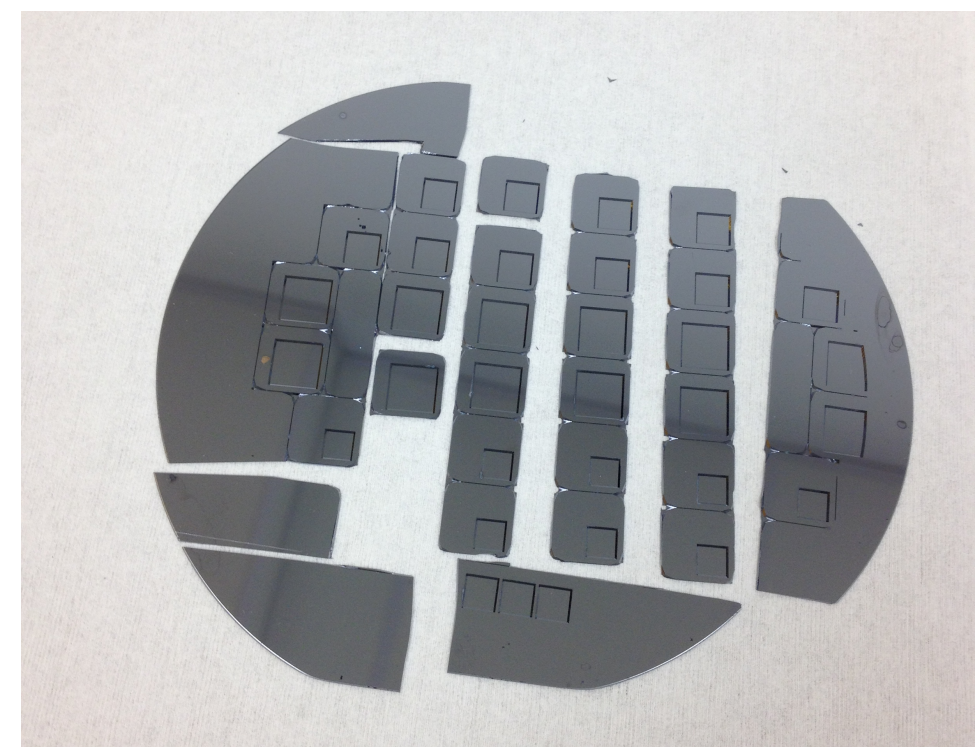

Figure 75: Breaking of the second process wafer to individually mount diaphragms.

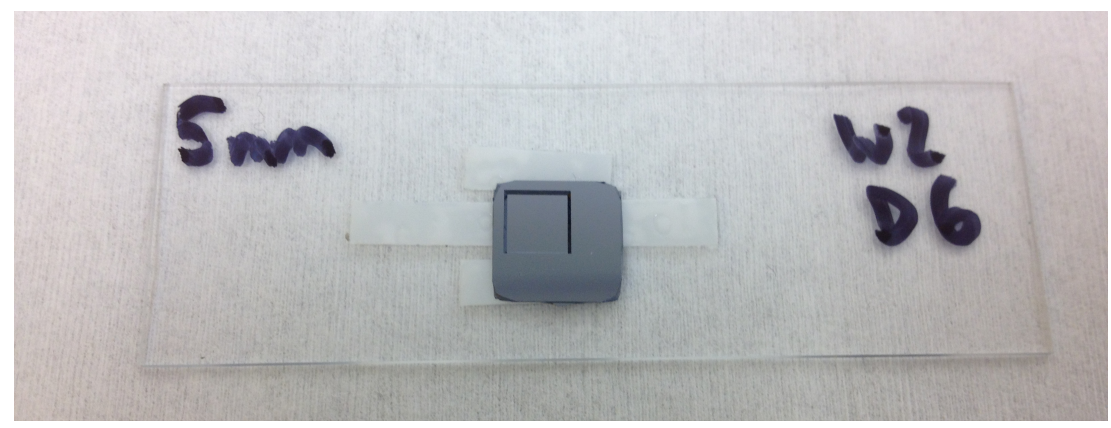

Figure 76: Individually mounted diaphragm from the second process wafer. Double-sided tape holds the diaphragm to a microscope slide. The diaphragm shown is number 6 , a $5 \mathrm{~mm}$ square diaphragm.

\subsection{GAGE R\&R STUDY}

A Gage R\&R study was performed to asses the equipment variability of the system. Because only one operator was used in the study, the appraiser variability was not analyzed. 3 diaphragms of each size $(4,5$, and $7 \mathrm{~mm}$ side length) were measured from both wafers totaling 18 diaphragms. Each diaphragm was tested 3 times, with each test composed of the average of 3 transducer measurements. For each of the test runs, 
a random number generator was used to determine the testing order (Table 17). Prior to testing, all equipment was turned on. The Keithley 2400 was turned on with the output on and "Volts" selected. The amplifier was adjusted to produce a transducer output of approximately $-.15 \mathrm{~V}$. The test program was initiated (which turned off the Keithley 2400 output). The procedure for each test was as follows:

1) Place the diaphragm on the linear stage

2) Align the vertical diaphragm edge with the cross hairs (to eliminate any theta error in the alignment)

3) Move the $x$ and $y$ stages to align the center of the cross hairs with the center of the diaphragm

4) Click the "Move to Test Pos" button, the center of the diaphragm was translated on the $\mathrm{X} \& \mathrm{Y}$ stages beneath the transducer probe (Figure 77)

5) Lower the $Z$ stage in 100 en steps until the amplifier indicator LED changes from negative to positive (indicating that contact has been made with the sample) (Figure 78)

6) Raise the $Z$ stage 200 en steps to bring the transducer tip off the sample and to account for the backlash of the linear stage.

7) Begin the test, with $1000 \mathrm{~ms}$ between each sample, with a displacement step size of 1 en.

8) Once the appropriate maximum voltage is achieved, the transducer was raised off the sample, and the measurement was run again for a total of three measurements

9) After the measurements were taken, the transducer was raised above the sample to a height above the edge of the diaphragm and the sample was 
moved back to the loading position under the camera cross hairs by pressing the "Backtrack" button

10) Repeat for the next sample

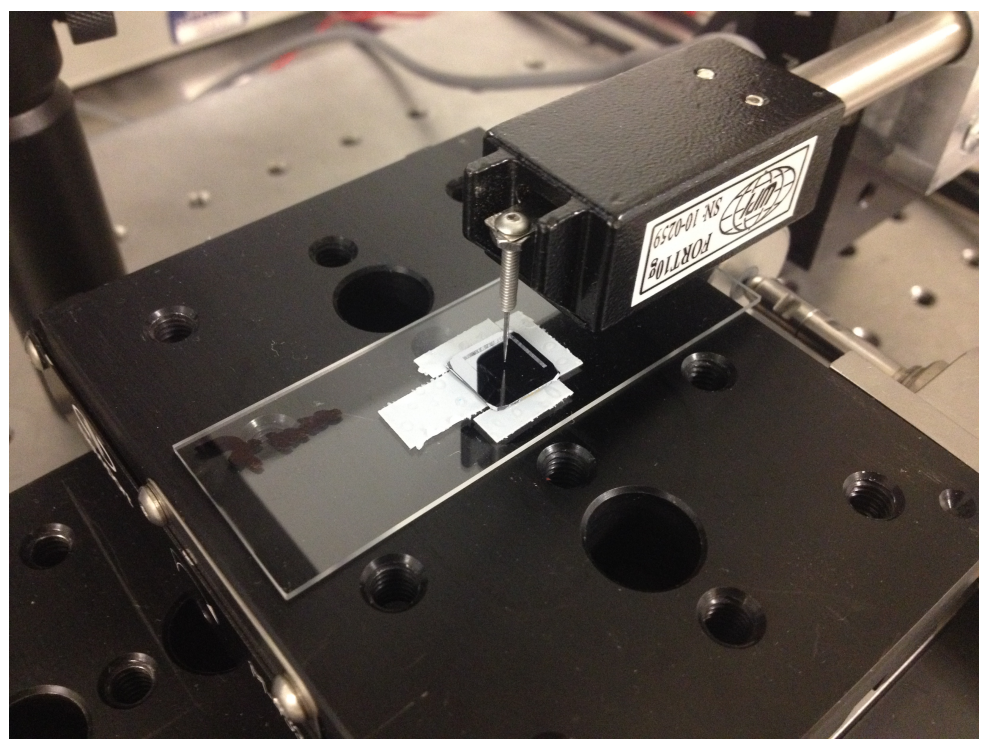

Figure 77: Placement of test diaphragm beneath probe tip during Gage R\&R study.

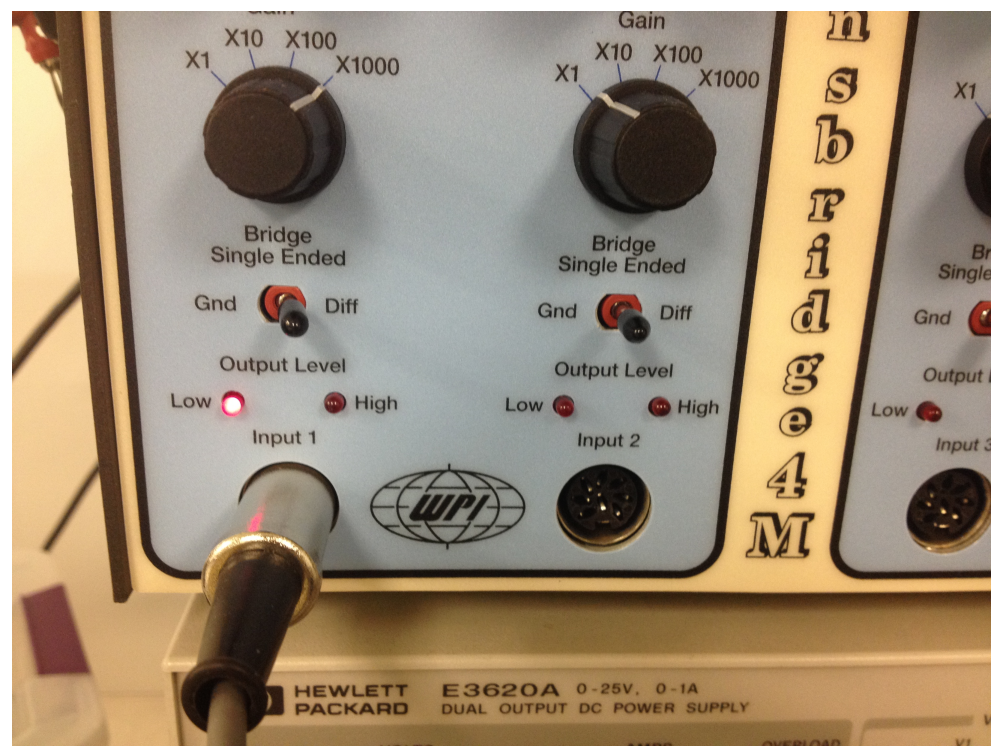

Figure 78: Amplifier used in Gage R\&R study, note the Output Level is currently below zero volts as indicated by the "Low" red LED. 
Table 17: Gage R\&R testing information for process wafer 1 and 2.

\begin{tabular}{|r|r|r|r|r|r|}
\hline Wafer No. & Dia. Size & Dia. No. & Run 1 Order & Run 2 Order & Run 3 Order \\
\hline 1 & 4 & 29 & 13 & 10 & 10 \\
\hline 1 & 4 & 30 & 12 & 17 & 11 \\
\hline 1 & 4 & 31 & 8 & 12 & 3 \\
\hline 1 & 5 & 11 & 16 & 16 & 6 \\
\hline 1 & 5 & 12 & 17 & 6 & 13 \\
\hline 1 & 5 & 13 & 6 & 4 & 12 \\
\hline 1 & 7 & 19 & 3 & 18 & 17 \\
\hline 1 & 7 & 23 & 5 & 5 & 1 \\
\hline 1 & 7 & 24 & 11 & 2 & 9 \\
\hline 2 & 4 & 29 & 4 & 3 & 18 \\
\hline 2 & 4 & 34 & 1 & 7 & 5 \\
\hline 2 & 4 & 35 & 14 & 13 & 15 \\
\hline 2 & 5 & 5 & 9 & 11 & 4 \\
\hline 2 & 5 & 6 & 2 & 8 & 16 \\
\hline 2 & 5 & 11 & 18 & 9 & 2 \\
\hline 2 & 7 & 17 & 10 & 1 & 8 \\
\hline 2 & 7 & 23 & 7 & 15 & 7 \\
\hline 2 & 7 & 24 & 15 & 14 & 14 \\
\hline & & & & & \\
\hline
\end{tabular}

The results of the Gage R\&R study were plotted, showing discernable regions of stiffness for each diaphragm (Figure 79). 


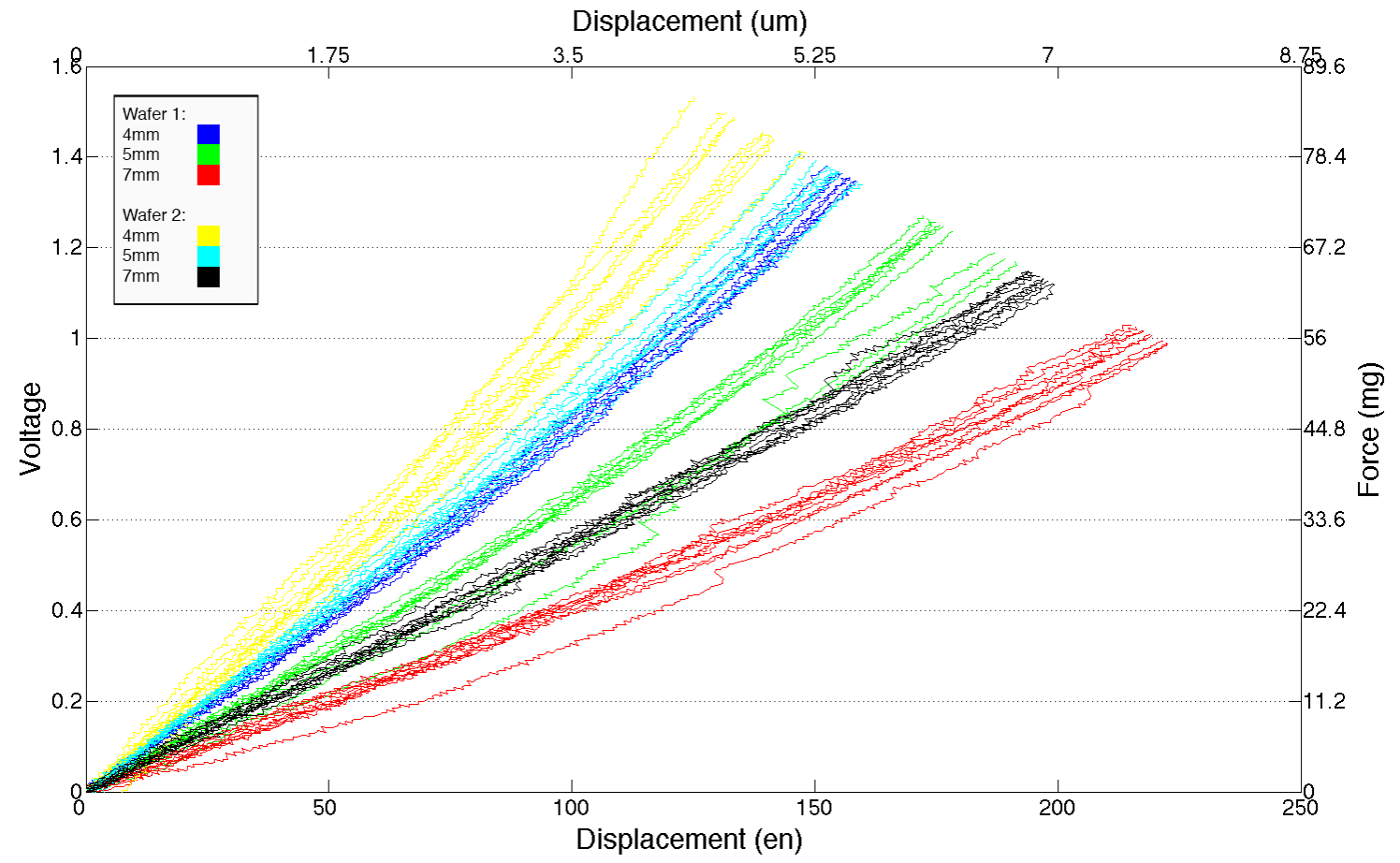

Figure 79: Gage R\&R results from both process wafers and 3 different diaphragm sizes.

The results were separated by wafer to more easily visualize the results (Figure

\section{0 and Figure 81)}

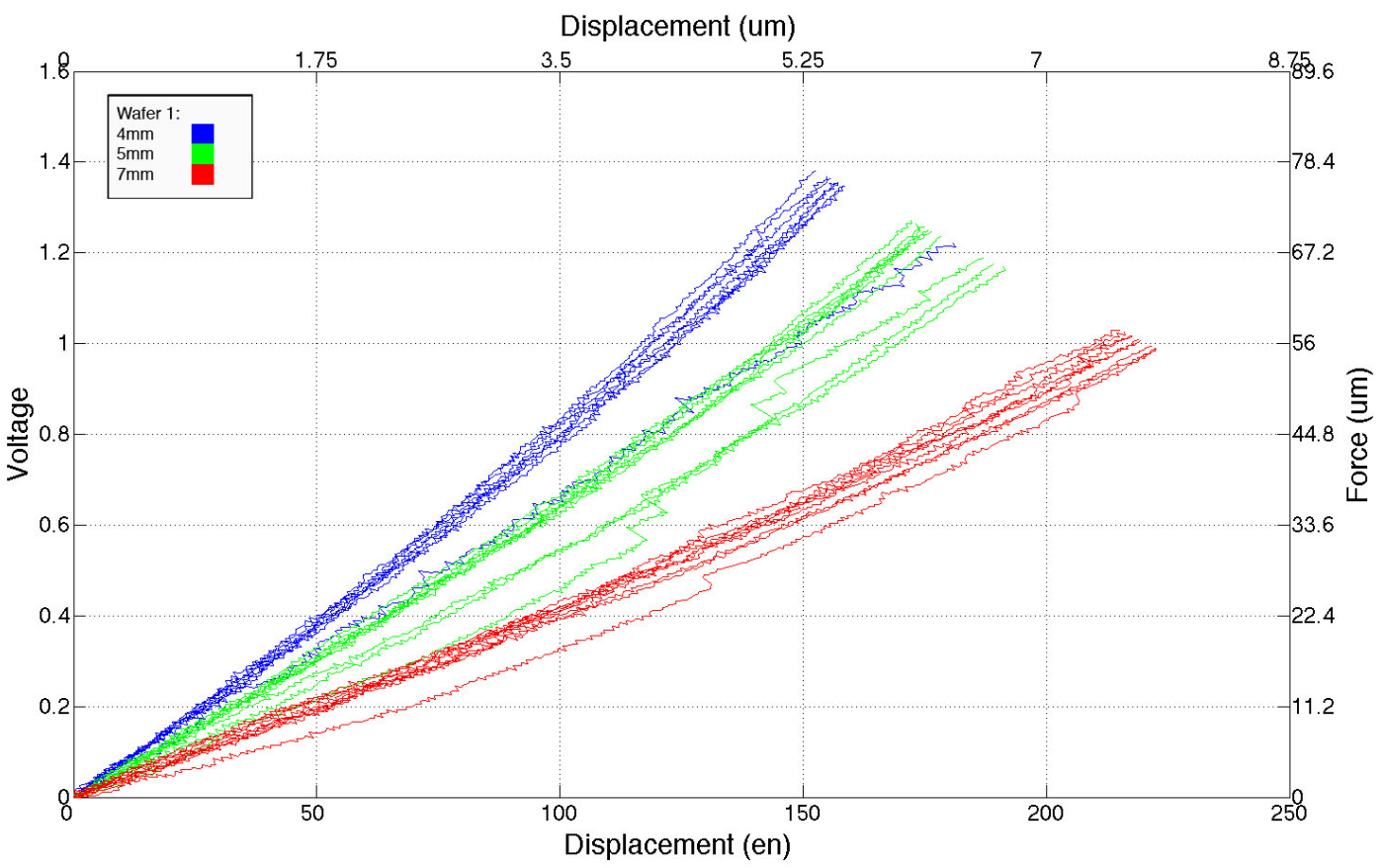

Figure 80: Gage R\&R results for process wafer 1. 


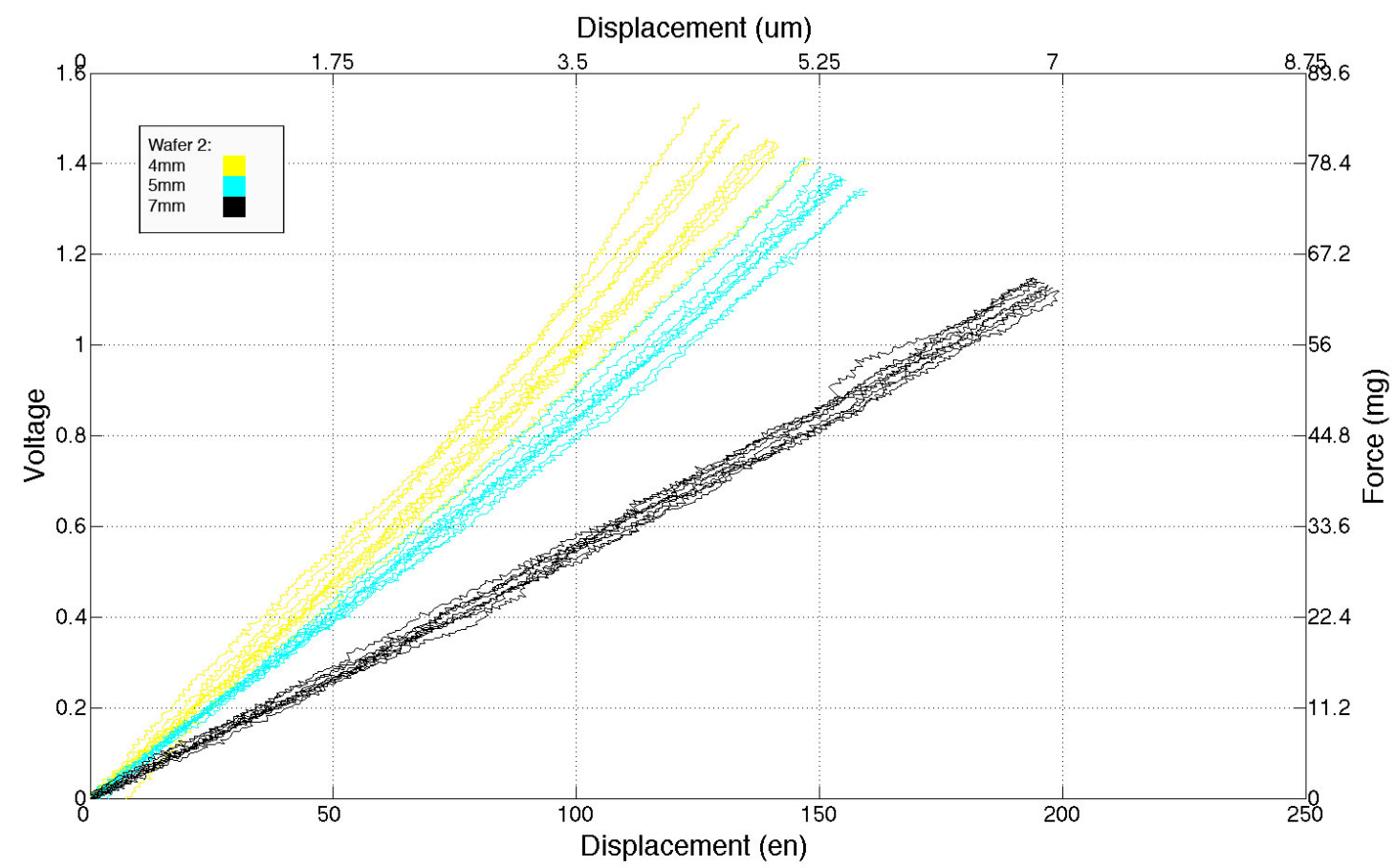

Figure 81: Gage R\&R results for process wafer 2.

Gage R\&R information was collected at 50 and 100 en units of displacement (Table 18 and Table 19), consisting of Xbar (the average of the trial average for each diaphragm size) and Rbar (the average of the range for each diaphragm size). The equipment variability (Ev) was calculated as the average of all of the Rbar values multiplied by a constant (based on the number of trials, in this case .5908$)^{24}$. The part variability $(\mathrm{Pv})$ was the difference between the maximum and minimum Xbar values. For the Gage R\&R study to be successful, the Ev must be below the Pv, signifying that the different parts are discernable. ${ }^{25}$

Table 18: Gage R\&R results at 50en units of displacement.

\begin{tabular}{|r|r|r|r|}
\hline Wafer No. & \multicolumn{1}{l|}{ Diaphragm Size } & \multicolumn{1}{l|}{ Xbar } & \multicolumn{1}{l|}{ Rbar } \\
\hline 1 & 4 & 0.3713 & 0.0218 \\
\hline 1 & 5 & 0.2858 & 0.0356 \\
\hline 1 & 7 & 0.1957 & 0.0319 \\
\hline 2 & 4 & 0.4861 & 0.0471 \\
\hline 2 & 5 & 0.4025 & 0.0302 \\
\hline 2 & 7 & 0.2695 & 0.0220 \\
\hline
\end{tabular}


Table 19: Gage R\&R results at 100en units of displacement.

\begin{tabular}{|r|r|r|r|}
\hline Wafer No. & \multicolumn{1}{l|}{ Diaphragm Size } & \multicolumn{1}{l|}{ Xbar } & \multicolumn{1}{l|}{ Rbar } \\
\hline 1 & 4 & 0.7787 & 0.0768 \\
\hline 1 & 5 & 0.6019 & 0.0461 \\
\hline 1 & 7 & 0.4003 & 0.0374 \\
\hline 2 & 4 & 1.0203 & 0.0813 \\
\hline 2 & 5 & 0.8406 & 0.0650 \\
\hline 2 & 7 & 0.5481 & 0.0356 \\
\hline
\end{tabular}

The Ev for 50 and 100 en units of displacement was found to be well below the $\mathrm{Pv}$ at each displacement unit, signifying that the individual diaphragm sizes were resolvable though the test equipment.

Table 20: Ev and Pv values for test values of 50 and 100en units of displacement.

\begin{tabular}{|r|r|r|}
\hline Test Displacement & Equipment Variation (Ev) & Part Variation (Pv) \\
\hline $50 \mathrm{en}$ & 0.0186 & 0.290 \\
\hline 100en & 0.0337 & 0.620 \\
\hline
\end{tabular}

To confirm these results, a one-way ANOVA test was performed on each wafer at 50 and 100en units of displacement to determine the statistical significance at $\alpha=0.05$ of the voltage output from the transducer testing each diaphragm size. Box plots of the voltage output produced from the transducer for each diaphragm size were created (Figure 82:Figure 85) as well as summary tables of the results (Table 21:Table 32). 


\section{Summary Statistics for ANOVA test for Wafer 1 at 50en Units of Displacement:}

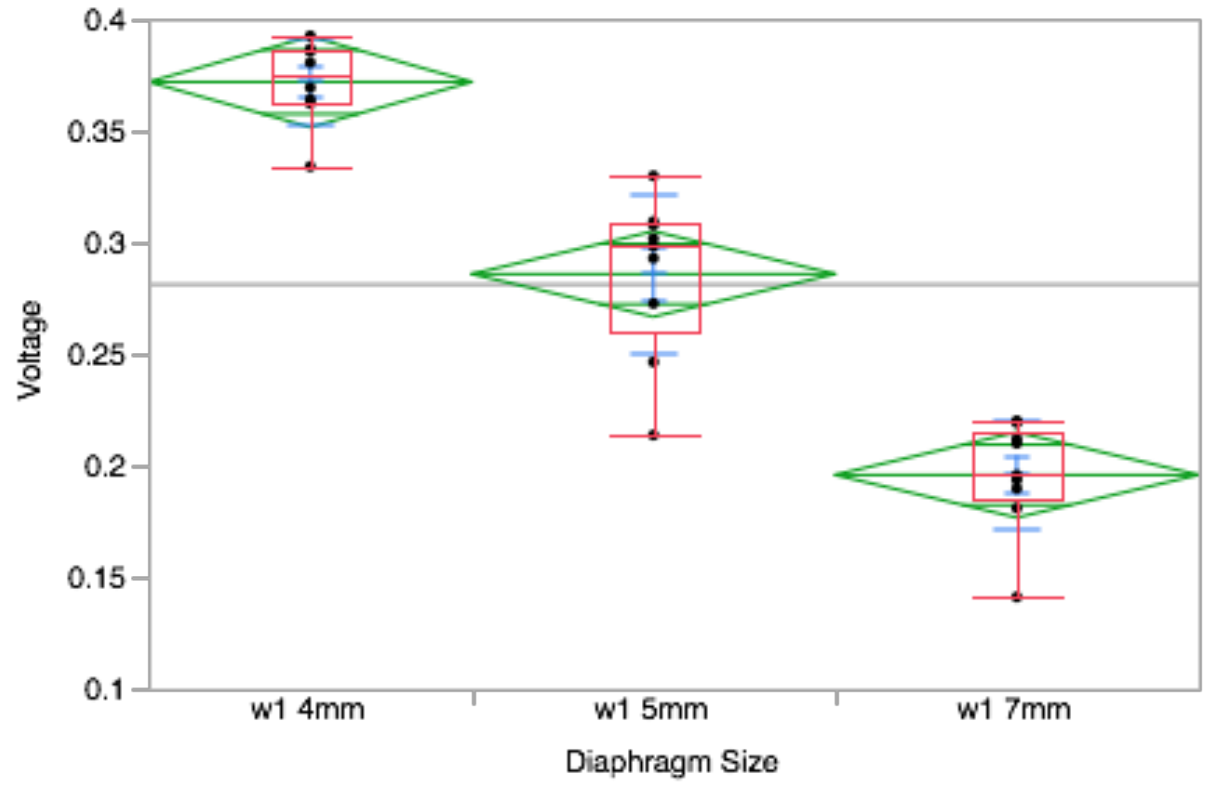

Figure 82: One way analysis of voltage by diaphragm size for process wafer 1 at 50 en units of displacement.

Table 21: One way ANOVA summary of fit, wafer 1 at $50 \mathrm{en}$.

\begin{tabular}{|l|r|}
\hline Rsquare & 0.881389 \\
\hline Adj Rsquare & 0.871075 \\
\hline Root Mean Square Error & 0.027766 \\
\hline Mean of Response & 0.281131 \\
\hline Observations (or Sum Wgts) & 26 \\
\hline
\end{tabular}

Table 22: Analysis of variance, wafer 1 at $50 \mathrm{en}$.

\begin{tabular}{|l|r|r|r|r|r|}
\hline Source & DF & $\begin{array}{r}\text { Sum of } \\
\text { Squares }\end{array}$ & Mean Square & F Ratio & Prob > F \\
\hline Diaphragm Size & 2 & 0.13176331 & 0.065882 & 85.4556 & $<.0001^{*}$ \\
\hline Error & 23 & 0.01773177 & 0.000771 & & \\
\hline C. Total & 25 & 0.14949508 & & & \\
\hline
\end{tabular}

Table 23: Means for one way ANOVA, wafer 1 at $50 \mathrm{en}$.

\begin{tabular}{|l|r|r|r|r|r|}
\hline Level & Number & Mean & Std Error & Lower 95\% & Upper 95\% \\
\hline w1 4mm & 8 & 0.371925 & 0.00982 & 0.35162 & 0.39223 \\
\hline w1 $5 \mathrm{~mm}$ & 9 & 0.285811 & 0.00926 & 0.26667 & 0.30496 \\
\hline w1 $7 \mathrm{~mm}$ & 9 & 0.195744 & 0.00926 & 0.17660 & 0.21489 \\
\hline
\end{tabular}




\section{Summary Statistics for ANOVA test for Wafer 2 at 50en Units of Displacement:}

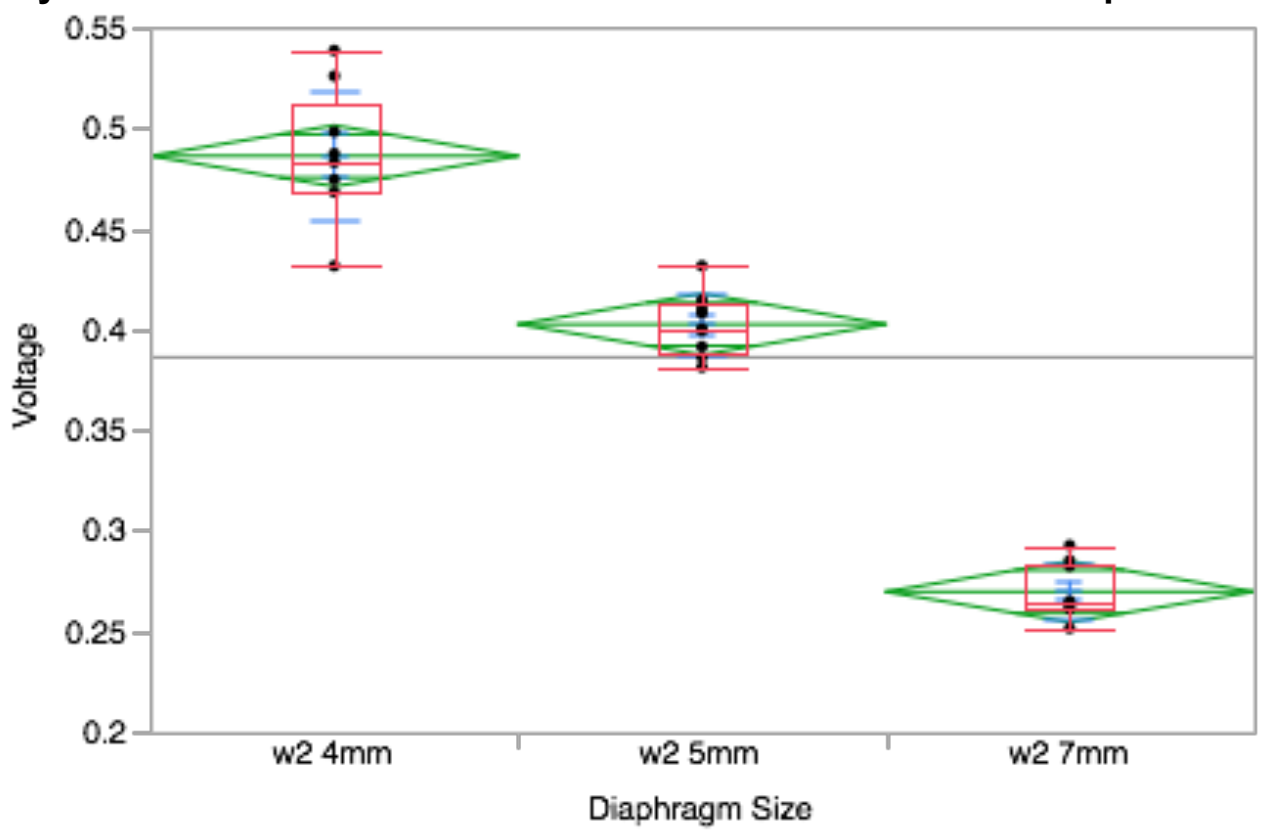

Figure 83: One way analysis of voltage by diaphragm size for process wafer 2 at 50en.

Table 24: One way ANOVA summary of fit, wafer 2 at $50 \mathrm{en}$.

\begin{tabular}{|l|r|}
\hline Rsquare & 0.948415 \\
\hline Adj Rsquare & 0.944116 \\
\hline Root Mean Square Error & 0.022062 \\
\hline Mean of Response & 0.386056 \\
\hline Observations (or Sum Wgts) & 27 \\
\hline
\end{tabular}

Table 25: Analysis of variance, wafer 2 at $50 \mathrm{en}$.

\begin{tabular}{|l|r|r|r|r|r|}
\hline Source & DF & $\begin{array}{r}\text { Sum of } \\
\text { Squares }\end{array}$ & Mean Square & F Ratio & Prob > F \\
\hline Diaphragm Size & 2 & 0.21476083 & 0.107380 & 220.6250 & $<.0001^{*}$ \\
\hline Error & 24 & 0.01168104 & 0.000487 & & \\
\hline C. Total & 26 & 0.22644187 & & & \\
\hline
\end{tabular}

Table 26: Means for one way ANOVA, wafer 2 at $50 \mathrm{en}$.

\begin{tabular}{|l|r|r|r|r|r|}
\hline Level & Number & Mean & Std Error & Lower 95\% & Upper 95\% \\
\hline w2 $4 \mathrm{~mm}$ & 9 & 0.486144 & 0.00735 & 0.47097 & 0.50132 \\
\hline w2 $5 \mathrm{~mm}$ & 9 & 0.402478 & 0.00735 & 0.38730 & 0.41766 \\
\hline w2 $7 \mathrm{~mm}$ & 9 & 0.269544 & 0.00735 & 0.25437 & 0.28472 \\
\hline
\end{tabular}




\section{Summary Statistics for ANOVA test for Wafer 1 at 100en Units of Displacement:}

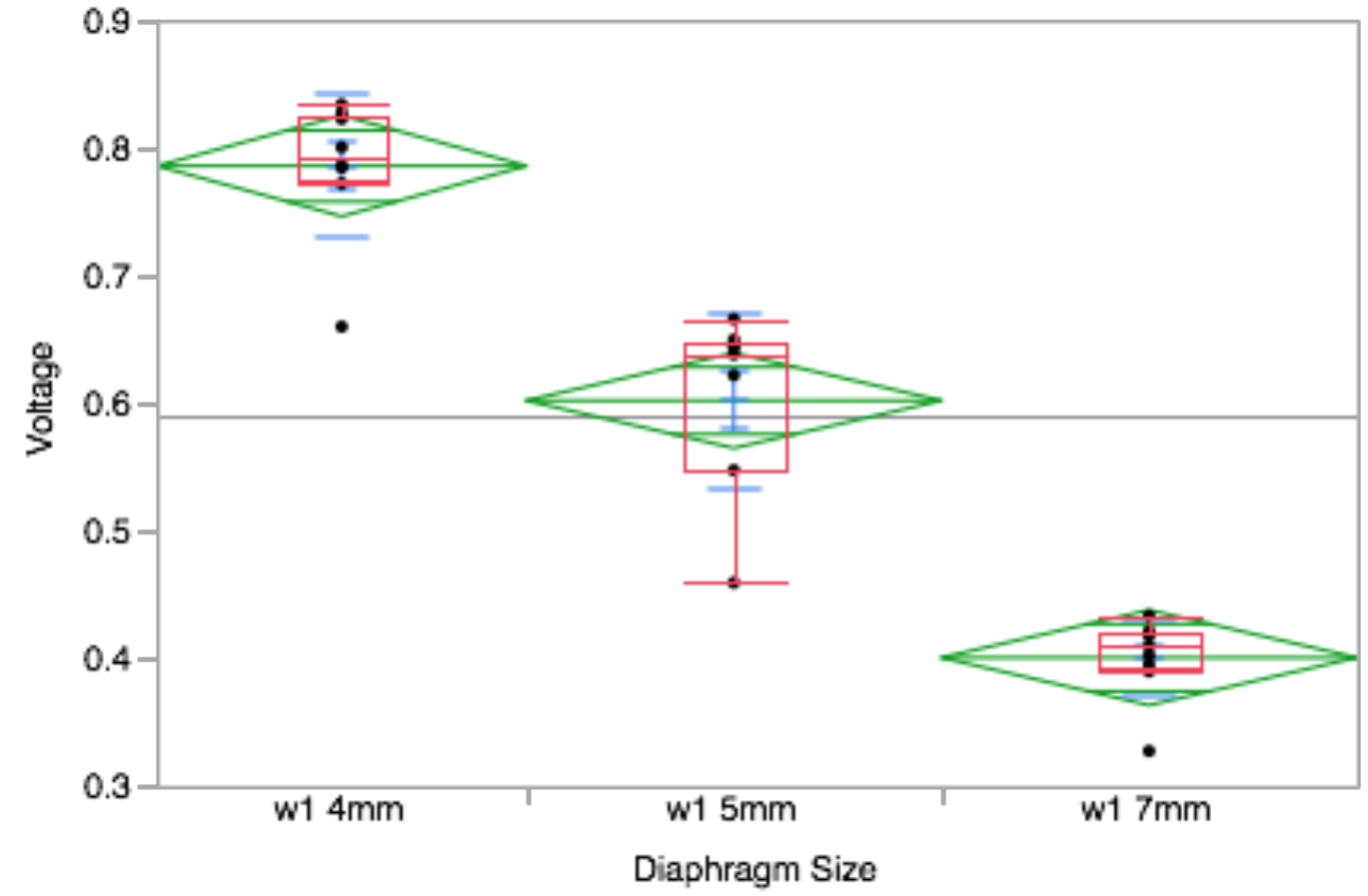

Figure 84: One way analysis of voltage by diaphragm size for process wafer 1 at $100 \mathrm{en}$.

Table 27: One way ANOVA summary of fit, wafer 1 at $100 \mathrm{en}$.

\begin{tabular}{|l|r|}
\hline Rsquare & 0.903851 \\
\hline Adj Rsquare & 0.89549 \\
\hline Root Mean Square Error & 0.05408 \\
\hline Mean of Response & 0.588715 \\
\hline Observations (or Sum Wgts) & 26 \\
\hline
\end{tabular}

Analysis of Variance

Table 28: Analysis of variance, wafer 1 at $100 \mathrm{en}$

\begin{tabular}{|l|r|r|r|r|r|}
\hline Source & DF & $\begin{array}{r}\text { Sum of } \\
\text { Squares }\end{array}$ & Mean Square & F Ratio & Prob > F \\
\hline Diaphragm Size & 2 & 0.63234626 & 0.316173 & 108.1059 & $<.0001^{*}$ \\
\hline Error & 23 & 0.06726721 & 0.002925 & & \\
\hline C. Total & 25 & 0.69961347 & & & \\
\hline
\end{tabular}

\section{Means for Oneway Anova}

Table 29: Means for one way ANOVA, wafer 1 at $100 \mathrm{en}$.

\begin{tabular}{|l|r|r|r|r|r|}
\hline Level & Number & Mean & Std Error & Lower 95\% & Upper 95\% \\
\hline w1 4mm & 8 & 0.785925 & 0.01912 & 0.74637 & 0.82548 \\
\hline w1 $5 \mathrm{~mm}$ & 9 & 0.601878 & 0.01803 & 0.56459 & 0.63917 \\
\hline w1 $7 \mathrm{~mm}$ & 9 & 0.400256 & 0.01803 & 0.36296 & 0.43755 \\
\hline
\end{tabular}




\section{Summary Statistics for ANOVA test for Wafer 2 at 100en Units of Displacement:}

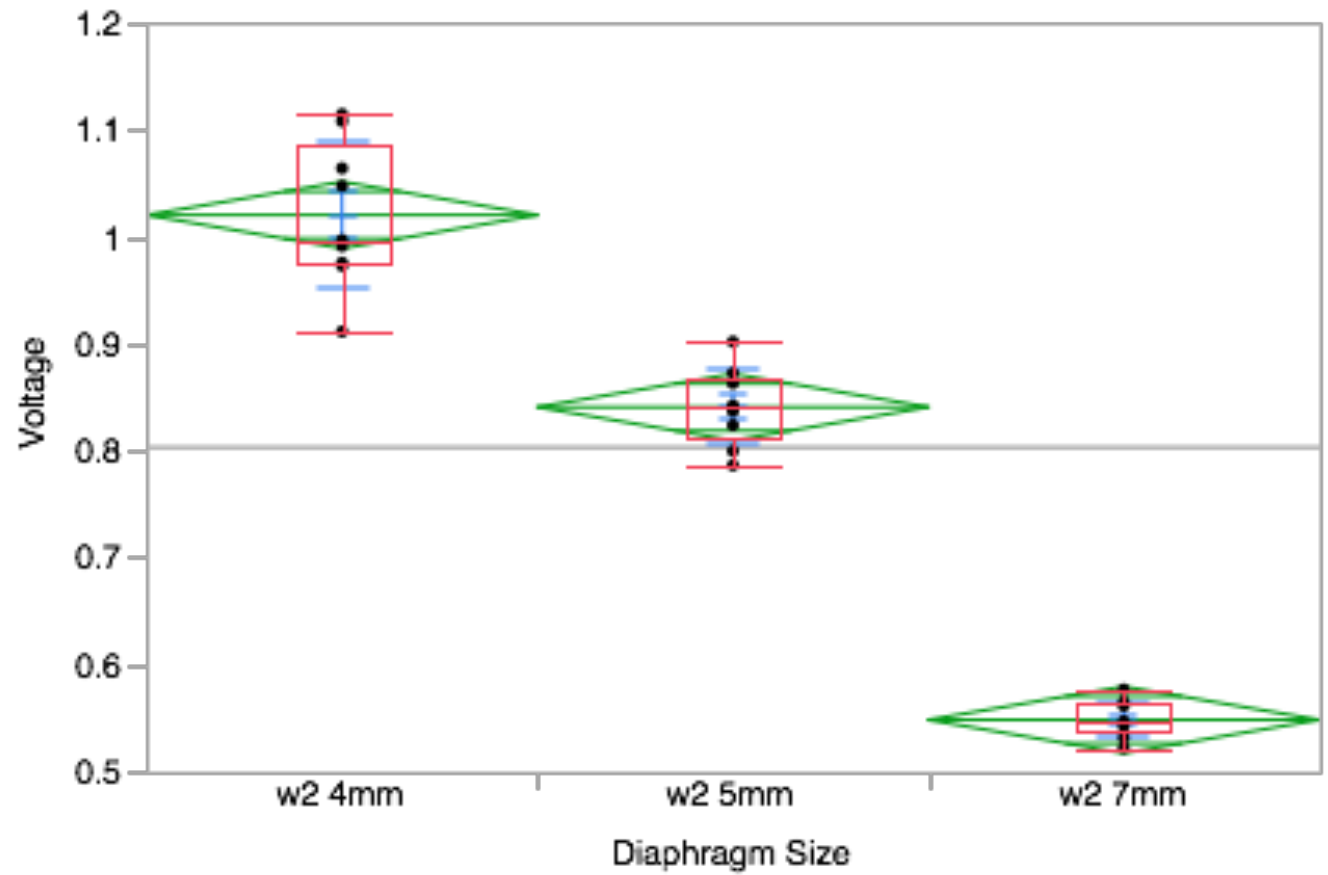

Figure 85: One way analysis of voltage by diaphragm size for process wafer 2 at $100 \mathrm{en}$.

Table 30: One way ANOVA summary of fit, wafer 2 at $100 \mathrm{en}$.

\begin{tabular}{|l|r|}
\hline Rsquare & 0.954015 \\
\hline Adj Rsquare & 0.950183 \\
\hline Root Mean Square Error & 0.045315 \\
\hline Mean of Response & 0.802989 \\
\hline Observations (or Sum Wgts) & 27 \\
\hline
\end{tabular}

Table 31: Analysis of variance, wafer 2 at $100 \mathrm{en}$.

\begin{tabular}{|l|r|r|r|r|r|}
\hline Source & DF & $\begin{array}{r}\text { Sum of } \\
\text { Squares }\end{array}$ & Mean Square & F Ratio & Prob > F \\
\hline Diaphragm Size & 2 & 1.0224502 & 0.511225 & 248.9554 & $<.0001^{*}$ \\
\hline Error & 24 & 0.0492835 & 0.002053 & & \\
\hline C. Total & 26 & 1.0717337 & & & \\
\hline
\end{tabular}

Table 32: Means for one way ANOVA, wafer 2 at 100 en.

\begin{tabular}{|l|r|r|r|r|r|}
\hline Level & Number & Mean & Std Error & Lower 95\% & Upper 95\% \\
\hline w2 4mm & 9 & 1.02027 & 0.01511 & 0.98909 & 1.0514 \\
\hline w2 $5 \mathrm{~mm}$ & 9 & 0.84062 & 0.01511 & 0.80945 & 0.8718 \\
\hline w2 $7 \mathrm{~mm}$ & 9 & 0.54808 & 0.01511 & 0.51690 & 0.5793 \\
\hline
\end{tabular}


As shown in the preceding pages, all of the ANOVA results indicate significant differences in the means of the voltage produced at each diaphragm size with a probability of the F statistic less than .0001 .

\subsection{COMPARISON OF TEST RESULTS TO MODELING}

The linear displacement diaphragm model (Eq. 17) used for the lithography mask sizing was compared to the actual results obtained from the Gage R\&R study. First, the same model was applied, with an E=170Gpa, and thickness of $10 \mathrm{um}$. The side lengths for each wafer were determined using the minimum values found in Table 16 . The difference between the model and Gage R\&R studies can be seen in Table 33, Figure 86 and Figure 87.

$$
y_{m}=k_{1} \frac{P b^{2}}{E t^{3}}
$$

Table 33: Force Values at Select Displacement Values.

\begin{tabular}{|l|l|l|}
\hline & \multicolumn{2}{|c|}{ Force at Select Displacement Values } \\
\hline Sample & $\begin{array}{l}\text { 1.75 um (\% change } \\
\text { from theoretical) }\end{array}$ & $\begin{array}{l}3.5 \\
\text { from um (\% change }\end{array}$ \\
\hline Wafer 1, 4mm & $20.49 \mathrm{mg} \mathrm{(33 \% )}$ & $43.30 \mathrm{mg} \mathrm{(30 \% )}$ \\
\hline Wafer 1, 5mm & $15.75 \mathrm{mg} \mathrm{(20 \% )}$ & $33.16 \mathrm{mg} \mathrm{(16 \% )}$ \\
\hline Wafer 1, 7mm & $10.78 \mathrm{mg} \mathrm{(7 \% )}$ & $22.04 \mathrm{mg} \mathrm{(9 \% )}$ \\
\hline Wafer 2, 4mm & $26.78 \mathrm{mg} \mathrm{(13 \% )}$ & $56.22 \mathrm{mg} \mathrm{(9 \% )}$ \\
\hline Wafer 2, 5mm & $22.17 \mathrm{mg} \mathrm{(12 \% )}$ & $46.31 \mathrm{mg} \mathrm{(17 \% )}$ \\
\hline Wafer 2, 7mm & $14.82 \mathrm{mg} \mathrm{(46 \% )}$ & $30.19 \mathrm{mg} \mathrm{(49 \% )}$ \\
\hline Theoretical 4mm & $30.92 \mathrm{mg}$ & $61.84 \mathrm{mg}$ \\
\hline Theoretical $\mathbf{5 m m}$ & $19.79 \mathrm{mg}$ & $39.58 \mathrm{mg}$ \\
\hline Theoretical 7mm & $10.09 \mathrm{mg}$ & $20.19 \mathrm{mg}$ \\
\hline
\end{tabular}




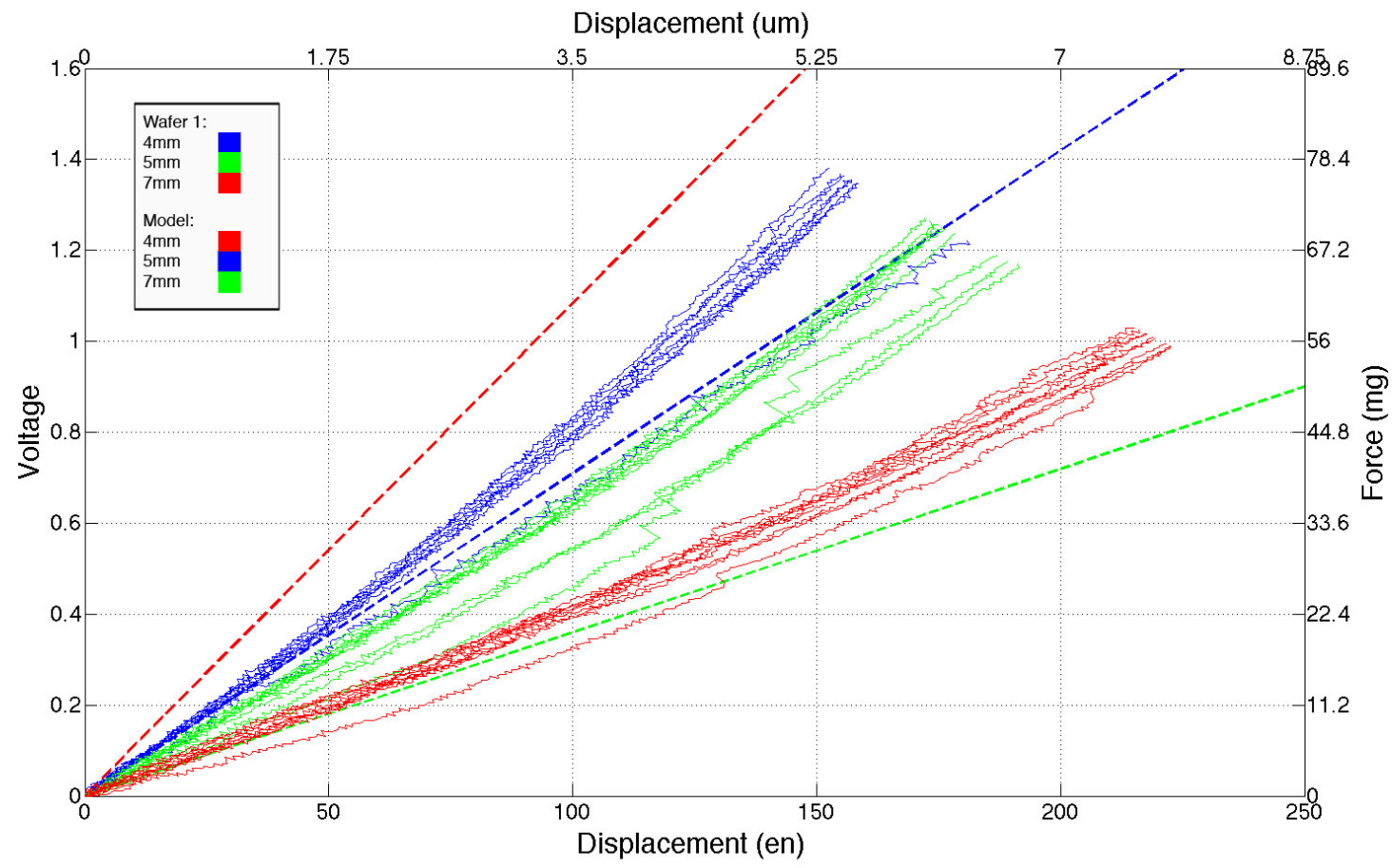

Figure 86: Process wafer 1 Gage R\&R results as compared to theoretical model.

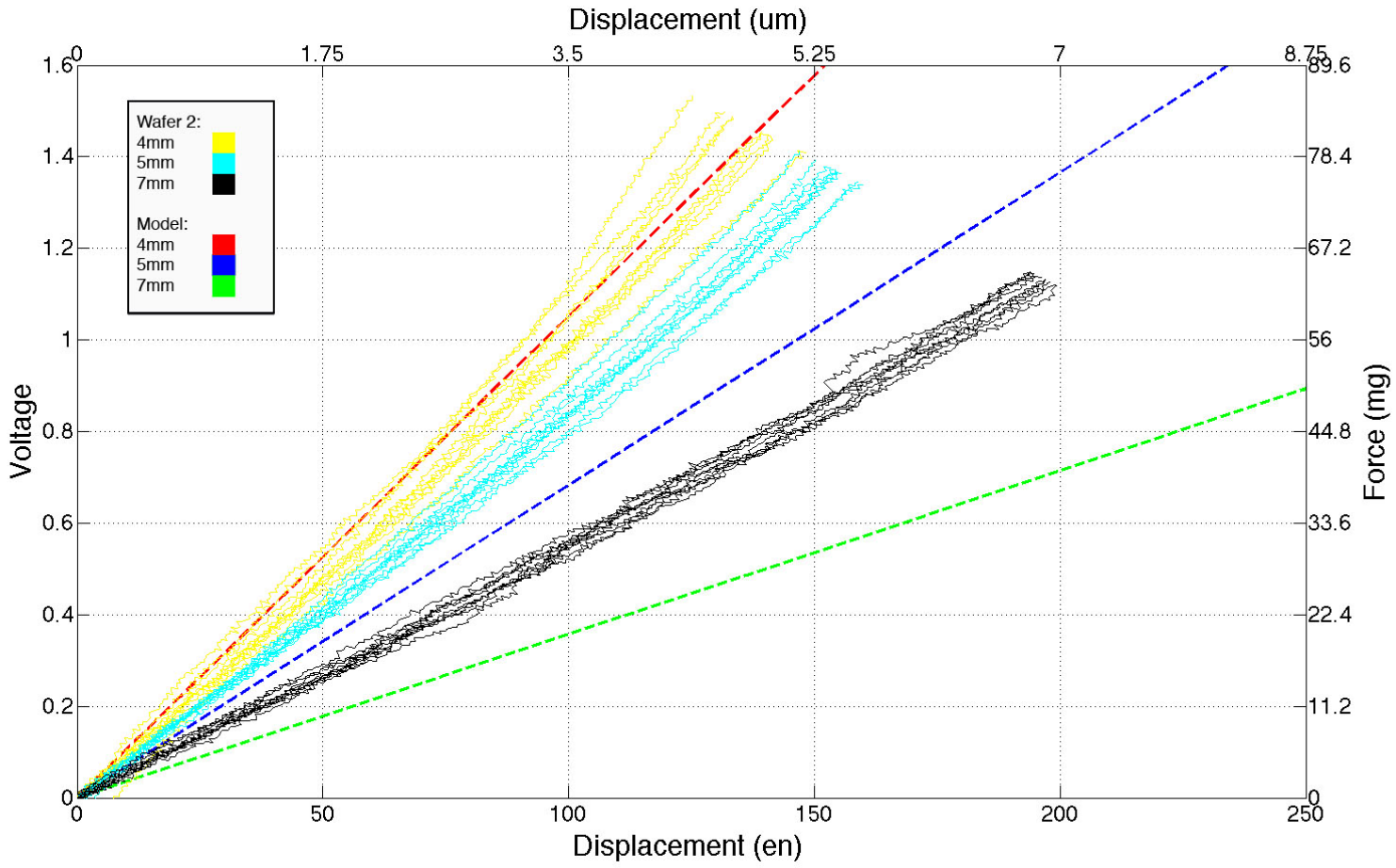

Figure 87: Process wafer 2 results from Gage R\&R study as compared to theoretical model. 
The model is close to the actual results, although there is more spread in the model than there is in the observed results from the Gage R\&R study. This could be due to edge effects from the etching process or effects from the size of the probe tip used to analyze the diaphragms. To compensate for the difference between the model and observed results, the slopes of the model and observed results were plotted and a quadratic equation was derived from the difference in slopes. The compensation equation takes an input of side length (in $\mathrm{mm}$ ) and outputs a multiplying factor to the theoretical model to decrease the spread between diaphragm sizes.

$$
\text { multiplier }=\frac{1}{-0.0197 \text { Sidelengt } h^{2}+0.341 \text { Sidelength }-0.311}
$$

Eq. 18

Applying Eq. 18 to the results from process wafer 1, the model lines up well with the observed results at lower displacement values (Figure 88). This is consistent with the model assumption that the deflection is less that one half the diaphragm thickness. 


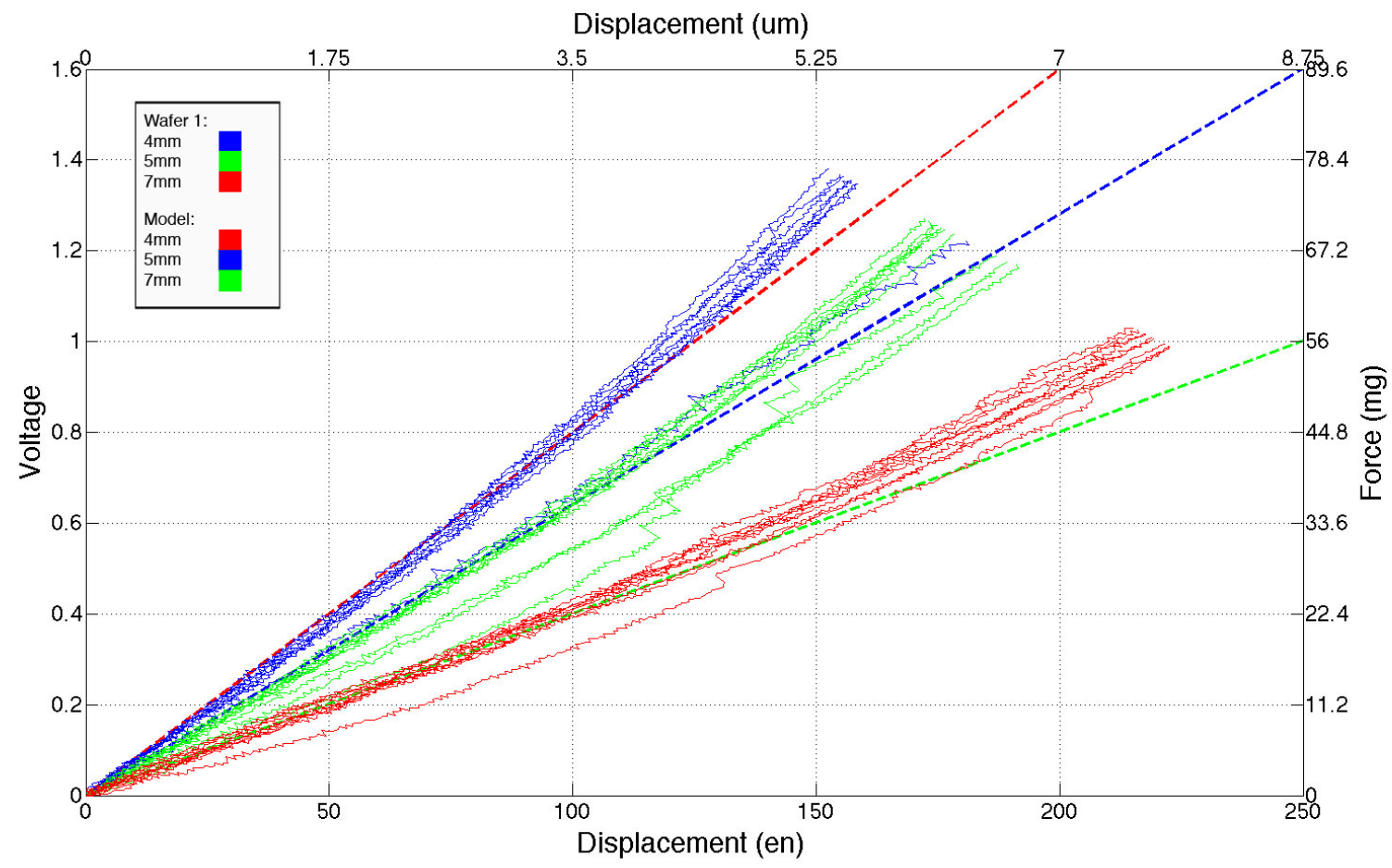

Figure 88: Process wafer 1 gage R\&R results as compared to theoretical model with correction formula.

Eq. 18 was then applied to process wafer 2, showing a systemic difference in the displacement (Figure 89). 


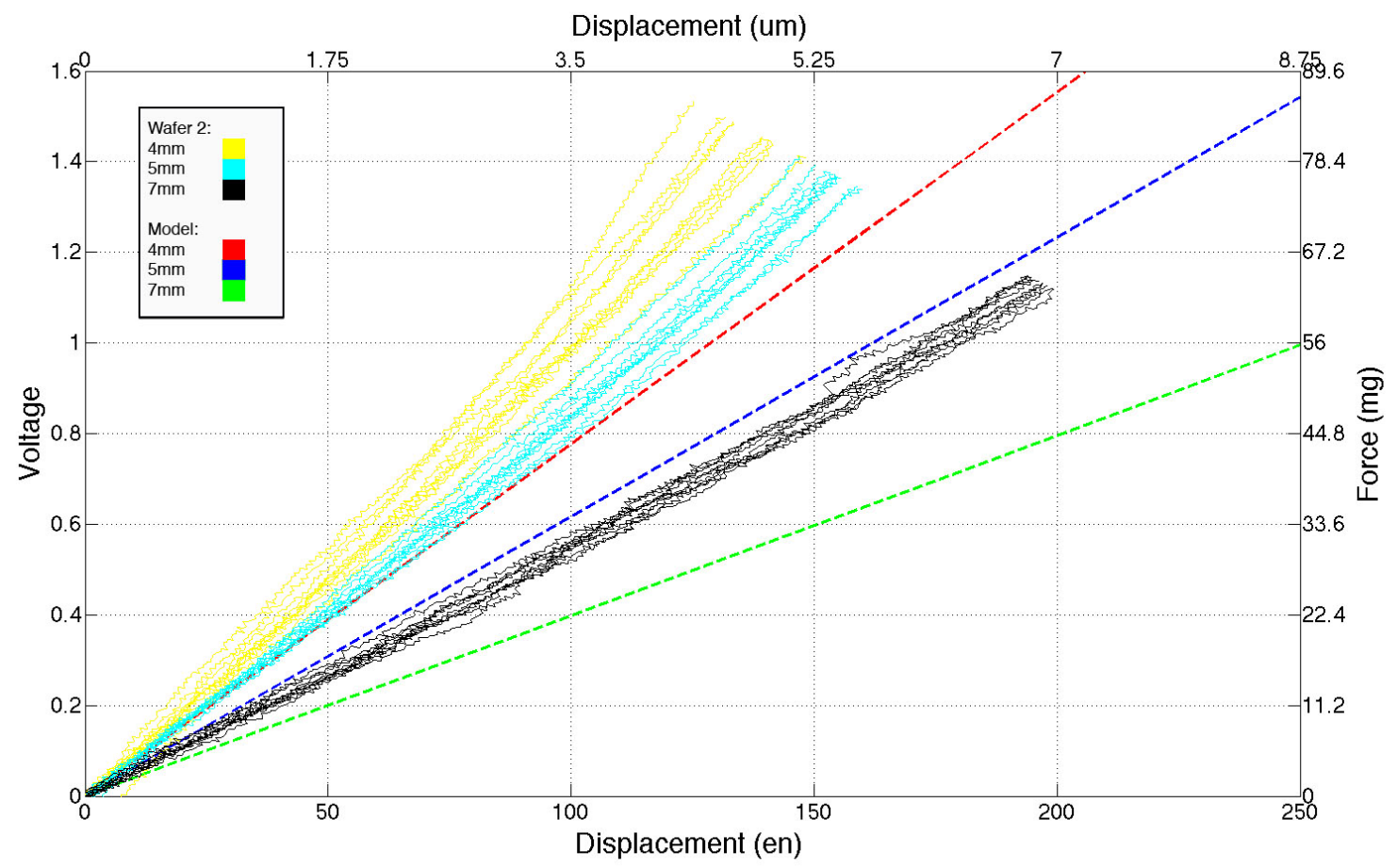

Figure 89: Process wafer 2 gage R\&R results as compared to theoretical model with correction formula.

The systemic displacement difference between process wafer 1 and 2 was compensated for through an adjustment of the $\mathrm{K}_{1}$ value of the model. This produced an increase in the slope of the line of approximately 25\% (Figure 90). 


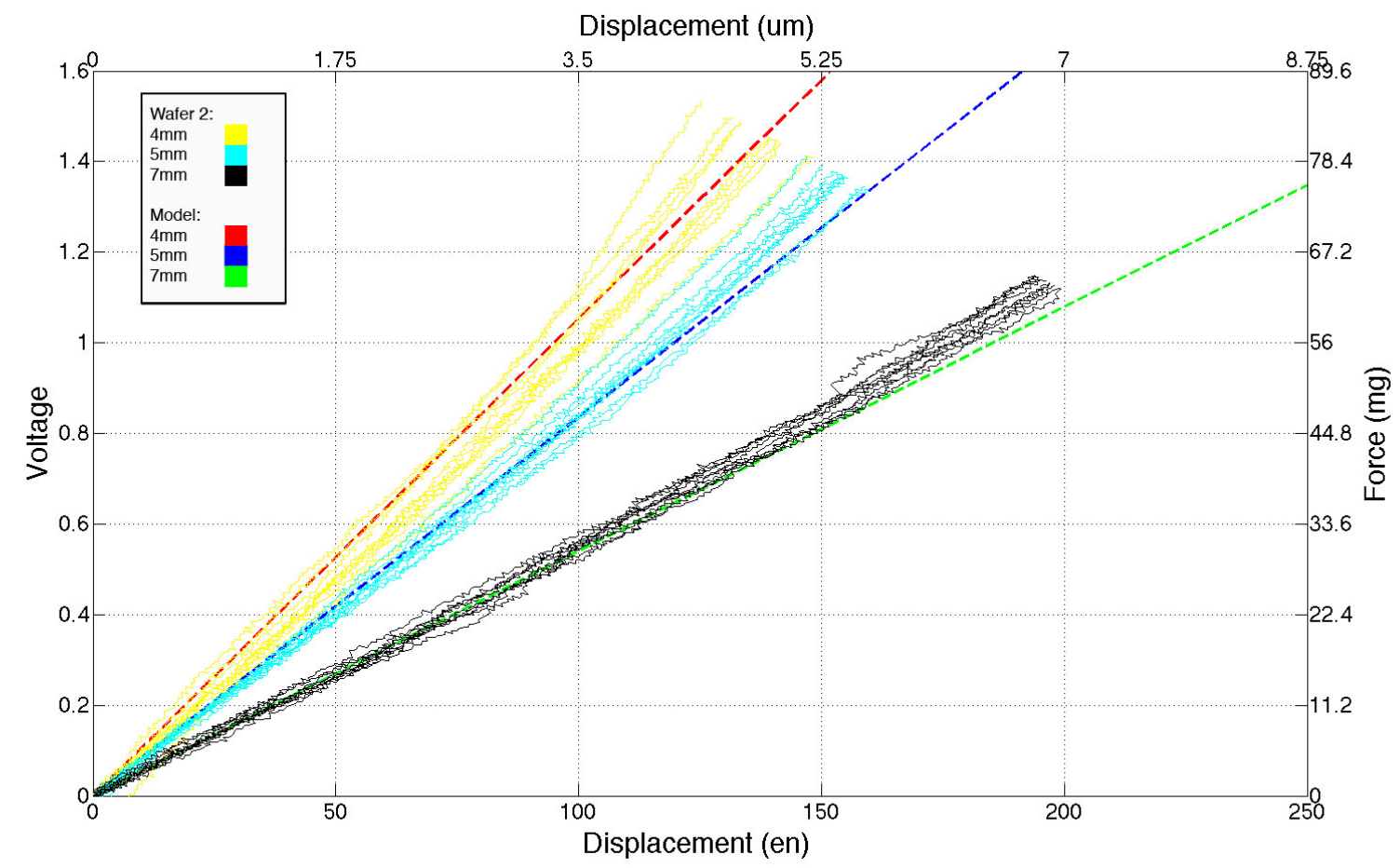

Figure 90: Process wafer 2 gage R\&R results as compared to theoretical model with correction formula and linear shift.

Through the model and compensation equation, a final model was developed to express the deflection of diaphragms based on their side length (b) and thickness (t) (in meters) (Eq. 19).

$$
y_{m}=k_{1} \frac{P b^{2}}{E t^{3}} \cdot \frac{1}{-19,000 b^{2}+340 b-310}
$$

Eq. 19

Eq. 19 can be subsequently used to estimate the force and displacement characteristics of diaphragms produced in the Microfabrication lab. 


\section{Discussion}

\subsection{MICRO FORCE DISPLACEMENT SYSTEM}

\subsubsection{HARDWARE}

The tip of the transducer was aligned using a bubble level to be normal to the surface being tested. Other methods of angular alignment could have been used to ensure a more orthogonal application of force. If the tip had not been orthogonal, the displacements produced would be less than the actual displacement of the transducer cantilever. To increase the maximum stiffness resolvable by the MFDS, the transducer cantilever could be stiffened with solder or epoxy, increasing the effective spring constant. The system would have to be recalibrated after such a change, and would have the disadvantage of loosing resolution on the low end of resolvable stiffness. Several different transducer tip radii could be used to ensure no stress concentrations are produced around the transducer tip during testing. Smaller transducer tips tend to create stresses due to the higher pressures produced. A vibration isolation table would reduce the noise observed in both the systemic displacement calibration as well as the transfer function calibration (currently the noise is approximately $\pm 0.25 \mathrm{mg}$ ).

\subsubsection{SOFTWARE}

The software of the system could be improved by changing the output units to microns to limit future confusion for new users. In addition, some of the post processing done in Matlab could be moved into the C\# code to provide a more complete output. The

crosshairs of the camera view could be expanded to the edges of the window to aid in the alignment of samples for testing. 


\subsubsection{CALIBRATION}

The accuracy of the $X \& Y$ axis calibration was sufficient for the testing of $4 \mathrm{~mm}$, $5 \mathrm{~mm}$, and $7 \mathrm{~mm}$ diaphragms, however for smaller diaphragms a more accurate calibration may be necessary. This calibration could be improved through the use of a gold sputtered microscope slide. Instead of silicone grease, the gold sputtered microscope slide would be contacted with the transducer probe, leaving a scratch, which could then be used as the reference for $X \& Y$ axis calibration. The specified $Z$ axis displacement was found to be accurate according to a microscope reticle, but could be further characterized with the help of a position sensitive device to expand upon the resolution and step size of the $Z$ axis.

The systemic displacement results could be improved through the collection of additional samples, which would increase the normality of the data. In addition, more samples would decrease the standard deviation, creating a more confident systemic displacement value.

The transfer function was hindered by the lack of appropriate analytical balance. A higher resolution balance is necessary to continue calibration of the MFDS in the current configuration. An alternative approach would be to use a calibrated cantilever or other spring to determine the force exerted by the transducer. An example of such a device is a nanoindenter AFM tip. Testing several different tips with different spring constants would allow for a more accurate measure of the transfer function. 


\subsection{DIAPHRAGMS}

\subsubsection{DESIGN}

The design of the test structures provided test structures that were measurable with the MFDS, and have the potential for future use as functionalized MEMS microphones. Although all diaphragms survived processing, larger diaphragms could have been made to test the practical limits of wet processing high aspect ratio silicon diaphragms.

\subsubsection{FABRICATION}

The largest problem encountered during the fabrication of the diaphragms was the pin holes created by the dual doped TMAH etch. These pin holes could have been created by either the buried oxide, or thermal oxide, however optical microscopy results indicated that the pin holes were larger on the buried oxide side of the diaphragms, suggesting that the buried oxide was the source of the defects. The SOI process introduces the defects in production. The yield of perfect diaphragms $(61 \%$ for wafer 1 , $50 \%$ for wafer 2 ) seems low, but when comparing the pin hole area to diaphragm area for a $4 \mathrm{~mm}$ diaphragm, the pin hole area is only $5.6 \mathrm{E}-3 \%$ of the total area. With such a small area of defect, the mechanical properties should not be affected.

The thickness of the diaphragms was not found to be different than the nominal thickness of the device layer of the SOI wafers. Difficulty in sputtering gold on the edges contributed to charging and large measurement tolerances in the thickness of the diaphragms. Theoretically the diaphragms should be thinner than the 10 um specification due to consumption of silicon during the thermal oxide process, but higher quality SEM imaging is necessary to resolve the change in thickness.

For such thin diaphragms, Fourier Transform Infra-Red Spectrometry (FTIR) could be utilized to scan each diaphragm and analyze the thickness. The SEM cross 
section method only gave results at one cross section of the diaphragm, however FTIR would be able to resolve the thickness of the diaphragms at all points in a non destructive manner.

In the future, a thick thermal oxide could be grown to change the thickness of the device layer prior to growing the thermal oxide mask. Using a thermal oxide to remove some of the silicon device layer would allow for fine adjustment of the diaphragm thickness, however non-uniformity in thermal oxides could lead to different diaphragm thicknesses depending on the wafer position within the furnace.

\subsubsection{GAGE R\&R STUDY}

The results from process wafer 1 and 2 show a shift between the stiffness of each wafer. In addition, the variation was larger for wafer 1 than wafer 2 . It is possible that the mounting of the entire wafer for process wafer 1 lead to more inconsistent results. The data from the Gage R\&R test was analyzed to see if diaphragms from wafer 1 that were located in the middle of the wafer tested different than diaphragms towards the edge of the wafer, but no statistical difference was found. This suggests that if the mounting was affecting the stiffness of the diaphragms, then the results should not differ based on the location of the diaphragm. Another potential source of difference in stiffness was the difference in thickness of the handle layers. There was a small difference in the thickness of the thermal oxide grown between process wafer 1 and process wafer 2, which could have an effect on the results. The difference between wafers was only $114 \AA$, which would only change the silicon device layer thickness by $52 \AA$, or $0.052 \%$, which would not have as large of an effect on the stiffness as was observed. If the buried oxide thickness was significantly different between the two wafers, there could be more effect on the stiffness of the diaphragms. 


\subsubsection{COMPARISON OF TEST RESULTS TO MODELING}

Both process wafers tested to have systemic differences in stiffness that were statistically significant. The linear model used to model the diaphragms prior to fabrication was also found to be different from the two process wafers, however the theoretical results are more spread out than the observed results. The probe tip of the transducer had a radius of approximately 5um, which is approaching a point force in terms of force application. Having such a small area to apply force over can cause extra stress around the application area, reducing the displacement of the diaphragms. The edge conditions chosen for modeling were clamped edges. In reality the edges of the diaphragms were clamped on the bottom, but free on the top of the diaphragms, potentially leading to slightly more displacement than predicted in the model. In addition, the Young's Modulus and poisons ratio could differ from the actual material properties of the silicon in the diaphragms. The compensated linear model takes into account all of the material variables, allowing future students to predict the behavior of diaphragms created from similar SOI wafers in the Microfabrication Lab. An attempt was made to model the diaphragms in SolidWorks Simulator, however the aspect ratio of the diaphragms made the mesh size too small to run the model in a reasonable amount of time on the computers avalible. 


\section{CONCLUSIONS}

\subsection{MICRO FORCE DISPLACEMENT SYSTEM}

The MFDS was created for $\$ 3378$ in new parts. The hardware of the system was chosen with an emphasis on repairability and improvement by future students. The software of the system was created with an easy to use GUI as well as versatile open source software allowing for future customization. The MFDS achieved displacement resolution of approximately $35 \mathrm{~nm}$, in the same order of magnitude as the other two systems being used for measurements of this scale. The MFDS has the capability to test samples of stiffness under $279 \mathrm{mg} / \mathrm{um}$, with an equipment variability of $0.0185 \mathrm{~V}$ $(1.02 \mathrm{mg})$ at $1.75 \mathrm{um}$ and $0.0337 \mathrm{~V}(1.86 \mathrm{mg})$ at $3.5 \mathrm{um}$. The MFDS was capable of resolving differences in silicon diaphragm of sizes: $4 \mathrm{~mm}, 5 \mathrm{~mm}$, and $7 \mathrm{~mm}$ and $10 \mathrm{um}$ thickness.

\subsection{DIAPHRAGMS}

Silicon diaphragms were created through a dual doped TMAH etch, with side lengths less than $2.75 \%$ different than the nominal side length. The dual doped TMAH solution was shown to exhibit minimal hillock formation, and produce sidewall angles consistent with literature values. The diaphragms created were robust enough to survive the wet etching process without failure, making the devices suitable for future functionalized MEMS devices. The process wafers had a yield of over $50 \%$ of perfect diaphragms, and $96 \%$ for diaphragms suitable for mechanical testing. The diaphragms were found to have warpage of less than $250 \mathrm{~nm}$ for $7 \mathrm{~mm}$ diaphragms. 


\section{RECOMMENDATIONS FOR FUTURE WORK}

The largest limiting factor in the current design of the MFDS is the lack of an accurate transfer function. Through the use of a more accurate analytical balance (such as a model with a resolution of $0.01 \mathrm{mg}$ ), confidence bounds can be attached to the transfer function.

A vibration isolation table would eliminate noise from other equipment present in the Microfabrication Lab. The location of the MFDS on a stainless steel table transfers noise from surrounding equipment as well as people moving near the MFDS. A vibration isolation table would allow the use of the MFDS during high traffic times of the day without data errors from vibrational disturbances. A vibration isolation table would help to decrease noise in both the systemic displacement results as well as future transfer function results.

The addition of a camera ring light would make selecting the test site on the testing specimen faster and more accurate. If additional extension tubes were used on the camera to increase the magnification of the lens, the ring light would be necessary to provide enough light to image the testing specimens.

Some software improvement could be accomplished to provide additional functionality to the user. A micrometer on the camera view of the software would allow the user to quantify the size of the testing specimens. Updating the live force displacement graph by accounting for the systemic displacement would provide a more accurate picture of the testing conditions to the user. With significant modifications in the software, automated tests could be performed to test all the devices on a wafer. In addition, the MFDS could be used as either a profilometer or AFM style 3D scanner by 
converting the transducer output voltage into a corresponding displacement using the systemic displacement equation.

The test diaphragms have the potential to be used for a variety of applications. With the addition of a piezoelectric layer, the diaphragms could be actuated as speakers or used as pressure sensors. The diaphragms could also be actuated through an electrostatic approach, using a sputtered metal layer and a thin gap to apply a voltage across creating a force.

Most diaphragms used in MEMS applications are actuated by, or create a pressure, not a point force. The MFDS is not currently set up to apply pressures over the entire area of the diaphragm, which could be improved upon to make the test set up more closely mimic real world applications. However for cantilever characterization, the MFDS is appropriate. 


\section{REFERENCES}

1. Microelectromechanical Systems (National Research Council, 1997)

2. S. Meredith, M.S. thesis, California Polytechnic State University- San Luis Obispo, 2009.

3. R. E. Hummel, Electronic Properties of Materials (Springer Science+Business Media, New York, 2001), pp. 149-152.

4. J. W. Judy, Smart Materials and Structures 10, 1115-1134 (2001).

5. S. A. Campbell, Fabrication Engineering at the Micro and Nanoscale (Oxford University Press, New York, 2008), pp. 555-593.

6. Brooks, E.L., The Design and Fabrication of an Electrostatically Actuated Diaphragm with a Silicon-On-Insulator Wafer, in Materials Engineering. 2013, California Polytechnic University: San Luis Obispo.

7. MEMS and Nanotechnology Applications. 2014; Available from: https://www.mems-exchange.org/MEMS/applications.html.

8. SMI. MEMS Pressure Sensing Solutions. 2013 2/2/2014 8]; Available from: http://www.simicro.com/ci2/userfiles/files/SMI\%20Automotive\%20Brochure\%20final.pdf.

9. MEMS Microphones Broadcast Strong Growth, in Solid State Technology. 2014, Extension Media.

10. Dixon-Warren, S.J., Overview of MEMS Microphone Technologies for Consumer Applications. MEMS Investor Journal, 2011.

11. Multitest, Strategic Approaches for MEMS Test (multitest.com) pp.1-18.

12. Waters, P., Stress Analysis and Mechanical Characterization of Thin Films for Microelectronics and MEMS Applications, in Mechanical Engineering. 2008, University of South Florida. 
13. "Model 5948 MicroTester for Small-Scale Low-Force Testing up to 2 kN," http://www.instron.us/wa/product/MicroTester-System-for-Low-Force-Static.aspx (10 May 2013).

14. MIT. Material Property Database: SU-8 Photoresist. 2014 [cited 2014; Available from: http://www.mit.edu/ 6.777/matprops/su-8.htm.

15. K. Biswas, S.K., Etch Characteristics of $\mathrm{KOH}, \mathrm{TMAH}$, and dual doped TMAH for Bulk Micromachining of Silicon. Microelectronics Journal, 2005. 37: p. 519-525.

16. Carvill, J., Mechanical Engineer's Data Handbook. 2003, Burlington, MA: Elsevier Science.

17. T. Hsu, MEMS \& Microsystems Design and Manufacture (McGraw-Hill, New York, 2002), pp. 96-162.

18. Ando, T.; Xueping Li; Nakao, S.; Kasai, T.; Shikida, M.; Sato, K., "Effect of crystal orientation on fracture strength and fracture toughness of single crystal silicon," Micro Electro Mechanical Systems, 2004. 17th IEEE International Conference on. (MEMS) , vol., no., pp.177,180, 2004doi: 10.1109/MEMS.2004.1290551

19. Grove, B.E.D.A.S., General Relationship for the Thermal Oxidation of Silicon. Journal of Applied Physics, 1965. 36(12): p. 3770-3778.

20. Cheung, N. (n.d.). Thermal oxidation of si. Informally published manuscript, U.C. Berkeley Electrical Engineering, Retrieved from http://www.eng.tau.ac.il/ yosish/courses/vlsi1//-4-1-Oxidation.pdf

21. P. Sullivan, B.W.O., M. E. Aklufi, Tetra-Methyl Ammonium Hydroxide (TMAH) Preferential Etching for Infared Pixel Arrays. 2000: SSC San Diego.

22. A Pandy, L.M.L., B. Nikpour, M. Paranjape, M. Kahrizi, Experimental Investigation of High Si/Al Selectivity During Anisotropic Etching in Tetra-Methyl Ammonium Hydroxide. Journal of Vacuum Science \& Technology A, 1998. 16(2): 

p. $868-872$.

23. Duan, X., Microfabrication Using Bulk Wet Etching with TMAH, in Department of Physics. McGill University: Montreal, Quebec.

24. Measurement Systems Analysis: Reference Manual. 3 ed. 2002: DaimlerChrysler Corporation, Ford Motor Company, General Motors Corporation.

25. G. B. Hughes (personal communication, October, 2013-March 2014). 


\section{APPENDIX I}

using System;

using System.Collections.Generic;

using System.Linq;

using System. Text;

using System.Threading.Tasks;

using System.IO.Ports;

using System.IO;

namespace MFDMInterface

\{

class StageSerialCom

\{

const int BAUD = 19200;

SerialPort StagePort;

public StageSerialCom(string comPort)

\{

StagePort $=$ new SerialPort (comPort);

StagePort. BaudRate = BAUD;

StagePort. Parity = Parity. None;

StagePort. DataBits $=8$;

StagePort.StopBits = StopBits.One;

StagePort. Handshake $=$ Handshake. XOnXOff;

StagePort.Open();

SetUnits $(1,0)$;

SetUnits $(2,0)$;

SetResolution(1, 1);

SetResolution (2, 1);

SetMaxAccDec (1, 50000);

$\operatorname{SetMaxAccDec}(2,50000)$;

SetAcceleration (1, 50000);

SetAcceleration (2, 50000);

SetDeceleration (1, 50000);

SetDeceleration(2, 50000);

SetMaxVelocity(1, 141374);

SetMaxVelocity(2, 141374);

SetVelocity (1, 70000);

\}

SetVelocity (2, 70000);

public void SendCommand(int stageNum, string command, int setting)

StagePort.Write(stageNum + command + setting + "\r");

public void SetMaxVelocity(int stageNum, int maxVelocity)

SendCommand(stageNum, "VU", maxVelocity);

public void SetVelocity(int stageNum, int velocity)

SendCommand(stageNum, "VA", velocity);

public void SetResolution(int stageNum, int resolution)

\{

SendCommand(stageNum, "SU", resolution); 


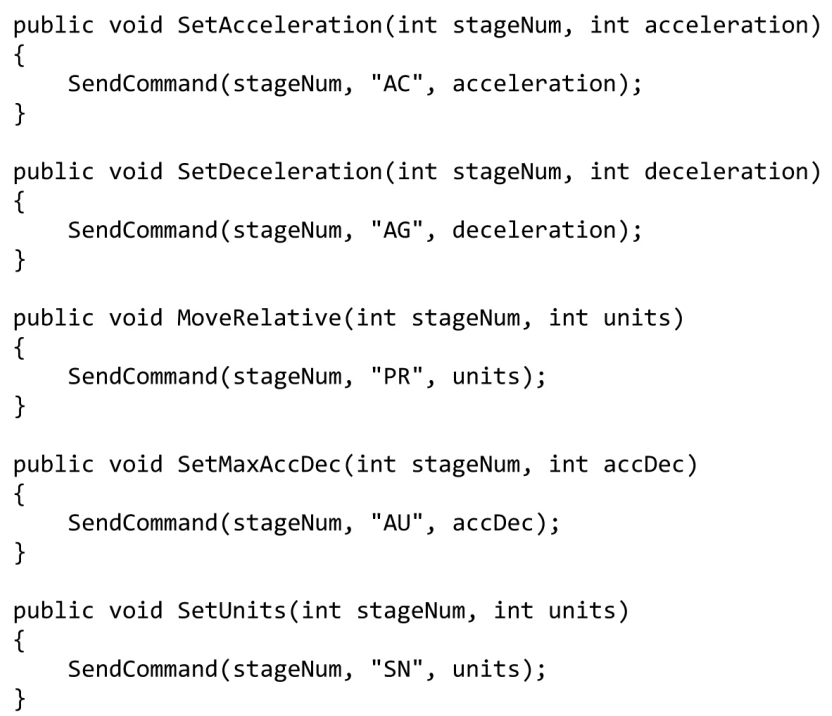


using System;

using System. Threading;

using System.Collections.Generic;

using System.IO.Ports;

using System.Linq;

using System. Text;

using System. Threading.Tasks;

namespace MFDMInterface

\{

class Testingutility

private static StageController MovementController;

private static SerialPort KeithleyPort;

public static Boolean KeepRunningTest;

public delegate void DataUpdateDelegate(float displacement, float voltage);

private class TestRunner

private DataUpdateDelegate GraphUpdate;

private float StopVoltage;

private int ReadingDelay;

private int StepSize;

private string OutFile;

public TestRunner(DataUpdateDelegate graphUpdate, float stopVoltage, int readingDelay, int stepsize, string outFile)

GraphUpdate = graphUpdate

StopVoltage $=$ stopVoltage

ReadingDelay = readingDelay;

StepSize = stepSize;

\}

OutFile = outFile

public void RunTest()

\{

float curvoltage;

float displacement $=0$;

string strVolt;

OutFile);

System.IO.StreamWriter file = new System.IO.StreamWriter(@"C: \Users\microfab\Desktop \" +

file.Write("Date: " + DateTime.Now + " Stop Voltage:
$"$ + ReadingDelay + " (ms) Step Size: " + StepSize + " (en) \n");

do

\{

MovementController.ZNegative(StepSize);

displacement $+=$ StepSize;

System.Threading. Thread.Sleep(ReadingDelay);

KeithleyPort.Write(":READ? \r");

strVolt = KeithleyPort.ReadLine ();

file.Write (displacement + "," + strVolt);

curVoltage = float.Parse(strVolt, System.Globalization.CultureInfo.InvariantCulture); GraphUpdate (displacement, curVoltage);

\} while (curVoltage $>=$ StopVoltage \&\& KeepRunningTest);

file.close ();

\}

KeithleyPort.Close();

public TestingUtility(StageController stageCont, string keithleyPort)

\{ 


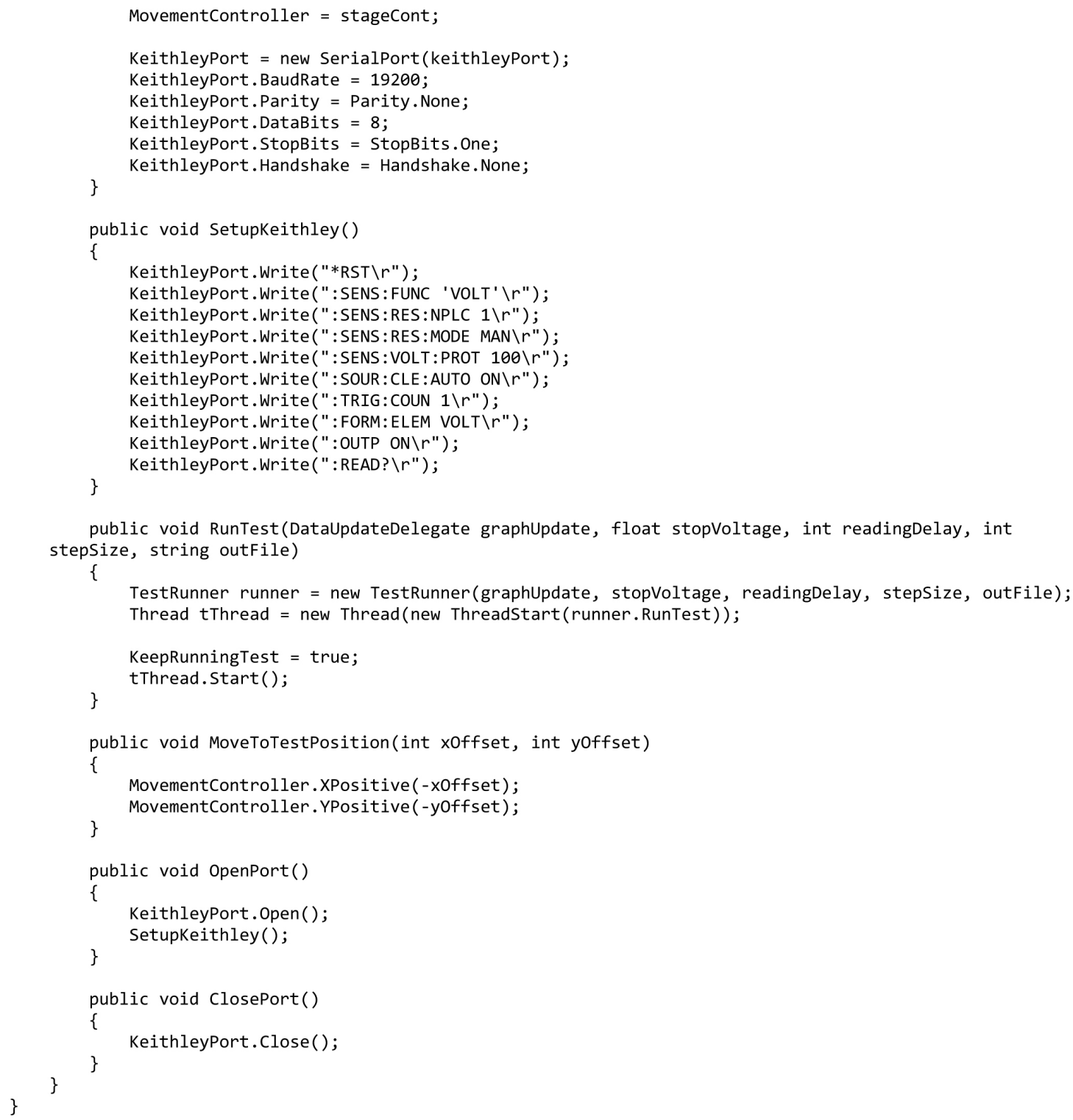

public void RunTest(DataUpdateDelegate graphUpdate, float stopVoltage, int readingDelay, int stepSize, string outFile)

\{ TestRunner runner $=$ new TestRunner (graphUpdate, stopVoltage, readingDelay, stepSize, outFile); Thread tThread $=$ new Thread(new ThreadStart (runner. RunTest)); 


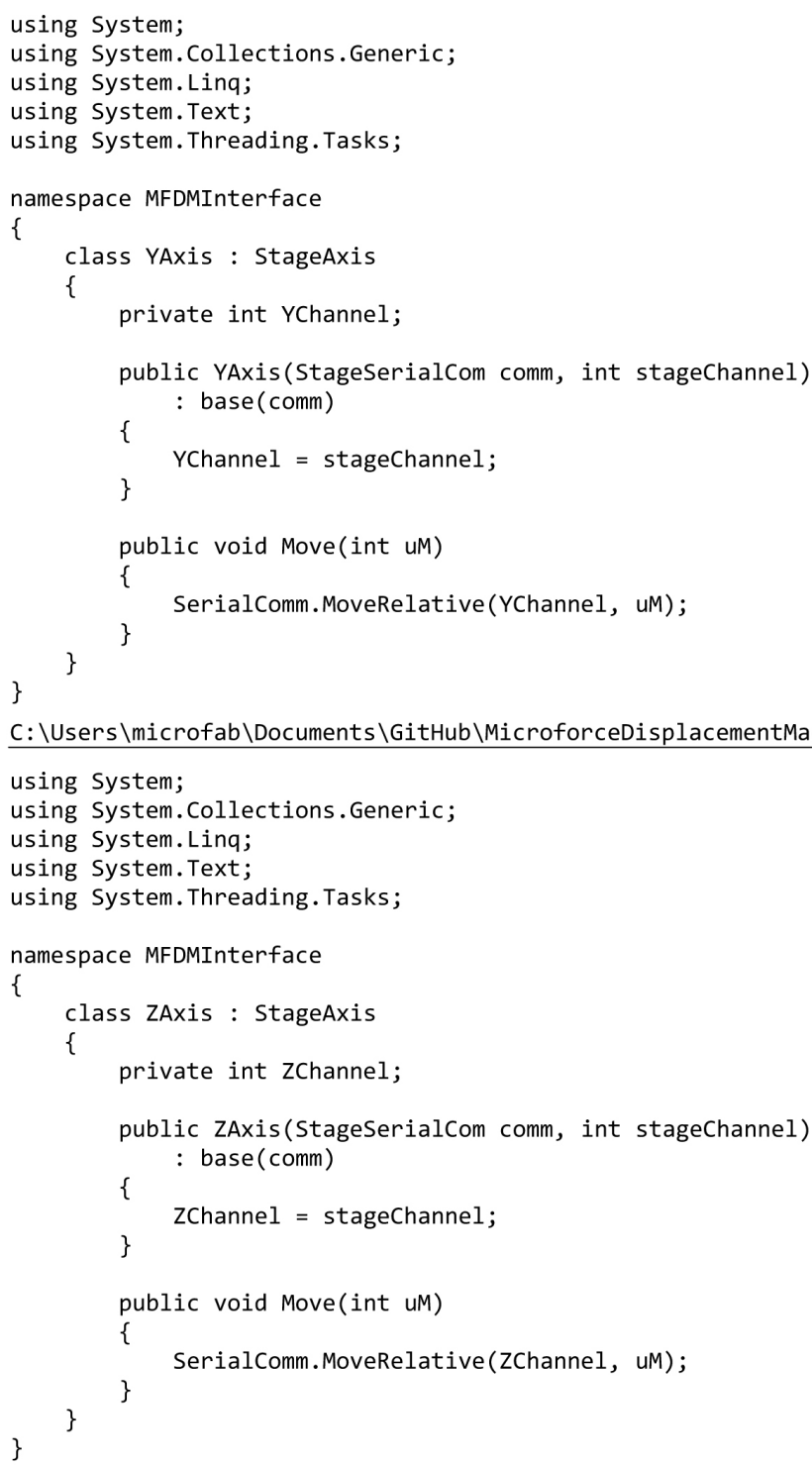




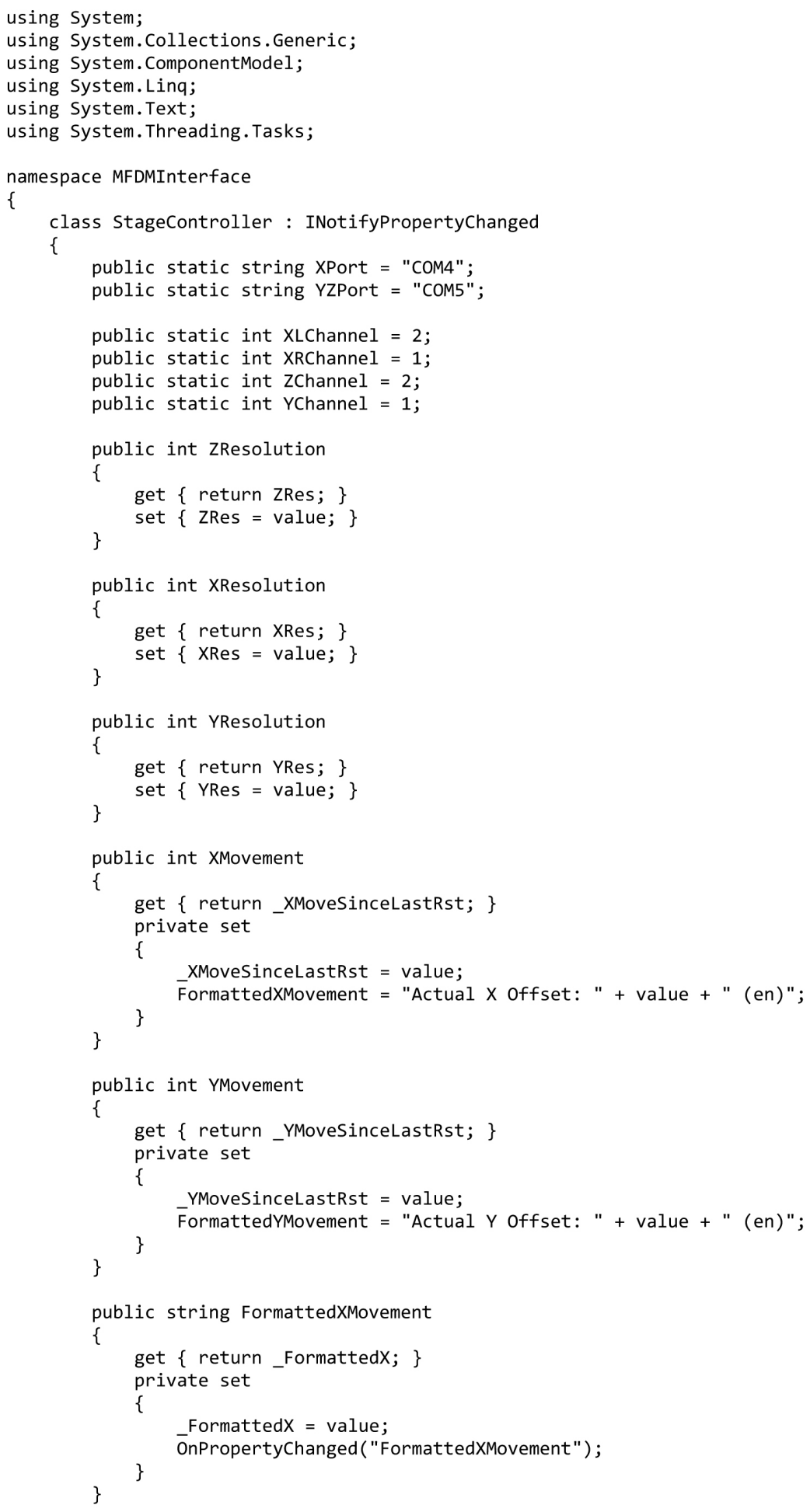




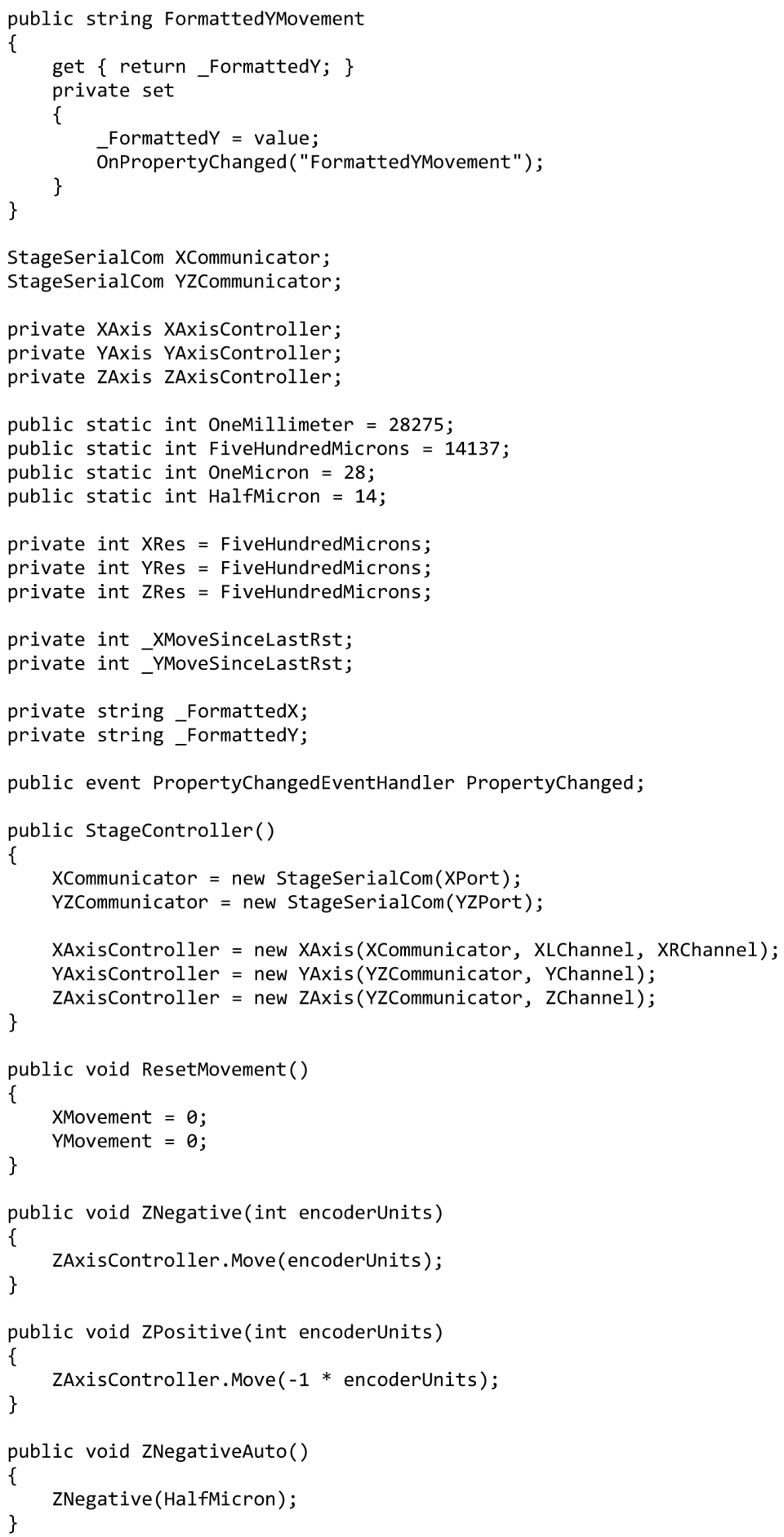




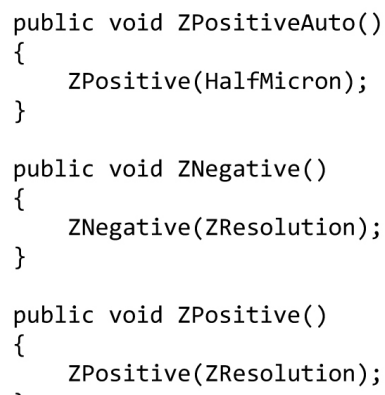


using System;

using System.Collections.Generic;

using System.ComponentModel;

using System.Data;

using System.Drawing;

using System.Linq;

using System. Text;

using System. Threading.Tasks;

using System.Windows. Forms;

using System.Windows.Forms.DataVisualization.Charting;

namespace MFDMInterface

public partial class Form1 : Form

private StageController MovementController;

private CalibrationUtility CalUtill;

private TestingUtility Testutil;

delegate void UpdateGraphCallback(float displacement, float voltage);

public Form1()

InitializeComponent();

MovementController $=$ new StageController () ;

Calutill = new CalibrationUtility(MovementController, "COM6", "COM3"); //THIS IS THE LINE (Move $\boldsymbol{k}$ ...roller, Balance, Keithley)

\} Testutil = new TestingUtility (MovementController, "COM3");

private void Form1_Load(object sender, EventArgs e)

assXOffLabel. Text = "Assumed X Offset: " + CalUtill.XOffset + "(en)";

assYOffLabel. Text = "Assumed Y Offset: " + CalUtill. YOffset + " (en)";

forcelabel. Text = "Force: - $(\mathrm{mg}) "$;

voltageLabel. Text = "Voltage: -- (v)";

actXOffLabel.DataBindings.Add(new Binding("Text", MovementController, "FormattedXMovement")); actYOffLabel.DataBindings.Add(new Binding("Text", MovementController, "FormattedYMovement")); MovementController.ResetMovement();

verticalStepLabel.Text = "Vertical Step Size: " + verticalStepBar.Value + " (en)";

stepSizeLabel.Text = "Horizontal Step Size: " + XYStepBar.Value + "(en)";

forceDisplacementChart.Series[0].ChartType = System.Windows. Forms.DataVisualization. Charting. SeriesChartType.Line;

forceDisplacementChart.Series[0].Name = "Force vs. Displacement";

forceDisplacementChart.ChartAreas[0].AxisX.Title = "Displacement (en)";

forceDisplacementChart.ChartAreas[0].AxisY.Title = "Force (mg)";

if (!icImagingControl1.DeviceValid)

icImagingControl1. ShowDeviceSettingsDialog( );

if (! icImagingControl1.DeviceValid)

\{

MessageBox.Show("No device was selected.", "Grabbing an Image", \} this.Close( );

int width = icImagingControl1. Width;

int height $=$ icImagingControl1. Height;

icImagingControl1. LiveDisplayDefault = false

icImagingControl1. LiveDisplayHeight = icImagingControl1. Height;

icImagingControl1. LiveDisplayWidth = icImagingControl1. Width; 


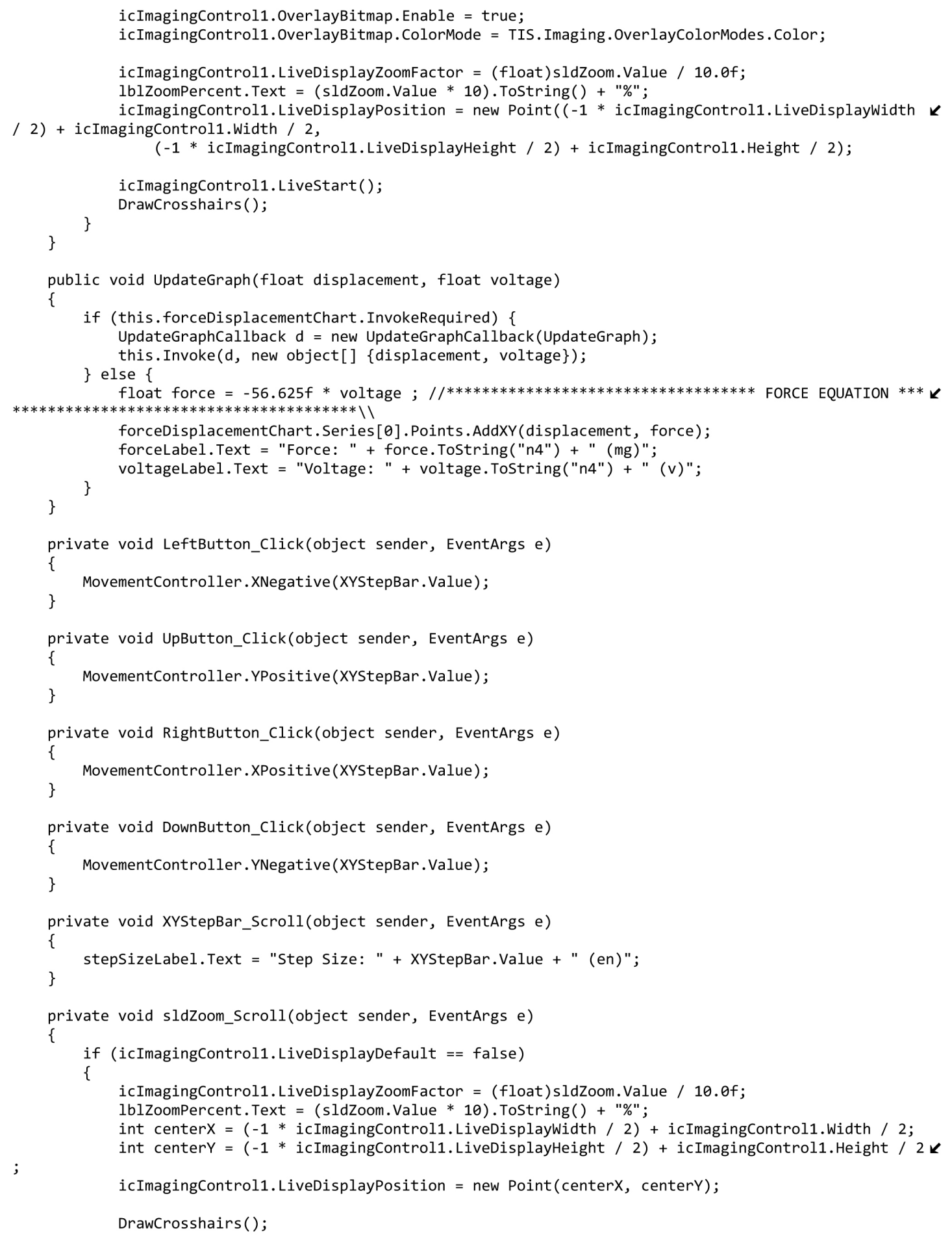


\{

False!");

MessageBox.Show("The zoom factor can only be set" + "\n" + "if LiveDisplayDefault returns

\}

private void DrawCrosshairs()

\{

int width = icImagingControl1. Imagewidth;

int height = icImagingControl1. ImageHeight;

int crosshairLength = (icImagingControl1. Imagewidth / icImagingControll.LiveDisplayWidth) * 50;

icImagingControl1.OverlayBitmap.Fill(icImagingControl1.OverlayBitmap.DropOutColor);

for (int $i=\operatorname{Math} . \operatorname{Max}(0,7-\operatorname{sldZoom.Value)} ; i>=0 ; i--$ )

\{

icImagingControl1. OverlayBitmap.DrawLine(Color. Red, width / 2 - crosshairLength, height / 2 K , width / 2 + crosshairLength, height / 2);

icImagingControl1. OverlayBitmap.DrawLine(Color.Red, width / 2 , height / 2 - crosshairLength $\boldsymbol{k}$

, width / 2, height / 2 + crosshairLength);

icImagingControl1.OverlayBitmap.DrawLine(Color.Red, width / 2 - crosshairLength, height / 2 K

$+i$, width $/ 2+$ crosshairLength, height / $2+i)$;

icImagingControl1. OverlayBitmap.Drawline(Color.Red, width / $2+i$, height / 2 -

crosshairLength, width / $2+i$, height / $2+$ crosshairLength);

icImagingControl1. OverlayBitmap. DrawLine(Color. Red, width / 2 - crosshairLength, height / 2 K

- i, width / 2 + crosshairLength, height / 2 - i);

icImagingControl1. OverlayBitmap. DrawLine(Color.Red, width / 2 - i, height / 2 -

crosshairLength, width / 2 - i, height / 2 + crosshairLength);

\}

private void probeUp_Click(object sender, EventArgs e)

\{

MovementController.ZPositive(verticalstepBar.Value);

private void probeDown_Click(object sender, EventArgs e)

MovementController.ZNegative(verticalstepBar.Value);

private void bcCal_Click(object sender, EventArgs e)

\{

CalUtill.OpenPorts();

forceDisplacementChart.Series [0]. Points.Clear();

CalUtill.GenerateBalanceKeithleyCalibrationData(new CalibrationUtility.DataUpdateDelegate

(UpdateGraph), float.Parse(stopValue.Text), int.Parse(readingDelay.Text), int.Parse(stepSize.Text), outFile.Text);

\}

private void verticalstepBar_Scroll(object sender, EventArgs e) \}

verticalStepLabel.Text = "Vertical Step Size: " + verticalStepBar.Value + " (en)";

private void calButton_Click(object sender, EventArgs e)

\{

MovementController. ResetMovement();

MovementController.XPositive(CalUtill.Xoffset);

\}

MovementController.YPositive(Calutill.Yoffset);

private void setButton_Click(object sender, EventArgs e) 
\{

CalUtill. XOffset $=$ MovementController.XMovement;

CalUtill. YOffset $=$ MovementController. YMovement;

assXOffLabel. Text = "Assumed X Offset: " + CalUtill.XOffset + "(en)";

assYoffLabel. Text = "Assumed Y Offset: " + CalUtill. Yoffset + " (en)";

actXOffLabel. Text = "Actual X Offset: " + CalUtill.XOffset + " (en)";

\} actYoffLabel. Text = "Actual Y Offset: " + CalUtill.YOffset + " (en)";

private void backtrackButton_Click(object sender, EventArgs e)

MovementController.XPositive(-CalUtill.XOffset); MovementController.YPositive(-CalUtill. Yoffset);

private void runButton_Click(object sender, EventArgs e)

Testutil.OpenPort();

forceDisplacementChart.Series [0].Points.Clear( );

TestUtil.RunTest(new TestingUtility.DataUpdateDelegate(UpdateGraph), float.Parse

(stopVoltageText.Text), int.Parse(readingDelay.Text), int.Parse(stepSize.Text), outFile.Text);

\}

private void testPosition_Click(object sender, EventArgs e)

\{

TestUtil.MoveToTestPosition(CalUtill.XOffset, CalUtill.Yoffset);

private void stopButton_Click(object sender, EventArgs e)

Testingutility.KeepRunningTest = false;

CalibrationUtility. KeepRunningTest = false;

forceLabel. Text = "Force: - - (mg)";

\}

voltageLabel. Text = "Voltage: -- (v)"; 
using System;

using System.Collections.Generic;

using System.Linq;

using System. Text;

using System.Threading.Tasks;

using System.IO.Ports;

using System.IO;

using System. Threading;

namespace MFDMInterface

class CalibrationUtility

\{

private static StageController MovementController;

private static SerialPort BalancePort;

private static SerialPort KeithleyPort;

public static Boolean KeepRunningTest;

public int Xoffset;

public int Yoffset;

public delegate void DataUpdateDelegate(float displacement, float voltage);

private class CalibrationRunner

\{

private DataUpdateDelegate GraphUpdate;

private float StopValue;

private int ReadingDelay;

private int StepSize;

private string OutFile;

public CalibrationRunner(DataUpdateDelegate graphUpdate, float stopVoltage, int readingDelay, int stepSize, string outFile)

\{

GraphUpdate = graphUpdate;

StopValue = stopVoltage;

ReadingDelay = readingDelay;

StepSize = stepSize;

OutFile = outFile;

public void RunTest()

float curPressure;

char [] splitchars $=\left\{{ }^{\prime}\right.$ ' $\}$;

string result;

string[] splitResult;

float displacement $=0$;

string strVolt;

OutFile)

System.IO.StreamWriter file = new System.IO.StreamWriter(@"C: \Users \microfab\Desktop \" +

do

MovementController.ZNegative(StepSize);

displacement $+=$ StepSize;

System.Threading.Thread.Sleep(ReadingDelay);

BalancePort.Write("!KP $\backslash r$ ");

result $=$ BalancePort $\cdot$ ReadLine ();

splitResult $=$ result. Split $($ splitChars $)$;

foreach (string str in splitResult)

\{

if (str.Contains('.'))

\{ 


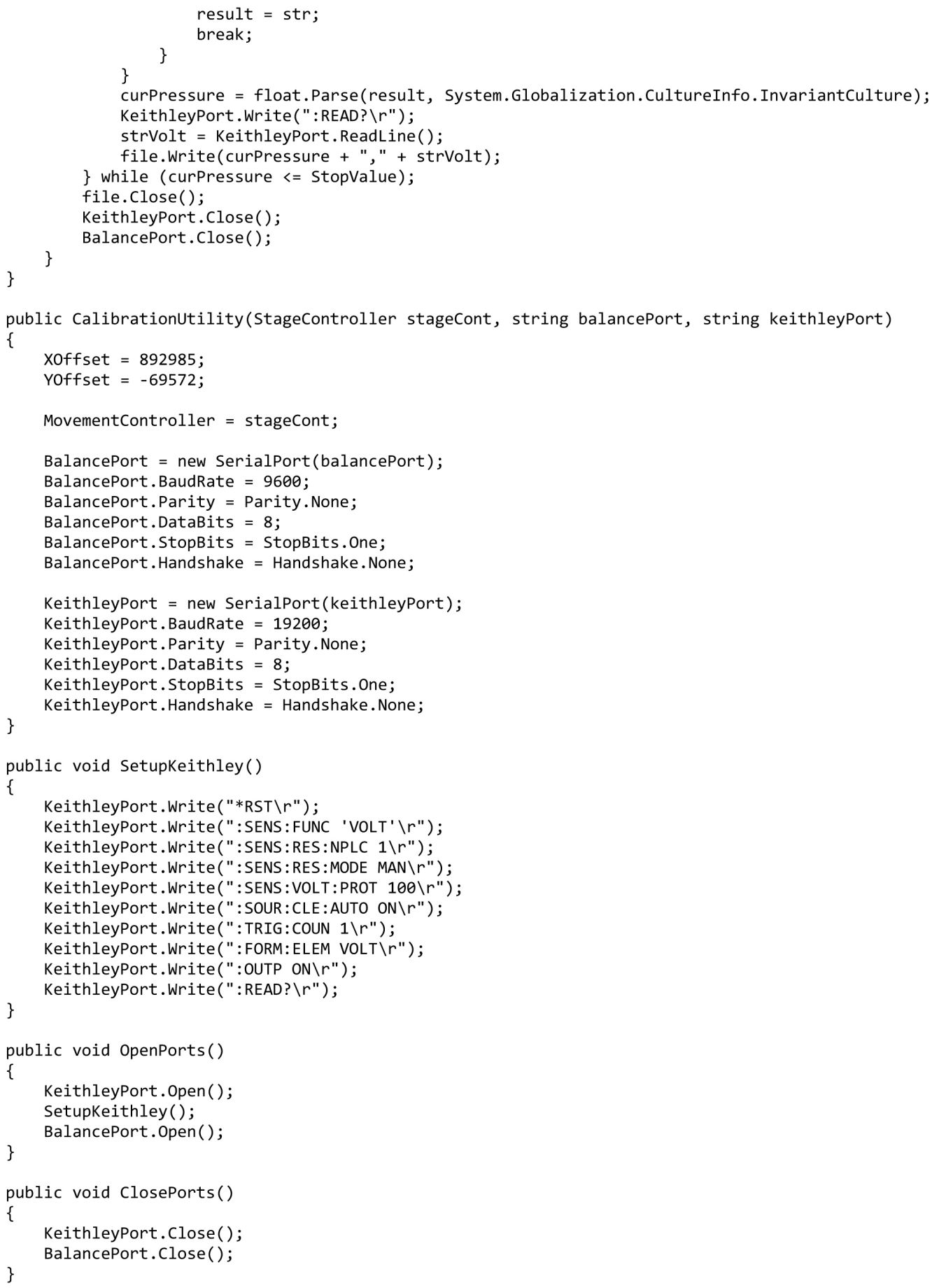
int readingDelay, int stepsize, string outFile) 
\{ CalibrationRunner runner = new CalibrationRunner (graphUpdate, stopValue, readingDelay, stepSize $\boldsymbol{K}$ , outFile);

Thread thread $=$ new Thread(new ThreadStart(runner. RunTest));

KeepRunningTest $=$ true;

\} tThread.Start ();

\}

\} 


\section{APPENDIX II}
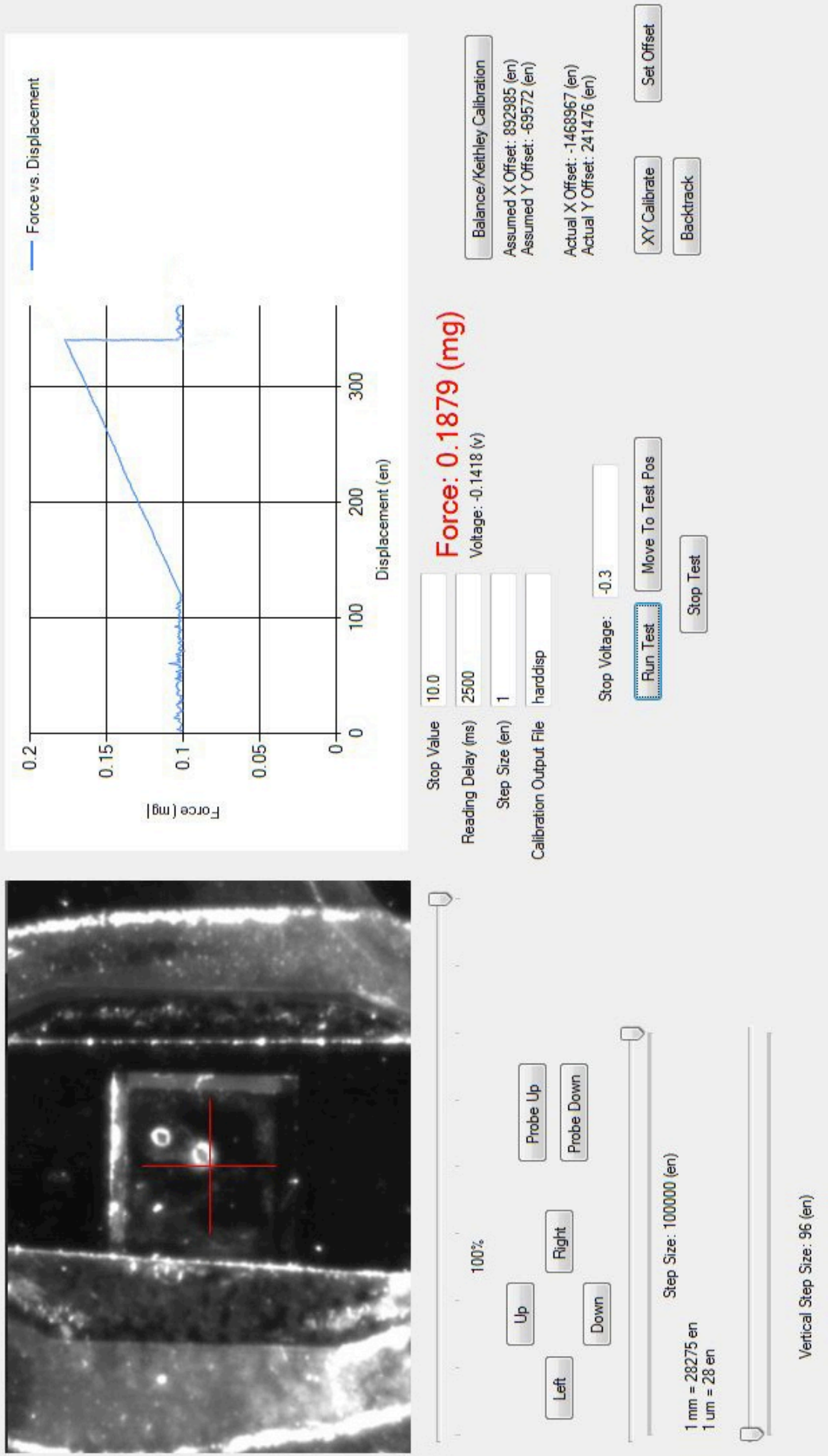


\section{APPENDIX III}

StANDARD OPERATING PROCEDURES FOR MICRO FORCE DISPLACEMENT MACHINE

$5 / 2014$

\section{Evan Cate}




\section{THEORY}

The system works by lowering the transducer (Figure 91) onto the sample being tested. The transducer is lowered in a quazi-static manner, with a delay in between each reading ('Reading Delay (ms)') and by specified step size ('Step Size (en)')(Figure 92).

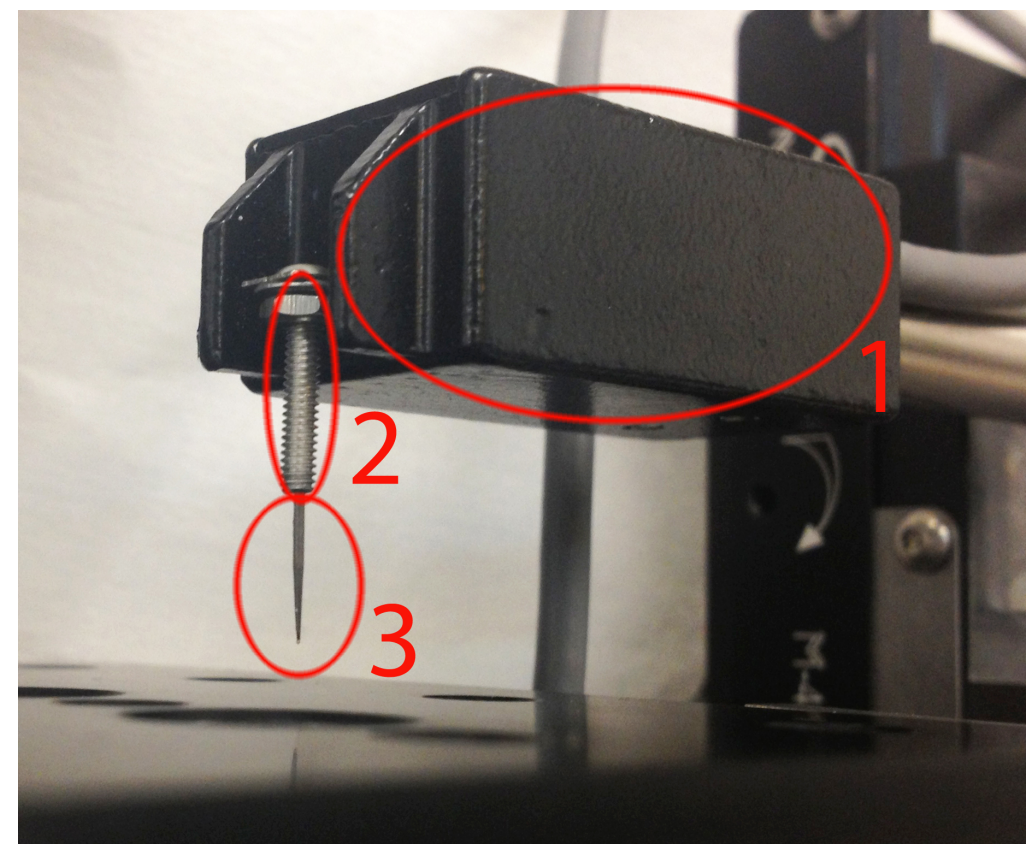

Figure 91: Transducer sub-assembly showing 1) transducer body, 2) vented screw, and 3) Microprober tip.

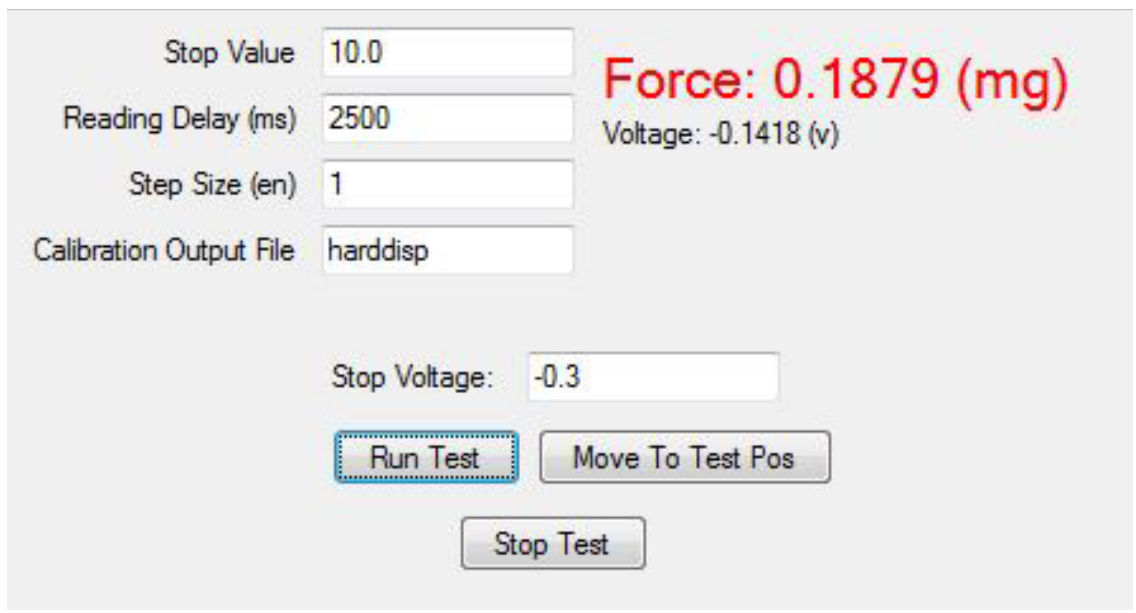

Figure 92: Configuration of reading delay and step size for testing. 


\section{SYSTEM SET UP}

The MFDS is comprised of various components all of which serve specific purposes (Figure 93).

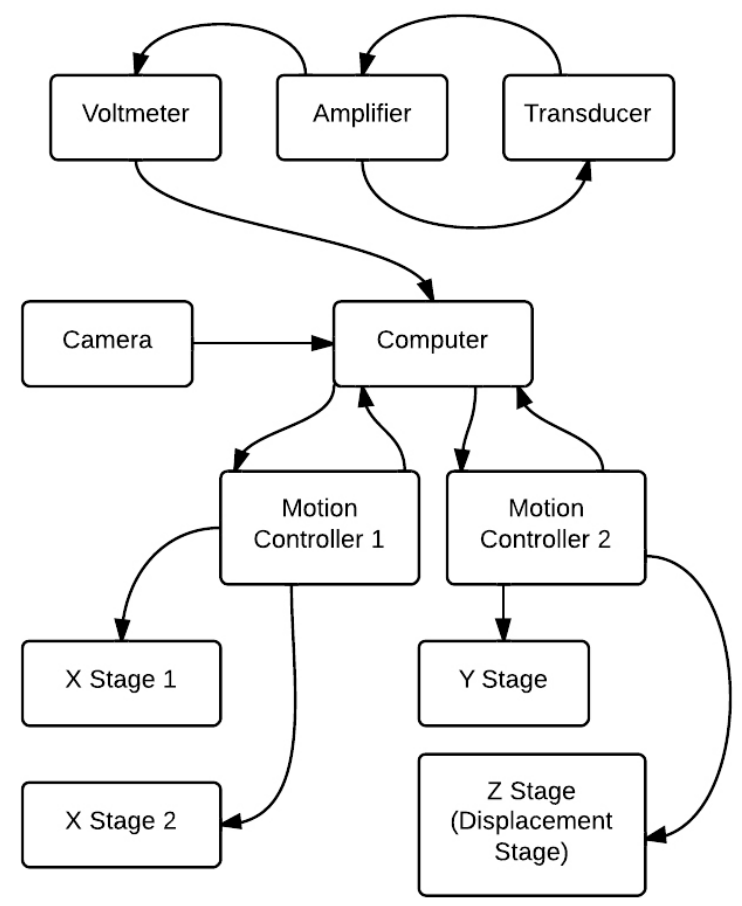

Figure 93: Block diagram of MFDS.

\section{MOTION CONTROLLERS}

The motion controllers are responsible for translating commands from the computer into electrical signals for the linear stages. There are 2 motion controllers for the 4 linear stages. The top motion controller (1) is responsible for controlling the y axis (1.1) as well as the $z$ axis (1.2). The bottom motion controller (2) is responsible for controlling both the top $x$ axis (2.1) and the bottom $x$ axis (2.2)(Figure 94). 


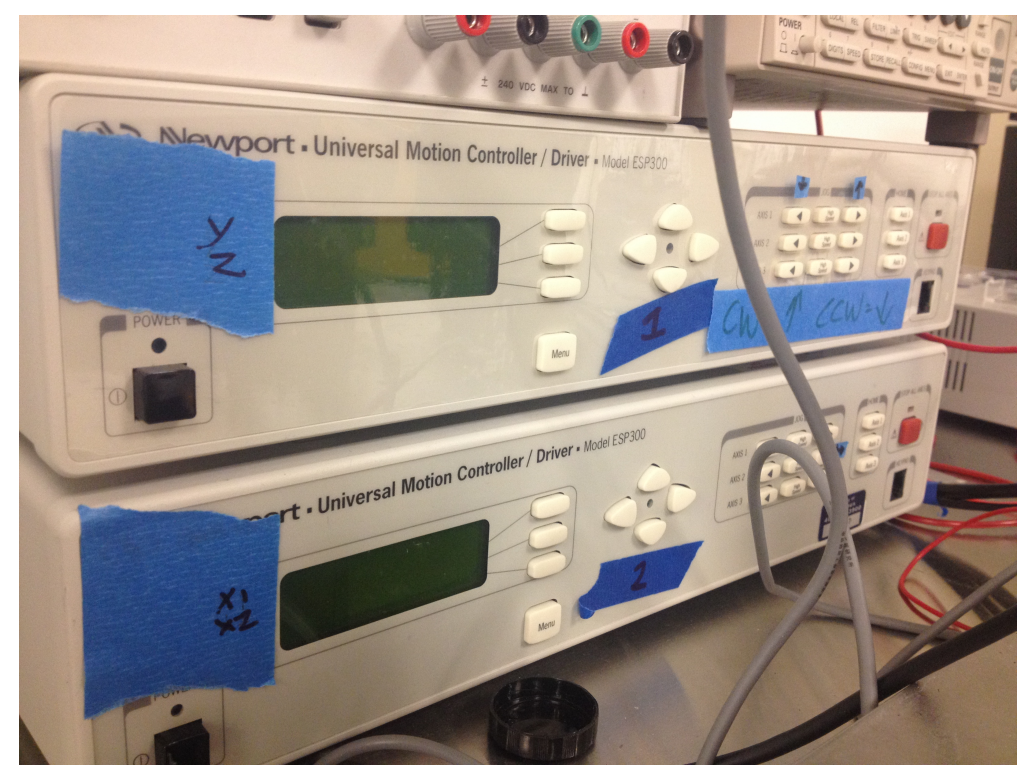

Figure 94: ESP 300 Motion Controllers, with (1) on the top and (2) on the bottom..

The back of the motion controllers has the RS-232 input, the axis input and the power cord. For motion controller 1 , the $y$ axis is plugged in on the right and the $z$ axis is plugged in on the left. For motion controller 2, the top $\mathrm{x}$ axis is plugged in on the right and the bottom $x$ axis is plugged in on the left (Figure 95).

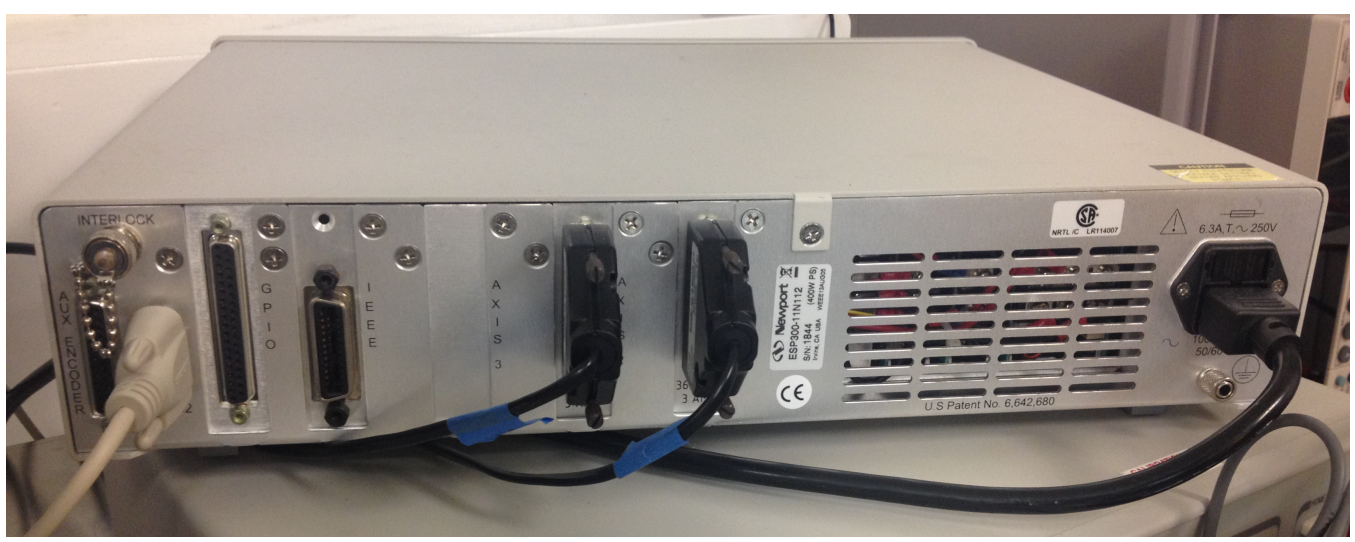

Figure 95: Motion controller set up. From left to right: RS-232 input, Axis 2 input, Axis 1 input, Power cord. 
The RS-232 output from the motion controllers is fed into a RS-232 to USB adapter, then plugged into the back of the computer. It is important to note the position of the USB cables due to the fact that the software addresses each component based on the COM port it is utilizing. If the components are not plugged into the same USB ports, then the system will incorrectly address each component, leading to software errors. The location of the USB ports for the motion controllers is shown in Figure 96.

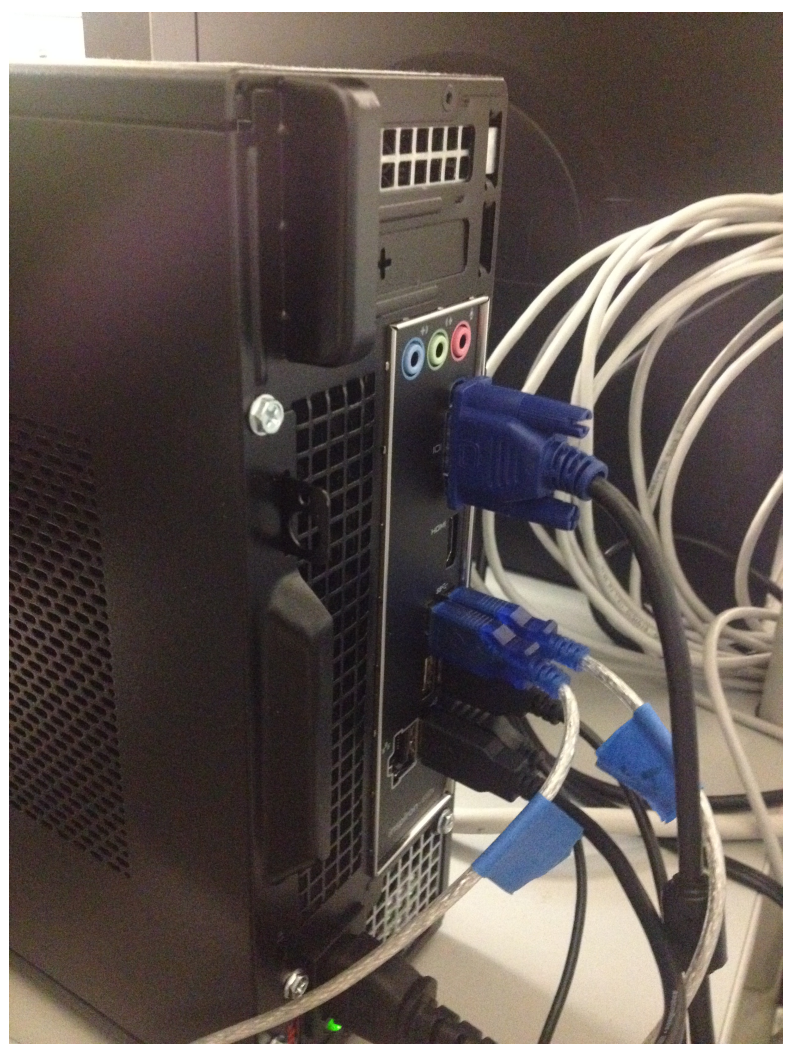

Figure 96: RS-232 to USB converters plugged into the computer. The blue USB cable closest to the camera is for motion controller 1 , while the blue USB cable further from the camera is for motion controller 2 .

\section{LINEAR STAGES}

There are 4 linear stages in the system. Two $x$ axis stages, one $y$ axis stage and one $z$ axis stage. The motion controllers are fastened to the optical bench with screws, and can be adjusted to fit individual needs. If the emergency stop is activated on the 
motion controllers due to stage movement, this indicates that the stages are experiencing too much motion, and should be moved closer to one another.

CAMERA

The camera used is a The Imaging Source DMK-72AUC02 camera with a M0814-MP2 lens. The aperture and focus adjustments can be found on the side of the lens barrel. In addition the camera can be moved on the mounting bracket to magnify images if necessary. The camera communicates via USB with the computer, although is insensitive to the COM port unlike the motion controllers and voltmeter.

\section{TRANSDUCER}

The transducer is the most essential component of the system. The transducer is mounted on an aluminum bracket to the $z$ axis stage which allows for positive (down) and negative (up) movements of the transducer assembly. The tip of the transducer is glued to the interior of a vented vacuum screw, then secured to the transducer cantilever arm. The output from the transducer is the input for the amplifier (Figure 98)

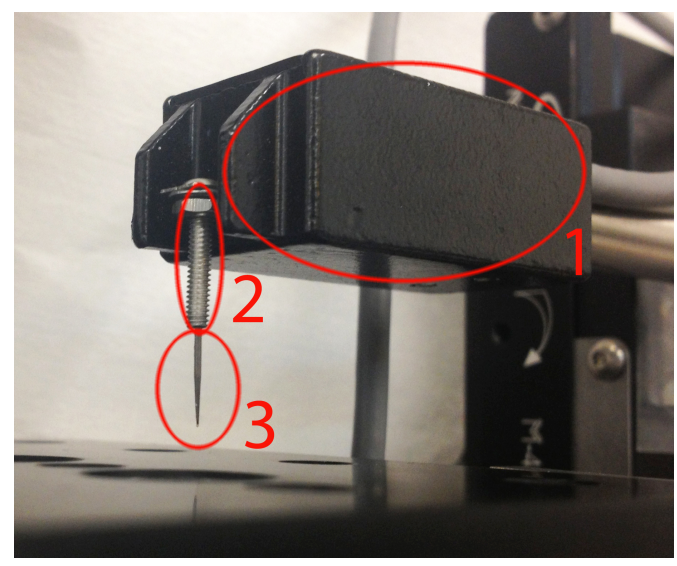

Figure 97: Transducer assembly. 1) Transducer body 2) vented vacuum screw 3) transducer tip. 


\section{AMPLIFIER}

The amplifier used is a World Precision Instruments TBM4M. The amplifier provides voltage to the transducer, as well as receives the output signal, filters some of the noise, and amplifies the signal. The amplifier is set up at $1000 \mathrm{X}$ amplification, with the 'Diff' switch activated indicating differential noise filtering (Figure 98).

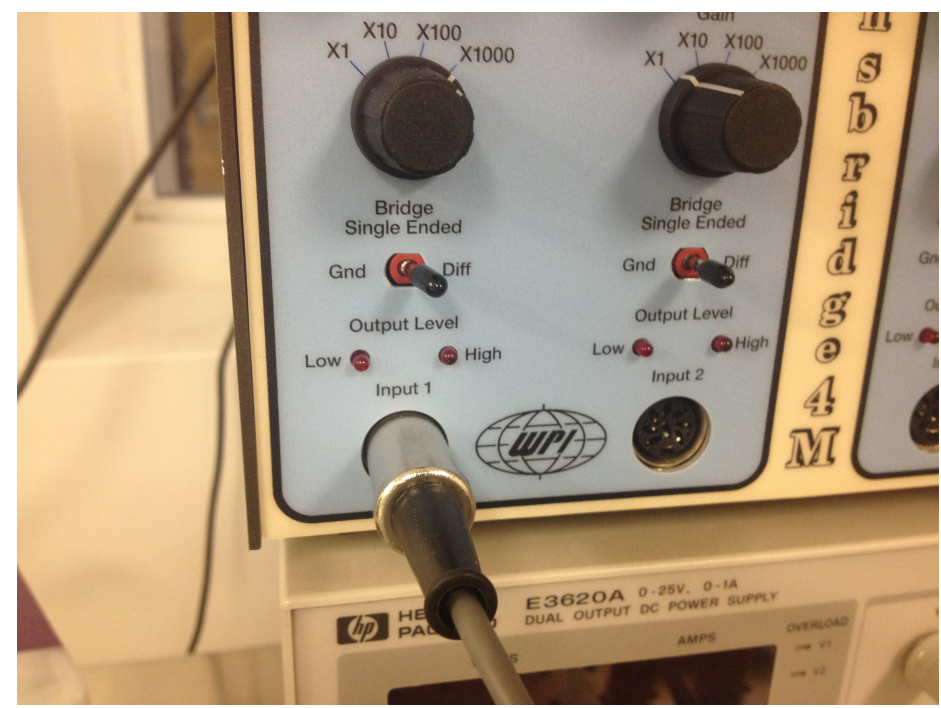

Figure 98:Front of the World Precision Instruments TBM4M amplifier, note that the Bridge Single Ended is switched to 'Diff' to remove noise from the output. The plug in the front of the amplifier is the output from the transducer.

The back of the amplifier shows the BMC output cable that runs to the voltmeter (Figure 99). 


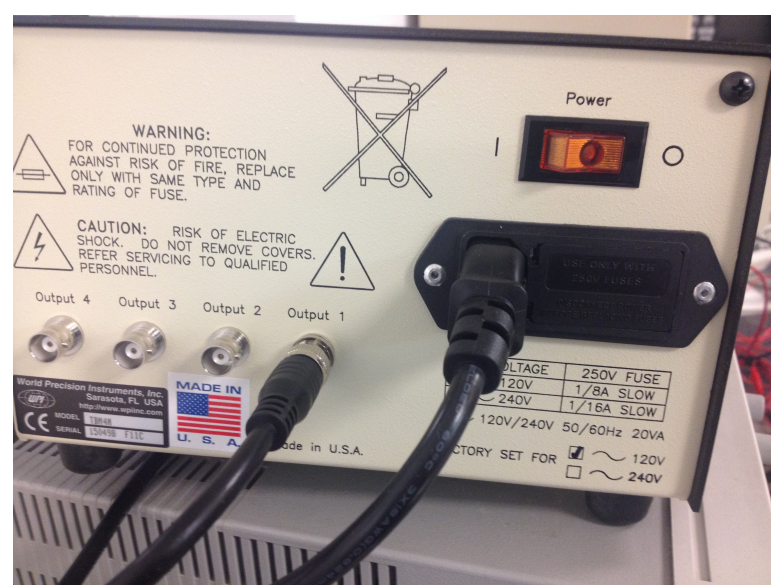

Figure 99: Back of World Precision Instruments TBM4M amplifier. The power switch and power cable are shown. The BMC cable plugged into Output 1 will go to the voltmeter.

\section{VOLTMETER}

The voltmeter used was a Keithley 2400 SourceMeter. The software is set up such that a reading will be taken from the voltmeter at specified intervals (Figure 92). The BMC cable coming from the Output 1 of the amplifier (Figure 99) is converted into two banana cables (Figure 100), which then feed into the Input/Output ports on the front of the voltmeter (Figure 101).

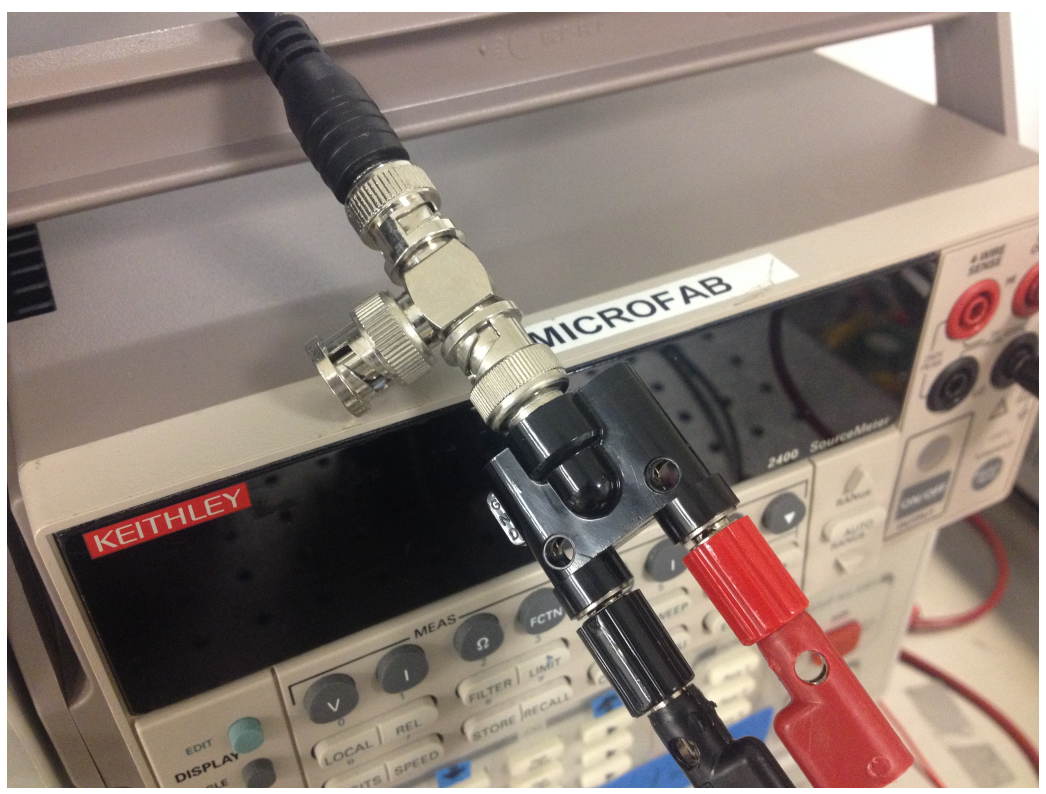

Figure 100: Converting the BMC cable to banana cables. 


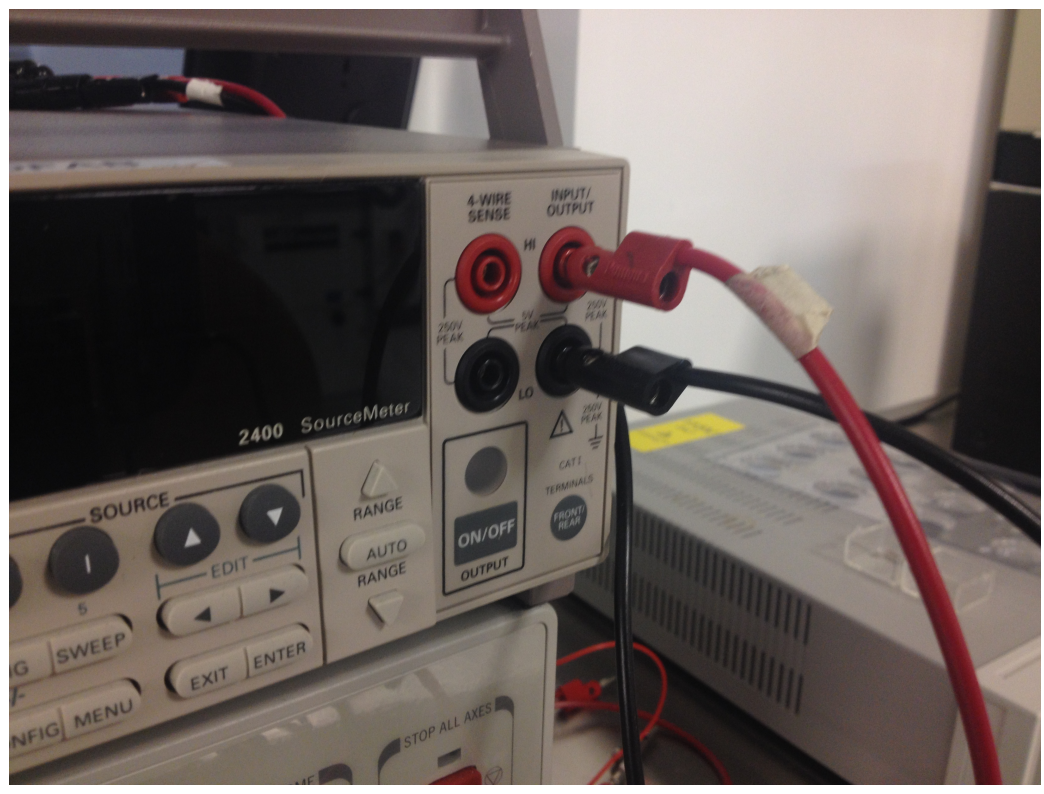

Figure 101: Cables from amplifier plug into the front of the voltmeter.

The back of the voltmeter is used only for the power cable and RS-232 output to the computer (Figure 102).

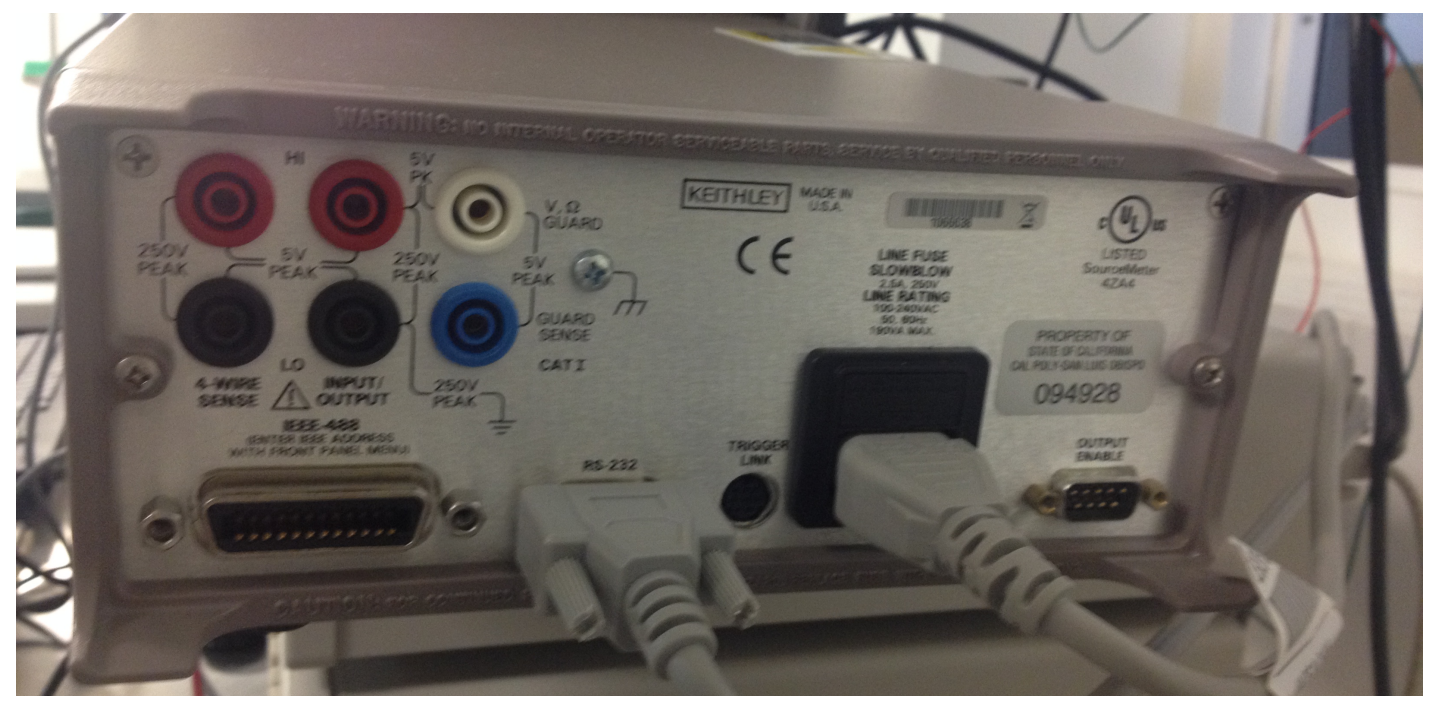

Figure 102: Back of voltmeter, with RS-232 output (left) and power cable (right).

When setting up the Keithley 2400 , it is important to make sure the settings for communication are correct. With the voltmeter turned on, select MENU, then COMMUNICATION, then RS-232. Ensure that the RS-232 settings are as follows: 
BAUD- 19200

BITS- 8

PARITY- NONE

TERMINATOR- <CR + LF>

FLOW CONTROL- NONE

As with the motion controllers, it is critical to make sure the voltmeter is plugged into the same USB port during each operation. The proper USB port for the voltmeter is shown in Figure 103.

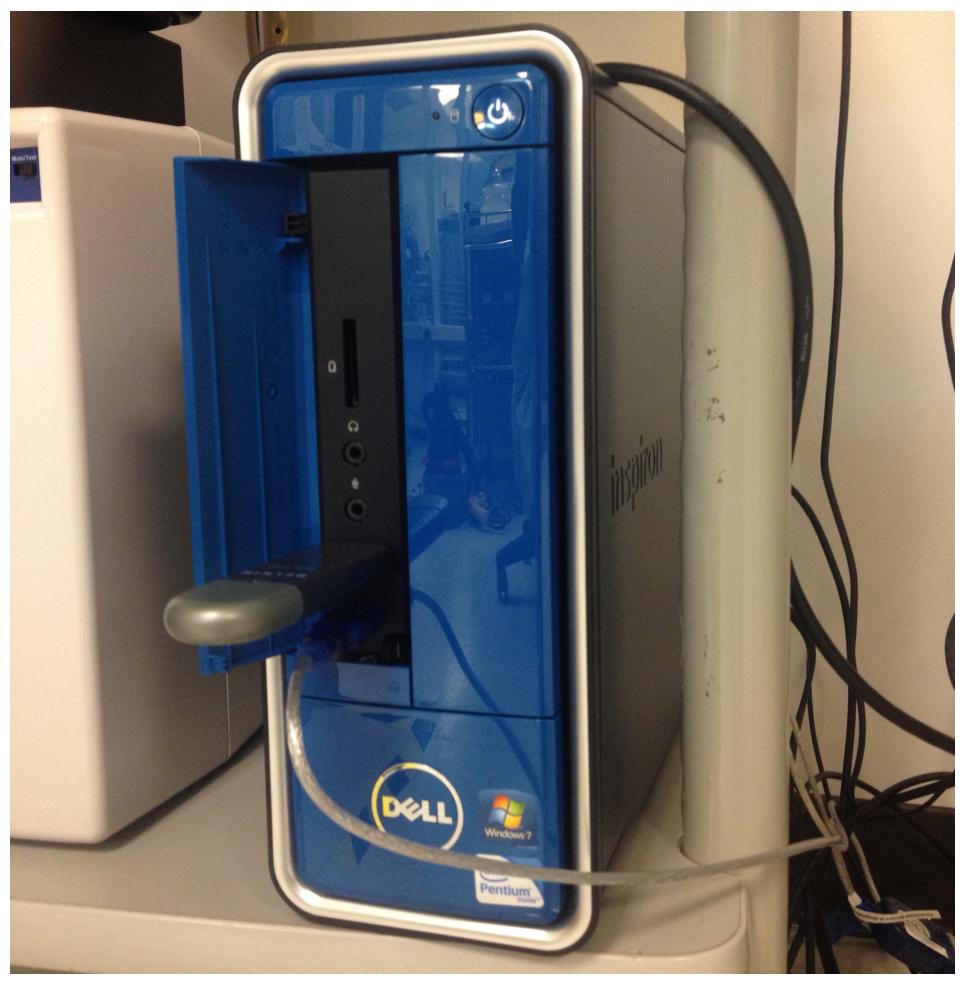

Figure 103: Location of Keithley 2400 input to computer. The Silver cable is the voltmeter input on the front of the computer. 
TESTING

- To begin testing, first turn on the motion controllers (Figure 104 and Figure 105), voltmeter (Figure 106), and amplifier (Figure 107). Be sure to activate each motion controller stage by pressing the white button next to each stage. Turn on all equipment 2 hours prior to testing to let the components warm up. Next adjust the amplifier until both the high and low LEDs are lit (this indicates that the amplifier has been zeroed).

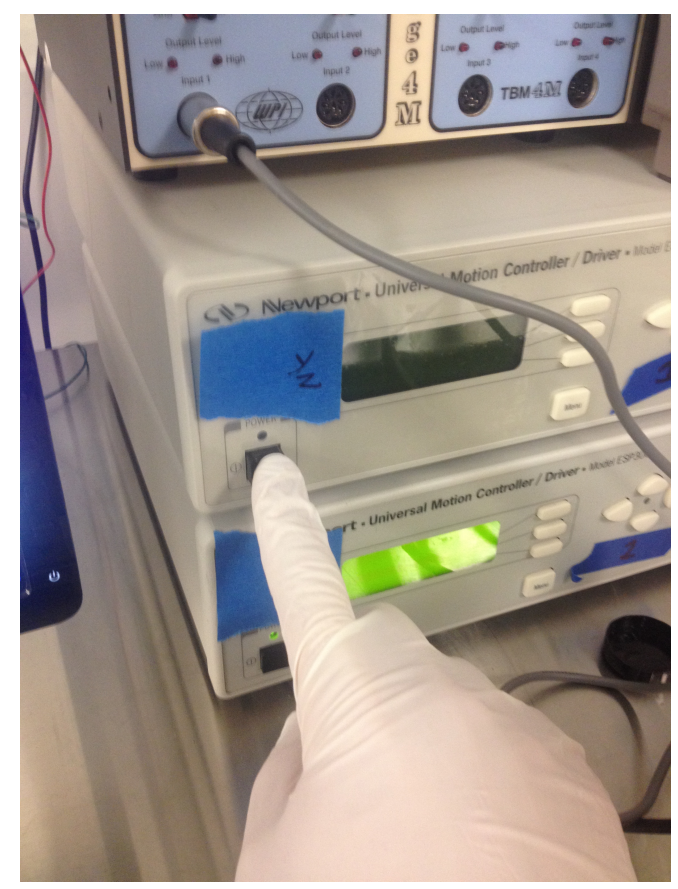

Figure 104: Turning on motion controller 1. 


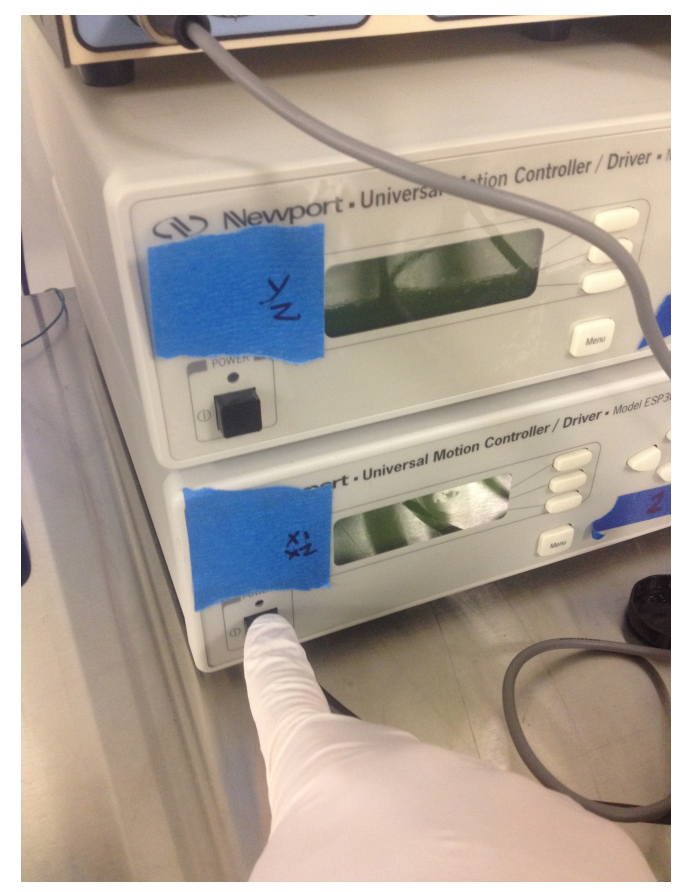

Figure 105: Turning on motion controller 2.

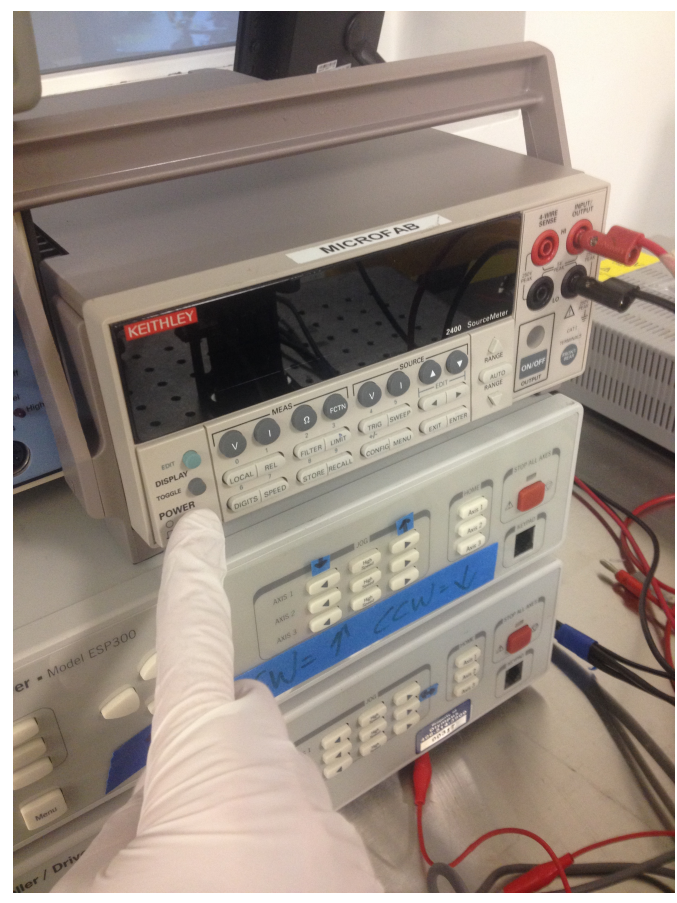

Figure 106: Turning on the Keithley 2400 voltmeter 


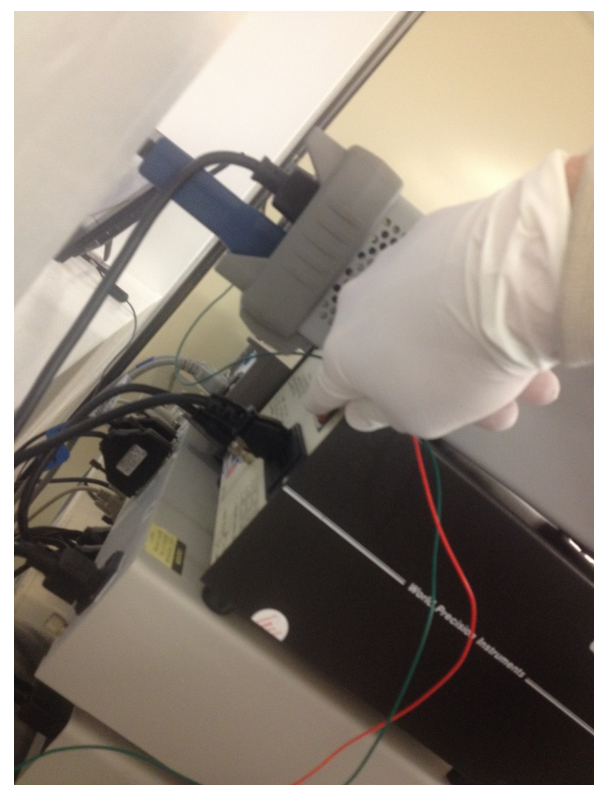

Figure 107: Turning on the amplifier, the switch is top left as viewed from the front of the amplifier.

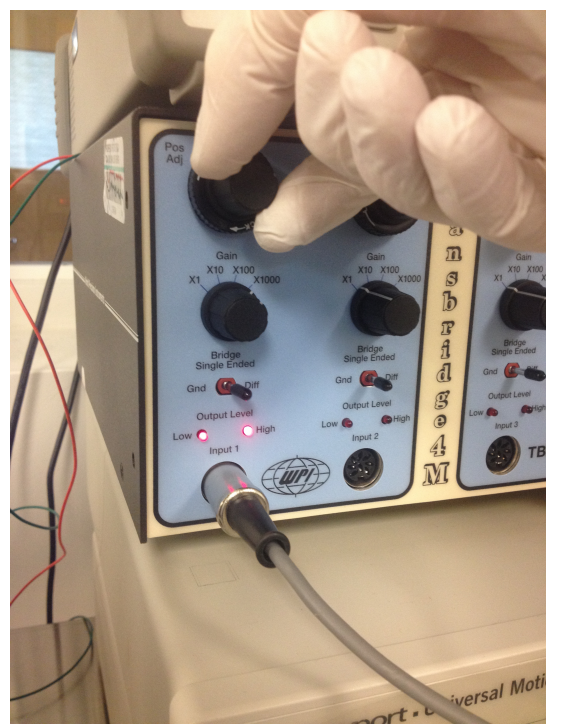

Figure 108: Adjusting amplifier to achieve OV output as indicated by both the low and high LEDs lit. 
- Open the VS Express for Desktop application found in the Microsoft Visual Studio 2012 Express folder.

- Open the MFDMInterface project found at:

C:IUsers\microfab\Documents|GitHublMicroforceDisplacementMachinelMFDMInterface.

- To begin the program select debug>Start Debugging

- The program will begin and ask to select a Video Capture Device. From the drop down menu select 'DMK 72AUC02' (Figure 109).

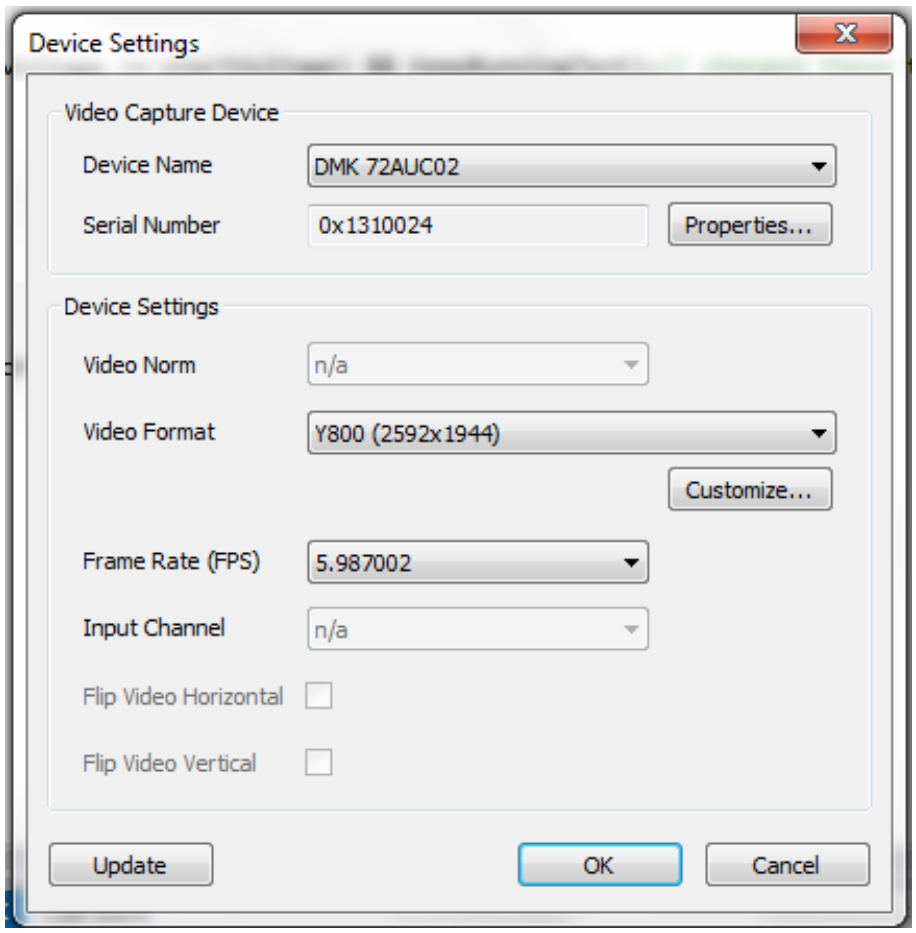

Figure 109: Video Capture Device selection, with DMK 72AUC02 selected as the device name.

-With the MFDM Controller open, a test can now be initiated. 
- Place the sample to be tested on the top $x$ axis stage. Align the sample using the up, down, left, and right buttons as well as the top step size slider to control the size of each movement.

- Once the proper alignment has been achieved, select the move to test position button, which will bring the sample under the transducer (be sure the transducer is not low enough to be hit by the sample).

- Using the Vertical Step Size slider, select a value appropriate to bring the transducer close to the surface of the sample ( 2000en). As the transducer tip approaches the sample, when the gap between is too small to safely move the transducer down anymore, change the Vertical Step Size to $\sim 200$ en and lower one step at a time.

- Watch the LEDs on the front of the amplifier (which are currently both lit, indicating 0V), as the transducer contacts the sample, the low LED will no longer remain lit, while the high LED will remain lit, indicating that a positive voltage has been registered from sample contact.

-With the high LED lit, move off the sample until both LEDs are again lit. Now the transducer is close to contacting the sample, but will contact the sample once the testing begins.

- All relevant testing parameters can now be entered. To choose the output folder, find the line under the TestingUtility.cs tab that contains a file path name, manipulate the file path to the desired folder. The reading delay can now be set. Typical tests are run at $1000 \mathrm{~ms}$ reading delay with a step size of $1 \mathrm{en}$ (to give maximum resolution). If the repetitive test radio button is selected, the number of test repetitions can be entered. Finally the Stop Voltage needs to be entered (remember the transfer function is $\mathbf{5 5 . 1 0 2}$ mg per V). 
- The test is performed by selecting the Run Test button.

-The test will stop once the Stop Voltage is reached (or the Stop Test button is pressed).

- Once the test has stopped, the transducer can be moved off the sample, and the backtrack button will return the sample to its initial position under the camera cross hairs.

-When finished testing, Close the MFDM Controller, then close Microsoft Visual Studio Express 2012. Turn off the amplifier, voltmeter, and motion controllers.

\section{AND Y AXIS CALIBRATION}

In order for the user to utilize the cross hairs of the camera view to align samples, the offset from the center of the camera to the tip of the transducer must be known by the system. To calibrate the $X$ and $Y$ stages, a small amount of silicone grease is placed on the transducer tip. A glass microscope slide is then placed onto the testing specimen area. The tip of the transducer is brought into contact with the glass slide, and then lifted off. The "XY Calibrate" button is then selected. At this point the software used the assumed offset to bring the silicone grease "dot" close to the center of the camera view. The $X$ and $Y$ stages are then used to position the dot under the crosshairs of the camera. Once satisfied with the position, the "Set Offset" button is selected. Through the course of calibration, the software tracks the movements made by the user and added or subtracted the displacement values from the assumed offset to produce the actual offset. Once the calibration is complete, a sample can be placed into the MFDS and aligned using the crosshairs. The "Move To Test Pos" button can then be pressed to move the sample from the camera crosshairs to the transducer tip. Repeating this process multiple times insures that the $\mathrm{x}$ and $\mathrm{y}$ stages return to the same position each 
time during calibration. If the transducer tip is replaced, or the system is dismantled and reassembled, the $X \& Y$ axis calibration must be performed to insure correct alignment.

\section{DATA ANALYSIS}

The data analysis is used to convert the raw output of the voltmeter into useable data. With the output data, only the second column is important, which gives the voltmeter reading at intervals set by the reading delay. With the knowledge that the $z$ axis is moving by the step size during each reading delay, the difference in displacement between each voltage reading is the step size (for most tests equal to 1 en). Plotting the initial data shows the noise before the transducer has contacted the sample, the dip from Vander Waals forces, then the increase in force from the test itself (Figure 110).

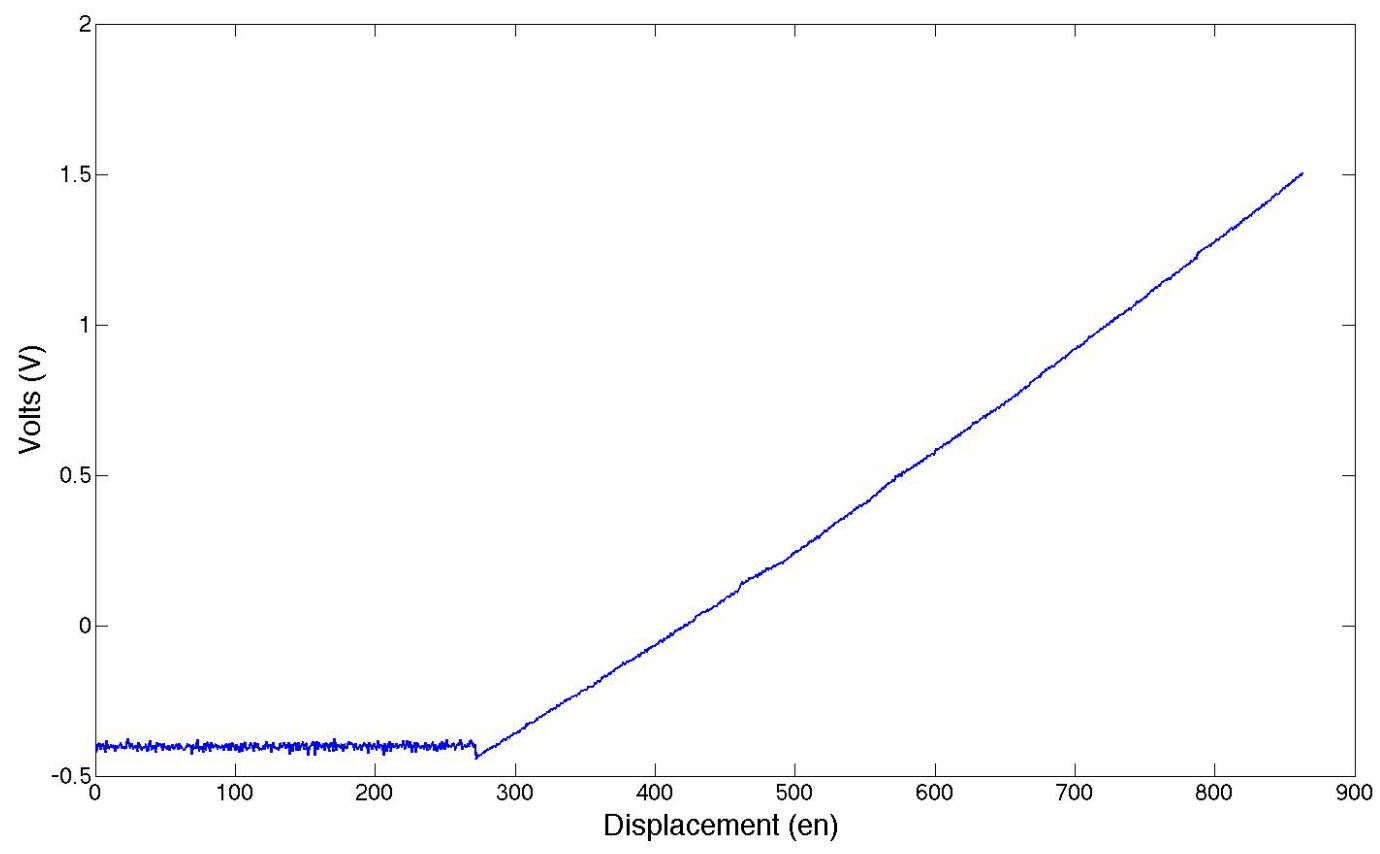

Figure 110: Initial plotting of output data from testing.

The following is a simplified version of Matlab code used for processing the output data:

oimport the data file into matlab using whichever method you 
prefer.

oWith the data portion imported as the variable data with displacement oinformation in column 1 and voltage information in column 2

okeep only the voltage data from the test

$\operatorname{data}=\operatorname{data}(:, 2)$

\&Look at the initial data (figure 20)

plot (data)

ofind the point at which the test starts (in this case the plot oindicates the data begins at data point 272)

$\operatorname{data}=\operatorname{data}(272:$ end $)$

otranslate the data so it begins at 0 volts

data $=\operatorname{data}-\operatorname{data}(1)$

○Create a displacement vector

$\mathrm{x}=1:$ length $($ data $)$

$\mathrm{x}=\mathrm{x}^{\prime}$

\%create a vector that represents the systemic displacement

$(0.0056$ in units of $\mathrm{V} / \mathrm{en})$ is the 


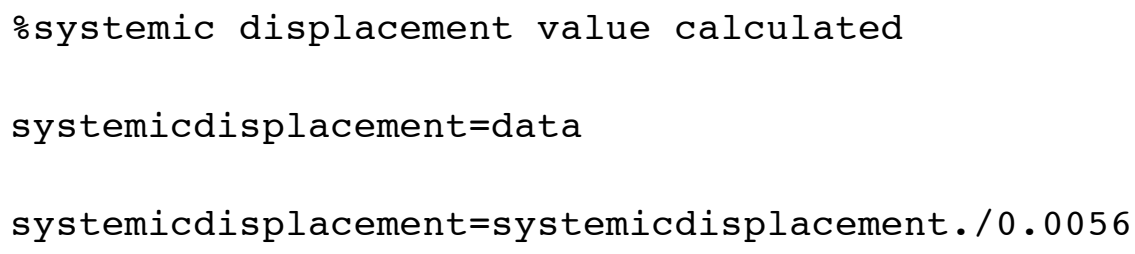

After the transformations from the Matlab code, the plot generated is in units of force (mg) and displacement (um) 


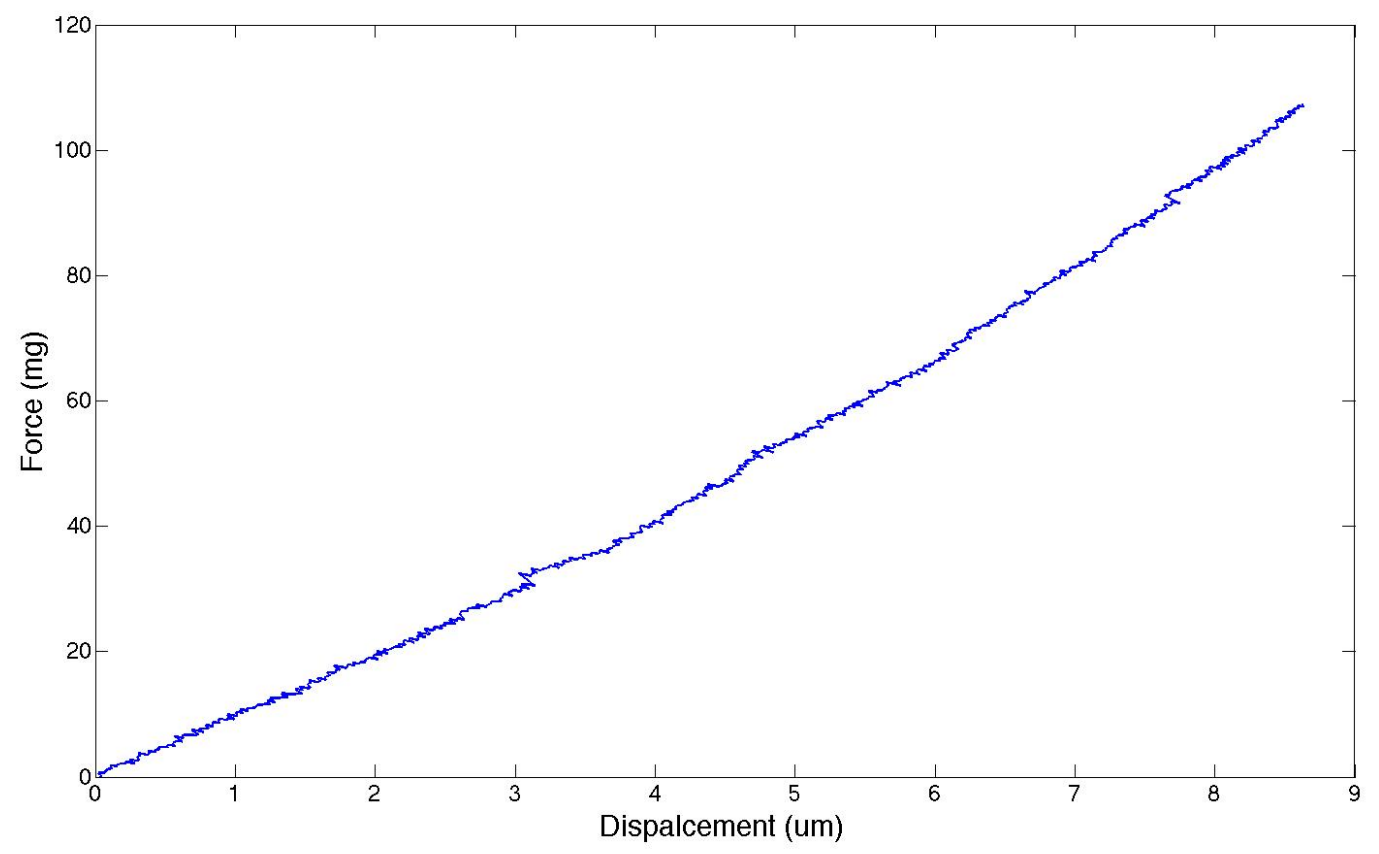

Figure 111: Data after transformations to create the proper units. 